Alma Mater Studiorum - University of Bologna

$\mathrm{PhD}$ in Control System Engineering and Operational Research Cycle XIV

Settore concorsuale di afferenza: 01/A6 - RICERCA OPERATIVA

Settore scientifico disciplinare: MAT/09 - RICERCA OPERATIVA

\title{
Exact Algorithms for Different Classes of Vehicle Routing Problems
}

\author{
Roberto Roberti \\ Coordinator \\ Advisors \\ Prof. Alberto Caprara \\ Prof. Aristide Mingozzi \\ Prof. Paolo Toth
}

Final Exam 2012 



\section{Contents}

1 Introduction 1

1.1 On Logistics Problems . . . . . . . . . . . . . . . . . . . . . . 1

1.2 Reasons for Research . . . . . . . . . . . . . . . . . . . 4

1.3 Contents and Contributions of the Thesis . . . . . . . . . 5

1.3.1 Asymmetric Traveling Salesman Problem . . . . . . . . . . . . 5

1.3.2 Traveling Salesman Problem with Time Windows . . . . . . . . . . 6

1.3.3 Vehicle Routing Problem with Time Windows . . . . . . . . . . 7

1.3.4 Multi-Trip Vehicle Routing Problem . . . . . . . . . . . . . 8

1.3.5 Two-Echelon Capacitated Vehicle Routing Problem . . . . . . . 9

2 Asymmetric Traveling Salesman Problem $\quad 11$

2.1 Introduction . . . . . . . . . . . . . . . 11

2.2 The Dantzig-Fulkerson-Johnson Formulation and its Relaxations . . . . 12

2.3 Review of Polynomial Formulations . . . . . . . . . . . . . . . . . . 14

2.4 Exact Algorithms . . . . . . . . . . . . . . . . . . . . . . 19

2.5 Transformation of ATSP Instances into STSP Instances . . . . . . . . . . . 22

2.6 Computational Results . . . . . . . . . . . . . . . . . 22

3 Traveling Salesman Problem with Time Windows 29

3.1 Introduction . . . . . . . . . . . . . . . . . . . . . . . . . . . . . . . 29

3.2 Literature Review . . . . . . . . . . . . . . . . . . . . . . . . . . . . . . . 30

3.3 An Exact Algorithm for the TSPTW . . . . . . . . . . . . . . 31

3.4 Relaxations of the TSPTW . . . . . . . . . . . . . . . . . . . . . . . 33

3.4.1 The $t$-Tour Relaxation . . . . . . . . . . . . . . . . . . 33

3.4 .2 The $n g$-Tour Relaxation . . . . . . . . . . . . . . . . . . . 34

3.4.3 The $n g L$-Tour Relaxation . . . . . . . . . . . . . . . . . . . . . . . . . . . . . . . . .

3.5 A Valid Lower Bound $L B$ on the TSPTW . . . . . . . . . . . . . . . 38

3.5.1 Initializing the Master Problem . . . . . . . . . . . . . . . . . 39

3.5.2 Solving the Master Problem . . . . . . . . . . . . . . . . . . . . . . . 40

3.5.3 Solving the Pricing Problem . . . . . . . . . . . . . . . . . 41

3.6 Computing Bounding Functions $b(S, t, i) \ldots \ldots \ldots \ldots$

3.7 Computational Results . . . . . . . . . . . . . . . . . . . . . 44 44

3.7 .1 Parameter Setting . . . . . . . . . . . . . . . . . . . . . . . . . . . . . . . 45

3.7 .2 Ascheuer Instances . . . . . . . . . . . . . . . . . . . . . . . . . . . . . . . . . . 47

3.7.3 Pesant Instances . . . . . . . . . . . . . . . . . . . . . . . 49

3.7 .4 Potvin Instances . . . . . . . . . . . . . . . . . . . . . . 49

3.7 .5 Gendreau Instances . . . . . . . . . . . . . . . . . . . 49 
3.7 .6 Olhmann Instances . . . . . . . . . . . . . . . . . . . . . . 51

3.7.7 More Details on the Computational Results . . . . . . . . . . . 53

3.8 Conclusions . . . . . . . . . . . . . . . . . 55

4 Vehicle Routing Problem with Time Windows $\quad \mathbf{5 7}$

4.1 Introduction . . . . . . . . . . . . . . . . . . . . 57

4.2 Literature Review . . . . . . . . . . . . . . . . . . . 58

4.3 Mathematical Formulation and its Relaxations . . . . . . . . . . . 60

4.3.1 Set Partitioning Formulation . . . . . . . . . . . . . . . . 61

4.3 .2 Relaxation $L P \ldots \ldots \ldots$. . . . . . . . . . . . . 6 . . . . . . . 61

$4.3 .3 n g$-Route Relaxation for the CVRP . . . . . . . . . . . . 62

4.4 An Exact Algorithm . . . . . . . . . . . . . . . . . . . . . 63 63

4.5 Bounding Procedures $H^{1}, H^{2}$ and $H^{3} \ldots \ldots \ldots$. . . . . . . 64

4.5.1 Algorithm CCG . . . . . . . . . . . . . . . 65

4.5.2 Bounding Procedure $H^{1} \ldots \ldots \ldots \ldots$. . . . . . . . . . . . . . . . . . . 68

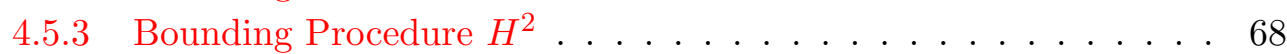

4.5.4 Bounding Procedure $H^{3} \ldots \ldots \ldots \ldots$. . . . . . . . . . . . . . . . . . . . . . 69

4.5.5 Procedure GenR . . . . . . . . . . . . . . . . . . 69

4.5.5.1 Procedure GenP. . . . . . . . . . . . . . . . . 70

4.5.5.2 Procedure Combine. . . . . . . . . . . . . . 73

4.5.5.3 GenR for the CVRP. . . . . . . . . . . . . . . . . . . . . . . . . . . . . .

4.6 Column-and-Cut Generation Procedure $H^{4} \ldots \ldots \ldots$. . . . . . . . . . . . . . . . . . . . . . . .

4.6 .1 Procedure GenR4 . . . . . . . . . . . . . . . . . . . . . . . . . . . . . . . 74

4.6 .2 Procedure GenP4 . . . . . . . . . . . . . . . . . . . . . . . . 74

4.6 .3 Procedure Combine4 . . . . . . . . . . . . . . . . 76

4.6.4 Procedure GenR4 for the CVRP . . . . . . . . . . . . 76

4.7 Procedure GenRF for Generating Route Set $\widetilde{\mathscr{R}} \ldots \ldots$. . . . . . . . . . 76

4.7 .1 Procedure GenPF . . . . . . . . . . . . . . . . . . . . . . . . . . . . . . . . . . . .

4.7 .2 Procedure CombineF . . . . . . . . . . . . . . . . . . . . . . . . . . 78

4.8 Computational Results . . . . . . . . . . . . . . . . . 78

4.8.1 Computational Results on the VRPTW . . . . . . . . . . . 78

4.8.2 Computational Results on the CVRP . . . . . . . . . . . . . . 81

4.9 Conclusions . . . . . . . . . . . . . . . . . . . 82

5 Multi-Trip Vehicle Routing Problem 95

5.1 Introduction . . . . . . . . . . . . . . . . . . 95

5.2 Literature Review . . . . . . . . . . . . . . . . . . . . . . . . . . . . . . . . . . . .

5.3 Mathematical Formulations and Relaxations . . . . . . . . . . . . 97

5.3 .1 Formulation $F 1 \ldots \ldots \ldots \ldots \ldots$. . . . . . . . . . . . . . . . . . . . . . . . . . .

5.3 .2 Formulation $F 2 \ldots \ldots \ldots \ldots \ldots \ldots$. . . . . . . . . . . . . . . . . . . 98

5.3.3 Comparing Relaxations $L F 1$ and $L F 2 \ldots \ldots \ldots \ldots$

5.3 .4 Relaxation $R F 1$. . . . . . . . . . . . . . . . . . . . 100

5.4 Improving Relaxations $L R F 1$ and $L F 2$ and Outline of the Exact Method101

5.4 Relaxation $\overline{L R F 1} \ldots \ldots$. . . . . . . . . . . . . . . . . . . . 101

5.4 .2 Relaxation $\overline{L F 2} \ldots \ldots \ldots$. . . . . . . . . . . . . . 105

5.4.3 Using Multiple Dual Solutions to Improve Procedures $H^{2}, H^{3}$

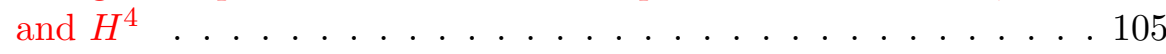


5.5 Bounding Procedures $H^{1}, H^{2}$, and $H^{3}$ Based on Relaxation $\overline{L R F 1} \ldots 107$

5.5.1 Algorithm CCG . . . . . . . . . . . . . . . . . 107

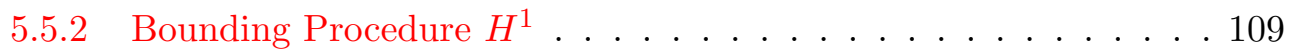

5.5.3 Route Generation Algorithm GenR . . . . . . . . . . . . . . 109

5.5.3.1 Phase 1 of GenR. . . . . . . . . . . . . . . . . . . . . . . . . . . . . . .

5.5 .3 .2 Phase 2 of GenR. . . . . . . . . . . . . . . . . . . . . . . . . . . . . . . .

5.5 .4 Bounding Procedure $H^{2} \ldots \ldots \ldots$. . . . . . . . . . . . . . . . . . . . . . . . . . .

5.5.5 Generating the Path Set $\mathscr{P}^{2}$ and the Route Set $\mathscr{R}^{2} \ldots \ldots . . .111$

5.5 .6 Bounding Procedure $H^{3} \ldots \ldots \ldots$. . . . . . . . . . 112

5.5.7 Generating the Route Set $\mathscr{R}^{3}$ and Solving $F 1 \ldots \ldots . . . . . . .113$

5.6 Bounding Procedure $H^{4}$ Based on Relaxation $\overline{L F 2} \ldots \ldots$. . . . . . . . 113

5.7 Algorithm GenSched to Generate Vehicle Schedules . . . . . . . . . . . . 114

5.8 Description of the Exact Method to Solve the MTVRP . . . . . . . . . 115

5.9 Computational Results . . . . . . . . . . . . . . . . . . 116

5.10 Conclusions . . . . . . . . . . . . . . . . . . . . . 120

6 Two-Echelon Capacitated Vehicle Routing Problem 121

6.1 Introduction . . . . . . . . . . . . . . . . . . . . . . . . . 121

6.2 Literature Review . . . . . . . . . . . . . . . . . . . . . . . 122

6.3 Problem Description and Mathematical Formulation . . . . . . . . . 123

6.4 Relaxations of Formulation $F \ldots \ldots$. . . . . . . . . . . . . . . . . . . . . . .

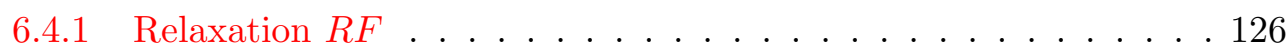

6.4.2 Relaxation $\overline{R F} \ldots \ldots \ldots \ldots$. . . . . . . . . . . . 131

6.5 Lower bound $L D 1$ and bounding procedure $D P^{1} \ldots \ldots$. . . . . . . . . . . . . . . . . . . . .

6.5.1 Description of Procedure $D P^{1} \ldots \ldots \ldots \ldots$. . . . . . . 135

6.5.2 Solving Problem $\overline{R F} \ldots \ldots$. . . . . . . . . . . . 136

6.5.3 Computing a subgradient . . . . . . . . . . . . . 136

6.5.4 Generating the $n g$-route set $\mathscr{N}_{k}$ for a given satellite $k \ldots 137$

6.5.5 A Lagrangean heuristic . . . . . . . . . . . . . . . . . . . . . . 138

6.6 An exact method for solving the 2E-CVRP . . . . . . . . . . . . 140

6.6.1 Generating the Set of Configurations $\mathscr{P} \ldots \ldots \ldots$. . . . . . . 141

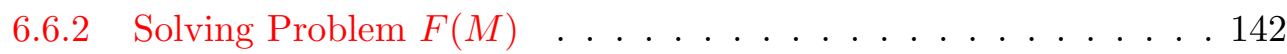

6.6.2.1 Phase 1: computing lower bound $L D 1(M) \ldots \ldots . . .142$

6.6.2.2 Phase 2: solving $\bar{F}(M) \ldots \ldots \ldots$. . . . . . . . . . . . 142

6.6.2.3 Phase 3: solving $F(M)$ to optimality . . . . . . . 143

6.6.3 Description of the Exact Method . . . . . . . . . . . . . . . . 143

6.7 Computational results . . . . . . . . . . . . . . . . . . . 145

6.8 Conclusions . . . . . . . . . . . . . . . . . . . . . 148

$\begin{array}{ll}\text { Bibliography } & 155\end{array}$ 



\section{Keywords}

- Vehicle Routing Problems

- Traveling Salesman Problems

- Time Windows

- Exact Algorithms

- Column Generation

- Dynamic Programming

- State-Space Relaxation

- Dual-Ascent Heuristics 

Ai miei Genitori 



\section{Acknowledgements}

I want to express my deeply-felt thanks to my thesis advisors, Professors Aristide Mingozzi and Paolo Toth, for their warm encouragement, thoughtful guidance, and insightful criticism.

I also want to express my gratitude to Professor Roberto Baldacci for his continuous support and sound advice.

Lastly, I wish to thank Dr. Enrico Bartolini, Dr. Valentina Cacchiani, Professor Alberto Caprara, and Professor Roberto Wolfler Calvo. 



\section{Chapter 1}

\section{Introduction}

\subsection{On Logistics Problems}

Logistics is defined by Eilon and Christofides [1971] as "the provision of goods and services from a supply point to a demand point". Despite such a short definition, logistics systems span a large spectrum of decision-making activities. Examples of such activities (see Simchi-Levi et al. [2005]) are as follows:

- Truck Routing. A truck must leave a warehouse to deliver products to a set of retailers. The order in which the retailers are visited will determine how long the delivery will take and at what time the vehicle can return to the warehouse. Thus, the vehicle must follow an efficient route.

- Vehicle Fleet Management. A warehouse must supply products to a set of retailers using a fleet of vehicles of limited capacity. A dispatcher is in charge of assigning loads to vehicles and determining vehicle routes. Firstly, the dispatcher must decide how to partition the retailers into groups that can be feasibly served by a vehicle (i.e., whose total loads fit in a vehicle). Secondly, the dispatcher must decide what sequence to use so as to minimize the total traveled distance of the vehicles. Therefore, the warehouse incurs a cost that depends on both the assignment of the retailers to the vehicles and the routes performed by the vehicles. Both aspects must be considered simultaneously.

- Network Configuration. Several plants produce products to serve a set of retailers. Due, for example, to changes in demand patterns or in plant production or in supply costs, the current set of facilities (i.e., plants and warehouses) is deemed to be inappropriate, and management wants to reorganize the distribution network. Therefore, the management must choose a set of facility locations and capacities, determine production levels for each product at each plant, and 
set transportation flows from plants to warehouses and from warehouses to retailers, in such a way that total production, inventory and transportation costs are minimized and service level requirements are met.

The previous three activities are examples of problems that have been intensively studied in the field of operations research in the last few decades.

The first problem, indicated as truck routing, is known as traveling salesman problem (TSP). The TSP is to find a routing of a salesman who starts from a home location, visits a prescribed set of cities, and returns to the original location, in such a way that the total distance traveled is minimum and each city is visited exactly once.

A formal definition of the TSP is the following. Let $G=(V, E)$ be a complete undirected graph, where $V$ is the vertex set and $E$ is the edge set. A cost is associated with each edge $e \in E$. Let $\mathscr{H}$ be the family of all Hamiltonian cycles (tours) in graph $G$, where each tour of the set $\mathscr{H}$ has a cost equal to the sum of the costs of the edges traversed. The TSP is to find a tour of the set $\mathscr{H}$ of minimum cost.

The first work on the TSP is commonly owed to Menger [1932]. Various papers dealing with the TSP were published in the years following, but Robinson [1949] was the first to use the name "traveling salesman problem". An intense study of the TSP started with the seminal paper by Dantzig et al. [1954]. Since then, numerous survey papers and books were published on the TSP and its variants (see Lawler et al. [1985], Reinelt [1994], Gutin and Punnen [2002], and Applegate et al. [2007]). Garey and Johnson [1979] showed that the TSP is $\mathcal{N} \mathcal{P}$-hard.

The fields of applications of TSP go beyond the route planning problem of a traveling salesman and spans over several areas from mathematics to computer science, from genetics to electronics. Possible applications of the problem are computer wiring (Lenstra and Rinnooy Kan [1975]), wallpaper cutting (Garfinkel [1977]), dartboard design (Eiselt and Laporte [1991]), crystallography (Bland and Shallcross [1989]), machine scheduling problems, cellular manufacturing, arc routing problems, frequency assignment problems, structuring of matrices, and many more (see Gutin and Punnen [2002] for an exhaustive coverage).

The second problem, referred to as vehicle fleet management, is an example of $c a$ pacitated vehicle routing problem (CVRP), which can be stated as follows. A set of customers, each with a known location and a known requirement for some commodity, must be supplied from a single depot by a fleet of capacitated vehicles. The costs for traveling between each couple of customers and between the depot and each customer are known. The problem is to design the vehicle routes such that each customer is served by a single vehicle, the vehicle capacity is not violated in any of the routes, and the total routing cost is minimized. 
The CVRP can be formally described as follows. Let $G=\left(V^{\prime}, E\right)$ be an undirected graph, where $V^{\prime}=\{0,1, \ldots, n\}$ is the vertex set and $E$ is the edge set. Vertices 1 to $n$ correspond to a set of customers, whereas vertex 0 corresponds to the depot. A nonnegative cost is associated with each edge $e \in E$ and represents the travel cost spent to travel between the two vertices linked by edge $e$. A set of $m$ identical vehicles, each with capacity $Q$, is available at the depot. A nonnegative demand is associated with each customer. The CVRP consists of finding a collection of $m$ simple cycles (each corresponding to a vehicle route) of minimum total cost, defined as the sum of the costs of the edges belonging to the cycles, such that each cycle visits the depot, each customer is visited by exactly one cycle, and the sum of the demands of the vertices visited by a cycle does not exceed the vehicle capacity, $Q$.

The CVRP was first introduced, as the truck dispatching problem, by Dantzig and Ramser [1959], who proposed the first mathematical formulation and algorithm approach for the solution of the problem. The first heuristic approach for the CVRP is owed to Clarke and Wright [1964], who proposed an effective greedy heuristic. After these two seminal papers, a lot of effort has been made to model and solve the CVRP and tens of its variants. A number of books dealing with the CVRP have been published, see Eilon and Christofides [1971], Christofides [1985], Golden and Assad [1988], Fischer [1995], Crainic and Laporte [1998], Toth and Vigo [2002], and Golden et al. [2008]. Being a generalization of the TSP, the CVRP is an $\mathcal{N} \mathcal{P}$-hard problem.

The CVRP models real-life problems encountered in the physical distribution of goods and appears in many practical situations, such as the collection of mail from mailboxes, the pickup of children by school buses, house-call tour by a doctor, preventive maintenance inspection tours, the delivery of laundry, etc.

The third problem, referred to as network configuration, is commonly known as uncapacitated facility location problem (UFL). A set of possible locations at which facilities (such as warehouses) may be built are given. For each location, the cost incurred to build the facility is known. The facilities must be built in order to serve a set of customer locations (such as stores). The cost of serving a customer location from each possible facility location is given. The problem is to decide the locations at which to build facilities and to assign each customer to a facility in such a way that the total cost for building facilities and serving customers is minimum.

The earliest works on the UFL problem date back to the 50's and 60's (see Koopmans and Beckmann [1957], Kuehn and Hamburger [1963], Manne [1964], Efroymson and Ray [1966]). Since then, several books were published on the UFL and other variants of location problems, see Love et al. [1988], Mirchandani and Francis [1990], Drezner [1995], Daskin [1995], Farahani and Hekmatfar [2009], Eiselt and Marianov [2011]. It can easily be shown that the UFL problem is $\mathcal{N} \mathcal{P}$-hard. 
The UFL problem has several applications, such as bank account allocation, clustering analysis, lock-box location, economic lot sizing, machine scheduling, design of communication networks, and portfolio management. The problem also appears as a subproblem in several contexts, for example network design, vehicle routing, and, of course, location theory with additional constraints.

In this thesis, we deal with some variants of the three aforementioned problems (TSP, VRP, and UFL). We study the mathematical formulations proposed in the literature and review the state-of-the-art exact algorithms presented so far. Foremost, we propose new models for some of the problems considered and develop new exact algorithms that are highly competitive with the other exact algorithms from the literature. The new algorithms proposed share common ingredients: column generation (see Desaulniers et al. [2005]), dynamic programming (see Bellman [1957], Bertsekas [1995], Bertsekas [2000], Art and Mauch [2007]), and state-space relaxation (see Christofides et al. [1981c], Christofides et al. [1981b]).

\subsection{Reasons for Research}

In the last few decades, optimization packages, based on operations research and mathematical programming techniques, have been used more and more to support managerial decisions in distribution systems. This has been possible for the development of both computer systems (hardware and software) and modeling and algorithmic tools to model and solve real-world instances addressed in the field of logistics.

Today's global markets put enterprises in fierce competition and motivates the continuous evolution of the management of distribution systems.

The study of efficient and innovative methods for supporting managers in taking decisions in all levels (strategic, tactical or operational) is justified by the economical impact of such problems on the firms' revenues. LaLonde and Zinszer [1976] estimated that distribution costs account for around $10 \%$ of the all firms' revenues. The Institute of Logistics and Distribution Management [1985] calculated that distribution costs represent more than $45 \%$ of the total logistics costs, and, in specific cases, like in the soft drink industry, they represent approximately $70 \%$ of the value added costs of goods (see Golden and Wasil [1987]). Recent studies (Eurostat [2009, 2011]) by the statistical office of the European Community (EUROSTAT) estimated that 4,140 billion tonnes of goods per kilometer were moved in 2006, $46 \%$ of which were on roads involving 32.2 million vehicles. Therefore, even small savings in distribution costs may have a relevant global impact.

Another crucial factor that justifies the study of methods to support managerial decisions is the environmental impact of the activity of transporting goods. A total GHG (i.e., greenhouse gas) emission of 992.3 million tonnes of $\mathrm{CO}_{2}$ was attributable 
to transport in the EU-27 in 2006 (see Eurostat [2009]). Indeed, transport made up a share of $19 \%$ of total GHG emissions in the EU-27 - the second largest after the energy industries (31\%). Not surprisingly, road transport is the principal polluter in the transport sector, contributing $93.1 \%$ to the greenhouse gas emissions. Thus, a smart and sustainable transport policy all over EU-27 is fundamental to ensure that the transport systems meet the citizen's economic, social and environmental needs.

\subsection{Contents and Contributions of the Thesis}

In this thesis, we deal with five variants of the three problems (TSP, VRP, and UFL) described in $\S 1.1$ :

- asymmetric traveling salesman problem,

- traveling salesman problem with time windows,

- vehicle routing problem with time windows,

- multi-trip vehicle routing problem,

- two-echelon capacitated vehicle routing problem.

\subsubsection{Asymmetric Traveling Salesman Problem}

Many variants of the TSP have been proposed in the literature. Among them, the asymmetric traveling salesman problem (ATSP) is one of the most studied. The ATSP generalizes the TSP by dropping the assumption that the travel distance between two cities is the same in both directions.

Subsuming the TSP as a special case, the ATSP is $\mathcal{N} \mathcal{P}$-hard, too.

The ATSP can be formally stated as follows. A complete directed graph $G=(V, A)$ is given, where $V$ and $A$ are the vertex set and arc set, respectively. A cost is associated with each $\operatorname{arc}(i, j) \in A$. Let $\mathscr{H}$ be the family of all tours in $G$, where each tour has a cost equal to the sum of the costs of the traversed arcs. The ATSP is to find a tour of the set $\mathscr{H}$ of minimum cost.

There are many important real-life problems that are naturally modeled as ATSPs. In industrial scheduling, the optimal sequencing of jobs on machines with setup times is an ATSP: more generally, the optimal ordering of any set of tasks or operations with sequence dependent changeover costs is an ATSP or one of its generalizations.

Chapter 2 surveys the most effective mathematical models and exact algorithms proposed for solving the ATSP to optimality. Starting from the fundamental integer 
linear programming model of Dantzig et al. [1954], classical relaxations (i.e., assignment, shortest spanning r-arborescence, linear programming) are derived. The most effective branch-and-bound and branch-and-cut exact algorithms from the literature are described. A review of the polynomial formulations and a theoretical comparison of their linear relaxations are provided. The 3-node and 2-node transformations of ATSP instances into symmetric TSP instances are also described. Finally, the considered exact algorithms are experimentally compared on a set of benchmark instances.

\subsubsection{Traveling Salesman Problem with Time Windows}

The traveling salesman problem with time windows (TSPTW) is another well-studied generalization of the TSP, where, other than the travel distance of traveling between two cities, the travel time is known and each city must be visited within a given time interval (time window). Throughout the thesis, whenever we talk of TSPTW, we make no assumptions on the symmetry of travel distances and travel times between cities, so we always consider the general case of asymmetric TSPTW.

Being a generalization of the TSP, the TSPTW is $\mathcal{N} \mathcal{P}$-hard, too. Indeed, finding a feasible solution of asymmetric TSPTWs is $\mathcal{N} \mathcal{P}$-complete (see Savelsbergh [1985]).

The TSPTW is defined on a directed graph $G=(V, A)$. The vertex set $V$ is made up of $n+1$ vertices $0,1, \ldots, n$, where vertex 0 represents the city where the salesman starts and returns and the other $n$ vertices are the cities to visit. For each arc $(i, j)$ of the $\operatorname{arc}$ set $A$, the travel distance and the travel time are known. For each vertex $i \in V$, a time window $\left[e_{i}, l_{i}\right]$ is given. If the salesman arrives at vertex $i$ before the beginning of the time window, $e_{i}$, the visit is postponed until time $e_{i}$. Let $\mathscr{H}$ be the family of all tours in $G$, such that each city is visited within its time window. The TSPTW is to find a tour of the set $\mathscr{H}$ of minimum total distance.

The TSPTW has applications in single and multiple vehicle problems. Practical TSPTWs are encountered in a variety of industrial and service sector applications; examples include control of stacker cranes in warehouses (see Ascheuer et al. [2001]), bank deliveries, postal deliveries, and school-bus routing and scheduling.

In Chapter 3, we describe a new exact algorithm for solving the TSPTW. The fundamental ingredient of the new exact method is state-space relaxation, which is a general relaxation procedure, proposed by Christofides et al. [1981b,c], whereby the state-space associated with a given dynamic programming recursion is relaxed in order to compute valid bounds to the original problem. We propose new tour relaxations, called $n g$ and $n g L$, to compute valid lower bounds on the TSPTW, corresponding to the costs of a problem that seeks a least-cost convex combination of the tours of a given tour relaxation. An optimal TSPTW integer solution is then found by iteratively running a dynamic programming algorithm, that limits the number of states to generate 
by using bounding functions based on the different tour relaxations proposed and on the lower bounds and corresponding dual solutions previously achieved. An extensive computational analysis on basically all TSPTW benchmark instances (with up to 233 vertices) is reported. The new algorithm is compared with the state-of-the-art exact algorithms (i.e., two branch-and-cut algorithms, a dynamic programming recursion, and a constraint-programming-based method). The computational experiments show that the proposed algorithm solves all but one instance to optimality within short computing times and outperforms the other exact methods considered.

\subsubsection{Vehicle Routing Problem with Time Windows}

Real-life routing problems usually include a wide range of operational constraints, so many variants of the basic CVRP have been proposed in the literature. The most studied variant of the CVRP is the vehicle routing problem with time windows (VRPTW).

The VRPTW can be formally defined on a directed graph $G=\left(V^{\prime}, A\right)$, where $V^{\prime}=$ $\{0, \ldots, n\}$ is the vertex set and $A$ is the arc set. A travel cost and a travel time are associated with each $\operatorname{arc}(i, j) \in A$. A set of $m$ identical vehicles, each with capacity $Q$, are available at the depot (represented by vertex 0 ). A nonnegative demand and a time window are associated with each customer $1, \ldots, n$. The VRPTW consists of finding a collection of $m$ simple circuits (each corresponding to a vehicle route) of minimum total cost, defined as the sum of the costs of the arcs belonging to the circuits, such that each circuit visits the depot, each customer is visited by exactly one circuit within its time window, and the sum of the demands of the vertices visited by a circuit does not exceed the vehicle capacity, Q.

The VRPTW generalizes not only the CVRP but also the TSPTW, which can be considered as the single-vehicle version of the VRPTW. Since the CVRP is $\mathcal{N} \mathcal{P}$-hard, by restriction, the VRPTW is also $\mathcal{N} \mathcal{P}$-hard.

The VRPTW has numerous applications in distribution management, such as beverage/food and newspaper delivery. Time windows naturally arise in problems faced by business organizations which work on strict time schedules; specific examples of such problems include bank deliveries, industrial refuse collection, and school-bus routing and scheduling.

Chapter 4 describes an effective exact method for solving the VRPTW that improves on the method proposed by Baldacci et al. [2008] for the CVRP. The new algorithm relies on the set partitioning formulation of the problem and on the state-space relaxations introduced for the TSPTW in Chapter 3. The core of the method is the dual-ascent heuristics developed to find near-optimal dual solutions of the linear relaxation of the set partitioning model; these dual solutions and the corresponding lower 
bounds are computed via column generation by solving the pricing problem with dynamic programming and state-space relaxations and by solving the master problem through subgradient optimization. Another main ingredient of the exact method is a column-and-cut generation algorithm that strengthens the lower bounds achieved by the dual-ascent heuristics by adding valid inequalities; the pricing problem is solved with dynamic programming but adopting a new strategy of combining different feasible dual solutions to limit the state-space graph. An optimal VRPTW integer solution is finally found by solving, with a general purpose integer programming solver, a restricted master problem generated by taking into account all of the feasible dual solutions computed by the different bounding procedures previously run. We show that the proposed algorithm can be properly tailored to obtain an effective solution method for the CVRP. The last part of Chapter 4 compares the performance of our exact method on both VRPTW and CVRP benchmark instances with the state-of-the-art exact algorithms (branch-and-cut and branch-and-cut-and-price) from the literature. The proposed method solves four of the five open Solomon VRPTW instances and significantly improves the average running times of the state-of-the-art algorithms for both the VRPTW and the CVRP.

\subsubsection{Multi-Trip Vehicle Routing Problem}

The multi-trip vehicle routing problem (MTVRP) is a generalization of the CVRP, where each vehicle is allowed to perform a set of routes, called a schedule, of total duration not exceeding a maximum driving time.

The MTVRP is defined on an undirected graph $G=\left(V^{\prime}, E\right)$. The vertex set $V^{\prime}$ is partitioned as $V^{\prime}=\{0\} \cup V$, where vertex 0 represents the depot and the set $V=\{1, \ldots, n\}$ represents $n$ customers, each one requiring $q_{i}$ units of product from the depot. A travel cost and a travel time are associated with each edge $\{i, j\} \in E$. A fleet of $m$ identical vehicles is located at the depot; each vehicle has capacity $Q$ and maximum driving time $T$. The MTVRP calls for the design of a set of $m$ schedules of minimum total cost such that each customer is visited exactly once by the routes of the schedules and each schedule does not exceed the maximum driving time.

The reason for studying the MTVRP is that, in many practical applications, the assumption that a vehicle can perform at most a route per day is unrealistic. This is especially true whenever the vehicle capacity is small with respect to the quantities requested by customers or whenever the planning period is large. In urban areas, for example, travel times are rather small, and it is often the case that after performing short tours vehicles are reloaded and used again. A practical application of MTVRP is the distribution of perishable goods.

Despite its practical importance, little attention has been given to the MTVRP. Though a few heuristics have been proposed in the last two decades, no exact algorithm can be 
found in the literature, except for an exact algorithm proposed for an MTVRP with time windows and unlimited maximum driving time.

In Chapter 5, we present an exact method for solving the MTVRP. The method is based on two set-partitioning-like formulations. The first formulation has a binary variable for each feasible route and each vehicle, whereas the second formulation has a binary variable for each feasible schedule. The linear relaxations of the two formulations and the relation between them are studied. We propose four column-and-cut generation bounding procedures to solve the linear relaxations of the two formulations enforced with valid inequalities derived from the CVRP and with a new class of valid inequalities specific for the problem. Furthermore, we present a simple though effective method to improve the lower bounds achieved by the different bounding procedures that is based on the use of multiple feasible dual solutions. The exact method we propose executes, in sequence, the four bounding procedures and then finds an optimal MTVRP solution by solving, with a general purpose integer programming solver, a restricted master problem containing a reduced set of either routes or schedules belonging to any optimal solution. The computational results show that the proposed method can solve, to optimality, MTVRP benchmark instances involving up to 120 customers.

\subsubsection{Two-Echelon Capacitated Vehicle Routing Problem}

The two-echelon capacitated vehicle routing problem (2E-CVRP) models two-level distribution systems in which freight arrives at a central depot, is transported to intermediate facilities, called satellites, and is finally delivered to customers from the satellites. Therefore, two distribution levels are involved. The distribution system takes into account routing costs, handling costs for the operations of unloading and loading freight at satellites, and fixed costs for using satellites. Limits on the maximum number of first and second level vehicles to use, on the maximum number of vehicles to route at each satellite, and on the maximum quantity of freight to handle at each satellite are imposed. The objective is to minimize the total cost of the system.

The 2E-CVRP concerns both vehicle fleet management and network configuration. Indeed, the 2E-CVRP subsumes both the CVRP and the UFL, so it is an $\mathcal{N} \mathcal{P}$-hard problem.

A common application of the 2E-CVRP is city logistics (see Taniguchi [2001], Taniguchi and Thompson [2008]), which is an area of urban study and urban management. The goal of city logistics is to establish efficient, safe and environmentally friendly urban freight transport systems. Space in city centers is often limited and must be shared between private and public passenger transport as well as parking facilities. Other than producing congestion, pollution and noise, large vehicles usually have low average loads and perform numerous empty trips. Therefore, officials want to reduce the number of 
vehicles, especially freight vehicles, in city centers and want to switch from large to smaller vehicles.

In Chapter 6, we introduce a new mathematical formulation of the 2E-CVRP that is used to derive both integer and continuous relaxations. We present a new bounding procedure based on dynamic programming and an exact algorithm that decomposes the 2E-CVRP into a set of CVRPs with multiple depots and side constraints. The new algorithm is tested on five sets of instances from the literature and a new set of instances with up to 100 customers and 6 satellites. Computational results show that 144 out of 153 instances from the literature were solved, 97 of which for the first time. The comparisons with the state-of-the-art exact methods show that new exact method compares favorably with the other exact methods from the literature. 


\section{Chapter 2}

\section{Asymmetric Traveling Salesman Problem}

1

This chapter surveys the most effective mathematical models and exact algorithms proposed for finding the optimal solution of the well-known asymmetric traveling salesman problem (ATSP). The fundamental integer linear programming (ILP) model proposed by Dantzig, Fulkerson and Johnson is first presented, its classical (Assignment, Shortest Spanning $r$-Arborescence, Linear Programming) relaxations are derived, and the most effective branch-and-bound and branch-and-cut algorithms are described. The polynomial ILP formulations proposed for the ATSP are then presented and analyzed. The considered algorithms and formulations are finally experimentally compared on a set of benchmark instances.

\section{$2.1 \quad$ Introduction}

Let $G=(V, A)$ be a complete digraph, where $V=\{1, \ldots, n\}$ is the vertex set and $A=\{(i, j): i, j \in V\}$ the arc set, and let $c_{i j}$ be a cost associated with $\operatorname{arc}(i, j) \in A$ (with $c_{i i}=+\infty$, for $i \in V$ ). A Hamiltonian circuit (tour) of graph $G$ is a circuit that visits each vertex of the vertex set $V$ exactly once. The asymmetric traveling salesman problem (ATSP) is to find a Hamiltonian circuit $G^{*}=\left(V, A^{*}\right)$ of graph $G$ whose cost $\sum_{(i, j) \in A^{*}} c_{i j}$ is minimum. If the considered graph $G$ is undirected, the corresponding problem is denoted as symmetric traveling salesman problem (STSP).

The ATSP is known to be $\mathcal{N} \mathcal{P}$-hard in the strong sense and has been intensively studied in the last six decades. In this chapter, we consider and experimentally compare the most effective integer linear programming (ILP) models and exact algorithms proposed

\footnotetext{
${ }^{1}$ This chapter is based on Roberti and Toth [2012]
} 
for solving the ATSP. Previous surveys on the subject were presented by Balas and Toth [1985], Fischetti et al. [2004], Öncan et al. [2009], D'Ambrosio et al. [2010]. Several books dealing with the STSP and its variations have been published; among them, we mention those by Lawler et al. [1985], Reinelt [1994], Gutin and Punnen [2002], Applegate et al. [2007].

\subsection{The Dantzig-Fulkerson-Johnson Formulation and its Relaxations}

Dantzig et al. [1954] proposed the following ILP model (hereafter DFJ) with $n^{2}$ binary variables $x_{i j}$

$$
\begin{aligned}
& (D F J) \quad \min \sum_{i=1}^{n} \sum_{j=1}^{n} c_{i j} x_{i j} \\
& \text { s.t. } \sum_{i=1}^{n} x_{i j}=1, \quad j=1, \ldots, n \text {, } \\
& \sum_{j=1}^{n} x_{i j}=1, \quad i=1, \ldots, n, \\
& \sum_{i \in S} \sum_{j \in S} x_{i j} \leq|S|-1, \quad S \subset V: S \neq \varnothing, \\
& x_{i j} \in\{0,1\}, \quad(i, j) \in A \text {, }
\end{aligned}
$$

where variable $x_{i j}$ is equal to 1 if and only if $\operatorname{arc}(i, j) \in A$ is in the optimal tour. Constraints (2.2) and (2.3) impose that the in-degree and out-degree, respectively, of each vertex is equal to one, whereas constraints (2.4) are subtour elimination constraints (SECs) and impose that no partial circuit exists.

Moreover, it is well-known that one can halve the number of SECs (2.4) by replacing them with

$$
\sum_{i \in S} \sum_{j \in S} x_{i j} \leq|S|-1, \quad S \subset V \backslash\{r\}: S \neq \varnothing,
$$

where $r$ is any vertex of vertex set $V$.

Because of constraints (2.2) and (2.3), constraints (2.4) can be equivalently written as connectivity constraints

$$
\sum_{i \in S} \sum_{j \in V \backslash S} x_{i j} \geq 1, \quad S \subset V: S \neq \varnothing
$$


Also in this case, one can halve the number of connectivity constraints (2.6) by replacing them with

$$
\sum_{i \in S} \sum_{j \in V \backslash S} x_{i j} \geq 1, \quad S \subset V: r \in S
$$

or with

$$
\sum_{i \in S} \sum_{j \in V \backslash S} x_{i j} \geq 1, \quad S \subset V: S \neq \varnothing, r \notin S
$$

where $r \in V$ is any fixed vertex.

A valid lower bound on the ATSP can be obtained by optimally solving the linear programming (LP) relaxation of the previous models (2.1)-(2.5) or (2.1)-(2.3) plus constraints (2.5) and (2.7), obtained by replacing constraints (2.5) with constraints

$$
x_{i j} \geq 0, \quad(i, j) \in A \text {. }
$$

Although the considered ILP models require an exponential number of SECs or connectivity constraints, their LP relaxations can be efficiently solved in polynomial time by using the effective polynomial separation procedure proposed by Padberg and Rinaldi [1990a] for the STSP.

Additional lower bounds can be obtained by considering the different substructures of the ATSP, each associated with a subset of constraints defining a well-structured relaxation.

Constraints (2.2), (2.3) and (2.9), with objective function (2.1), define the well-known min-sum assignment problem (AP). Such a problem always has an integer optimal solution and requires the finding of a minimum-cost collection of vertex-disjoint subtours visiting all vertices of graph $G$. Relaxation AP can be solved in $O\left(n^{3}\right)$ time (see, e.g. Lawler [1976], and Carpaneto and Toth [1987] for an efficient implementation).

Constraints (2.2), (2.7) and (2.9), with objective function (2.1), define the well-known shortest spanning r-arborescence problem (r-SAP). Such a problem always has an integer optimal solution and corresponds to finding a minimum-cost spanning subdigraph $\bar{G}=(V, \bar{A})$ of graph $G$ such that (i) the in-degree of each vertex is exactly one, and (ii) each vertex can be reached from the root vertex $r$. Relaxation r-SAP can be solved in $O\left(n^{2}\right)$ time by finding the shortest spanning arborescence rooted at vertex $r$ (see, e.g. Edmonds [1967], Tarjan [1977], and Fischetti and Toth [1993] for an efficient implementation) and adding the minimum-cost arc entering vertex $r$.

A third substructure, corresponding to constraints (2.3), (2.8) and (2.9), with objective function (2.1), defines the shortest spanning r-antiarborescence problem (r-SAAP). Such a problem can easily be transformed into an r-SAP by simply transposing the input cost matrix, so it can be solved in $O\left(n^{2}\right)$ time. Different choices of the root vertex 
$r$ generally produce different values of the lower bounds corresponding to relaxations $\mathrm{r}$ SAP and r-SAAP. Moreover, these relaxations can be strengthened by considering the associated Lagrangean relaxations, obtained by embedding, in a Lagrangean fashion, the relaxed constraints (2.3) for r-SAP, and (2.2) for r-SAAP, in the objective function (2.1). Near-optimal Lagrangean multipliers, leading to good lower bounds, can be obtained by applying the well-known subgradient optimization procedure proposed by Held and Karp [1970] and Held and Karp [1971] for the STSP.

The lower bounds corresponding to relaxations AP, r-SAP and r-SAAP can also be improved (see Fischetti and Toth [1992]) by combining the associated substructures with the additive approach introduced by Fischetti and Toth [1989].

\subsection{Review of Polynomial Formulations}

In this section, we consider the papers presenting polynomial formulations for the ATSP. For each paper, we focus on the formulation producing the tightest LP-relaxation lower bound. Unlike the exact algorithms described in $\S 2.4$, the polynomial formulations can be directly solved by a general-purpose ILP solver. Classifications and comparisons of the polynomial formulations for the ATSP have been recently presented in Öncan et al. [2009] and Godinho et al. [2011].

The earliest polynomial formulation (i.e., formulation requiring a number of constraints polynomial in the number of vertices $n$ ) of the ATSP is owed to Miller et al. [1960] (hereafter MTZ) and is given by (2.1)-(2.3), (2.5) plus the following $(n-1)^{2}$ constraints to break subtours

$$
u_{i}-u_{j}+(n-1) x_{i j} \leq n-2, \quad i, j=2, \ldots, n,
$$

where $u_{i}, i=2, \ldots, n$, is an arbitrary real number representing the order of vertex $i$ in the optimal tour. Miller et al. originally proposed their formulation with no bounds on variables $u_{i}$. Later on, simple bounds (e.g., $1 \leq u_{i} \leq n-1, i=2, \ldots, n$ ) were introduced to restrict the range of variables $u_{i}$. This does not affect the LP bound of MTZ and, in our computational experiments, has shown to increase the computing time, so we leave variables $u_{i}$ unrestricted.

Gavish and Graves [1978] proposed another formulation (hereafter GG) having LP relaxation stronger than that of MTZ (see Wong [1980] and Padberg and Sung [1991]) but weaker than that of DFJ (see Gouveia [1995]). Formulation GG is a single-commodity flow formulation where subtours are broken by introducing $n^{2}-n$ nonnegative variables $g_{i j}, i=2, \ldots, n, j=1, \ldots, n$. Formulation GG consists of constraints (2.1)-(2.3), 
(2.5) and the following constraints

$$
\begin{aligned}
& \sum_{j=1}^{n} g_{i j}-\sum_{j=2}^{n} g_{j i}=1, \\
& 0 \leq g_{i j} \leq(n-1) x_{i j}, \quad i=2, \ldots, n, j=1, \ldots, n,
\end{aligned}
$$

where variables $g_{i j}$ can be interpreted as the number of arcs on the path from vertex 1 to arc $(i, j)$ in the optimal tour (see Gouveia and Pires [1999]).

For fixed values of the variables $x_{i j}$, constraints (2.11) and (2.12) form a network flow problem, so variables $g_{i j}$ take integer values. Langevin et al. [1990] showed that the LP relaxation of GG is equivalent to that of the two-commodity flow formulation proposed by Finke et al. [1984] - hereafter FCG.

Fox et al. [1980] proposed three formulations for the time-dependent traveling salesman problem that are valid for the ATSP, as well. These formulations present $n^{3}$ binary variables, $r_{i j k}$, that are equal to 1 if and only if $\operatorname{arc}(i, j) \in A$ is in position $k$ in the optimal tour. The first formulation (therein P1) has $4 n$ constraints, whereas the second formulation (hereafter P1b) has $3 n$ constraints and is obtained from the first one by dropping a set of $n$ constraints. Thus, the LP relaxation of the first formulation is stronger than that of the second one; Gouveia and Voß [1995] showed that the LP relaxations of both formulations are stronger than that of GG. The third formulation proposed by Fox et al. (therein P2) has $n+1$ constraints and is obtained from the first formulation by surrogating the first $3 n$ constraints. Öncan et al. [2009] showed that the LP relaxation of the third formulation is weaker than that of the second one, whereas Padberg and Sung [1991] proved that the LP relaxation of the third formulation is also weaker than that of DFJ. We have not reported the three formulations because, in our computational experiments, they proved to be ineffective in solving the ATSP.

The first multi-commodity flow (MCF) formulation was proposed by Wong [1980] (hereafter WONG). WONG considers $2 n-2$ commodities and introduces $2 n^{3}-2 n^{2}$ nonnegative continuous variables and $4 n^{3}-2 n^{2}-2 n$ constraints. This formulation was later modified by Langevin [1988] and by Loulou [1988] to obtain two additional MCF formulations which provide LP relaxations equivalent to that of WONG.

Another MCF formulation with only $n-1$ commodities was proposed by Claus [1984]. This formulation (hereafter CLAUS) introduces $n^{3}-n^{2}$ nonnegative continuous variables $w_{i j}^{k}, i, j=1, \ldots, n, k=2, \ldots, n$, and $2 n^{3}-n^{2}-n$ constraints, and consists of 
constraints (2.1)-(2.3), (2.5) plus the following constraints to break subtours

$$
\begin{array}{lr}
\sum_{j=1}^{n} w_{i j}^{k}-\sum_{j=1}^{n} w_{j i}^{k}=0, & i, k=2, \ldots, n: i \neq k, \\
\sum_{j=2}^{n} w_{1 j}^{k}-\sum_{j=2}^{n} w_{j 1}^{k}=-1, & k=2, \ldots, n, \\
\sum_{j=1}^{n} w_{i j}^{i}-\sum_{j=1}^{n} w_{j i}^{i}=1, & i=2, \ldots, n, \\
0 \leq w_{i j}^{k} \leq x_{i j}, & i, j=1, \ldots, n, k=2, \ldots, n,
\end{array}
$$

where variable $w_{i j}^{k}$ is equal to 1 if and only if the commodity going from vertex 1 to vertex $k$ flows on arc $(i, j)$. Langevin et al. [1990] proved that the LP relaxation of CLAUS is equivalent to that of WONG, whereas Padberg and Sung [1991] proved that the LP relaxation of CLAUS is equivalent to that of DFJ, as well.

Formulation MTZ was strengthened by Desrochers and Laporte [1990], who proposed formulation DL having LP relaxation stronger than that of MTZ and obtained from MTZ by replacing constraints (2.10) with the following $n^{2}-1$ lifted constraints

$$
\begin{array}{lc}
u_{i}-u_{j}+(n-1) x_{i j}+(n-3) x_{j i} \leq n-2, & i, j=2, \ldots, n, \\
-u_{i}+(n-3) x_{i 1}+\sum_{j=2}^{n} x_{j i} \leq-1, & i=2, \ldots, n, \\
u_{i}+(n-3) x_{1 i}+\sum_{j=2}^{n} x_{i j} \leq n-1, & i=2, \ldots, n .
\end{array}
$$

Gouveia and Pires [1999] presented four formulations (therein called RMTZ, L1RMTZ, L2RMTZ, and L3RMTZ). The LP relaxations of all of these formulations are stronger than that of MTZ. Here, we present formulation L3RMTZ (hereafter GP), whose LP relaxation is stronger than those of L1RMTZ and L2RMTZ, which in turn are stronger than that of RMTZ. Gouveia and Pires [1999] also showed that the LP relaxations of formulations L1RMTZ and L2MTZ are weaker than those of MCF formulations, such as WONG and CLAUS. Formulation GP introduces $(n-1)^{2}$ additional nonnegative continuous variables $v_{i j}, i, j=2, \ldots, n$, that are equal to 1 if and only if vertex $i$ is in the path from vertex 1 to vertex $j$. Formulation GP consists of constraints (2.1)-(2.3), (2.5) plus the following $2 n^{3}-10 n^{2}+18 n-10$ constraints to break subtours

$$
\begin{array}{lr}
x_{i j}-v_{i j} \leq 0, & i, j=2, \ldots, n, \\
x_{i j}+v_{j i} \leq 1, & i, j=2, \ldots, n, \\
x_{j i}+x_{i j}+v_{k i}-v_{k j} \leq 1, & i, j, k=2, \ldots, n: i \neq j \neq k, \\
x_{k j}+x_{i k}+x_{i j}+v_{k i}-v_{k j} \leq 1, & i, j, k=2, \ldots, n: i \neq j \neq k .
\end{array}
$$


Gouveia and Pires [2001] presented other formulations, among them a polynomial formulation (therein $\mathrm{MCF}+$ ) whose LP relaxation is stronger than those of CLAUS and GP. As the LP relaxation of MCF + is weaker than that of formulation SST (which will be introduced later in this section), as shown by Öncan et al. [2009], and MCF+ has more constraints than SST, we do not report a detailed description of MCF+.

Sherali and Driscoll [2002] strengthened formulation DL by applying a reformulationlinearization technique and by introducing $(n-1)^{2}$ additional nonnegative continuous variables $y_{i j}, i, j=2, \ldots, n$, where variable $y_{i j}$ represents the order of arc $(i, j)$ in the optimal tour. The resulting formulation (hereafter SD) replaces constraints (2.13), (2.14) and (2.15) with the following $4 n^{2}-4 n$ constraints

$$
\begin{array}{lr}
\sum_{j=2}^{n} y_{i j}+(n-1) x_{i 1}-u_{i}=0, & i=2, \ldots, n, \\
\sum_{i=2}^{n} y_{i j}-u_{j}=-1, & j=2, \ldots, n, \\
x_{i j}-y_{i j} \leq 0, & i, j=2, \ldots, n, \\
y_{i j}-(n-2) x_{i j} \leq 0, & i, j=2, \ldots, n, \\
u_{j}+(n-2) x_{i j}+(n-1) x_{j i}-y_{i j}-y_{j i} \leq n-1, & i, j=2, \ldots, n, \\
y_{i j}+y_{j i}-u_{j}-x_{j i} \leq-1, & i, j=2, \ldots, n, \\
-x_{1 j}+(n-3) x_{j 1}-u_{j} \leq-2, & j=2, \ldots, n, \\
(n-3) x_{1 j}-x_{j 1}+u_{j} \leq n-2, & j=2, \ldots, n .
\end{array}
$$

Recently, Öncan et al. [2009] showed that the LP relaxation of SD is also stronger than that of GG.

Sarin et al. [2005] studied the asymmetric traveling salesman problem with and without precedence constraints and proposed five polynomial formulations (therein ATSPxy, L1ATSPxy, SL1ATSPxy, L2ATSPxy and ML1ATSPxy) for the ATSP, whose LP relaxations are stronger than that of RMTZ. Moreover, Sarin et al. [2005] showed that the LP relaxation of L1ATSPxy is stronger than that of SL1ATSPxy whose LP relaxation is stronger than that of ATSPxy; the LP relaxations of L1ATSPxy and L2ATSPxy are also stronger than those of formulations L1RMTZ and L2RMTZ by Gouveia and Pires [1999], respectively. Here we report formulation L2ATSPxy (hereafter SSB) only, which, in our computational experiments, was shown to be the best performer. Formulation SSB introduces $(n-1)^{2}$ nonnegative continuous variables $d_{i j}, i, j=2, \ldots, n$, and $n^{3}-n^{2}-n+1$ constraints, and consists of constraints (2.1)-(2.3), (2.5) and the 
following constraints to break subtours

$$
\begin{array}{lr}
d_{i j}-x_{i j} \geq 0, & i, j=2, \ldots, n, \\
d_{i j}+d_{j i}=1, & i, j=2, \ldots, n: i \neq j, \\
x_{1 j}+x_{j 1} \leq 1, & j=2, \ldots, n, \\
x_{i j}+d_{j k}+x_{k j}+d_{k i}+x_{i k} \leq 2, & i, j, k=2, \ldots, n .
\end{array}
$$

Although variable $d_{i j}, i, j=2, \ldots, n$, is continuous, it has a binary connotation and is equal to 1 if and only if vertex $i$ precedes (not necessarily immediately) vertex $j$ in the optimal tour. Godinho et al. [2011] noticed that the meaning of variables $d_{i j}$ is basically the same of variables $v_{i j}$ introduced in formulation GP. An analysis of all the formulations involving variables $x_{i j}$ and $v_{i j}$ (or $d_{i j}$ ) can be found in Gouveia and Pesneau [2006].

Sherali et al. [2006] proposed several polynomial formulations. Here, we present formulation SST (therein ATSP6) only, which uses $(n-1)^{3}$ nonnegative continuous variables $t_{i j}^{k}, i, j, k=2, \ldots, n$, and consists of (2.1)-(2.3), (2.5), (2.17) and the following constraints

$$
\begin{array}{lr}
d_{i j}+x_{j i}+d_{j k}+d_{k i} \leq 2, & i, j, k=2, \ldots, n, \\
d_{i j}-x_{1 i} \geq 0, & i, j=2, \ldots, n, \\
d_{j i}-x_{i 1} \geq 0, & i, j=2, \ldots, n, \\
0 \leq t_{i j}^{k} \leq x_{i k}, & i, j, k=2, \ldots, n: i \neq j \neq k, \\
x_{i j}+\sum_{k=2 ; k \neq j}^{n} t_{i j}^{k}=d_{i j}, & i, j=2, \ldots, n, \\
x_{1 k}+\sum_{i=2 ; i \neq j}^{n} t_{i j}^{k}=d_{k j}, & k, j=2, \ldots, n,
\end{array}
$$

where $t_{i j}^{k}$ is equal to 1 if and only if, in the optimal tour, arc $(i, k)$ is used and vertex $k$ precedes vertex $j$. Godinho et al. [2011] pointed out that variables $t_{i j}^{k}$ can be interpreted in the same way as the flow variables $w_{i j}^{k}$ used in formulation CLAUS. Öncan et al. [2009] showed that the LP relaxation of SST is stronger than those of $\mathrm{MCF}+$, whereas Sherali et al. [2006] proved that the LP relaxation of SST is stronger than that of L1ATSPxy.

In Figure 2.1, we summarize the relationships among the linear relaxations of the polynomial formulations reviewed in this section. A link going from formulation A to formulation $\mathrm{B}$ means that the LP relaxation of $\mathrm{B}$ is stronger than that of $\mathrm{A}$, whereas a dashed line connecting two formulations means that the relative LP relaxations are equivalent. 
\begin{tabular}{|l}
$\mathrm{A}$ \\
$\mathrm{A}$
\end{tabular} $\mathrm{B}$ is stronger than $\mathrm{A}$

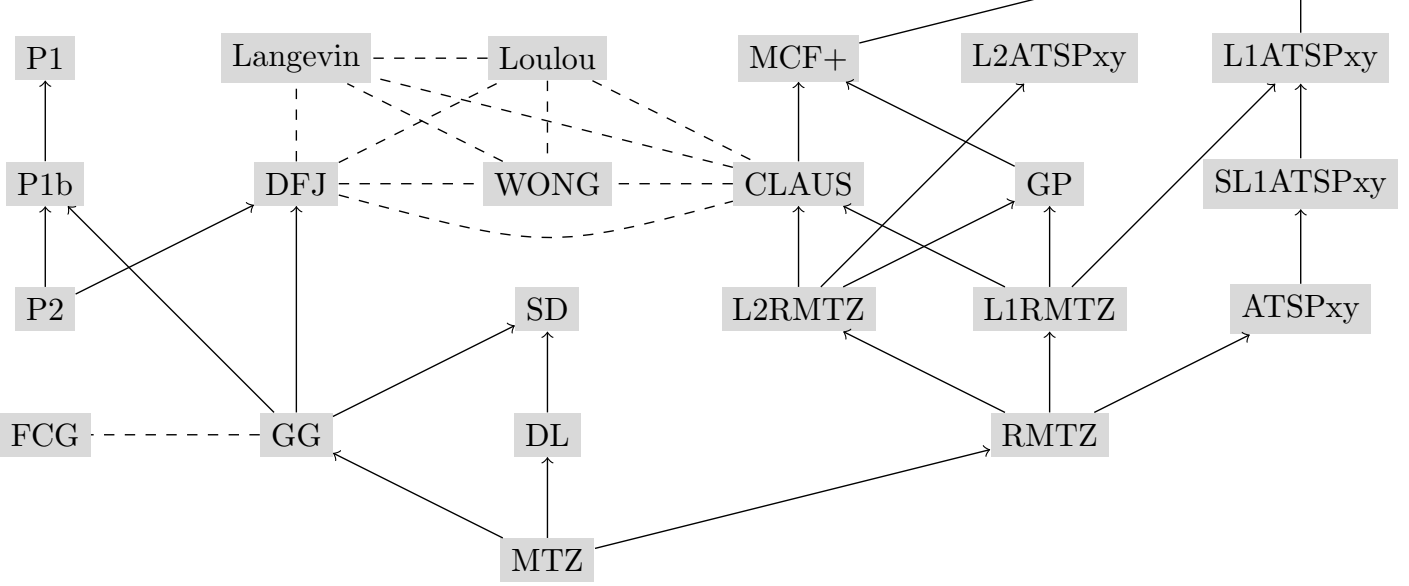

FiguRE 2.1: Relations among the linear relaxations of the polynomial formulations and of formulation DFJ

\subsection{Exact Algorithms}

Many branch-and-bound algorithms have been proposed to find the optimal solution of the ATSP. After the seminal paper by Little et al. [1963], where for the first time the term "branch-and-bound" was coined, other algorithms were proposed by Bellmore and Malone [1971], Garfinkel [1973], Smith et al. [1977], Carpaneto and Toth [1980], Balas and Christofides [1981], Miller and Pekny [1989], Pekny and Miller [1992], Fischetti and Toth [1992], Carpaneto et al. [1995]. In the following, two of the most effective branch-and-bound algorithms for the ATSP (i.e., those proposed by Carpaneto et al. [1995] and Fischetti and Toth [1992]), are briefly reviewed. The algorithm proposed by Pekny and Miller [1992] exhibits, on the whole, a performance comparable with that of the approach described in Carpaneto et al..

The algorithm proposed by Carpaneto et al. [1995] is a lowest-first branch-and-bound method based on the AP relaxation and the subtour elimination branching scheme. At the root node of the decision tree, the AP relaxation of the original problem is solved, the patching heuristic algorithm proposed by Karp [1979] is applied to determine an initial tour of cost $z^{*}$, and a reduction procedure based on the AP reduced costs, $c_{i j}^{\prime}$, is executed to transform the original complete graph into a sparse one (by setting $x_{i j}=0$ if $V(A P)+c_{i j}^{\prime} \geq z^{*}$, where $V(A P)$ is the value of the optimal solution of the AP relaxation). At each of the other nodes of the decision tree, the AP relaxation of the subproblem associated with the considered node is solved, through an effective parametric technique, in $O\left(n^{2}\right)$ time. If $V(A P) \geq z^{*}$, the node is fathomed. Otherwise, if the AP solution contains no subtour (i.e., a feasible solution has been found) the best solution thus far is updated, $z^{*}$ is set equal to $V(A P)$, and the node is fathomed. If neither of the two previous cases occur, the subtour elimination branching scheme 
proposed by Carpaneto and Toth [1980] is applied: the subtour $S$ of the AP solution having the minimum number, say $h$, of not "imposed" arcs is selected, and $h$ descending nodes are generated so as to forbid, by "imposing" and "excluding" proper arc subsets, subtour $S$ for each descending node.

The algorithm proposed by Fischetti and Toth [1992] is a lowest-first branch-and-bound method based on the branching scheme introduced by Carpaneto and Toth [1980], and, at each node of the decision tree, computes the corresponding lower bound by applying the additive approach combining the AP, r-SAP, and r-SAAP relaxations.

More recently, two effective branch-and-cut algorithms for the ATSP have been proposed by Fischetti and Toth [1997] and Fischetti et al. [2003].

The algorithm proposed by Fischetti and Toth [1997] is based on the DFJ model (2.1)-(2.5) and exploits additional classes of facet-inducing inequalities for the ATSP polytope that proved to be of crucial importance for the solution of some real-world instances.

An ATSP inequality $\alpha x \leq \alpha_{0}$ is called symmetric when $\alpha_{i j}=\alpha_{j i}$ for each $\operatorname{arc}(i, j) \in A$. Symmetric inequalities can be thought of as derived from valid inequalities for the STSP defined on the complete undirected graph $G^{\prime}=(V, E)$. Indeed, let $y_{e}$ be equal to 1 if edge $e \in E$ belongs to the optimal STSP solution, $y_{e}=0$ otherwise. Every inequality $\sum_{e \in E} \alpha_{e} y_{e} \leq \alpha_{0}$ valid for the STSP can be transformed into a valid ATSP inequality by simply replacing $y_{e}$ with $x_{i j}+x_{j i}$ for all edges $e=\{i, j\} \in E$. This produces the symmetric inequality $\alpha x \leq \alpha_{0}$, where $\alpha_{i j}=\alpha_{j i}=\alpha_{(i, j)}$ for all couples of vertices $i, j \in V, i \neq j$. Conversely, every symmetric ATSP inequality $\alpha x \leq \alpha_{0}$ corresponds to the valid STSP inequality $\sum_{\{i, j\} \in E} \alpha_{i j} y_{(i, j)} \leq \alpha_{0}$. The above correspondence implies that every separation algorithm for the STSP can be used, as a "black box", for the ATSP, as well. Several exact/heuristic separation algorithms for the STSP have been proposed in recent years, all of which can be used for the ATSP. Only two such separation tools are used in Fischetti and Toth [1997], namely (i) the Padberg and Rinaldi [1990a] exact algorithm for SECs; and (ii) the simplest heuristic scheme for 2-matching constraints, i.e., for combs with 2-node teeth, where each component $H$ of the graph induced by the edges $e \in E$ with fractional $y_{e}^{*}$ is heuristically considered, in turn, as the handle of the comb. Having fixed $H$, the most violated 2-matching constraint with handle $H$ is easily found by sorting the edges having one extreme node in $H$ and the other extreme node in $V \backslash H$ by non increasing $y_{e}^{*}$, and by taking the first $k$ such edges to act as teeth, for $k=1,3,5, \ldots$

In addition, Fischetti and Toth [1997] considered the $D_{k}^{+}$and $D_{k}^{-}$inequalities proposed by Grötschel and Padberg [1985] and the odd close alternative trail (odd CAT) inequalities proposed by Balas [1989] (and analyzed by Fischetti [1991]). The separation problem for the classes of the $D_{k}^{+}$and $D_{k}^{-}$inequalities is a combinatorial optimization problem that can be effectively solved in practice by an implicit enumeration scheme 
enhanced by suitable pruning conditions (see Fischetti and Toth [1997]). As for the detection of violated odd CAT inequalities, Balas [1989] showed that these inequalities correspond to odd cycles in an auxiliary undirected "incompatibility" graph. An effective heuristic separation algorithm, based on the computation of a minimum-weight odd cycle going through a given edge, was proposed by Fischetti and Toth [1997]. In addition, clique lifting (see Fischetti and Balas [1993]) and shrinking (see Padberg and Rinaldi [1990b]) procedures are applied to simplify the considered separation problems. A detailed analysis of the polyhedral structure of the ATSP can be found in Balas and Toth [1985].

Pricing is an important ingredient of branch-and-cut codes because it allows one to effectively handle LP relaxations involving a huge number of variables. In order to keep the size of the LP relaxation as small as possible, the following pricing scheme is commonly used. We determine a (small) core set of arcs, say $\tilde{A}$, and decide to temporarily fix $x_{i j}=0$ for each $\operatorname{arc}(i, j) \in A \backslash \tilde{A}$. We then solve the corresponding restricted LP problem, compute the associated LP reduced costs $\bar{c}_{i j}$ and check whether $\bar{c}_{i j} \geq 0$ for all $(i, j) \in A \backslash \tilde{A}$. If this is the case, then the LP relaxation has been solved to optimality. Otherwise, the current core set $\tilde{A}$ is enlarged by adding (some of) the arcs with negative reduced cost, and the whole procedure is iterated.

Fischetti and Toth [1997] proposed an improved pricing technique, called AP pricing, in which the pricing condition is strengthened by exploiting the fact that any feasible solution of the current LP relaxation cannot arbitrarily select the arcs of negative reduced cost because the degree equations - among other constraints - must be fulfilled.

The exact algorithm proposed by Fischetti and Toth [1997] is a lowest-first branchand-cut method. At each node of the branching tree, the LP relaxation is initialized by taking all of the constraints of the last LP solved at the father node (for the root node, only the degree equations are taken). As for the variables, one retrieves, from a scratch file, the optimal basis associated with the last LP solved at the father node and initializes the core variable set, $\tilde{A}$, by taking the arcs belonging to this basis (for the root node, $\tilde{A}$ contains the $2 n-1$ variables in the optimal AP basis found by solving AP on the original $\operatorname{costs} c_{i j}$ ). In addition, $\tilde{A}$ contains all the arcs of the best known ATSP solution. Starting with the above advanced basis, one iteratively solves the current LP relaxation, applies the AP pricing procedure and repeats if needed.

On exit of the pricing loop, the separation algorithms are applied to find, if any, ATSP inequalities that cut off the current LP optimal solution $x^{*}$. When violated cuts are found, one adds them to the current LP relaxation and repeats.

When separation fails and $x^{*}$ is integer, the current best ATSP solution is updated, and a backtracking step occurs. If $x^{*}$ is fractional, the current LP basis is saved in a file, and one branches on the variable $x_{i j}$ with $0<x_{i j}^{*}<1$ that maximizes the score $\sigma(i, j)=c_{i j} \min \left\{x_{i j}^{*}, 1-x_{i j}^{*}\right\}$. As a heuristic rule, a large priority is given to the 
variables with $0.4 \leq x_{i j}^{*} \leq 0.6$ (if any) so as to produce a significant change in both descending nodes.

This branching scheme has been enhanced by Fischetti et al. [2003] through the fractional persistency mechanism proposed by Fischetti et al. [2001] for the solution of crew scheduling and vehicle scheduling problems. The corresponding branch-and-cut algorithm will be denoted as FLT in $§ 2.6$.

\subsection{Transformation of ATSP Instances into STSP Instances}

Any code for the ATSP can be invoked to solve STSP instances. In fact, the reverse also stands by means of the following two transformations.

- The 3-node transformation proposed by Karp [1972]. A complete undirected graph with $3 n$ vertices is obtained from the original complete directed one by adding two copies, $n+i$ and $2 n+i$, of each vertex $i \in V$, and by (i) setting to 0 the cost of edges $\{i, n+i\}$ and $\{n+i, 2 n+i\}$ for each vertex $i \in V$, (ii) setting the cost of edge $\{2 n+i, j\}$ to $c_{i j}$, for each couple of vertices $i, j \in V$, and (iii) setting to $+\infty$ the costs of all remaining edges;

- The 2-node transformation proposed by Jonker and Volgenant [1983] (see also Jünger et al. [1995]). A complete undirected graph with $2 n$ vertices is obtained from the original complete directed one by adding a copy, $n+i$, of each vertex $i \in V$, and by (i) setting to 0 the cost of the edge $\{i, n+i\}$ for each vertex $i \in V$, (ii) setting the cost of edge $\{n+i, j\}$ to $c_{i j}+M$, for each couple of vertices $i, j \in V$, where $M$ is a sufficiently large positive value, and (iii) setting to $+\infty$ the costs of all the remaining edges. The transformation value $n M$ must be subtracted from the STSP optimal cost.

The most effective branch-and-cut algorithm for the STSP is currently the one by Applegate et al. [2007], and the corresponding code (Concorde by Applegate et al. [1999]), is publicly available. In Fischetti et al. [2004], this code was used to test the effectiveness of the approach based on the ATSP-to-STSP transformation. The code has been used with default parameters. The results have shown that the 2-node transformation is, in general, more effective than the 3-node one.

\subsection{Computational Results}

As a testbed for our computational experiments, we took the 27 ATSP instances collected in the TSPLIB by Reinelt [1991] and 5 real-world instances provided by Balas [2000]. All instances have integer nonnegative costs. 
TABLE 2.1: Computing times for solving the LP relaxations of 8 polynomial formulations by using the primal, dual or barrier method

\begin{tabular}{lrrrrrrrrr}
\hline & \multicolumn{2}{c}{ Primal } & & \multicolumn{2}{c}{ Dual } & & \multicolumn{2}{c}{ Barrier } \\
\cline { 2 - 3 } Formulation & Solved & $T_{L P}$ & & Solved & $T_{L P}$ & & Solved & $T_{L P}$ \\
\hline MTZ & 10 & 0.16 & & 10 & 0.03 & & 10 & 16.30 \\
GG & 10 & 0.25 & & 10 & 0.56 & & 10 & 0.14 \\
CLAUS & 3 & $1,018.11$ & & 10 & 353.84 & & 10 & 95.05 \\
DL & 10 & 0.30 & & 10 & 0.05 & & 10 & 17.23 \\
GP & 10 & 48.01 & & 10 & 3.48 & & 10 & 232.78 \\
SD & 10 & 7.45 & & 10 & 5.10 & & 10 & 0.75 \\
SSB & 9 & 360.49 & & 10 & 6.68 & & 10 & 103.59 \\
SST & 0 & $1,200.00$ & & 1 & $1,128.43$ & & 3 & $1,004.75$ \\
\hline
\end{tabular}

Tables 2.1 and 2.2 report on the computational performance of eight polynomial formulations (namely, MTZ, GG, CLAUS, DL, GP, SD, SSB, and SST) for the ATSP, described in $\S 2.3$, on 10 small-size instances from the TSPLIB (namely, instances ftv33, ftv35, ftv38, ftv44, ftv47, ftv55, ftv64, ftv70, ft70, and ft53), which is the set of instances used in the computational study of Öncan et al. [2009]. The computational results reported in Tables 2.1 and 2.2 were obtained on an Intel Core2 Duo@2.26 GHz by running CPLEX11.2 as LP and ILP solver. All computing times reported in the tables are expressed in seconds.

In Table 2.1, we compare, on the ten considered instances, the performance of the eight polynomial formulations for computing the corresponding LP relaxations by using three different LP methods (namely Primal, Dual and Barrier). For each method and each formulation, Table 2.1 reports the number of LP-bounds computed within the time limit of 1, 200 seconds (column Solved) and the average computing time (column $T_{L P}$ ). In the computation of the average computing time, we considered a computing time of 1,200 seconds whenever the LP relaxation of an instance could not be computed within the time limit or the instance could not be run due to exceeded memory limits for CPLEX.

Table 2.1 shows that, for a given formulation, the computing time may significantly vary by using different LP methods. The dual method turned out to be the best performing method when solving MTZ, DL, GP, and SSB, whereas the barrier method was the best performing one when solving GG, CLAUS, SD, and SST. All formulations but SST could solve the LP relaxation of all 10 instances within the time limit imposed.

Table 2.2 reports, for each of the eight formulations and each of the ten instances, the percentage gap (computed with respect to the optimal solution value) left after solving the LP relaxation (column $L P$ ), the percentage gap left at the root note of the search tree of CPLEX (column $R t$ ), and the computing times for solving the LP relaxation (column $T_{L P}$ ), the root node (column $T_{R t}$ ), and the ILP formulation itself (column $T_{I L P}$ ). For each formulation, we used the LP-method that turned out to be 
the best one in the experiments summarized in Table 2.1. The time limit for solving the ILP formulation was set equal to 1,800 seconds. In the rows labeled "Avg", Table 2.2 reports average values and, in parenthesis, the number of instances solved to optimality within the time limit. In the computation of the average computing times, we considered a computing time equal to 1,200 or 1,800 seconds whenever the LP model or the ILP model, respectively, could not be solved within the time limit. For formulation SST the averages refer to the first three instances since the remaining ones could not be run due to exceeded memory limits for CPLEX.

As to the LP-bounds, Table 2.2 shows that SST obtains tight lower bounds but requires larger computing times than the other formulations. Moreover, SST was able to provide the lower bounds, within the time limit, on only 3 of the 10 instances considered. Among the other formulations, the one that provides the tightest LP-bounds is CLAUS, followed by SSB and GP. The other four formulations (i.e., MTZ, GG, DL and $\mathrm{SD})$ obtain, on average, worse LP-bounds than the previous ones but within shorter computing times (less than a second on average). GG was the only formulation able to solve, to optimality, all of the 10 instances within the time limit imposed, whereas MTZ, DL and SD could solve, to optimality, all but one of the instances. By considering the results reported in Table 2.2, we can conclude that, for what concerns the overall computing time, the most effective formulations are MTZ, GG and DL.

The results reported in Table 2.2 show that formulations MTZ, GG, and DL are the best formulations to be directly used within CPLEX. This is probably due to two main reasons: (i) the limited number of constraints required to break subtours allows CPLEX to compute the LP-bounds effectively, and (ii) the large variety of cuts embedded in CPLEX lead to root lower bounds competitive with those that can be obtained with other formulations having stronger LP-bounds but within much shorter computing times. We stress that the computational analysis reported in Table 2.2 is aimed at comparing the suitability of each formulation to be directly solved with CPLEX. Therefore, any conclusion that can be drawn from the results reported in Table 2.2 cannot consider the fitness of each polynomial formulation as the starting point for more complicated exact algorithms for solving the ATSP. We also stress that, for each class of formulations, we report only the results obtained by the formulation providing, on average, the tightest LP-bounds because the other formulations (i.e., those appearing in Figure 2.1 but neglected in this section) were computationally outperformed by at least one of the eight formulations considered in this study.

In Table 2.3, we compare, of the 27 ATSP instances from TSPLIB and the 5 instances provided by Balas, the performance of (a) the branch-and-bound algorithm by Carpaneto et al. [1995] (hereafter CDT) described in $\S 2.4$, (b) the branch-and-bound algorithm based on the additive bounding procedure by Fischetti and Toth [1992] (hereafter FT) described in $\S 2.4$, (c) the branch-and-cut algorithm by Fischetti et al. [2003] (hereafter FLT) described in $§ 2.4$, (d) the branch-and-cut algorithm (hereafter 
Concorde) solving the STSP instances obtained from the transformation described in $\S 2.5$, and (e) the polynomial formulations MTZ, GG and DL (see §2.3). For each instance and each algorithm, we report the percentage gap of the corresponding lower bound, or the value of the LP relaxation of the considered formulation, computed at the root node of the decision tree (column $L B$ ) and the computing time for solving the instance to optimality (column $T$ ). The rows labeled "Avg" report average values and, in parenthesis, the number of instances solved to optimality within the corresponding time limit.

Different time limits were imposed on the algorithms: 1, 000 seconds for CDT and FT, 10, 000 seconds for FLT and Concorde, 1, 800 seconds for MTZ, GG and DL. For this reason, in the computation of the average computing time, only the instances solved to optimality within the imposed time limit are considered. The rows labeled "Avg 1,000 sec" report the average computing time and, in parenthesis, the number of instances solved to optimality within the time limit of 1,000 seconds (a computing time equal to 1,000 seconds is considered when this time limit is reached).

The computational results reported for CDT, FT, FLT and Concorde are taken from Fischetti et al. [2004] and were obtained on a Digital Alpha $533 \mathrm{MHz}$ with CPLEX6.5.3 as LP solver, whereas the computational results relative to formulations MTZ, GG and DL were obtained on an Intel Core2 Duo@2.26 GHz by running CPLEX11.2. We have experimentally found that the latter machine is approximately 10-12 times faster than that used by Fischetti et al.; therefore, the computing times reported for MTZ, GG and DL were multiplied by 10 in order to have a fair comparison.

Table 2.3 shows that FLT and Concorde were the only two exact methods able to solve, within the imposed time limit, all of the 32 instances to optimality and are clearly better performing than the other 5 exact methods considered. Although, on the considered instances, Concorde generally obtains better lower bounds at the root node, FLT is always faster. Formulations MTZ, GG and DL proved not to be competitive with either FLT or Concorde because they solved only 20, 11 and 19 instances, respectively, and, on the solved instances, their computing times are always much longer. As for the branch-and-bound algorithms CDT and FT, for which a time limit of 1,000 seconds was imposed, it can be noted that they dominate, with regards to the instances solved to optimality within this time limit and the computing times, the polynomial formulations MTZ, GG and DL. By considering the values of the lower bounds at the root node, Table 2.3 shows that: (i) the lower bound of CDT (given by the value of the AP relaxation) is only slightly worse than that of MTZ (i.e., the addition of constraints (2.10) to the AP relaxation only marginally improves the corresponding LP relaxation); (ii) the lower bound of FT (corresponding to the additive bounding procedure) is always better that those of MTZ and GG, and globally better than that of DL. 
TABLE 2.2: Performance of 8 polynomial formulations on 10 ATSP instances from the TSPLIB

\begin{tabular}{|c|c|c|c|c|c|c|c|c|c|c|c|c|c|c|c|c|c|c|c|c|}
\hline \multirow[b]{2}{*}{ Inst } & \multicolumn{5}{|c|}{$M T Z$} & \multicolumn{5}{|c|}{$G G$} & \multicolumn{5}{|c|}{$C L A U S$} & \multicolumn{5}{|c|}{$D L$} \\
\hline & $L P$ & $T_{L P}$ & $R t$ & $T_{R t}$ & $T_{I L P}$ & $L P$ & $T_{L P}$ & $R t$ & $T_{R t}$ & $T_{I L P}$ & $L P$ & $T_{L P}$ & $R t$ & $T_{R t}$ & $T_{I L P}$ & $L P$ & $T_{L P}$ & $R t$ & $T_{R t}$ & $T_{I L P}$ \\
\hline ftv33 & 7.64 & 0.02 & 2.77 & 0.22 & 4.91 & 7.03 & 0.06 & 0.78 & 0.83 & 5.51 & 0.00 & 7.20 & 0.00 & 9.44 & 14.88 & 5.35 & 0.03 & 4.58 & 0.11 & 3.40 \\
\hline ftv35 & 6.12 & 0.02 & 4.15 & 0.09 & 6.27 & 5.59 & 0.06 & 0.63 & 3.51 & 11.93 & 1.06 & 9.17 & 1.00 & 19.02 & 79.36 & 4.04 & 0.05 & 3.58 & 0.16 & 5.23 \\
\hline $\mathrm{ftv} 38$ & 5.87 & 0.02 & 2.91 & 0.14 & 9.02 & 5.44 & 0.08 & 0.80 & 2.29 & 21.46 & 1.02 & 12.50 & 0.96 & 31.01 & 74.88 & 3.45 & 0.03 & 3.14 & 0.17 & 5.71 \\
\hline $\mathrm{ftv} 44$ & 5.55 & 0.02 & 2.68 & 0.14 & 16.40 & 5.18 & 0.10 & 1.74 & 2.47 & 18.02 & 1.74 & 23.71 & 1.27 & 77.38 & $\mathrm{tl}$ & 2.43 & 0.03 & 2.10 & 0.20 & 3.17 \\
\hline $\mathrm{ftv} 47$ & 6.77 & 0.02 & 2.39 & 0.28 & 36.04 & 6.54 & 0.11 & 1.84 & 1.83 & 58.27 & 1.54 & 37.43 & 1.52 & 256.06 & $\mathrm{tl}$ & 2.83 & 0.06 & 2.08 & 0.23 & 12.00 \\
\hline ftv55 & 10.55 & 0.03 & 3.89 & 0.27 & 43.74 & 10.31 & 0.16 & 4.97 & 1.19 & 91.16 & 1.49 & 87.38 & 1.43 & 324.61 & $\mathrm{tl}$ & 6.05 & 0.06 & 3.89 & 0.39 & 19.48 \\
\hline ftv64 & 6.31 & 0.05 & 4.21 & 0.28 & 114.50 & 5.84 & 0.22 & 4.00 & 1.98 & 322.56 & 1.71 & 182.06 & 1.63 & $1,368.27$ & $\mathrm{tl}$ & 4.24 & 0.06 & 4.03 & 0.67 & 32.53 \\
\hline ftv70 & 9.26 & 0.05 & 3.94 & 0.59 & 168.70 & 8.75 & 0.27 & 7.06 & 2.51 & $1,125.57$ & 2.10 & 285.50 & 1.99 & $\mathrm{tl}$ & tl & 4.69 & 0.06 & 4.05 & 0.87 & 52.40 \\
\hline $\mathrm{ft} 70$ & 1.77 & 0.06 & 1.16 & 0.64 & tl & 1.10 & 0.25 & 0.85 & 3.74 & 326.07 & 0.05 & 237.47 & 0.04 & $1,500.99$ & tl & 0.88 & 0.08 & 0.64 & 0.89 & 65.22 \\
\hline $\mathrm{ft} 53$ & 14.04 & 0.05 & 10.88 & 0.44 & 312.38 & 12.45 & 0.14 & 0.00 & 3.65 & 37.95 & 0.00 & 68.13 & 0.00 & 255.02 & 265.67 & 12.93 & 0.05 & 10.97 & 0.38 & tl \\
\hline \multirow[t]{2}{*}{ Avg } & 7.39 & 0.03 & 3.90 & 0.31 & 251.20 & 6.82 & 0.14 & 2.27 & 2.40 & 201.85 & 1.07 & 95.05 & 0.98 & 564.18 & $1,123.48$ & 4.69 & 0.05 & 3.91 & 0.41 & $\begin{array}{r}199.91 \\
(\mathrm{Q})\end{array}$ \\
\hline & \multicolumn{5}{|c|}{ GP } & \multicolumn{5}{|c|}{ SD } & \multicolumn{5}{|c|}{ SSB } & \multicolumn{5}{|c|}{ SST } \\
\hline Inst & $L P$ & $T_{L P}$ & $R t$ & $T_{R t}$ & $T_{I L P}$ & $L P$ & $T_{L P}$ & $R t$ & $T_{R t}$ & $T_{I L P}$ & $L P$ & $T_{L P}$ & $R t$ & $T_{R t}$ & $T_{I L P}$ & $L P$ & $T_{L P}$ & $R t$ & $T_{R t}$ & $T_{I L P}$ \\
\hline ftv33 & 0.00 & 0.85 & 0.00 & 1.97 & 8.21 & 4.78 & 0.25 & 4.53 & 0.61 & 26.91 & 0.00 & 0.41 & 0.00 & 0.71 & 0.77 & 0.00 & 277.58 & 0.00 & 496.90 & 496.90 \\
\hline $\mathrm{ftv} 35$ & 1.29 & 0.85 & 1.29 & 3.32 & 914.01 & 3.90 & 0.28 & 3.35 & 0.70 & 49.75 & 1.09 & 0.46 & 1.02 & 2.64 & 14.88 & 0.65 & 632.53 & 0.65 & $\mathrm{tl}$ & tl \\
\hline $\mathrm{ftv} 38$ & 1.24 & 1.32 & 1.24 & 5.38 & tl & 3.26 & 0.39 & 3.15 & 1.22 & 81.67 & 1.05 & 0.65 & 0.98 & 2.48 & 31.50 & 0.64 & 737.40 & 0.64 & $\mathrm{tl}$ & tl \\
\hline $\mathrm{ftv} 44$ & 1.84 & 1.58 & 1.26 & 8.24 & $\mathrm{tl}$ & 2.43 & 0.44 & 2.03 & 0.97 & 97.31 & 1.74 & 0.88 & 0.92 & 21.36 & 91.24 & - & - & - & - & - \\
\hline $\mathrm{ftv} 47$ & 1.86 & 1.63 & 1.53 & 6.27 & tl & 2.75 & 0.72 & 2.71 & 1.51 & 109.82 & 1.86 & 2.52 & 1.54 & 32.08 & $1,247.54$ & - & - & - & - & - \\
\hline ftv55 & 2.33 & 3.85 & 1.12 & 83.12 & tl & 5.89 & 0.53 & 5.62 & 4.18 & $1,105.22$ & 2.33 & 2.80 & 1.41 & 261.42 & 999.26 & - & - & - & - & - \\
\hline ftv64 & 3.30 & 6.64 & 2.99 & 189.60 & $\mathrm{tl}$ & 4.00 & 0.84 & 3.68 & 4.79 & 309.85 & 2.88 & 14.92 & 2.70 & 691.29 & tl & - & - & - & - & - \\
\hline ftv70 & 3.73 & 8.11 & 3.37 & 53.06 & tl & 4.64 & 2.22 & 4.45 & 6.99 & 525.08 & 3.12 & 27.54 & 2.63 & 476.80 & $\mathrm{tl}$ & - & - & - & - & - \\
\hline $\mathrm{ft} 70$ & 1.15 & 7.24 & 1.09 & 44.94 & tl & 0.80 & 1.36 & 0.74 & 7.46 & 236.81 & 0.55 & 14.66 & 0.48 & 38.21 & $\mathrm{tl}$ & - & - & - & - & - \\
\hline $\mathrm{ft} 53$ & 10.69 & 2.75 & 9.33 & 16.73 & tl & 11.39 & 0.48 & 11.26 & 1.47 & tl & 10.63 & 1.94 & 9.22 & 8.18 & tl & - & - & - & - & - \\
\hline Avg & 2.74 & 3.48 & 2.32 & 41.26 & $1,532.22$ & 4.38 & 0.75 & 4.15 & 2.99 & 434.24 & 2.53 & 6.68 & 2.09 & 153.51 & 958.52 & 0.43 & 549.17 & 0.43 & $1,365.63$ & $1,365.63$ \\
\hline & & & & & (2) & & & & & (9) & & & & & (6) & & & & & (1) \\
\hline
\end{tabular}


TABLE 2.3: Comparison between branch-and-bound algorithms, branch-and-cut algorithms and polynomial formulations

\begin{tabular}{|c|c|c|c|c|c|c|c|c|c|c|c|c|c|c|}
\hline \multirow[b]{2}{*}{ Inst } & \multicolumn{2}{|c|}{$C D T$} & \multicolumn{2}{|c|}{$F T$} & \multicolumn{2}{|c|}{$F L T$} & \multicolumn{2}{|c|}{ Concorde } & \multicolumn{2}{|c|}{$M T Z$} & \multicolumn{2}{|c|}{$G G$} & \multicolumn{2}{|c|}{$D L$} \\
\hline & $L B$ & $T$ & $L B$ & $T$ & $L B$ & $T$ & $L B$ & $T$ & $L B$ & $T$ & $L B$ & $T$ & $L B$ & $T$ \\
\hline br17 & 100.00 & 3.6 & 0.00 & 0.0 & 0.00 & 0.0 & 0.00 & 0.2 & 94.23 & 10.9 & 68.91 & 8.2 & 43.59 & 34.6 \\
\hline $\mathrm{ft} 53$ & 14.11 & $\mathrm{tl}$ & 1.56 & 0.2 & 0.00 & 0.1 & 0.00 & 0.6 & 14.04 & $3,123.8$ & 12.45 & 379.5 & 12.93 & $\mathrm{tl}$ \\
\hline $\mathrm{ft} 70$ & 1.80 & 0.4 & 0.57 & 0.3 & 0.02 & 0.2 & 0.01 & 3.2 & 1.77 & $\mathrm{tl}$ & 1.10 & $3,260.7$ & 0.88 & 652.2 \\
\hline $\mathrm{ftv} 33$ & 7.85 & 0.0 & 3.73 & 0.1 & 0.00 & 0.0 & 0.00 & 0.3 & 7.64 & 49.1 & 7.03 & 55.1 & 5.35 & 34.0 \\
\hline $\mathrm{ftv} 35$ & 6.25 & 0.0 & 3.53 & 0.2 & 0.88 & 0.4 & 0.68 & 9.0 & 6.12 & 62.7 & 5.59 & 119.3 & 4.04 & 52.3 \\
\hline ftv38 & 6.01 & 0.0 & 3.01 & 0.3 & 0.85 & 0.6 & 0.52 & 14.5 & 5.87 & 90.2 & 5.44 & 214.6 & 3.45 & 57.1 \\
\hline ftv 44 & 5.70 & 0.0 & 4.46 & 0.1 & 0.37 & 0.5 & 0.12 & 9.1 & 5.55 & 164.0 & 5.18 & 180.2 & 2.43 & 31.7 \\
\hline ftv 47 & 6.98 & 0.1 & 3.49 & 0.4 & 1.01 & 0.5 & 0.62 & 23.4 & 6.77 & 360.4 & 6.54 & 582.7 & 2.83 & 120.0 \\
\hline ftv55 & 10.76 & 1.1 & 6.59 & 1.5 & 0.81 & 1.4 & 0.44 & 9.0 & 10.55 & 437.4 & 10.31 & 911.6 & 6.05 & 194.8 \\
\hline ftv64 & 6.42 & 0.8 & 4.89 & 1.3 & 1.36 & 2.6 & 0.33 & 20.8 & 6.31 & $1,145.0$ & 5.84 & $3,225.6$ & 4.24 & 325.3 \\
\hline ftv70 & 9.44 & 3.3 & 6.92 & 3.7 & 0.92 & 1.1 & 0.26 & 17.8 & 9.26 & $1,687.0$ & 8.75 & $11,255.7$ & 4.69 & 524.0 \\
\hline ftv90 & 6.33 & 0.7 & 3.17 & 2.5 & 0.25 & 0.5 & 0.06 & 14.7 & 6.24 & 340.4 & 6.21 & tl & 1.72 & 498.1 \\
\hline $\mathrm{ftv} 100$ & 6.60 & 16.6 & 3.91 & 29.6 & 0.39 & 2.2 & 0.00 & 12.6 & 6.53 & $1,083.1$ & 6.49 & $\mathrm{tl}$ & 3.11 & $1,237.5$ \\
\hline $\mathrm{ftv} 110$ & 5.87 & 5.0 & 4.49 & 38.4 & 0.77 & 7.4 & 0.05 & 25.6 & 5.81 & $5,964.9$ & 5.71 & $\mathrm{tl}$ & 2.41 & $\mathrm{tl}$ \\
\hline $\mathrm{ftv} 120$ & 6.51 & 50.1 & 5.77 & 99.4 & 0.97 & 13.1 & 0.28 & 54.4 & 6.44 & $8,755.0$ & 6.32 & $\mathrm{tl}$ & 2.18 & tl \\
\hline $\mathrm{ftv} 130$ & 4.46 & 6.4 & 3.77 & 16.6 & 0.35 & 1.6 & 0.00 & 16.6 & 4.42 & $1,938.2$ & 4.34 & $\mathrm{tl}$ & 1.31 & 600.7 \\
\hline ftv140 & 4.92 & 13.9 & 4.26 & 38.8 & 0.25 & 2.1 & 0.00 & 25.6 & 4.87 & $1,127.6$ & 4.85 & $\mathrm{tl}$ & 1.41 & 287.3 \\
\hline ftv150 & 3.91 & 3.1 & 3.03 & 17.0 & 0.27 & 2.6 & 0.00 & 27.0 & 3.87 & 920.6 & 3.86 & tl & 1.31 & 402.7 \\
\hline ftv160 & 4.58 & 93.8 & 4.03 & 316.2 & 0.67 & 3.8 & 0.30 & 55.7 & 4.55 & $12,731.4$ & 4.54 & $\mathrm{tl}$ & 2.35 & $6,000.6$ \\
\hline ftv170 & 4.50 & $\mathrm{tl}$ & 4.14 & $\mathrm{tl}$ & 0.87 & 4.1 & 0.40 & 41.9 & 4.48 & tl & 4.47 & tl & 2.05 & $\mathrm{tl}$ \\
\hline kro124p & 6.22 & $\mathrm{tl}$ & 2.73 & 135.7 & 0.04 & 1.0 & 0.00 & 9.9 & 6.13 & $2,296.6$ & 5.47 & tl & 3.46 & $2,316.8$ \\
\hline $\mathrm{p} 43$ & 97.37 & tl & 0.37 & $\mathrm{tl}$ & 0.16 & 9.3 & 0.16 & 22.7 & 97.34 & $\mathrm{tl}$ & 85.23 & tl & 96.16 & $\mathrm{tl}$ \\
\hline rbg323 & 0.00 & 0.1 & 0.00 & 0.3 & 0.00 & 0.4 & 0.00 & 23.9 & 0.00 & $\mathrm{tl}$ & 0.00 & $\mathrm{tl}$ & 0.00 & $8,481.1$ \\
\hline rbg358 & 0.00 & 0.1 & 0.00 & 0.5 & 0.00 & 0.5 & 0.00 & 29.3 & 0.00 & $\mathrm{tl}$ & 0.00 & $\mathrm{tl}$ & 0.00 & $\mathrm{tl}$ \\
\hline rbg403 & 0.00 & 0.1 & 0.00 & 1.0 & 0.00 & 1.3 & 0.00 & 49.3 & 0.00 & $\mathrm{tl}$ & 0.00 & $\mathrm{tl}$ & 0.00 & tl \\
\hline rbg443 & 0.00 & 0.1 & 0.00 & 1.2 & 0.00 & 1.4 & 0.00 & 34.5 & 0.00 & tl & 0.00 & $\mathrm{tl}$ & 0.00 & $\mathrm{tl}$ \\
\hline ry48p & 13.21 & $\mathrm{tl}$ & 2.94 & 20.3 & 0.53 & 0.8 & 0.35 & 22.9 & 12.88 & $1,316.0$ & 11.17 & tl & 4.25 & $3,417.3$ \\
\hline balas84 & 14.07 & tl & 5.53 & 986.6 & 1.01 & 15.7 & 1.01 & 78.0 & 13.98 & $\mathrm{tl}$ & 12.34 & tl & 9.67 & $\mathrm{tl}$ \\
\hline balas108 & 25.00 & $\mathrm{tl}$ & 9.87 & $\mathrm{tl}$ & 1.97 & 89.0 & 2.63 & $1,416.0$ & 24.80 & $\mathrm{tl}$ & 18.14 & tl & 19.50 & tl \\
\hline balas120 & 21.68 & $\mathrm{tl}$ & 13.29 & $\mathrm{tl}$ & 1.05 & $1,276.3$ & 1.05 & $7,186.9$ & 21.43 & $\mathrm{tl}$ & 18.58 & tl & 16.86 & tl \\
\hline balas160 & 19.40 & $\mathrm{tl}$ & 11.34 & $\mathrm{tl}$ & 1.26 & 671.1 & 1.26 & $7,848.0$ & 19.18 & $\mathrm{tl}$ & 16.16 & tl & 18.83 & tl \\
\hline balas200 & 15.63 & $\mathrm{tl}$ & 8.68 & $\mathrm{tl}$ & 1.24 & $1,712.8$ & 0.74 & $2,294.2$ & 15.55 & $\mathrm{tl}$ & 12.86 & $\mathrm{tl}$ & 15.31 & tl \\
\hline Avg & 13.61 & $\begin{array}{l}9.06 \\
(22)\end{array}$ & 4.06 & $\begin{array}{r}65.85 \\
(26)\end{array}$ & 0.57 & $\begin{array}{r}119.51 \\
(32)\end{array}$ & 0.35 & $\begin{array}{r}606.18 \\
(32)\end{array}$ & \begin{tabular}{|l|l|}
13.33 \\
\end{tabular} & $\begin{array}{r}2,180.21 \\
(20)\end{array}$ & 11.37 & $\begin{array}{r}1,835.75 \\
(11)\end{array}$ & 9.14 & $\begin{array}{r}, 329.90 \\
(19)\end{array}$ \\
\hline $\begin{array}{l}\mathrm{Avg} \\
1,000 \mathrm{sec}\end{array}$ & - & $\begin{array}{r}318.7 \\
(22)\end{array}$ & - & $\begin{array}{r}241.0 \\
(26)\end{array}$ & - & $\begin{array}{l}88.6 \\
(30)\end{array}$ & - & $\begin{array}{r}145.4 \\
(28)\end{array}$ & - & $\begin{array}{r}794.9 \\
(9)\end{array}$ & - & $\begin{array}{r}826.6 \\
(8)\end{array}$ & - & $\begin{array}{r}681.7 \\
(14)\end{array}$ \\
\hline
\end{tabular}





\section{Chapter 3}

\section{Traveling Salesman Problem with Time Windows}

1

The traveling salesman problem with time windows (TSPTW) is the problem of finding, in a weighted digraph, a least-cost tour starting from a selected vertex, visiting each vertex of the graph exactly once within a given time window, and returning to the starting vertex. This chapter introduces new tour relaxations to compute valid lower bounds on the TSPTW. The optimal integer TSPTW solution is computed with a dynamic programming algorithm that uses bounding functions based on the different tour relaxations and the dual solutions obtained. An extensive computational analysis on basically all TSPTW instances (involving up to 233 vertices) from the literature is reported. The results show that the proposed algorithm solves all but one instances and outperforms all exact methods published in the literature so far.

\subsection{Introduction}

The traveling salesman problem with time windows (TSPTW) is defined on a digraph $G=\left(V^{\prime}, A\right)$, where $V^{\prime}$ is the vertex set and $A$ is the arc set. The vertex set $V^{\prime}$ is equal to $V \cup\{p, q\}$, where the set $V$ contains $n$ vertices $1,2, \ldots, n$, and vertices $p$ and $q$ are two special vertices. We indicate with $\Gamma_{i} \subseteq V^{\prime}$ the set of successors (i.e., $\left.\Gamma_{i}=\left\{j \in V^{\prime}:(i, j) \in A\right\}\right)$ and with $\Gamma_{i}^{-1} \subseteq V^{\prime}$ the set of predecessors (i.e., $\Gamma_{i}^{-1}=\{j \in$ $\left.\left.V^{\prime}:(j, i) \in A\right\}\right)$ of vertex $i \in V^{\prime}$ in graph $G$. We assume that $\Gamma_{p}^{-1}=\Gamma_{q}=\varnothing$ and $(p, q) \notin A$.

A time window $\left[e_{i}, l_{i}\right]$ is associated with each vertex $i \in V^{\prime}$, where $e_{i}$ and $l_{i}$ represent the earliest and latest time to visit vertex $i$, respectively. A travel cost $d_{i j}$ and a travel

\footnotetext{
${ }^{1}$ This chapter is based on Baldacci et al. [2011b]
} 
time $t_{i j}$ are associated with each arc $(i, j) \in A$; the travel time $t_{i j}$ includes the service time at vertex $i$. Travel times $t_{i j}$ and time windows $\left[e_{i}, l_{i}\right]$ are assumed integer values.

A salesman tour is a path in $G$ that starts from vertex $p$ at time $e_{p}$, visits each vertex $i \in V$ within its time window, and ends at vertex $q$ before time $l_{q}$. The salesman is allowed to arrive at a vertex $i \in V$ before time $e_{i}$, but, in this case, the service of vertex $i$ is postponed until time $e_{i}$. Hereafter, we assume that graph $G$ contains at least one salesman tour. The cost of a salesman tour is the sum of the travel costs of the arcs traversed. The TSPTW consists of finding a minimum-cost salesman tour.

The problem is $\mathcal{N} \mathcal{P}$-hard because it generalizes the classical traveling salesman problem (TSP). Even finding a feasible solution is $\mathcal{N} \mathcal{P}$-complete (see Savelsbergh [1985]).

The TSPTW has many practical applications, such as in single and multiple vehicle problems, control of stacker cranes in warehouses, bank deliveries, and postal deliveries.

\subsection{Literature Review}

The first exact algorithms are due to Christofides et al. [1981a] and Baker [1983]. They proposed branch-and-bound methods to solve a variant of the problem where the total schedule time must be minimized. The algorithm of Christofides et al. [1981a] was based on the technique introduced by Christofides et al. [1981c] called state-space relaxation (SSR), whereby the state-space associated with a given dynamic programming (DP) recursion is relaxed into a space of smaller cardinality in such a way that the solution of the relaxed recursion provides a lower bound to the original problem. Christofides et al. [1981a] and Baker [1983] reported solutions of TSPTW instances with up to 50 vertices.

Langevin et al. [1993] proposed a branch-and-bound algorithm based on a two-commodity flow formulation and reported solutions of instances with up to 60 vertices.

Algorithms based on DP were proposed by Dumas et al. [1995], Mingozzi et al. [1997], Balas and Simonetti [2001], and Li [2009]. Dumas et al. [1995] described a DP algorithm that applies sophisticated elimination tests to reduce the state-space and the number of state transitions; they reported the solutions of instances involving up to 200 vertices. Mingozzi et al. [1997] presented a DP algorithm for the TSP with time windows and precedence constraints based on the SSR technique and presented computational results for instances with up to 120 vertices. Balas and Simonetti [2001] considered a special case of the TSPTW where, for some initial ordering of the vertices, vertex $i$ precedes vertex $j$ if $j \geq i+k$ (for some value $k>0$ ), and described a DP algorithm that is linear in the number of vertices and exponential in $k$. Li [2009] presented a DP algorithm based on a bi-directional resource-bounded label correcting algorithm 
(see Righini and Salani [2006]); Li reported the solutions of instances involving up to 233 vertices and solved a number of open instances to optimality.

Branch-and-cut methods for the TSPTW were proposed by Ascheuer et al. [2001] and, recently, by Dash et al. [2012]. Ascheuer et al. [2001] described heuristic algorithms and branch-and-cut methods to solve a real-world application, called stacker crane optimization, that can be modeled as TSPTW. The cutting plane algorithms described by Ascheuer et al. [2001] use three alternative integer programming formulations of the TSPTW and are based on the formulations and polyhedral analysis presented in Ascheuer et al. [2000]. Ascheuer et al. [2001] reported computational results for realworld instances with up to 233 vertices, showing that most TSPTW instances with up to 70 vertices can be solved to optimality by any of the three models. Dash et al. [2012] introduced an extended formulation for the TSPTW based on partitioning the time windows into sub-windows, called buckets; they described a branch-and-cut algorithm that uses the valid inequalities described in Ascheuer et al. [2000, 2001] and new valid inequalities specific for the bucket formulation. Dash et al. reported the solutions of instances with up to 233 vertices and solved a number of open instances.

Focacci et al. [2002] proposed a hybrid approach for solving the TSPTW merging constraint programming algorithms and optimization techniques to compute bounds on the optimal solution value. Their algorithm solved instances with up to 69 vertices.

Heuristic algorithms were proposed, among others, by Gendreau et al. [1998], Wolfler Calvo [2000], Ohlmann and Thomas [2007], López Ibáñez and Blum [2010], and da Silva and Urrutia [2010].

\subsection{An Exact Algorithm for the TSPTW}

We assume that matrix $t_{i j}$ satisfies the triangle inequality, so the time windows and the arc set $A$ can be reduced by using the reduction rules described by Dash et al. [2012] and summarized in the following.

We denote by $\vec{G}=\left(V^{\prime}, \vec{A}\right)$ the precedence digraph, where arc $(i, j) \in \vec{A}$ indicates that vertex $i \in V^{\prime}$ must precede vertex $j \in V^{\prime}$ in any salesman tour. The arc set $\vec{A}$ is obtained by (i) setting $\vec{A}=\Gamma_{p} \cup \Gamma_{q}^{-1}$ and adding $\operatorname{arc}(i, j) \in A$ to $\vec{A}$ for any couple of vertices $i, j \in V$ such that $e_{j}+t_{j i}>l_{i}$; (ii) adding, to $\vec{A}$, any $\operatorname{arc}(i, j) \in A$ if there exists a vertex $k \in V \backslash\{i, j\}$ such that both $\operatorname{arcs}(i, k)$ and $(k, j)$ belong to $\vec{A}$. The latter operation is repeated until no more arcs are added to $\vec{A}$. Then, for any arc $(i, j) \in \vec{A}$, the arc $(j, i)$ is removed from the arc set $A$ of graph $G$ because it cannot belong to any salesman tour.

We indicate with $\vec{\Gamma}_{i} \subseteq V^{\prime}, i \in V^{\prime}$, the set of successors and with $\vec{\Gamma}_{i}^{-1} \subseteq V^{\prime}, i \in V^{\prime}$, the set of predecessors of vertex $i \in V^{\prime}$ in graph $\vec{G}$. With each $\operatorname{arc}(i, j) \in A$, we associate 
a time $\vec{t}_{i j}$, which represents a lower bound on the minimum time spent between the visits to vertices $i$ and $j$ in any salesman tour, computed as the cost of the longest path in $\vec{G}$ from vertex $i$ to vertex $j$ using travel time $t_{i j}$ as cost of $\operatorname{arc}(i, j) \in \vec{A}$. We assume $\vec{t}_{i i}=0, i \in V$.

A forward path $F=\left(p, i_{1}, \ldots, i_{k}=\sigma_{F}\right)$ is an elementary path that starts from vertex $p$ at time $e_{p}$, visits vertices $V(F)=\left\{p, i_{1}, \ldots, i_{k}\right\}$ within their time windows, and ends at vertex $\sigma_{F}$ at time $t_{F} \in\left[e_{\sigma_{F}}, l_{\sigma_{F}}\right]$. The cost, $c(F)$, of a forward path $F$ is the sum of the costs of the traversed arcs. Let $f(S, t, i)$ be the cost of a least-cost forward path $F$ that visits the set of vertices $S \subseteq V^{\prime}$ and ends at vertex $i \in S$ at time $t \in\left[e_{i}, l_{i}\right]$. The $\operatorname{cost} z^{*}$ of a TSPTW optimal solution is given by

$$
z^{*}=\min _{t \in\left[e_{q}, l_{q}\right]}\left\{f\left(V^{\prime}, t, q\right)\right\}
$$

Let $\Omega(t, j, i), j, i \in V^{\prime}, t \in\left[e_{i}, l_{i}\right]$, be the set of departure times from vertex $j$ to arrive at vertex $i$ at time $t$ if $j$ is visited immediately before $i$. The set $\Omega(t, j, i)$ is defined as

$$
\Omega(t, j, i)= \begin{cases}\left.t^{\prime}: t^{\prime} \in\left[e_{j}, \min \left\{l_{j}, t-t_{j i}\right\}\right]\right\}, & \text { if } t=e_{i}, \\ t-t_{j i}: t-t_{j i} \in\left[e_{j}, l_{j}\right], & \text { if } t \in\left(e_{i}, l_{i}\right] .\end{cases}
$$

Functions $f(S, t, i)$ can be computed with DP on a graph $\mathscr{G}_{F}=\left(\mathscr{F}, \mathscr{A}_{F}\right)$ as follows. The vertex set $\mathscr{F}$ represents the states of the DP recursion and is defined as

$$
\mathscr{F}=\left\{(S, t, i): S \subseteq V^{\prime}, i \in S, t \in\left[e_{i}, l_{i}\right]\right\} .
$$

The arc set $\mathscr{A}_{F}$ represents the transitions of the DP recursion and is defined as $\mathscr{A}_{F}=\left\{\left(\left(S^{\prime}, t^{\prime}, j\right),(S, t, i)\right):\left(S^{\prime}, t^{\prime}, j\right),(S, t, i) \in \mathscr{F}, S^{\prime}=S \backslash\{i\}, t^{\prime} \in \Omega(t, j, i), j \in \Gamma_{i}^{-1} \cap S\right\}$.

A possible DP recursion for computing functions $f(S, t, i)$ is

$$
f(S, t, i)=\min _{\left(S^{\prime}, t^{\prime}, j\right) \in \mathscr{F}:\left(\left(S^{\prime}, t^{\prime}, j\right),(S, t, i)\right) \in \mathscr{A}_{F}}\left\{f\left(S^{\prime}, t^{\prime}, j\right)+d_{j i}\right\}, \quad(S, t, i) \in \mathscr{F} .
$$

It is required to initialize $f\left(\{p\}, e_{p}, p\right)=0$ and $f(\{p\}, t, p)=\infty$, for each $t \in\left(e_{p}, l_{p}\right]$.

The size of the path set $\mathscr{F}$ can be reduced by removing any state $(S, t, i) \in \mathscr{F}$ that cannot lead to any optimal TSPTW solution according to the following rules.

Dominance 1. Let $(S, t, i),\left(S, t^{\prime}, i\right) \in \mathscr{F}$ be two states such that $f(S, t, i) \geq f\left(S, t^{\prime}, i\right)$ and $t>t^{\prime}$, then $(S, t, i)$ is dominated by $\left(S, t^{\prime}, i\right)$.

Fathoming 1. Any state $(S, t, i) \in \mathscr{F}$ cannot lead to any feasible solution if (i) there exists a vertex $j \in V^{\prime} \backslash S$ such that $t+\vec{t}_{i j}>l_{j}$ or (ii) $\vec{\Gamma}_{i}^{-1} \nsubseteq \subseteq S$.

Fathoming 2. Let $z_{U B}$ be an upper bound on the TSPTW, and let $b(S, t, i)$ be a lower bound on the cost of a least-cost path that starts from vertex $i \in S$ at time $t \in\left[e_{i}, l_{i}\right]$, visits each vertex in $V^{\prime} \backslash S$ within its time window, and ends at vertex $q$ before time 
$l_{q}$. Any state $(S, t, i)$ such that $f(S, t, i)+b(S, t, i) \geq z_{U B}$ cannot lead to any optimal solution.

In $\S 3.6$, we describe three methods for computing bounding functions $b(S, t, i)$ based on three SSRs of recursion (3.1) and on a valid lower bound $L B$ described in $\S 3.5$.

To avoid the a priori computation of an upper bound $z_{U B}$, we use an iterative algorithm where, at iteration $h$, recursion (3.1) is computed and Fathoming 2 is applied using a not necessarily valid upper bound $z_{U B}^{h}$. The exact algorithm can be outlined as follows.

Step 1. Compute a lower bound $L B$ as described in $\S 3.5$, and set $z_{U B}^{0}=L B$. Initialize $h=1, \theta=\max \left\{\left\lceil 10^{-3} L B\right\rceil, 1\right\}$, and $z_{U B}^{1}=\lceil L B\rceil+\theta$.

Step 2. Compute DP recursion (3.1) by using the bounding functions $b(S, t, i)$ described in $\S 3.6$ and $z_{U B}^{h}$ instead of $z_{U B}$ in Fathoming 2.

Step 3. There are two possible cases.

i) No state $\left(V^{\prime}, t, q\right)$, with $t \in\left[e_{q}, l_{q}\right]$, was generated (this happens if $z^{*}>$ $\left.z_{U B}^{h}\right)$. Set $h=h+1, z_{U B}^{h}=z_{U B}^{h-1}+\theta$, and go to Step 2.

ii) At least a state $\left(V^{\prime}, t, q\right)$, with $t \in\left[e_{q}, l_{q}\right]$, was generated. The optimal TSPTW solution cost is $z^{*}=\min _{t \in\left[e_{q}, l_{q}\right]}\left\{f\left(V^{\prime}, t, q\right)\right\}$.

The algorithm can terminate prematurely at iteration $h$, while computing recursion (3.1) at Step 2, if the number of generated states exceeds the computer memory available. In this case, $z_{U B}^{h-1}$ represents a valid lower bound on the TSPTW.

\subsection{Relaxations of the TSPTW}

We describe three relaxations of salesman tours, called $t$-tour, $n g$-tour and $n g L$-tour relaxations, that are used to compute valid lower bounds on the TSPTW and bounding functions $b(S, t, i)$ used by Fathoming 2 .

\subsubsection{The $t$-Tour Relaxation}

The $t$-tour relaxation was introduced by Christofides et al. [1981c]. This relaxation provides function $f(t, i)$ that corresponds to the cost of a least-cost nonnecessarily elementary path, called $(t, i)$-path, that starts from vertex $p$ at time $e_{p}$, visits a set of vertices (without two-vertex loops) within their time windows, and ends at vertex $i \in V^{\prime}$ at time $t \in\left[e_{i}, l_{i}\right]$. The DP recursion for computing functions $f(t, i)$ is described in Christofides et al. A $t$-tour is the $\left(t^{*}, q\right)$-path such that $f\left(t^{*}, q\right)=\min _{t \in\left[e_{q}, l_{q}\right]}\{f(t, q)\}$. 


\subsubsection{The $n g$-Tour Relaxation}

In general, the $t$-tour relaxation does not provide any detailed knowledge of the path of cost $f(t, i)$, so additional conditions to ensure that such path provides a feasible solution to the original problem cannot be imposed. To alleviate this drawback, we introduce the $n g$-path relaxation that consists of partitioning the set of all possible $(t, i)$-paths ending at vertex $i \in V^{\prime}$ at time $t \in\left[e_{i}, l_{i}\right]$ according to a mapping function that associates with each $(t, i)$-path a subset of the visited vertices depending on the order in which they are visited. The subset of vertices associated with each $n g$-path is used in the DP recursion to impose partial elementarity. The $n g$-tour relaxation can be described as follows.

Let $N_{i} \subseteq V^{\prime}$ be a set of selected vertices for vertex $i \in V^{\prime}$ (according to some criterion) such that $i \in N_{i}$ and $\left|N_{i}\right| \leq \Delta\left(N_{i}\right)$, where $\Delta\left(N_{i}\right)$ is a parameter. The sets $N_{i}$ allow us to associate, with each forward path $F=\left(p, i_{1}, \ldots, i_{k}=\sigma_{F}\right)$, the set $\Pi_{F} \subseteq V(F)$ containing vertex $i_{k}$ and every vertex $i_{r} \in V(F), r=1, . ., k-1$, that belongs to all sets $N_{i_{r+1}}, \ldots, N_{i_{k}}$ associated with the vertices $i_{r+1}, \ldots, i_{k}$ visited after $i_{r}$ in path $F$. Formally, the set $\Pi_{F}$ is defined as

$$
\Pi_{F}=\left\{i_{r} \in V(F) \backslash\left\{i_{k}\right\}: i_{r} \in \bigcap_{s=r+1}^{k} N_{i_{s}}\right\} \bigcup\left\{i_{k}\right\} .
$$

A forward $n g$-path $(N G, t, i)$ is a nonnecessarily elementary path $F=\left(p, i_{1}, \ldots, i_{k-1}\right.$, $i_{k}=i$ ) that starts from vertex $p$ at time $e_{p}$, visits a set of vertices (each once or more) within their time windows, ends at vertex $i \in V^{\prime}$ at time $t \in\left[e_{i}, l_{i}\right]$, and such that $N G=\Pi_{F}$ and $i \notin \Pi_{F^{\prime}}$, where $F^{\prime}=\left(p, i_{1}, \ldots, i_{k-1}\right)$ is an $n g$-path.

Notice that an $n g$-path can contain a loop $\left(i_{r}=j, i_{r+1}, \ldots, i_{r+s}=j\right)$ for $s \geq 2$ if and only if there exists at least one index $k$ such that $2 \leq k \leq s-1$ and $j \notin N_{i_{r+k}}$.

An example of $n g$-path is the following. Consider path $F=(p, 1,2,3,4,1)$. The corresponding path $F^{\prime}$ is $F^{\prime}=(p, 1,2,3,4)$. Suppose that sets $N_{i}, i=1,2,3,4$, are defined as $N_{1}=\{1,3,4\}, N_{2}=\{1,2,5\}, N_{3}=\{1,3,4\}, N_{4}=\{2,3,4\}$. The set $\Pi_{F^{\prime}}$ contains vertices 3 and 4 only because $1 \notin N_{2} \cap N_{3} \cap N_{4}$ and $2 \notin N_{3} \cap N_{4}$ whereas $3 \in N_{4}$ and 4 is the last vertex visited by path $F^{\prime}$. Being elementary, path $F^{\prime}$ is clearly an $n g$-path. Thus, because $1 \notin \Pi_{F^{\prime}}$, path $F$ is an $n g$-path. Moreover, the set $\Pi_{F}$ contains vertices 1,3 and 4 only because $2 \notin N_{3} \cap N_{4} \cap N_{1}$ whereas $3 \in N_{4} \cap N_{1}$, $4 \in N_{1}$ and 1 is the last vertex visited by path $F$; this means that path $F$ can be propagated towards all vertices but 1,3 and 4 .

We denote by $f(N G, t, i)$ the cost of a least-cost forward $n g$-path $(N G, t, i)$. Any $(N G, t, q)$-path ending at vertex $q$ at time $t \in\left[e_{q}, l_{q}\right]$ is called $n g$-tour. 
A valid lower bound $L B$ on the TSPTW is given by

$$
L B=\min _{t \in\left[e_{q}, l_{q}\right], N G \subseteq N_{q}}\{f(N G, t, q)\} \leq z^{*} .
$$

Functions $f(N G, t, i)$ can be computed with DP on a graph $\widehat{\mathscr{G}_{F}}=\left(\widehat{\mathscr{F}}, \widehat{\mathscr{A}_{F}}\right)$. The vertex set $\widehat{\mathscr{F}}$ represents the states of the DP recursion and is defined as

$$
\widehat{\mathscr{F}}=\left\{(N G, t, i): i \in V^{\prime}, t \in\left[e_{i}, l_{i}\right], \forall N G \subseteq N_{i} \text { s.t. } N G \ni i\right\} .
$$

The arc set $\widehat{\mathscr{A}_{F}}$ represents the transitions of the DP recursion and is defined as

$$
\begin{gathered}
\widehat{\mathscr{A}_{F}}=\left\{\left(\left(N G^{\prime}, t^{\prime}, j\right),(N G, t, i)\right):\left(N G^{\prime}, t^{\prime}, j\right),(N G, t, i) \in \widehat{\mathscr{F}},\right. \\
\left.j \in \Gamma_{i}^{-1}, t^{\prime} \in \Omega(t, j, i), \forall N G^{\prime} \subseteq N_{j} \text { s.t. } N G^{\prime} \ni j \text { and } N G^{\prime} \cap N_{i}=N G \backslash\{i\}\right\} .
\end{gathered}
$$

A possible DP recursion for computing functions $f(N G, t, i)$ is

$$
f(N G, t, i)=\min _{\left(N G^{\prime}, t^{\prime}, j\right) \in \widehat{\mathscr{F}}:\left(\left(N G^{\prime}, t^{\prime}, j\right),(N G, t, i)\right) \in \widehat{\mathscr{A}_{F}}}\left\{f\left(N G^{\prime}, t^{\prime}, j\right)+d_{j i}\right\}, \quad(N G, t, i) \in \widehat{\mathscr{F}} .
$$

Notice that the condition $N G^{\prime} \cap N_{i}=N G \backslash\{i\}$ in the definition of $\widehat{\mathscr{A}_{F}}$ imposes that functions $f(N G, t, i)$ are computed by propagating functions $f\left(N G^{\prime}, t^{\prime}, j\right)$ such that $i \notin N G^{\prime}$. Moreover, notice that the following inequality holds for each forward path $F$

$$
c(F) \geq \min _{N G \subseteq V(F) \cap N_{\sigma_{F}}}\left\{f\left(N G, t_{F}, \sigma_{F}\right)\right\} .
$$

It is required to initialize $f\left(\{p\}, e_{p}, p\right)=0$ and $f(\{p\}, t, p)=\infty$, for each $t \in\left(e_{p}, l_{p}\right]$.

An optimal $n g$-tour is the $n g$-path $\left(N G^{*}, t^{*}, q\right)$ such that

$$
f\left(N G^{*}, t^{*}, q\right)=\min _{(N G, t, q) \in \widehat{\mathscr{F}}}\{f(N G, t, q)\}
$$

The size of the state set $\widehat{\mathscr{F}}$ can be reduced by removing, via the following dominance rule, any state that cannot lead to an optimal $n g$-tour.

Dominance 2. Let $(N G, t, i),\left(N G^{\prime}, t^{\prime}, i\right) \in \widehat{\mathscr{F}}$ such that $f(N G, t, i) \geq f\left(N G^{\prime}, t^{\prime}, i\right)$, $N G \supseteq N G^{\prime}, t>t^{\prime}$. State $(N G, t, i)$ is dominated by $\left(N G^{\prime}, t^{\prime}, i\right)$.

Notice that a stronger version of Dominance 2 can be obtained by replacing the condition $t<t^{\prime}$ with $t \leq t^{\prime}$. However, we found it to be computationally convenient to apply Dominance 2 instead of this stronger version.

The decremental state-space relaxation introduced by Righini and Salani [2008] and the partial elementarity relaxation introduced by Desaulniers et al. [2008] are special 
cases of the $n g$-path relaxation. These relaxations can be obtained by setting $N_{i}=\widehat{V}$, for each vertex $i \in V^{\prime}$, where $\widehat{V} \subseteq V^{\prime}$ is a selected set of vertices.

Choosing the sets $\boldsymbol{N}_{\boldsymbol{i}}$ of selected vertices. Lower bound $L B$ computed by expression (3.2) depends on the sets $N_{i}, i \in V^{\prime}$. If $N_{i}=V^{\prime}$, for each vertex $i \in V^{\prime}$, functions $f(N G, t, i)$ provide the costs of least-cost elementary paths and the optimal $n g$-tour provided by expression (3.4) corresponds to an optimal TSPTW solution.

The computational results of $\S 3.7$ were obtained by defining the sets $N_{i}, i \in V^{\prime}$, as follows. We set $N_{p}=\{p\}$ and $N_{q}=\{q\}$, and define $T_{i}=\left\{j \in \Gamma_{i} \cap \Gamma_{i}^{-1}: t_{i j}=\right.$ 0 or $\left.t_{j i}=0\right\}$, for each vertex $i \in V$ (notice that the set $T_{i}$ contains vertex $i$ ). First, we set $N_{i}=T_{i}$, for each vertex $i \in V$. Then, for each vertex $i \in V$ such that $\left|N_{i}\right|<\Delta\left(N_{i}\right)$, we add, to $N_{i}$, the $\left(\Delta\left(N_{i}\right)-\left|T_{i}\right|\right)$-nearest vertices to $i$ belonging to the set $V \backslash T_{i}$ according to travel times $t_{i j}$.

Notice that recursion (3.3) allows $n g$-paths to contain two-vertex loops, which can be eliminated with the method described by Christofides et al. [1981c]. Nonetheless, we do not implement this method because of the additional memory required and because, in practice, whenever sets $N_{i}, i \in V^{\prime}$, are defined as above and parameter $\Delta\left(N_{i}\right)$ is equal to 11 or 13 , the resulting $(N G, t, i)$-paths rarely contain two-vertex loops.

Implementation issues. How the DP recursion (3.3) is computed and how Dominance 2 is tested affect the effectiveness of the whole solution process. We decided to compute (3.3) with a forward DP recursion using the variable state $t$ (i.e., the time) as stage. At stage $t$, the order in which states are propagated is defined by a queue where states having time $t$ are maintained.

To apply Dominance 2, we use an additional function $F_{t}(N G, i), i \in V, N G \subseteq$ $N_{i}$ s.t. $N G \ni i$, that represents the cost of a least-cost state $\left(N G, t^{\prime}, i\right) \in \widehat{\mathscr{F}}$ with $t^{\prime}<t$ (i.e., $\left.F_{t}(N G, i)=\min _{t^{\prime} \in\left[e_{i}, t\right)}\left\{f\left(N G, t^{\prime}, i\right):\left(N G, t^{\prime}, i\right) \in \widehat{\mathscr{F}}\right\}\right)$. Let $\mathscr{B}(N G, i)=$ $\left\{N G^{\prime} \subseteq N G: N G^{\prime} \ni i\right\}$, for each vertex $i \in V$ and each set $N G \subseteq N_{i}$ s.t. $N G \ni i$. Because we use values of parameter $\Delta\left(N_{i}\right)$ less than or equal to 13 , the sets $\mathscr{B}(N G, i)$ can be computed by complete enumeration before starting the DP recursion. From the definitions of $F_{t}(N G, i)$ and $\mathscr{B}(N G, i)$, we derive that a state $(N G, t, i)$ is dominated according to Dominance 2 if $f(N G, t, i) \geq \min _{N G^{\prime} \in \mathscr{B}(N G, i)}\left\{F_{t}\left(N G^{\prime}, i\right)\right\}$. Notice that function $F_{t}(N G, i)$ can be recursively computed as

$$
F_{t}(N G, i)=\min \left\{F_{t-1}(N G, i), f(N G, t-1, i)\right\}, \quad i \in V, N G \subseteq N_{i} \text { s.t. } N G \ni i .
$$

\subsubsection{The $n g L$-Tour Relaxation}

In the case of the TSPTW, the $n g$-tour relaxation can be enhanced by forcing any $n g$ tour to visit each vertex of a selected subset of vertices exactly once while satisfying the precedence constraints imposed for such vertices by the precedence digraph $\vec{G}$. 
Consider a path $L=\left(p=i_{0}, i_{1}, \ldots, i_{h}=q\right)$ in $\vec{G}$ from vertex $p$ to vertex $q$. Because any $\operatorname{arc}(i, j) \in \vec{A}$ of graph $\vec{G}$ implies that vertex $i$ must be visited before vertex $j$ in any salesman tour, the $h$ arcs of path $L$ decompose any salesman tour in $h$ subpaths $P_{i_{k-1} i_{k}}, k=1, \ldots, h$, where $P_{i_{k-1} i_{k}}$ corresponds to the subpath of the salesman tour that starts from vertex $i_{k-1}$, visits each vertex of subset $S_{k} \subseteq V^{\prime} \backslash V(L)$ exactly once, and ends at vertex $i_{k}$. Thus, any salesman tour is made up of any collection of $h$ subpaths $P_{i_{k-1} i_{k}}, k=1, \ldots, h$, such that $\cup_{k=1}^{h} S_{k}=V^{\prime} \backslash V(L)$. For a given path $L$ of $\vec{G}$ defined as above, we can force any $n g$-tour to visit, exactly once, each vertex $i \in V(L)$ as described in the following.

Let $V_{k}$ be the set of vertices that must or can be visited in between vertices $i_{k-1}$ and $i_{k}\left(i_{k-1}, i_{k} \in L\right)$. For each $k=1, \ldots, h$, we have

$$
V_{k}=\left\{j \in V \backslash\left\{i_{k-1}\right\}: e_{i_{k-1}}+\vec{t}_{i_{k-1} j} \leq l_{j} \text { and } \max \left\{e_{i_{k-1}}+\vec{t}_{i_{k-1} j}, e_{j}\right\}+\vec{t}_{j i_{k}} \leq l_{i_{k}}\right\} .
$$

Notice that $V_{k} \cap V(L)=i_{k}$.

A forward $n g L(k)$-path is an $n g$-path $(N G, t, i)$ that ends at vertex $i \in V_{k}$ at time $t \in\left[e_{i}, l_{i}\right]$, and such that each vertex $j \in\left\{i_{0}, i_{1}, \ldots, i_{k-1}\right\}$ is visited exactly once. An $n g L$-tour is an $n g L(h)$-path $(N G, t, q)$.

Let $\widehat{\mathscr{F}}_{k}=\left\{(N G, t, i) \in \widehat{\mathscr{F}}: i \in V_{k}\right\}$ be the subset of states corresponding to the forward $n g L(k)$-paths, and let $f_{k}(N G, t, i)$ be the cost of a least-cost $n g L(k)$-path $(N G, t, i) \in \widehat{\mathscr{F}}_{k}$.

Functions $f_{k}(N G, t, i), k=1, \ldots, h, i \in V^{\prime}, N G \subseteq N_{i}$ s.t. $N G \ni i, t \in\left[e_{i}, l_{i}\right]$, can be computed with the following iterative procedure that, for each $k=1, \ldots, h$, performs the following operations

a) Initialize $f_{k}\left(N G, t, i_{k-1}\right)=f_{k-1}\left(N G, t, i_{k-1}\right)$, for each state $\left(N G, t, i_{k-1}\right) \in \widehat{\mathscr{F}}_{k-1}$, and $f_{k}(N G, t, i)=\infty$, for each state $(N G, t, i) \in \widehat{\mathscr{F}}_{k}$. We assume $f_{0}\left(\{p\}, e_{p}, p\right)=0$ and $f_{0}(\{p\}, t, p)=\infty$, for each $t \in\left(e_{p}, l_{p}\right]$;

b) Compute $f_{k}(N G, t, i),(N G, t, i) \in \widehat{\mathscr{F}}_{k}$, as follows

$$
f_{k}(N G, t, i)=\min _{\substack{\left(N G^{\prime}, t^{\prime}, j\right) \in \widehat{\mathscr{F}}_{k}: j \in \Gamma_{i}^{-1}, t^{\prime} \in \Omega(t, j, i), N G^{\prime} \subseteq N_{j} \text { s.t. } N G^{\prime} \ni j, N G^{\prime} \cap N_{i}=N G \backslash\{i\}}}\left\{f_{k}\left(N G^{\prime}, t^{\prime}, j\right)+d_{j i}\right\} .
$$

Because of the initialization and of the definition of the sets $V_{k}, k=1, \ldots, h$, any $n g L(h)$-path corresponding to $f_{h}(N G, t, q)$ visits every vertex of the set $V(L)$ exactly once.

An optimal $n g L$-tour is the $n g L(h)$-path $\left(N G^{*}, t^{*}, q\right) \in \widehat{\mathscr{F}}_{h}$ such that

$$
f_{h}\left(N G^{*}, t^{*}, q\right)=\min _{(N G, t, i) \in \widehat{\mathscr{F}}_{h}}\left\{f_{h}(N G, t, i)\right\}
$$


The value $f_{h}\left(N G^{*}, t^{*}, q^{*}\right)$ depends on the path $L$ of $\vec{G}$ used. In our computational experiments, the path $L$ was chosen as the path in $\vec{G}$ from vertex $p$ to vertex $q$ of maximal cardinality in order to maximize the number of vertices visited exactly once by any $n g L$-tour.

The following dominance rule, similar to Dominance 2 described in $\S 3.4 .2$ for the $n g$ tour relaxation, can be used to reduce the size of each state sets $\widehat{\mathscr{F}}_{k}, k=1, \ldots, h$.

Dominance 3. Let $(N G, t, i),\left(N G^{\prime}, t^{\prime}, i\right) \in \widehat{\mathscr{F}}_{k}$ be such that $f_{k}(N G, t, i) \geq f_{k}\left(N G^{\prime}, t^{\prime}, i\right)$, $N G^{\prime} \supseteq N G, t>t^{\prime}$. State $(N G, t, i)$ is dominated by $\left(N G^{\prime}, t^{\prime}, i\right)$ and can be removed from $\widehat{\mathscr{F}}_{k}$.

\subsection{A Valid Lower Bound $L B$ on the TSPTW}

In this section, we describe lower bounds on the TSPTW based on the three tour relaxations described in $\S 3.4$.

Let $\widehat{\mathscr{H}}$ be the index set of the tours of a given tour relaxation of $G$ (i.e., $t$-tour or $n g$-tour or $n g L$-tour). For each tour $\ell \in \widehat{\mathscr{H}}$, we denote by $a_{i \ell}$ the number of times vertex $i \in V$ is visited by tour $\ell \in \widehat{\mathscr{H}}$ and by $c_{\ell}$ its cost. Associate a penalty $u_{i} \in \mathbb{R}$ with each vertex $i \in V$, and let $\boldsymbol{u}=\left(u_{1}, \ldots, u_{n}\right)$. For a given penalty vector $\boldsymbol{u}$, a valid lower bound $L R(\boldsymbol{u})$ on the TSPTW is given by

$$
L R(\boldsymbol{u})=\min _{\ell \in \mathscr{\mathscr { H }}}\left\{c_{\ell}-\sum_{i \in V} a_{i \ell} u_{i}\right\}+\sum_{i \in V} u_{i} .
$$

A better lower bound $L B$ on the TSPTW can be computed by using subgradient optimization to solve the following Lagrangean dual problem $L D$

$$
(L D) \quad z(L D)=\max _{\boldsymbol{u} \in \mathbb{R}^{n}}\{L R(\boldsymbol{u})\}
$$

Solving problem $L D$ by usual subgradient optimization requires to find, at each iteration, an optimal value of problem $L R(\boldsymbol{u})$ for a given penalty vector $\boldsymbol{u}$. The optimal value of problem $L R(\boldsymbol{u})$ is computed with the DP recursion associated with the tour relaxation chosen by replacing arc costs $d_{i j}$ with $d_{i j}^{\prime}=d_{i j}-u_{j}$, for each arc $(i, j) \in A \backslash A_{q}$, and $d_{i q}^{\prime}=d_{i q}$, for each arc $(i, q) \in A_{q}$, where $A_{q}=\left\{(i, q): i \in \Gamma_{q}^{-1}\right\}$. 
Problem $L D$ is equivalent to the following linear program $D$

$$
\begin{aligned}
& \text { (D) } z(D)=\max u_{0}+\sum_{i \in V} u_{i} \text {, } \\
& \text { s.t. } u_{0} \leq c_{\ell}-\sum_{i \in V} a_{i \ell} u_{i}, \quad \ell \in \widehat{\mathscr{H}}, \\
& u_{0} \in \mathbb{R} \text {, } \\
& u_{i} \in \mathbb{R}, \quad i \in V \text {. }
\end{aligned}
$$

Dualizing $D$, we obtain the following problem $P$

$$
\begin{aligned}
(P) \quad z(P)=\min & \sum_{\ell \in \widehat{\mathscr{H}}} c_{\ell} x_{\ell}, \\
\text { s.t. } & \sum_{\ell \in \widehat{\mathscr{H}}} a_{i \ell} x_{\ell}=1, \quad i \in V, \\
& \sum_{\ell \in \widehat{\mathscr{H}}} x_{\ell}=1, \\
& x_{\ell} \geq 0, \quad \ell \in \widehat{\mathscr{H}} .
\end{aligned}
$$

Problem $P$ seeks a minimum-weight convex combination of tours such that each vertex $i \in V$ has, on average, degree one. Notice that variables $u_{i} \in \mathbb{R}, i \in V$, and $u_{0} \in \mathbb{R}$ of problem $D$ represent the dual variables of constraints (3.11) and (3.12), respectively.

Problem $P$ can be solved by column generation where, at each iteration, tours of negative reduced cost with respect to the dual solution of the current master problem must be computed. In our computational experiments, we found that this method of solving $P$ requires fewer iterations than solving $L D$ with subgradient optimization.

To solve $P$, we use a column generation procedure that differs from standard column generation methods because the master problem is not solved with the simplex but using a dual-ascent heuristic procedure. The procedure computes lower bound $L B_{1}$ corresponding to a $P$ dual solution $\boldsymbol{u}^{\mathbf{1}}=\left(u_{0}^{1}, u_{1}^{1}, \ldots, u_{n}^{1}\right)$.

Below we describe how we initialize the master problem, the method for solving the master problem, and the procedure for solving the pricing problem.

\subsubsection{Initializing the Master Problem}

The initial master problem contains a small subset of elementary but nonnecessarily Hamiltonian tours that are generated as follows. The procedure consists of executing a limited number of subgradient iterations to solve problem $L D$. The optimal tour found at each iteration is made elementary and then added to the master. At the end, the procedure provides a lower bound $L B_{0}$ and a corresponding solution of problem $L D$. The procedure can be described as follows. 
- Set $L B_{0}=0$. Initialize the Lagrangean penalties $\mu_{i} \in \mathbb{R}, i \in V$, as $\mu_{i}=0$.

- Perform Maxit0 iterations of the following subgradient method.

a) Define $d_{i j}^{\prime}=d_{i j}-\mu_{j}$, for each arc $(i, j) \in A \backslash A_{q}$, and $d_{i q}^{\prime}=d_{i q}$, for each arc $(i, q) \in A_{q}$.

b) Find the tour $\ell^{*} \in \widehat{\mathscr{H}}$ (i.e., $t$-tour or $n g$-tour or $n g L$-tour) of minimum cost, $c_{\ell^{*}}^{\prime}$, with respect to the modified arc costs $d_{i j}^{\prime}$.

c) If $c_{\ell^{*}}^{\prime}+\sum_{i \in V} \mu_{i}>L B_{0}$, update $L B_{0}=c_{\ell^{*}}^{\prime}+\sum_{i \in V} \mu_{i}$, and set $\mu_{i}^{0}=\mu_{i}$, for each vertex $i \in V$.

d) Add, to the master problem, the elementary but nonnecessarily Hamiltonian tour derived, from tour of index $\ell^{*}$, by removing the visits to the vertices that have already been visited in the tour.

e) Because in any salesman tour each vertex $i \in V$ must be visited exactly once, update penalties $\mu_{i}, i \in V$, using usual subgradient expressions setting $z_{U B}=1.2\left(c_{\ell^{*}}^{\prime}+\sum_{i \in V} \mu_{i}\right)$ in computing the step size.

Notice that $\boldsymbol{u}^{0}=\left(u_{0}^{0}, u_{1}^{0}, \ldots, u_{n}^{0}\right)$, where $u_{i}^{0}=\mu_{i}^{0}, i \in V$, and $u_{0}^{0}=L B_{0}-\sum_{i \in V} \mu_{i}$, represents a feasible solution of problem $D$ of $\operatorname{cost} L B_{0}$.

\subsubsection{Solving the Master Problem}

Instead of solving the master problem with the simplex, we use a dual-ascent heuristic based on the following theorem.

Theorem 1. Let $\boldsymbol{\lambda}=\left(\lambda_{0}, \lambda_{1}, \ldots, \lambda_{n}\right)$ be a vector of $n+1$ penalties, where penalties $\lambda_{i} \in \mathbb{R}, i \in V$, are associated with constraints (3.11) and penalty $\lambda_{0}$ with constraint (3.12). A feasible dual solution $\boldsymbol{u}$ of problem $P$ of $\operatorname{cost} z(P(\boldsymbol{\lambda}))=u_{0}+\sum_{i \in V} u_{i}$ is obtained as

$$
\left.\begin{array}{l}
u_{i}=\min _{\ell \in \widehat{\mathscr{H}}: a_{i \ell} \geq 1}\left\{\frac{c_{\ell}-\lambda_{0}-\sum_{i \in V} a_{i \ell} \lambda_{i}}{\sum_{i \in V} a_{i \ell}}\right\}+\lambda_{i}, i \in V \\
u_{0}=\lambda_{0}
\end{array}\right\}
$$

Proof. Consider a tour $\ell \in \widehat{\mathscr{H}}$. From expression (3.14), we derive

$$
u_{i} \leq \frac{c_{\ell}-\lambda_{0}-\sum_{i \in V} a_{i \ell} \lambda_{i}}{\sum_{i \in V} a_{i \ell}}+\lambda_{i}, \quad i \in V: a_{i \ell} \geq 1 .
$$

Thus, for each tour $\ell \in \widehat{\mathscr{H}}$, the following relation holds

$$
\sum_{i \in V} a_{i \ell} u_{i} \leq \sum_{i \in V} \frac{a_{i \ell}\left(c_{\ell}-\lambda_{0}-\sum_{i \in V} a_{i \ell} \lambda_{i}\right)}{\sum_{i \in V} a_{i \ell}}+\sum_{i \in V} a_{i \ell} \lambda_{i}=c_{\ell}-\lambda_{0}=c_{\ell}-u_{0}
$$


which corresponds to constraint (3.7) for tour $\ell \in \widehat{\mathscr{H}}$

$$
\sum_{i \in V} a_{i \ell} u_{i} \leq c_{\ell}-u_{0} \cdot \square
$$

To find a near-optimal solution $\boldsymbol{u}^{\prime}$ of the current master problem, we perform Maxit 1 subgradient iterations to modify the penalty vector $\boldsymbol{\lambda}$. Baldacci et al. [2008] showed that a valid subgradient of function $z(P(\boldsymbol{\lambda}))$ at point $\boldsymbol{\lambda}$ can be computed by associating with the current solution $\boldsymbol{u}^{\prime \prime}$ a nonnecessarily feasible solution $\boldsymbol{x}^{\prime \prime}$ of the current master problem such that $z(P(\boldsymbol{\lambda}))=\sum_{\ell \in \widehat{\mathscr{H}}} c_{\ell} x_{\ell}^{\prime \prime}$ defined as follows.

Let $\ell(i) \in \widehat{\mathscr{H}}$ be the index of the tour associated with $u_{i}, i \in V$, in expressions (3.14). Define variables $\xi_{\ell}^{i} \in\{0,1\}, \ell \in \widehat{\mathscr{H}}, i \in V$, as $\xi_{\ell(i)}^{i}=1$, for each vertex $i \in V$, and $\xi_{\ell}^{i}=0$, for each vertex $i \in V$ and each tour $\ell \in \widehat{\mathscr{H}} \backslash\{\ell(i)\}$. The solution $\boldsymbol{x}^{\prime \prime}$ is computed as $x_{\ell}^{\prime \prime}=\sum_{i \in V} \frac{a_{i \ell} \xi_{\ell}^{i}}{\sum_{i \in V} a_{i \ell}}$, for each tour $\ell \in \widehat{\mathscr{H}}$.

Let $\alpha_{i}, i \in V$, and $\alpha_{0}$ denote the values of the left-hand-side of constraints (3.11) and (3.12) with respect to solution $\boldsymbol{x}^{\prime \prime}$, respectively. The values of $\alpha_{i}, i \in V$, and $\alpha_{0}$ are used to update the penalty vector $\boldsymbol{\lambda}$ by means of the usual subgradient expressions, where $z_{U B}=1.2 z(P(\boldsymbol{\lambda}))$. We denote by $\boldsymbol{u}^{\prime}$ the final dual solution of the current master problem of cost $z(P(\boldsymbol{\lambda}))$ achieved by the procedure described above.

\subsubsection{Solving the Pricing Problem}

The pricing problem consists of finding a tour of minimum reduced cost with respect to the current dual solution $\boldsymbol{u}^{\prime}$ of the master. Define the modified arc cost $d_{i j}^{\prime}$ as

$$
d_{i j}^{\prime}= \begin{cases}d_{i j}-u_{j}^{\prime}, & (i, j) \in A \backslash A_{q} \\ d_{i q}-u_{0}^{\prime}, & (i, q) \in A_{q} .\end{cases}
$$

The reduced $\operatorname{cost} c_{\ell}^{\prime}=c_{\ell}-\sum_{i \in V} a_{i \ell} u_{i}^{\prime}-u_{0}^{\prime}$ of tour $\ell \in \widehat{\mathscr{H}}$ with respect to $\boldsymbol{u}^{\prime}$ is equal to the cost of the tour (i.e., $t$-tour or $n g$-tour or $n g L$-tour) using the modified arc costs $d_{i j}^{\prime}$ instead of $d_{i j}$. Therefore, to compute the tour of minimum reduced cost with respect to $\boldsymbol{u}^{\prime}$, it is sufficient to compute the associated DP recursion using $d_{i j}^{\prime}$ instead of $d_{i j}$.

If the cost of the minimum reduced cost tour is negative, the tour is added to the master problem and the bounding procedure performs another iteration; otherwise, $\boldsymbol{u}^{\prime}$ is a feasible dual solution of problem $P$ of cost $z(P(\boldsymbol{\lambda}))$, so the best-known lower bound $L B_{1}$ is update as $L B_{1}=z(P(\boldsymbol{\lambda}))$ and $\boldsymbol{u}^{\mathbf{1}}=\boldsymbol{u}^{\prime}$ if $L B_{1} \leq z(P(\boldsymbol{\lambda}))$. The bounding procedure terminates after a number of macro-iteration. 


\subsection{Computing Bounding Functions $b(S, t, i)$}

In this section, we describe how to compute bounding functions $b(S, t, i)$, introduced in $§ 3.3$, to reduce the state-space graph associated with the DP recursion (3.1).

We define a backward path $B=\left(\sigma_{B}=i_{k}, i_{k+1}, \ldots, i_{h}, q\right)$ as a path that starts from vertex $\sigma_{B}$ at time $t_{B} \in\left[e_{\sigma_{B}}, l_{\sigma_{B}}\right]$, visits each vertex in $V(B)=\left\{i_{k}, i_{k+1}, \ldots, i_{h}, q\right\}$ within its time window, and ends at vertex $q$ before time $l_{q}$.

Consider the forward path $F$ associated with $f(S, t, i)$. The least-cost salesman tour containing $F$ is obtained by adding, to $F$, a least-cost backward path $B$ of $\operatorname{cost} c(B)$ that starts from vertex $i$ at time $t^{\prime} \in\left[t, l_{i}\right]$ and visits all vertices $V^{\prime} \backslash S$.

The method to compute functions $b(S, t, i)$ is based on the following proposition. For a $D$ solution $\left(\boldsymbol{u}^{\mathbf{1}}, w^{1}\right)$ providing lower bound $L B_{1}$, define the modified arc costs $d_{i j}^{1}$ as

$$
d_{i j}^{1}= \begin{cases}d_{i j}-u_{j}^{1}, & (i, j) \in A \backslash A_{q}, \\ d_{i q}, & (i, q) \in A_{q} .\end{cases}
$$

Proposition 1. Let $l b(S, t, i)$ be a lower bound on the cost, using arc costs $d_{i j}^{1}$ instead of $d_{i j}$, of any backward path that starts from vertex $i \in V$ at time $t^{\prime} \in\left[t, l_{i}\right]$. A valid lower bound on the $\operatorname{cost} c(B)$ of a least-cost backward path $B$ that starts from vertex $i \in V$ at time $t^{\prime} \in\left[t, l_{i}\right]$ and visits vertices $V^{\prime} \backslash S$ is given by

$$
b(S, t, i)=l b(S, t, i)+\sum_{j \in V \backslash S} u_{i}^{1}
$$

Proof. Let $c^{1}(B)$ be the cost of backward path $B$ using arc costs $d_{i j}^{1}$. From the definition of $l b(S, t, i)$, we have

$$
l b(S, t, i) \leq c^{1}(B)
$$

Because $B$ visits each vertex $j \in V \backslash S$ exactly once, then

$$
c^{1}(B)=c(B)-\sum_{j \in V \backslash S} u_{i}^{1} .
$$

From expressions (3.15), (3.16) and (3.17) we obtain $b(S, t, i) \leq c(B)$.

In the following, we describe three methods to compute lower bound $l b(S, t, i)$ on $c(B)$ that are based on the three relaxations described in $\S 3.4$.

A backward $(t, i)$-path is a nonnecessarily elementary path $B$ that starts from vertex $i \in V^{\prime}$ at time $t \in\left[e_{i}, l_{i}\right]$, visits a subset of vertices (each once or more) within their time windows, and ends at vertex $q$ before time $l_{q}$. We denote by $f^{-1}(t, i)$ the cost of a least-cost backward $(t, i)$-path without 2 -vertex loops. 
A backward ng-path $(N G, t, i)$ is a nonnecessarily elementary path $B=\left(i=i_{k}, i_{k+1}, \ldots\right.$, $i_{h}, i_{q}$ ) that starts from vertex $i \in V^{\prime}$ at time $t \in\left[e_{i}, l_{i}\right]$, visits a subset of vertices (each once or more) within their time windows such that $N G=\Pi^{-1}(B)$, ends at vertex $q$ before $l_{q}$, and such that $i \notin \Pi^{-1}\left(B^{\prime}\right)$, where path $B^{\prime}$ is $B^{\prime}=\left(i_{k+1}, \ldots, i_{h}, i_{q}\right)$ and must be a backward $n g$-path. For each backward path $B$, the set $\Pi^{-1}(B)$ is defined as

$$
\Pi^{-1}(B)=\left\{i_{r}: i_{r} \in \bigcap_{s=k}^{r-1} N_{i_{s}}, r=k+1, \ldots, h\right\} \bigcup\left\{i_{k}\right\} .
$$

We denote by $f^{-1}(N G, t, i)$ the cost of a least-cost backward $n g$-path $(N G, t, i)$.

An example of backward $n g$-path (NG,t,i) is the following. Let $B=(9,6,7,8,9,0)$ be a backward path that starts from vertex 9 , and let $N_{6}=\{6,7,8\}, N_{7}=\{6,7,8\}, N_{8}=$ $\{7,8,9\}$ and $N_{9}=\{6,7,9\}$. Thus, path $B^{\prime}$ is $B^{\prime}=(6,7,8,9,0)$ and $\Pi\left(B^{\prime}\right)=\{6,7,8\}$ because 6 is the first visited vertex and $7 \in N_{6}, 8 \in N_{6} \cap N_{7}$, and $9 \notin N_{6} \cap N_{7} \cap N_{8}$. Because $B^{\prime}$ is an $n g$-path and $9 \notin \Pi\left(B^{\prime}\right), B$ is an $n g$-path.

For a given longest path $L=\left(i_{0}=p, i_{1}, \ldots, i_{k}, i_{k+1}, \ldots, i_{h}, i_{h+1}=q\right)$, let $\bar{V}_{k}$ be the subset of vertices that must or can be visited in between vertices $i_{k}$ and $i_{k+1}$, $k=0, \ldots, h$, that is

$$
\bar{V}_{k}=\left\{j \in V \backslash\left\{i_{k+1}\right\}: e_{i_{k}}+\vec{t}_{i_{k} j} \leq l_{j} \text { and } \max \left\{e_{i_{k}}+\vec{t}_{i_{k} j}, e_{j}\right\}+\vec{t}_{j i_{k+1}} \leq l_{i_{k+1}}\right\}
$$

A backward $n g L(k)$-path $(N G, t, i)$ is a backward $n g$-path that starts from vertex $i \in \bar{V}_{k}$ and visits the vertices $\left\{i_{k+1}, \ldots, i_{h}, i_{q}\right\}$ exactly once. We denote by $f_{k}^{-1}(N G, t, i)$ the cost of a least-cost backward $n g L(k)$-path.

Functions $f^{-1}(t, i), f^{-1}(N G, t, i)$ and $f_{k}^{-1}(N G, t, i)$ can be computed with the same DP recursions used to compute $f(t, i), f(N G, t, i)$ and $f(N G, k, t, i)$ on the TSPTW instance resulting from the following operations: (i) for each vertex $i \in V^{\prime}$, replace time window $\left[e_{i}, l_{i}\right]$ with time window $\left[l_{q}-l_{i}, l_{q}-e_{i}\right]$; (ii) replace the cost and time matrices $\left[d_{i j}\right]$ and $\left[t_{i j}\right]$ with their transposed matrices $\left[d_{i j}\right]^{T}$ and $\left[t_{i j}\right]^{T}$.

Let functions $f^{-1}(t, i), f^{-1}(N G, t, i)$ and $f_{k}^{-1}(N G, t, i)$ be computed replacing the arc $\operatorname{costs} d_{i j}$ with $d_{i j}^{1}$. These functions allow for the computing of the lower bound $l b(S, t, i)$ as follows

a) From functions $f^{-1}(t, i)$, we derive

$$
l b(S, t, i)=\min _{t^{\prime} \in\left[t, l_{i}\right]}\left\{f^{-1}\left(t^{\prime}, i\right)\right\} .
$$

b) From functions $f^{-1}(N G, t, i)$, we derive

$$
l b(S, t, i)=\min _{N G \subseteq N_{i}: \begin{array}{c}
N G \ni i, N G \cap S=\{i\} \\
t^{\prime} \in\left[t, l_{i}\right]
\end{array}}\left\{f^{-1}\left(N G, t^{\prime}, i\right)\right\} .
$$


c) From functions $f_{k}^{-1}(N G, t, i)$, we derive

$$
l b(S, t, i)=\min _{N G \subseteq N_{i}: N G \ni i, N G \cap S=\{i\},}\left\{f_{k^{\prime}}^{-1}\left(N G, t^{\prime}, i\right)\right\},
$$

where $k^{\prime}$ is defined as follows

i) $i \notin V(L): k^{\prime}$ is the index of the first vertex $i_{k^{\prime}}$ of the longest path $L$ such that $i_{k^{\prime}} \notin S$;

ii) $i \in V(L): k^{\prime}$ is the index of the vertex of $L$ such that $i_{k^{\prime}}=i$.

\subsection{Computational Results}

This section reports on the computational experiments of our algorithm, which was implemented in C and compiled with Visual Studio 2008. The runs were performed on a Sony Vaio P8400 (Intel Core 2 Duo, $2.26 \mathrm{GHz}$ ), equipped with 4 GB of RAM.

We denote by BMR.ng the exact algorithm of $\S 3.3$ using either the $n g$-tour or the $n g L$ tour relaxations, according to the criterion described in $\$ 3.7 .1$, in computing the lower bound and the bounding functions $b(S, t, i)$. We denote by BMR.t the exact method when the $t$-tour relaxation is used in computing the lower bound and the bounding functions.

We tested both BMR.ng and BMR.t on the seven classes of instances available at http: //iridia.ulb.ac.be/ manuel/tsptw-instances and the class proposed by Mingozzi et al. [1997]. The classes proposed by Langevin et al. [1993], Dumas et al. [1995], and Mingozzi et al. [1997] were easily solved (the most difficult instance was solved in 12 seconds by BMR.ng), so relative results are omitted. The results on the other five classes are reported in the following and compared with the following state-of-the-art exact methods:

- the branch-and-cut of Ascheuer et al. [2001] (hereafter AFG) - runs performed on a Sun Sparc Station 10 with a time limit (tl) of 5 hours (i.e., tl $=18,000$ seconds);

- the constraint programming based method of Focacci et al. [2002] (hereafter FLM) - Pentium III $700 \mathrm{MHz}$ with $128 \mathrm{MB}$ of RAM (tl = 1,800 seconds). The results reported in the tables are, on each instance, the best achieved by the two versions of their method (i.e., AP-bound and Lagrangean-bound).

- the branch-and-cut of Dash et al. [2012] (hereafter DGLT) - workstation Intel $2.40 \mathrm{GHz}$ running under Suse Linux 10.1 ( $\mathrm{tl}=18,000$ seconds); 
TABLE 3.1: Results of BMR.ng on a representative set of instances

\begin{tabular}{lccccccccccc}
\hline Inst & $\left|V^{\prime}\right|$ & $|L|$ & Prec & $z^{*}$ & Rel & $\Delta\left(N_{i}\right)$ & $L B$ & $\% L B$ & $T_{L B}$ & $|\mathscr{F}|$ & $T_{\text {tot }}$ \\
\hline n150w120.002 & 152 & 13 & 77.8 & 677 & $n g$ & 11 & 663.3 & 97.98 & 69.7 & - & - \\
& & & & & $n g$ & 13 & 663.8 & 98.05 & 124.8 & - & - \\
& & & & & $n g L$ & 11 & 668.0 & 98.66 & 77.2 & 14,473 & 211.3 \\
& & & & & $n g L$ & 13 & 668.4 & 98.73 & 130.5 & 12,149 & 260.6 \\
\hline n200w120.005 & \multirow{2}{*}{202} & \multirow{2}{*}{14} & \multirow{2}{*}{79.6} & 840 & $n g$ & 11 & 827.5 & 98.51 & 155.0 & - & - \\
& & & & & $n g$ & 13 & 828.9 & 98.68 & 195.1 & - & - \\
& & & & & $n g L$ & 11 & 831.5 & 98.99 & 204.9 & - & - \\
& & & & & $n g L$ & 13 & 833.2 & 99.19 & 237.4 & 29,072 & 574.3 \\
\hline rbg152.3 & \multirow{2}{*}{12} & \multirow{2}{*}{81.3} & \multirow{2}{*}{1,539} & $n g$ & 11 & $1,536.2$ & 99.82 & 47.3 & 22,015 & 192.5 \\
& & & & & $n g$ & 13 & $1,536.7$ & 99.85 & 64.7 & 17,387 & 206.7 \\
& & & & & $n g L$ & 11 & $1,537.7$ & 99.92 & 73.3 & 17,191 & 201.9 \\
& & & & & $n g L$ & 13 & $1,538.2$ & 99.95 & 81.5 & 14,151 & 213.2 \\
\hline rbg233.2 & 22 & \multirow{2}{*}{90.9} & 2,188 & $n g$ & 11 & $2,187.0$ & 99.95 & 72.1 & 9,200 & 124.8 \\
& & & & & $n g$ & 13 & $2,187.0$ & 99.95 & 92.2 & 8,906 & 136.9 \\
& & & & & $n g L$ & 11 & $2,187.0$ & 99.95 & 231.1 & 6,750 & 364.3 \\
& & & & & $n g L$ & 13 & $2,187.0$ & 99.95 & 253.3 & 5,265 & 359.6 \\
\hline
\end{tabular}

- the DP algorithm of Li [2009] (hereafter LI) - Lenovo Thinkstation with 4 Intel processors at $2 \mathrm{GHz}, 4 \mathrm{BG}$ of RAM and a Linux operating system (no time limit imposed).

These exact methods were not tested on all five instance classes. In the tables of this section, "tl" indicates that the time limit was reached.

According to SPEC (http://www.spec.org/benchmarks.html), our machine is $10 \%$ slower than that of Dash et al. [2012] and 10\% faster than that of Li [2009]. No benchmarks are available to compare our machine with those used by Ascheuer et al. [2001] and Focacci et al. [2002]. Nevertheless, our machine is clearly faster (say, 6-10 times faster) than these two machines.

\subsubsection{Parameter Setting}

To find a parameter setting for BMR.ng on all instances, we performed preliminary tests applying both $n g$-tour and $n g L$-tour relaxations and varying parameter $\Delta\left(N_{i}\right)$.

In Table 3.1, we present such results on a selected set of representative instances. The columns report the instance name (Inst), the number of vertices of graph $G\left(\left|V^{\prime}\right|\right)$, the length of the longest path $L$ of the precedence graph $\widehat{G}(|L|)$, the percentage of precedences $($ Prec $)$ computed as Prec $=100 \frac{2|\widehat{A}|}{(n+2)(n+1)}$, and the optimal solution cost $\left(z^{*}\right)$. Then, we indicate the tour relaxation used $(R e l)$, which can be either $n g$-tour or $n g L$-tour, and the setting of parameter $\Delta\left(N_{i}\right)$. Finally, the lower bound achieved $(L B)$ is reported with the percentage $\left(\% L B=100 L B / z^{*}\right)$, the time (in seconds) to compute the lower bound $\left(T_{L B}\right)$, the cardinality (in thousands) of the set $\mathscr{F}$ generated by the exact algorithm of $\S 3.3(|\mathscr{F}|)$, and the total computing time in seconds $\left(T_{\text {tot }}\right)$. 


\begin{tabular}{|c|c|c|c|c|c|c|c|c|c|c|c|c|c|c|c|c|c|}
\hline \multirow[b]{2}{*}{ Inst } & \multirow[b]{2}{*}{$\left|V^{\prime}\right|$} & \multirow[b]{2}{*}{$z^{*}$} & \multicolumn{6}{|c|}{ BMR.ng } & \multicolumn{2}{|c|}{ BMR.t } & \multicolumn{2}{|c|}{$\mathrm{AFG}$} & \multicolumn{2}{|c|}{ FLM } & \multicolumn{2}{|c|}{ DGLT } & \multirow{2}{*}{$\frac{\mathrm{LI}}{T_{t o t}}$} \\
\hline & & & Prec & $L B$ & $\% L B$ & $T_{L B}$ & $|\mathscr{F}|$ & $T_{t o t}$ & $\% L B$ & $T_{t o t}$ & $\% L B$ & $T_{t o t}$ & $\% L B$ & $T_{t o t}$ & $\% L B$ & $T_{t o t}$ & \\
\hline cbg010a & 12 & 149 & 72.7 & 149.0 & 100.0 & 0.1 & & 0.1 & 99.5 & 0.8 & 99.3 & 0.1 & 99.3 & 0.0 & 100.0 & 0.0 & 0.1 \\
\hline g017 & 17 & 148 & 72.1 & 148.0 & 100.0 & 0.1 & & .1 & 97.2 & 0.7 & 100.0 & 0.8 & 93.2 & 0.1 & 99.3 & 0.0 & 0.1 \\
\hline g017.2 & 17 & 107 & 41.2 & 107.0 & 100.0 & 0.1 & & 0.1 & 100.0 & 0.6 & 100.0 & 0.0 & 93.5 & 0.0 & 100.0 & 0.0 & 19.9 \\
\hline g016a & 18 & 179 & 83.0 & 179.0 & 100.0 & 0.1 & & 0.1 & 100.0 & 2.2 & 98.9 & 0.2 & 93.3 & 0.1 & 100.0 & 0.0 & 0.1 \\
\hline g016b & 18 & 142 & 56.9 & 142.0 & 100.0 & 0.2 & & 0.2 & 95.9 & 4.8 & 93.7 & 8.8 & 88.7 & 0.1 & 97.2 & 0.2 & 0.1 \\
\hline g017a & 19 & 146 & 49.1 & 146.0 & 100.0 & 0.3 & & 0.3 & 100.0 & 0.4 & 100.0 & 0.1 & 100.0 & 0.1 & 100.0 & 0.0 & 10.3 \\
\hline & 21 & 217 & 91.9 & 217.0 & 100.0 & 0.2 & & 0.2 & 100.0 & 0.4 & 100.0 & 0.0 & 100.0 & .0 & 100.0 & & 0.1 \\
\hline $019 \mathrm{~b}$ & 21 & 182 & 61.4 & 182.0 & 100.0 & 0.3 & & 0.3 & 99.4 & 2.8 & 98.9 & 54.5 & 96.2 & 0.2 & 99.5 & 0.3 & 0.2 \\
\hline $19 c$ & 21 & 190 & 45.7 & 190.0 & 100.0 & 0.5 & & 0.5 & 98.5 & 1.4 & 5.8 & 8.7 & 94.2 & .3 & 96.8 & 0.9 & 35.3 \\
\hline & 21 & 344 & 78.1 & 344.0 & 100.0 & 0.3 & & 0.3 & 98.8 & 2.6 & 99.7 & 0.7 & 98.3 & .0 & 100.0 & 0.2 & 0.1 \\
\hline & 21 & 190 & 45. & 190.0 & 100.0 & 0.5 & & 0.5 & 98.6 & 2.5 & & & 94.2 & .3 & 96.8 & 0.9 & 35.5 \\
\hline & 21 & 182 & 43.3 & 182.0 & 100.0 & 0.4 & & 0.4 & 100.0 & 0.8 & 100.0 & 0.2 & 98.4 & 0.2 & 100.0 & & 48.0 \\
\hline g021.3 & 21 & 182 & 42.9 & 182.0 & 100.0 & 1.4 & & 1.4 & 98.9 & 3.0 & 97.8 & 27.1 & 91.2 & 0.4 & 98.4 & 2.7 & 101.2 \\
\hline 021.4 & 21 & 179 & 40.5 & 179.0 & 100.0 & 1.2 & 1 & 1.4 & 99.7 & 2.2 & 8.9 & 5.8 & 92.7 & .3 & 100.0 & 0.2 & 692.8 \\
\hline 021.5 & 21 & 169 & 39. & 169.0 & 100.0 & 1.1 & & 1.1 & 99.6 & 1.9 & 98.8 & 6.6 & 94.7 & 0.2 & 100.0 & 0.3 & $1,320.5$ \\
\hline 021.6 & 21 & 134 & 24 & 134.0 & 100.0 & 0.6 & 1 & 0.7 & 100.0 & 1.4 & & & 95.5 & .7 & 100.0 & 0.3 & $7,876.4$ \\
\hline & 21 & 133 & 19. & 133.0 & 100.0 & 1.5 & 1 & & 98.5 & 2.1 & 96. & 4.3 & 94.0 & 6 & 100.0 & 0.6 & \\
\hline 8 & 21 & 132 & 18 & 132.0 & 100.0 & 1.6 & 15 & 1.8 & 98.4 & 2.3 & 97.7 & 17.4 & 97.0 & .6 & 98.5 & 2.8 & \\
\hline & 21 & & & 132.0 & 100.0 & 3.1 & 18 & 3.3 & 98.0 & 2.2 & 97.0 & 26.1 & 94.7 & & 98.5 & 2.8 & \\
\hline & 22 & 210 & 53. & 210.0 & 100.0 & 0.7 & & 0.7 & 100.0 & 0.9 & 100.0 & 0.2 & 98.6 & 0.0 & 100.0 & 0.0 & 10.5 \\
\hline $027 \mathrm{a}$ & 29 & 268 & 43.1 & 267.9 & 100.0 & 1.3 & 1 & 1.5 & 99.5 & 7.3 & 99.3 & 2.2 & 97.0 & 0.2 & 99.3 & 1.4 & 0.7 \\
\hline & 33 & & 75 . & 328.0 & 100.0 & 0.5 & & 0.5 & & 7.4 & & & 97 & 7 & 100.0 & & 0.1 \\
\hline & 35 & 433 & 77.6 & 433.0 & 100.0 & 0.5 & & 0.5 & 99.3 & 6.5 & 100.0 & 1. & 92.8 & 1.0 & 99.8 & .9 & 0.2 \\
\hline & 36 & 403 & & 402.9 & 100.0 & 3.2 & 5 & 3.5 & 99.2 & 9.4 & & & 97.0 & 55.2 & 100.0 & 1.9 & 1.0 \\
\hline & 37 & 254 & 76 . & 254.0 & 100.0 & 0.6 & & 0.6 & 98.8 & 12.7 & 100.0 & 1.8 & 87.0 & 3.5 & 100.0 & 0.2 & 0.1 \\
\hline rbg035a.2 & 37 & 166 & 70.9 & 166.0 & 100.0 & 23.5 & 6 & 24.1 & 98.4 & 20.1 & 95.2 & 64.8 & 93.4 & 36.8 & 100.0 & 5.3 & \\
\hline & 40 & & 78.1 & 466.0 & 100.0 & 2.0 & & 2.0 & 100.0 & 6.2 & 100.0 & $4,232.2$ & 100.0 & 0.2 & 100.0 & 1.5 & 0.5 \\
\hline & 42 & 386 & 79 & 384.9 & & & 1 & & 97 & 5.7 & 92 & 751.8 & 73.1 & 738.1 & 96.6 & 3.6 & 0.8 \\
\hline g050a & 52 & 414 & 44.2 & 414.0 & 100.0 & 16.1 & 4 & 16.6 & 99.7 & 24.4 & 100.0 & 18.6 & 98.8 & 95.6 & 100.0 & 24.5 & \\
\hline & 57 & 814 & & 814.0 & & 1.8 & 1 & & & 7.3 & & 6.4 & & 2.5 & 100.0 & 3.5 & 2.4 \\
\hline & 69 & 1,048 & 88.8 & $1,048.0$ & & 1.9 & 1 & 2.2 & 99.4 & 17.6 & 99.9 & 5.9 & & 4.0 & 100.0 & 3.6 & 3.2 \\
\hline rbg125a & 127 & 1,409 & 92.8 & $1,409.0$ & 100.0 & 5.6 & 2 & 6.4 & 99.3 & 71.0 & 99.5 & 229.8 & 98.2 & $\mathrm{tl}$ & 100.0 & 9.6 & 3.5 \\
\hline & & & & & 100.0 & 2.3 & & 2.4 & 99.1 & 7.2 & 98.5 & 171.5 & 95.0 & 30.5 & 99.4 & 2.1 & 376.4 \\
\hline & & & & & & & & 32 & & 32 & & 32 & & 31 & & 32 & \\
\hline
\end{tabular}


Table 3.1 shows that increasing parameter $\Delta\left(N_{i}\right)$ lets us compute better lower bounds and generate smaller state-space graphs when solving the problem to optimality. Nonetheless, because the complexity of computing the recursions 3.3 and 3.5 and testing Fathoming 1 increases, the computing time to compute the lower bound and the total computing time may increase.

The $n g L$-tour relaxation dominates the $n g$-tour relaxation but is more time consuming. At the same time, the lower bound that can be achieved with the $n g L$-tour relaxation is sometimes just slightly better or the same bound that can be achieved with the $n g$-tour relaxation. In particular, we can see that the higher is the number of arcs in the precedence digraph $\widehat{G}$ (i.e., Prec), the lower is the increment of the lower bound when applying $n g L$-tour relaxation instead of the $n g$-tour relaxation.

Notice that the $n g L$-tour relaxation allows us to solve a few instances that cannot be solved with the $n g$-tour relaxation. Thus, BMR.ng uses the $n g$-tour relaxation if Prec $\geq 80$ and the $n g L$-tour relaxation otherwise.

In computing the lower bound $L B$ (see $\S 3.5 .3$ ), parameter $\Delta\left(N_{i}\right)$ was set equal to 11 when using the $n g$-tour relaxation and equal to 13 when using $n g L$-tour relaxation. In computing bounding functions $b(S, t, i)$ (see $\S 3.6$ ), parameter $\Delta\left(N_{i}\right)$ was set equal to 13 for both relaxations.

Finally, in the column generation method described in $§ 3.5$, for both BMR.ng and BMR.t we set Maxit $0=200$ and Maxit $1=150$. In the exact method, due to memory limits, the cardinality of the state set $\mathscr{F}$ is limited to $10^{8}$. Both BMR.ng and BMR.t terminate prematurely when this memory limit is reached.

\subsubsection{Ascheuer Instances}

The Ascheuer class consists of 50 asymmetric real-world instances with up to 233 vertices. Both travel costs and times are integer values. Travel times satisfy the triangle inequality.

Like Dash et al. [2012], we present the results on this class dividing the 50 instances into 32 easy instances (those solved by Ascheuer et al. [2001] within the time limit of 5 hours) and the remaining 18 hard instances.

Table 3.2 presents the computational results on easy instances. The columns mean the same as in Table 3.1. For AFG, FLM and DGLT, column $(\% L B)$ reports the percentage deviation of the lower bound at the root node of the decision tree. The last two lines of the table indicate averages on the percentage deviation of the lower bounds, the computing times of the instances solved to optimality and the number of instances solved by each method. 
TABLE 3.3: Results on hard Ascheuer instances

\begin{tabular}{|c|c|c|c|c|c|c|c|c|c|c|c|c|c|c|c|c|}
\hline \multirow[b]{2}{*}{ Inst } & \multirow[b]{2}{*}{$\left|V^{\prime}\right|$} & \multirow[b]{2}{*}{$z^{*}$} & \multicolumn{6}{|c|}{ BMR.ng } & \multicolumn{2}{|c|}{ BMR.t } & \multirow{2}{*}{$\frac{\mathrm{AFG}}{\% L B}$} & \multicolumn{2}{|c|}{ FLM } & \multicolumn{2}{|c|}{ DGLT } & \multirow{2}{*}{$\frac{\mathrm{LI}}{T_{t o t}}$} \\
\hline & & & Prec & $L B$ & $\% L B$ & $T_{L B}$ & $|\mathscr{F}|$ & $T_{t o t}$ & $\% L B$ & $T_{t o t}$ & & $\% L B$ & $T_{t o t}$ & $\% L B$ & $T_{t o t}$ & \\
\hline rbg041a & 43 & 402 & 77.3 & 402.0 & 100.0 & 2.0 & & 2.0 & 95.4 & 4.2 & 89.8 & 87.6 & $\mathrm{tl}$ & 97.5 & 146.8 & 2.2 \\
\hline rbg042a & 44 & 411 & 71.6 & 411.0 & 100.0 & 6.6 & & 6.6 & 97.7 & 5.0 & 95.9 & 90.0 & 149.8 & 98.3 & 188.3 & 35.6 \\
\hline rbg048a & 50 & 487 & 47.8 & 487.0 & 100.0 & 12.3 & & 12.3 & 100.0 & 14.7 & 93.2 & 91.2 & tl & 100.0 & 129.2 & - \\
\hline rbg049a & 51 & 484 & 63.4 & 472.1 & 97.5 & 19.5 & 6 & 20.4 & 95.7 & 13.7 & 84.3 & 83.3 & tl & 95.9 & $\mathrm{tl}$ & - \\
\hline rbg050b & 52 & 512 & 61.1 & 504.8 & 98.6 & 19.1 & 2 & 19.9 & 96.5 & 8.1 & 87.3 & 87.1 & $\mathrm{tl}$ & 96.3 & $\mathrm{tl}$ & - \\
\hline rbg050c & 52 & 526 & 49.8 & 522.4 & 99.3 & 20.3 & 93 & 21.7 & 98.5 & 23.1 & 96.4 & 95.8 & $\mathrm{tl}$ & 98.3 & $\mathrm{tl}$ & - \\
\hline rbg086a & 88 & 1,051 & 91.7 & $1,050.0$ & 99.9 & 2.1 & 1 & 2.5 & 99.1 & 14.5 & 99.1 & 97.3 & tl & 99.8 & 4.9 & 1.9 \\
\hline rbg092a & 94 & 1,093 & 89.8 & $1,091.6$ & 99.9 & 6.7 & 3 & 7.3 & 99.7 & 14.2 & 99.2 & 98.8 & $\mathrm{tl}$ & 99.8 & 90.4 & 9.0 \\
\hline $\operatorname{rbg} 132.2$ & 132 & 1,083 & 88.2 & $1,080.2$ & 99.7 & 45.5 & 294 & 47.9 & 99.4 & 80.5 & 97.2 & n.a. & & 99.8 & $2,761.1$ & - \\
\hline rbg132 & 132 & 1,360 & 94.2 & $1,360.0$ & 100.0 & 10.6 & 1 & 11.3 & 98.9 & 48.9 & 97.3 & n.a. & & 99.5 & 37.6 & 6.1 \\
\hline $\operatorname{rbg} 152.3$ & 152 & 1,539 & 81.3 & $1,536.2$ & 99.8 & 47.3 & 20,971 & 166.9 & 99.4 & 238.8 & 98.8 & n.a. & & 99.9 & $10,353.3$ & - \\
\hline rbg152 & 152 & 1,783 & 94.0 & $1,783.0$ & 100.0 & 18.8 & 3 & 19.8 & 99.3 & 36.8 & 98.7 & n.a. & & 99.8 & 43.7 & 11.5 \\
\hline rbg172a & 174 & 1,799 & 93.9 & $1,799.0$ & 100.0 & 37.1 & 4 & 38.4 & 99.2 & 455.7 & 98.8 & n.a. & & 99.8 & 425.5 & 544.3 \\
\hline rbg193.2 & 193 & 2,017 & 89.1 & $2,015.3$ & 99.9 & 50.8 & 414 & 54.4 & 99.6 & 76.3 & 97.6 & n.a. & & 99.7 & tl & - \\
\hline rbg193 & 193 & 2,414 & 94.5 & $2,414.0$ & 100.0 & 46.4 & 16 & 47.9 & 99.8 & 65.9 & 98.8 & n.a. & & 100.0 & 159.6 & $3,165.7$ \\
\hline rbg201a & 203 & 2,189 & 94.8 & $2,189.0$ & 100.0 & 54.3 & 4 & 56.0 & 99.3 & 716.2 & 98.6 & n.a. & & 99.9 & 462.7 & 581.1 \\
\hline rbg233.2 & 233 & 2,188 & 90.9 & $2,187.0$ & 100.0 & 72.1 & 8,969 & 120.6 & 99.8 & 124.4 & 98.1 & n.a. & & 99.7 & tl & - \\
\hline rbg233 & 233 & 2,689 & 95.4 & $2,688.0$ & 100.0 & 78.9 & 35 & 81.0 & 99.5 & 319.3 & 98.0 & n.a. & & 100.0 & 749.4 & $1,433.3$ \\
\hline Avg & & & & & 99.7 & 30.6 & & 40.9 & 98.7 & 125.6 & 95.9 & 91.4 & 149.8 & 99.1 & $1,196.3$ & 579.0 \\
\hline Solved & & & & & & & & 18 & & 18 & & & 1 & & 13 & 10 \\
\hline
\end{tabular}


Table 3.2 shows that the lower bound computed by BMR.ng closes the gap on all but 3 instances. BMR.ng solves all 32 instances with the same performance of DGLT.

Table 3.3 reports the results on hard Ascheuer instances. In column $z^{*}$ the optimal value is in bold when instances were solved for the first time by BMR.ng. Table 3.3 shows that BMR.ng solves the 5 open instances and outperforms the other exact methods.

\subsubsection{Pesant Instances}

The Pesant class consists of 27 symmetric instances proposed by Pesant et al. [1998] with 21 to 46 vertices. Travel costs and times are the Euclidean distances truncated to four decimal places. Travel times include service times and satisfy the triangle inequality.

Table 3.4 reports the results on Pesant instances. The lower bounds computed by BMR.ng are of excellent quality. BMR.ng and BMR.t solve all instances of this class and clearly outperform FLM, DGLT and LI.

\subsubsection{Potvin Instances}

The Potvin class consists of 28 symmetric instances introduced by Potvin and Bengio [1996] with 15 to 47 vertices (we neglect instances with fewer than 10 vertices). The instances feature x-y coordinates. Travel times are Euclidean and include service time at the starting vertex of each arc. Travel costs and travel times coincide.

In Table 3.5, we compare BMR.ng and BMR.t with LI. In column $z^{*}$, the optimal solution cost is rounded to two decimal places. The last column indicates the computing time of LI (a "_" is reported when the instance was not solved).

From Table 3.5, both BMR.ng and BMR.t compare favorably with LI. BMR.ng is clearly superior to both BMR.t and LI because it solves all instances.

\subsubsection{Gendreau Instances}

The Gendreau class is made up of 28 groups of 5 instances proposed by Gendreau et al. [1998]. Each group consists of five instances having the same number of vertices and the same time windows width. The instances are obtained from the instances of Dumas et al. [1995] extending the time windows and resulting in time windows ranging from 80 to 200 time units in increments of 20 . The number of vertices ranges from 22 to 102 . 
TABLE 3.4: Results on Pesant instances

\begin{tabular}{|c|c|c|c|c|c|c|c|c|c|c|c|c|c|c|c|}
\hline \multirow[b]{2}{*}{ Inst } & \multirow[b]{2}{*}{$\left|V^{\prime}\right|$} & \multirow[b]{2}{*}{$z^{*}$} & \multicolumn{6}{|c|}{ BMR.ng } & \multicolumn{2}{|c|}{ BMR.t } & \multicolumn{2}{|c|}{ FLM } & \multicolumn{2}{|c|}{ DGLT } & \multirow{2}{*}{$\frac{\mathrm{LI}}{T_{\text {tot }}}$} \\
\hline & & & Prec & $L B$ & $\% L B$ & $T_{L B}$ & $|\mathscr{F}|$ & $T_{t o t}$ & $\% L B$ & $T_{t o t}$ & $\% L B$ & $T_{t o t}$ & $\% L B$ & $T_{t o t}$ & \\
\hline rc201.0 & 27 & 378.62 & 84.3 & 378.62 & 100.0 & 0.1 & & 0.1 & 100.0 & 0.1 & 100.0 & 0.2 & 100.0 & 0.0 & 0.1 \\
\hline rc201.1 & 30 & 374.70 & 85.3 & 374.70 & 100.0 & 0.2 & & 0.2 & 99.0 & 0.3 & 68.4 & 1.8 & 100.0 & 0.0 & 0.1 \\
\hline rc201.2 & 30 & 427.65 & 83.7 & 427.65 & 100.0 & 0.2 & & 0.2 & 100.0 & 0.1 & 99.0 & 0.4 & 100.0 & 0.0 & 0.1 \\
\hline rc201.3 & 21 & 232.54 & 82.9 & 232.54 & 100.0 & 0.1 & & 0.1 & 99.9 & 0.1 & 93.7 & 0.1 & 98.7 & 0.1 & 0.1 \\
\hline rc202.0 & 27 & 246.22 & 45.3 & 246.22 & 100.0 & 0.4 & & 0.4 & 100.0 & 0.2 & 98.2 & 1.0 & 100.0 & 0.2 & 63.0 \\
\hline rc202.1 & 24 & 206.53 & 55.1 & 206.53 & 100.0 & 0.4 & & 0.4 & 94.8 & 0.4 & 71.1 & 3.8 & 100.0 & 0.1 & 1.1 \\
\hline $\mathrm{rc} 202.2$ & 29 & 341.77 & 61.3 & 341.77 & 100.0 & 0.2 & & 0.2 & 94.6 & 0.8 & 82.5 & 3.1 & 100.0 & 0.1 & 0.4 \\
\hline $\mathrm{rc} 202.3$ & 28 & 367.85 & 50.5 & 367.85 & 100.0 & 0.3 & & 0.3 & 97.9 & 0.6 & 76.8 & 33.1 & 100.0 & 0.2 & 5.1 \\
\hline rc203.0 & 37 & 377.45 & 24.0 & 376.22 & 99.7 & 4.2 & 2 & 4.6 & 83.4 & 31.0 & 79.0 & tl & 95.4 & $3,437.7$ & - \\
\hline rc203.1 & 39 & 356.99 & 25.1 & 356.66 & 99.9 & 14.1 & 1 & 14.5 & 89.1 & 20.4 & 84.4 & tl & 97.6 & $2,722.7$ & - \\
\hline rc203.2 & 30 & 337.47 & 42.3 & 337.47 & 100.0 & 0.5 & & 0.5 & 94.1 & 2.3 & 87.5 & 94.3 & 100.0 & 0.9 & 392.7 \\
\hline rc204.0 & 34 & 221.45 & 16.8 & 221.45 & 100.0 & 1.7 & & 1.7 & 93.1 & 2.0 & 96.8 & 352.8 & 100.0 & 2.8 & - \\
\hline rc204.1 & 30 & 205.37 & 14.7 & 205.37 & 100.0 & 4.3 & & 4.3 & 92.3 & 9.0 & 98.9 & 3.4 & 99.0 & 14.9 & \\
\hline $\mathrm{rc} 204.2$ & 42 & 378.40 & 14.6 & 374.77 & 99.0 & 33.5 & 7 & 36.4 & 92.3 & 29.4 & 87.3 & $\mathrm{tl}$ & 96.6 & $\mathrm{tl}$ & - \\
\hline $\mathrm{rc} 205.0$ & 28 & 251.65 & 60.1 & 251.65 & 100.0 & 0.3 & & 0.3 & 100.0 & 1.4 & 91.7 & 7.9 & 100.0 & 0.1 & 1.9 \\
\hline rc205.1 & 24 & 271.22 & 67.8 & 271.22 & 100.0 & 0.2 & & 0.2 & 98.9 & 0.2 & 88.7 & 0.2 & 100.0 & 0.0 & 0.4 \\
\hline $\mathrm{rc} 205.2$ & 30 & 434.70 & 68.5 & 434.70 & 100.0 & 0.4 & & 0.4 & 92.7 & 1.2 & 67.8 & $1,289.1$ & 100.0 & 0.3 & 0.3 \\
\hline rc205.3 & 26 & 361.24 & 64.6 & 361.24 & 100.0 & 0.2 & & 0.2 & 100.0 & 0.2 & 72.4 & 4.7 & 100.0 & 0.0 & 0.1 \\
\hline rc206.0 & 37 & 485.23 & 62.6 & 485.23 & 100.0 & 0.8 & & 0.8 & 90.3 & 1.7 & 89.1 & 338.1 & 97.5 & 73.3 & 1.6 \\
\hline rc206.1 & 35 & 334.73 & 57.8 & 334.73 & 100.0 & 1.0 & & 1.0 & 96.3 & 0.8 & 96.0 & 22.9 & 98.2 & 85.0 & 42.5 \\
\hline $\mathrm{rc} 206.2$ & 34 & 335.37 & 55.8 & 335.37 & 100.0 & 0.9 & & 0.9 & 98.7 & 0.9 & 96.0 & 24.1 & 98.8 & 84.8 & 189.5 \\
\hline rc207.0 & 38 & 436.69 & 38.7 & 436.69 & 100.0 & 0.6 & & 0.6 & 95.1 & 2.0 & 75.9 & 572.0 & 99.5 & 19.0 & $18,691.8$ \\
\hline rc207.1 & 35 & 396.36 & 37.8 & 396.36 & 100.0 & 1.4 & & 1.4 & 94.4 & 1.6 & 94.4 & 321.7 & 98.4 & 34.9 & $10,864.5$ \\
\hline rc207.2 & 32 & 246.41 & 33.3 & 246.41 & 100.0 & 0.6 & & 0.6 & 98.4 & 0.7 & 96.7 & 15.1 & 97.2 & 116.4 & - \\
\hline rc208.0 & 46 & 380.56 & 8.6 & 380.56 & 100.0 & 38.1 & & 38.1 & 92.8 & 63.4 & 85.1 & $\mathrm{tl}$ & 93.4 & tl & - \\
\hline rc208.1 & 29 & 239.04 & 13.5 & 239.04 & 100.0 & 6.9 & & 6.9 & 96.5 & 0.9 & 96.1 & 34.2 & 96.1 & 990.6 & - \\
\hline rc208.2 & 31 & 213.92 & 12.7 & 213.92 & 100.0 & 1.3 & & 1.3 & 94.4 & 1.2 & 100.0 & 1.4 & 100.0 & 7.9 & - \\
\hline Avg & & & & & 99.9 & 4.2 & & 4.3 & 95.5 & 6.4 & 87.9 & 135.9 & 98.8 & 303.7 & $1,680.8$ \\
\hline Solved & & & & & & & & 27 & & 27 & & 23 & & 25 & 18 \\
\hline
\end{tabular}

The name of each instance indicates the number of vertices (excluding $p$ and $q$ ), the width of the time windows, and the instance number in the group (e.g. n20w120.1 is the first instance of the group of 5 instances with 22 vertices and time windows of 120 units). In some papers, two groups of instances (n100w80.x and n100w100.x) are included in the Dumas class instead of the Gendreau class, but the instances coincide.

Travel times and costs coincide and are first computed as truncated integer Euclidean distances and then modified to satisfy triangle inequality by iteratively setting $c_{i j}=$ $t_{i j}=t_{i k}+t_{k j}$, if $t_{i k}+t_{k j}<t_{i j}$, until no violation is identified.

Because $t$-tour relaxation requires strictly positive travel times, BMR.t was not tested on the Gendreau instances such that $t_{i j}=0$ for some $\operatorname{arc}(i, j) \in A$.

In Table 3.6, we report detailed computational results on Gendreau instances. BMR.ng and BMR.t are compared with LI. Table 3.6 shows that BMR.ng is much faster than LI and solves all 140 instances while LI solves 46 of them. BMR.t solves 18 more instances than LI. The comparison of BMR.t and LI on the instances solved by both methods shows that BMR.t outperforms LI. 
TABLE 3.5: Results on Potvin instances

\begin{tabular}{|c|c|c|c|c|c|c|c|c|c|c|c|}
\hline \multirow[b]{2}{*}{ Inst } & \multirow[b]{2}{*}{$\left|V^{\prime}\right|$} & \multirow[b]{2}{*}{$z^{*}$} & \multicolumn{6}{|c|}{ BMR.ng } & \multicolumn{2}{|c|}{ BMR.t } & \multirow{2}{*}{$\frac{\mathrm{LI}}{T_{t o t}}$} \\
\hline & & & Prec & $L B$ & $\% L B$ & $T_{L B}$ & $|\mathscr{F}|$ & $T_{t o t}$ & $\% L B$ & $T_{t o t}$ & \\
\hline rc201.1 & 21 & 444.54 & 80.0 & 444.54 & 100.0 & 0.1 & & 0.1 & 99.6 & 0.7 & 0.0 \\
\hline rc201.2 & 27 & 711.54 & 86.0 & 711.54 & 100.0 & 0.2 & & 0.2 & 100.0 & 1.3 & 0.1 \\
\hline rc201.3 & 33 & 790.61 & 86.7 & 790.61 & 100.0 & 0.2 & & 0.2 & 98.7 & 4.0 & 0.1 \\
\hline rc201.4 & 27 & 793.64 & 86.0 & 793.64 & 100.0 & 0.2 & & 0.2 & 99.4 & 3.3 & 0.0 \\
\hline rc202.1 & 34 & 771.78 & 52.2 & 771.64 & 100.0 & 1.0 & 1 & 1.3 & 92.9 & 9.0 & 164.5 \\
\hline rc202.2 & 15 & 304.14 & 33.3 & 304.14 & 100.0 & 0.2 & & 0.2 & 95.5 & 3.4 & 0.6 \\
\hline rc202.3 & 30 & 837.72 & 76.6 & 835.87 & 99.8 & 0.2 & 1 & 0.4 & 97.2 & 5.9 & 0.0 \\
\hline rc202.4 & 29 & 793.03 & 49.8 & 791.54 & 99.8 & 5.1 & 1 & 5.3 & 98.1 & 7.2 & 94.8 \\
\hline rc203.1 & 20 & 453.48 & 40.5 & 453.48 & 100.0 & 0.2 & & 0.2 & 99.4 & .2 & 2.4 \\
\hline rc203.2 & 34 & 784.16 & 32.4 & 781.64 & 99.7 & 1.7 & 1 & 2.1 & 93.4 & 11.1 & \\
\hline rc203.3 & 38 & 817.53 & 33.1 & 810.46 & 99.1 & 9.3 & 10 & 9.9 & 94.9 & 71.6 & \\
\hline rc203.4 & 16 & 314.29 & 28.3 & 314.29 & 100.0 & 0.4 & & 0.4 & 100.0 & 0.8 & 11.1 \\
\hline rc204.1 & 47 & 878.64 & 14.8 & 872.62 & 99.3 & 42.6 & 10 & 43.8 & 93.2 & $581.6^{a}$ & - \\
\hline $\mathrm{rc} 204.2$ & 34 & 662.16 & 16.2 & 650.94 & 98.3 & 21.3 & 151 & 23.1 & 90.5 & $473.4^{a}$ & \\
\hline rc204.3 & 25 & 455.03 & 17.0 & 455.03 & 100.0 & 9.9 & & 9.9 & 95.0 & 9.0 & \\
\hline rc205.1 & 15 & 343.21 & 67.6 & 343.21 & 100.0 & 0.1 & & 0.1 & 98.3 & 4.8 & 0.0 \\
\hline rc205.2 & 28 & 755.93 & 72.0 & 755.93 & 100.0 & 0.3 & & 0.3 & 98.8 & 3.7 & 0.2 \\
\hline rc205.3 & 36 & 825.06 & 57.3 & 825.06 & 100.0 & 2.2 & & 2.2 & 97.7 & 8.5 & 197.7 \\
\hline rc205.4 & 29 & 760.47 & 76.1 & 756.95 & 99.5 & 0.2 & 1 & 0.4 & 97.5 & 4.1 & 0.1 \\
\hline rc206.2 & 38 & 828.06 & 59.9 & 826.66 & 99.8 & 6.4 & 1 & 6.8 & 95.3 & 9.7 & 20.5 \\
\hline rc206.3 & 26 & 574.42 & 52.3 & 574.42 & 100.0 & 0.6 & & 0.6 & 90.0 & 6.3 & 5.5 \\
\hline rc206.4 & 39 & 831.67 & 59.0 & 827.54 & 99.5 & 1.8 & 1 & 2.2 & 90.5 & 13.4 & 69.6 \\
\hline rc207.1 & 35 & 732.68 & 42.9 & 731.57 & 99.8 & 9.0 & 1 & 9.3 & 93.3 & 11.9 & - \\
\hline rc207.2 & 32 & 701.25 & 33.7 & 694.22 & 99.0 & 53.1 & 5 & 54.8 & 89.4 & 47.2 & - \\
\hline rc207.3 & 34 & 682.40 & 30.8 & 677.23 & 99.2 & 1.9 & 1 & 2.3 & 91.8 & 15.6 & - \\
\hline rc208.1 & 39 & 789.25 & 10.4 & 785.69 & 99.5 & 46.7 & 2 & 47.5 & 90.0 & $435.9^{a}$ & - \\
\hline rc208.2 & 30 & 533.78 & 13.1 & 533.78 & 100.0 & 30.1 & & 30.1 & 93.8 & 25.2 & - \\
\hline rc208.3 & 37 & 634.44 & 10.7 & 622.48 & 98.1 & 82.0 & 24 & 86.8 & 94.1 & $532.6^{a}$ & \\
\hline Avg & & & & & 99.7 & 11.7 & & 12.2 & 95.3 & 11.7 & 33.3 \\
\hline Solved & & & & & & & & 28 & & 24 & 17 \\
\hline
\end{tabular}

${ }^{a}$ BMR.t runs out of memory

\subsubsection{Olhmann Instances}

The Olhmann class was proposed by Ohlmann and Thomas [2007] and consists of 25 instances divided in five groups. The instances have 152 or 202 vertices and are obtained from the Dumas instances by extending the time windows by 100 time units. Travel times and travel costs are computed as for the Gendreau class.

To our knowledge, no exact method has been tested on this class so far. In Table 3.7, we report the computational results of BMR.ng. BMR.t was not tested on these instances as the requirement of strictly positive travel times is not satisfied by any of the instances.

The table shows that all but one instance can be solved in a reasonable amount of computing time. Instance n200w140.4 could not be solved as BMR.ng ran out of memory (i.e., $|\mathscr{F}|>10^{8}$ ). BMR.ng ran out of memory after 2,413.1 seconds at iteration 
TABLE 3.6: Results on Gendreau instances

\begin{tabular}{|c|c|c|c|c|c|c|c|c|c|c|c|c|c|c|c|}
\hline \multirow[b]{2}{*}{ Inst } & \multirow[b]{2}{*}{$z^{*}$} & \multicolumn{3}{|c|}{ BMR.ng } & \multicolumn{2}{|c|}{ BMR.t } & \multirow{2}{*}{$\frac{\mathrm{LI} \mid}{T_{t o t} \mid}$} & & & & BMR.n & & $\mathrm{BM}$ & IR.t & LI \\
\hline & & Prec & $\% L B$ & $T_{\text {tot }}$ & $\% L B$ & $T_{\text {tot }}$ & & Inst & $z^{*}$ & Prec & $\% L B$ & $T_{\text {tot }}$ & $\% L B$ & $T_{\text {tot }}$ & $T_{\text {tot }}$ \\
\hline $\mathrm{n} 20 \mathrm{w} 120.1$ & 267 & 62.8 & 100.0 & 0.4 & 100.0 & 0.2 & 0.2 & n60w200.1 & 410 & 47.2 & 100.0 & 14.7 & 89.2 & $714.0^{b}$ & - \\
\hline $\mathrm{n} 20 \mathrm{w} 120.2$ & 218 & 52.4 & 100.0 & 0.5 & 96.6 & 0.2 & 0.3 & n60w200.2 & 414 & 52.1 & 100.0 & 7.4 & $a$ & & - \\
\hline $\mathrm{n} 20 \mathrm{w} 120.3$ & 303 & 63.2 & 100.0 & 0.5 & 94.2 & 0.2 & 0.1 & n60w200.3 & 455 & 46.1 & 97.6 & 192.1 & $a$ & & - \\
\hline n20w120.4 & 300 & 63.2 & 100.0 & 0.3 & 97.2 & 0.1 & 0.1 & n60w200.4 & 431 & 52.2 & 99.7 & 18.4 & 96.6 & 14.7 & - \\
\hline n20w120.5 & 240 & 60.2 & 100.0 & 0.3 & 99.3 & 0.3 & 0.3 & n60w200.5 & 427 & 47.5 & 99.6 & 20.0 & $a$ & & - \\
\hline n20w140.1 & 176 & 50.2 & 100.0 & 0.4 & 95.3 & 0.6 & 0.5 & $\mathrm{n} 80 \mathrm{w} 100.1$ & 541 & 74.2 & 100.0 & 3.8 & $a$ & & - \\
\hline $\mathrm{n} 20 \mathrm{w} 140.2$ & 272 & 56.7 & 100.0 & 0.4 & 95.9 & 0.3 & 0.1 & $\mathrm{n} 80 \mathrm{w} 100.2$ & 567 & 80.4 & 100.0 & 1.1 & $a$ & & - \\
\hline $\mathrm{n} 20 \mathrm{w} 140.3$ & 236 & 54.5 & 100.0 & 0.3 & 92.9 & 0.5 & 0.1 & n80w100.3 & 578 & 75.7 & 100.0 & 3.4 & $a$ & & - \\
\hline $\mathrm{n} 20 \mathrm{w} 140.4$ & 255 & 51.1 & 100.0 & 1.2 & 96.3 & 0.5 & 0.3 & $\mathrm{n} 80 \mathrm{w} 100.4$ & 648 & 82.0 & 99.6 & 20.4 & 98.3 & 1.2 & - \\
\hline n20w140.5 & 225 & 58.9 & 100.0 & 0.3 & 88.4 & 0.6 & 0.1 & n80w100.5 & 532 & 76.4 & 100.0 & 6.1 & $a$ & & - \\
\hline n20w160.1 & 241 & 51.9 & 100.0 & 0.6 & 92.5 & 0.4 & 1.6 & |n80w120.1 & 498 & 70.8 & 99.9 & 27.0 & $a$ & & - \\
\hline $\mathrm{n} 20 \mathrm{w} 160.2$ & 201 & 52.8 & 100.0 & 0.3 & 95.0 & 0.2 & 0.1 & $\mathrm{n} 80 \mathrm{w} 120.2$ & 577 & 73.6 & 99.9 & 16.0 & $a$ & & - \\
\hline n20w160.3 & 201 & 60.2 & 100.0 & 0.3 & 98.5 & 0.3 & 0.1 & $\mathrm{n} 80 \mathrm{w} 120.3$ & 540 & 73.1 & 100.0 & 6.4 & $a$ & & - \\
\hline $\mathrm{n} 20 \mathrm{w} 160.4$ & 203 & 42.0 & 100.0 & 0.7 & 90.8 & 0.4 & 3.4 & n80w120.4 & 501 & 68.7 & 98.1 & 61.4 & $a$ & & - \\
\hline n20w160.5 & 245 & 50.6 & 100.0 & 0.3 & 87.7 & 0.7 & 0.3 & n80w120.5 & 591 & 72.2 & 99.3 & 39.6 & $a$ & & - \\
\hline $\mathrm{n} 20 \mathrm{w} 180.1$ & 253 & 51.5 & 100.0 & 0.3 & 97.7 & 0.3 & 0.4 & |n80w140.1 & 511 & 68.0 & 100.0 & 7.5 & $a$ & & - \\
\hline $\mathrm{n} 20 \mathrm{w} 180.2$ & 265 & 50.2 & 100.0 & 0.3 & 99.4 & 0.4 & 0.3 & n80w140.2 & 470 & 67.8 & 99.8 & 77.8 & $a$ & & - \\
\hline n20w180.3 & 271 & 44.2 & 100.0 & 1.0 & 94.1 & 0.5 & 0.4 & n80w140.3 & 580 & 68.7 & 99.1 & 46.5 & $a$ & & - \\
\hline n20w180.4 & 201 & 42.9 & 100.0 & 0.4 & 96.4 & 0.3 & 3.2 & n80w140.4 & 422 & 67.8 & 99.6 & 38.8 & $a$ & & - \\
\hline n20w180.5 & 193 & 35.9 & 100.0 & 0.3 & 91.7 & 0.7 & 27.2 & n80w140.5 & 545 & 68.4 & 100.0 & 8.1 & 94.5 & 282.7 & - \\
\hline n20w200.1 & 233 & 38.1 & 100.0 & 1.1 & 93.1 & 0.4 & 12.0 & n80w160.1 & 506 & 62.6 & 100.0 & 93.7 & $a$ & & - \\
\hline $\mathrm{n} 20 \mathrm{w} 200.2$ & 203 & 31.6 & 100.0 & 1.1 & 94.9 & 0.6 & 55.7 & n80w160.2 & 548 & 60.6 & 99.9 & 194.8 & $a$ & & - \\
\hline n20w200.3 & 249 & 40.3 & 100.0 & 0.8 & 89.5 & 0.8 & 4.5 & $\mathrm{n} 80 \mathrm{w} 160.3$ & 521 & 60.4 & 100.0 & 58.2 & $a$ & & - \\
\hline n20w200.4 & 293 & 41.6 & 98.8 & 2.4 & 90.6 & 0.8 & 3.0 & $\mathrm{n} 80 \mathrm{w} 160.4$ & 509 & 63.5 & 100.0 & 50.8 & $a$ & & - \\
\hline n20w200.5 & 227 & 34.2 & 100.0 & 0.4 & 99.5 & 0.3 & 26.4 & n80w160.5 & 438 & 63.3 & 100.0 & 58.1 & 92.8 & $576.8^{b}$ & - \\
\hline n40w120.1 & 434 & 70.4 & 100.0 & 2.1 & $a$ & & 15.7 & n80w180.1 & 551 & 57.8 & 99.9 & 75.4 & 91.9 & $702.3^{b}$ & - \\
\hline $\mathrm{n} 40 \mathrm{w} 120.2$ & 444 & 66.7 & 100.0 & 1.9 & 95.4 & 1.7 & 51.5 & $\mathrm{n} 80 \mathrm{w} 180.2$ & 478 & 58.5 & 100.0 & 61.5 & $a$ & & - \\
\hline $\mathrm{n} 40 \mathrm{w} 120.3$ & 357 & 65.9 & 100.0 & 2.1 & 98.8 & 1.5 & 39.1 & $\mathrm{n} 80 \mathrm{w} 180.3$ & 524 & 55.3 & 100.0 & 84.3 & 89.7 & $967.6^{b}$ & - \\
\hline $\mathrm{n} 40 \mathrm{w} 120.4$ & 303 & 63.8 & 100.0 & 1.9 & 88.7 & 2.4 & 28.6 & n80w180.4 & 479 & 59.6 & 100.0 & 47.4 & $a$ & & - \\
\hline n40w120.5 & 350 & 68.3 & 100.0 & 1.3 & 98.9 & 0.9 & 2.4 & n80w180.5 & 470 & 61.5 & 100.0 & 49.0 & 91.9 & $602.8^{b}$ & - \\
\hline n40w140.1 & 328 & 65.2 & 100.0 & 2.0 & 97.3 & 1.0 & 42.9 & |n80w200.1 & 490 & 47.5 & 99.3 & 177.4 & $a$ & & - \\
\hline $\mathrm{n} 40 \mathrm{w} 140.2$ & 383 & 63.8 & 100.0 & 1.5 & 92.4 & 3.2 & 83.2 & n80w200.2 & 488 & 55.1 & 99.9 & 32.1 & $a$ & & - \\
\hline $\mathrm{n} 40 \mathrm{w} 140.3$ & 398 & 66.9 & 100.0 & 2.2 & $a$ & & 5.5 & n80w200.3 & 464 & 50.0 & 99.6 & 39.5 & $a$ & & - \\
\hline n40w140.4 & 342 & 59.9 & 100.0 & 1.7 & 93.1 & 2.1 & 230.5 & $\mathrm{n} 80 \mathrm{w} 200.4$ & 526 & 54.1 & 99.1 & 63.3 & $a$ & & - \\
\hline $\mathrm{n} 40 \mathrm{w} 140.5$ & 371 & 56.3 & 100.0 & 6.2 & 95.7 & 1.5 & $1,288.1$ & n80w200.5 & 439 & 49.0 & 99.8 & 46.9 & $a$ & & - \\
\hline n40w160.1 & 348 & 56.8 & 100.0 & 1.4 & 94.3 & 1.0 & 117.3 & n100w 80.1 & 670 & 84.3 & 100.0 & 4.5 & $a$ & & - \\
\hline n40w160.2 & 337 & 56.1 & 100.0 & 1.2 & 95.7 & 0.9 & $1,331.4$ & n100w80.2 & 666 & 86.4 & 99.9 & 2.5 & 98.3 & 2.9 & - \\
\hline n40w160.3 & 346 & 53.7 & 100.0 & 3.5 & $a$ & & 474.4 & n100w80.3 & 691 & 85.4 & 100.0 & 1.4 & 97.9 & 7.0 & - \\
\hline n40w160.4 & 288 & 47.5 & 100.0 & 12.9 & 90.6 & 196.6 & - & n100w80.4 & 700 & 84.1 & 100.0 & 2.8 & $a$ & & - \\
\hline n40w160.5 & 315 & 51.5 & 100.0 & 6.9 & 92.6 & 4.5 & $9,369.0$ & n100w80.5 & 603 & 82.3 & 100.0 & 2.6 & $a$ & & - \\
\hline n40w180.1 & 337 & 51.3 & 100.0 & 25.9 & 87.7 & 9.7 & - & $\mathrm{n} 100 \mathrm{w} 100.1$ & 643 & 79.7 & 100.0 & 18.4 & $a$ & & - \\
\hline $\mathrm{n} 40 \mathrm{w} 180.2$ & 347 & 47.5 & 100.0 & 14.9 & $a$ & & - & $\mathrm{n} 100 \mathrm{w} 100.2$ & 618 & 81.0 & 100.0 & 2.5 & 96.3 & 37.3 & - \\
\hline n40w180.3 & 279 & 51.7 & 100.0 & 1.6 & $a$ & & - & n100w100.3 & 685 & 80.5 & 100.0 & 4.5 & 96.3 & 15.8 & - \\
\hline $\mathrm{n} 40 \mathrm{w} 180.4$ & 354 & 52.7 & 99.6 & 7.5 & 96.6 & 1.1 & - & n100w100.4 & 684 & 79.9 & 99.5 & 83.6 & $a$ & & - \\
\hline $\mathrm{n} 40 \mathrm{w} 180.5$ & 335 & 43.9 & 100.0 & 4.8 & 86.2 & 48.0 & -1 & n100w100.5 & $\mathbf{5 7 2}$ & 78.2 & 100.0 & 18.6 & $a$ & & - \\
\hline n40w200.1 & 330 & 39.7 & 100.0 & 8.8 & $a$ & & -1 & n100w120.1 & 629 & 78.2 & 100.0 & 34.5 & $a$ & & - \\
\hline $\mathrm{n} 40 \mathrm{w} 200.2$ & 303 & 39.6 & 99.7 & 17.1 & $a$ & & - & $\mathrm{n} 100 \mathrm{w} 120.2$ & 540 & 77.4 & 99.2 & 24.1 & $a$ & & - \\
\hline n40w200.3 & 339 & 42.0 & 95.0 & 25.8 & $a$ & & - & $\mathrm{n} 100 \mathrm{w} 120.3$ & 615 & 74.2 & 100.0 & 61.6 & 97.1 & 33.2 & - \\
\hline n40w200.4 & 301 & 47.6 & 99.7 & 12.9 & 87.4 & 12.5 & - & n100w120.4 & 662 & 79.7 & 99.7 & 91.4 & $a$ & & - \\
\hline n40w200.5 & 296 & 35.0 & 100.0 & 8.9 & 93.0 & 4.6 & -1 & n100w120.5 & 537 & 74.9 & 100.0 & 42.1 & $a$ & & - \\
\hline n60w120.1 & 384 & 69.7 & 100.0 & 17.6 & 96.1 & 3.0 & $8,431.4$ & n100w140.1 & 603 & 71.2 & 100.0 & 38.1 & $a$ & & - \\
\hline n60w120.2 & 426 & 72.6 & 100.0 & 4.1 & 99.0 & 0.9 & 419.3 & 140.2 & 613 & 74.3 & 100.0 & 44.4 & $a$ & & - \\
\hline n60w120.3 & 407 & 69.1 & 100.0 & 6.0 & $a$ & & $20,411.3$ & n100w140.3 & 481 & 73.9 & 100.0 & 51.5 & $a$ & & - \\
\hline n60w120.4 & 490 & 70.1 & 100.0 & 4.8 & 96.5 & 5.0 & $13,702.2$ & n100w140.4 & 533 & 74.3 & 100.0 & 24.7 & $a$ & & - \\
\hline n60w120.5 & 547 & 70.7 & 100.0 & 9.1 & 96.9 & 1.9 & $1,455.1$ & n100w140.5 & 509 & 73.1 & 99.1 & 66.9 & 90.5 & $725.4^{b}$ & - \\
\hline n60w140.1 & 423 & 65.8 & 99.6 & 8.4 & $a$ & & - & n100w160.1 & 582 & 69.4 & 100.0 & 117.4 & 92.0 & $310.0^{b}$ & - \\
\hline n60w140.2 & 462 & 67.3 & 99.8 & 11.5 & 96.0 & 2.8 & $4,497.5$ & $\mathrm{n} 100 \mathrm{w} 160.2$ & 530 & 68.8 & 99.8 & 99.5 & $a$ & & - \\
\hline n60w140.3 & 427 & 66.0 & 100.0 & 11.8 & 94.6 & 10.1 & 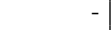 & n100w160.3 & 495 & 71.5 & 100.0 & 52.6 & $a$ & & - \\
\hline n60w140.4 & 488 & 67.6 & 100.0 & 14.2 & 93.7 & 2.9 & - & n100w160.4 & 580 & 69.8 & 100.0 & 116.6 & $a$ & & - \\
\hline n60w140.5 & 460 & 66.1 & 99.0 & 33.4 & 90.6 & 8.5 & $5,148.5$ & n100w160.5 & 586 & 71.9 & 99.9 & 72.9 & $a$ & & - \\
\hline n60w160.1 & 560 & 63.5 & 99.3 & 42.6 & 90.9 & 458.2 & -1 & |n100w180.1 & 568 & 60.6 & 99.4 & 170.4 & $a$ & & - \\
\hline n60w160.2 & 423 & 62.3 & 100.0 & 7.7 & 98.7 & & - & $\mathrm{n} 100 \mathrm{w} 180.2$ & 503 & 60.8 & 100.0 & 40.4 & $a$ & & - \\
\hline n60w160.3 & 434 & 54.5 & & 34.8 & 92.8 & $184.0^{b}$ & - & n100w180.3 & 574 & 61.9 & 99.0 & & $a$ & & - \\
\hline n60w160.4 & 401 & 62.7 & 100.0 & 4.0 & 96.5 & 6.2 & - & $\mathrm{n} 100 \mathrm{w} 180.4$ & 526 & 61.7 & 100.0 & 128.4 & $a$ & & - \\
\hline n60w160.5 & 501 & 63.0 & 100.0 & 7.2 & 91.6 & 356.0 & -1 & n100w180.5 & 501 & 60.0 & 98.6 & 117.9 & $a$ & & - \\
\hline n60w180.1 & 411 & 56.7 & 99.1 & 103.9 & $a$ & & -1 & n100w200.1 & 549 & 55.7 & 100.0 & 424.3 & $a$ & & - \\
\hline n60w180.2 & 399 & 53.7 & 100.0 & 8.4 & 95.4 & 13.3 & - & n100w200.2 & 502 & 55.9 & 100.0 & 86.9 & $a$ & & - \\
\hline n60w180.3 & 444 & 54.7 & 99.1 & 28.5 & 90.9 & $624.0^{b}$ & - & n100w200.3 & 557 & 57.9 & 99.5 & 264.6 & $a$ & & - \\
\hline n60w 180.4 & 456 & 54.9 & 99.9 & 51.6 & 93.5 & 20.8 & - & n100w200.4 & 521 & 57.3 & 99.4 & 199.5 & $a$ & & - \\
\hline n60w180.5 & 395 & 50.3 & 98.4 & 39.3 & $a$ & & -1 & n100w200.5 & 486 & 55.5 & 99.8 & 104.7 & $a$ & & - \\
\hline & & & & & & & & $\begin{array}{l}\text { Avg } \\
\text { Solved }\end{array}$ & & & 99.8 & $\begin{array}{r}36.7 \\
140\end{array}$ & 94.2 & $\begin{array}{r}24.9 \\
64\end{array}$ & $\begin{array}{r}1,462.7 \\
46\end{array}$ \\
\hline
\end{tabular}

$b$ BMR.t runs out of memory 
TABLE 3.7: Results on Olhmann instances

\begin{tabular}{|c|c|c|c|c|c|c|c|}
\hline \multirow[b]{2}{*}{ Inst } & \multirow[b]{2}{*}{$z^{*}$} & \multicolumn{6}{|c|}{ BMR.ng } \\
\hline & & Prec & $L B$ & $\% L B$ & $T_{L B}$ & $|\mathscr{F}|$ & $T_{t o t}$ \\
\hline n150w120.1 & 732 & 77.5 & 725.5 & 99.1 & 243.8 & 243 & 248.6 \\
\hline n150w120.2 & 677 & 77.8 & 668.4 & 98.7 & 130.5 & 12,149 & 260.6 \\
\hline n150w120.3 & 747 & 76.5 & 746.4 & 99.9 & 160.0 & 2 & 163.2 \\
\hline n150w120.4 & 762 & 78.0 & 761.6 & 99.9 & 172.8 & 1 & 176.0 \\
\hline n150w120.5 & 689 & 76.9 & 684.7 & 99.4 & 113.1 & 373 & 118.9 \\
\hline n150w140.1 & 762 & 72.5 & 754.0 & 98.9 & 198.2 & 14,993 & 350.2 \\
\hline n150w140.2 & 753 & 74.5 & 752.0 & 99.9 & 439.0 & 7 & 442.1 \\
\hline n150w140.3 & 613 & 73.3 & 608.5 & 99.3 & 420.6 & 1,671 & 440.9 \\
\hline n150w140.4 & 676 & 73.7 & 676.0 & 100.0 & 104.8 & & 104.8 \\
\hline n150w140.5 & 663 & 73.1 & 662.0 & 99.8 & 365.9 & 3 & 369.4 \\
\hline n150w160.1 & 704 & 70.4 & 701.4 & 99.6 & 262.9 & 48 & 266.5 \\
\hline n150w160.2 & 711 & 69.3 & 709.7 & 99.8 & 213.1 & 5 & 216.4 \\
\hline n150w160.3 & 608 & 71.9 & 603.2 & 99.2 & 483.0 & 352 & 491.8 \\
\hline n150w160.4 & 672 & 69.9 & 672.0 & 100.0 & 570.6 & 12 & 577.1 \\
\hline n150w160.5 & 658 & 71.0 & 655.0 & 99.5 & 169.5 & 44 & 173.4 \\
\hline n200w120.1 & 795 & 78.3 & 793.3 & 99.8 & 303.8 & 57 & 308.8 \\
\hline n200w120.2 & 721 & 80.2 & 713.9 & 99.0 & 110.4 & 20,223 & 257.1 \\
\hline n200w120.3 & 879 & 80.4 & 868.6 & 98.8 & 151.1 & 57,625 & 958.6 \\
\hline n200w120.4 & 777 & 79.1 & 775.8 & 99.8 & 412.3 & 13 & 417.1 \\
\hline n200w120.5 & 840 & 79.6 & 833.2 & 99.2 & 237.4 & 29,072 & 574.3 \\
\hline n200w140.1 & 830 & 77.1 & 826.2 & 99.5 & $1,217.8$ & 6,261 & $1,274.9$ \\
\hline n200w140.2 & 760 & 76.3 & 756.2 & 99.5 & 614.5 & 407 & 624.5 \\
\hline n200w140.3 & 758 & 76.3 & 756.0 & 99.7 & 250.7 & 133 & 259.0 \\
\hline n200w140.4 & $816^{a}$ & 75.3 & 807.1 & 98.9 & 592.9 & - & $2,413.1^{b}$ \\
\hline n200w140.5 & 822 & 76.6 & 819.6 & 99.7 & 515.2 & 90 & 521.0 \\
\hline Avg & & & & 99.5 & 338.2 & & 399.8 \\
\hline Solved & & & & & & & 24 \\
\hline
\end{tabular}

$h=7$ with $z_{U B}^{7}=814$. Thus, $z_{U B}^{6}=813$ is a valid lower bound on the optimal solution cost.

\subsubsection{More Details on the Computational Results}

Table 3.8 shows average results of the exact method, BMR.ng, using either the $n g$-tour or $n g L$-tour relaxations on the 6 classes of instances. Columns $\% L B$ show that the $n g L$-tour relaxation closes half of the gap left by the $n g$-tour relaxation but is more time-consuming. Columns "Solved" show the effectiveness of the $n g L$-tour relaxation in solving the Ohlmann instances. BMR.ng using the $n g$-tour relaxation cannot solve 4 of the instances $(n 150 w 120.2, n 150 w 140.1, n 200 w 120.5, n 200 w 140.1)$ solved by using the $n g L$-tour relaxation.

Table 3.9 shows the effectiveness of the dominance and fathoming rules applied in Step 2 of the exact method (see $§ 3.3$ ). For a selected set of difficult instances, the table 
TABLE 3.8: Comparison of $n g$-tour and $n g L$-tour relaxations

\begin{tabular}{|c|c|c|c|c|c|c|c|c|c|}
\hline \multirow[b]{2}{*}{ Class } & \multirow[b]{2}{*}{ \#instances } & \multicolumn{4}{|c|}{ BMR.ng with $n g$-tour } & \multicolumn{4}{|c|}{ BMR.ng with $n g L$-tour } \\
\hline & & Solved & $\% L B$ & $T_{L B}$ & $T_{\text {tot }}$ & Solved & $\% L B$ & $T_{L B}$ & $T_{\text {tot }}$ \\
\hline Ascheuer Easy & 32 & 32 & 99.00 & 1.4 & 1.7 & 32 & 99.99 & 2.6 & 2.8 \\
\hline Ascheuer Hard & 18 & 18 & 99.60 & 23.5 & 33.4 & 18 & 99.70 & 89.2 & 114.1 \\
\hline Pesant & 27 & 27 & 99.95 & 3.1 & 3.3 & 27 & 99.95 & 4.2 & 4.3 \\
\hline Potvin & 28 & 28 & 99.50 & 8.9 & 9.7 & 28 & 99.67 & 11.7 & 12.2 \\
\hline Gendreau & 140 & 140 & 99.59 & 19.1 & 37.7 & 140 & 99.78 & 35.6 & 36.9 \\
\hline Olhmann & 25 & 20 & 99.25 & 197.1 & 242.6 & 24 & 99.55 & 340.1 & 404.7 \\
\hline Avg & & & 99.52 & 28.0 & 42.1 & & 99.78 & 56.8 & 65.0 \\
\hline Sum & 270 & 265 & & & & 269 & & & \\
\hline
\end{tabular}

TABLE 3.9: Effectiveness of the Dominance and Fathoming Rules

\begin{tabular}{|c|c|c|c|c|c|c|c|c|}
\hline \multirow[b]{2}{*}{ Inst } & \multicolumn{2}{|c|}{ Fath 1} & \multicolumn{2}{|c|}{ Dom 1} & \multicolumn{2}{|c|}{ Fath 2} & \multicolumn{2}{|c|}{ Undominated } \\
\hline & $n_{1}$ & $\% n_{1}$ & $n_{2}$ & $\% n_{2}$ & $n_{3}$ & $\% n_{3}$ & $n_{4}$ & $\% n_{4}$ \\
\hline rbg049a & 71 & 54.7 & 4 & 5.4 & 50 & 89.4 & 6 & 4.5 \\
\hline rbg050b & 13 & 40.2 & 1 & 2.5 & 18 & 92.9 & 2 & 4.1 \\
\hline rbg050c & 1,275 & 52.5 & 80 & 6.9 & 979 & 91.3 & 93 & 3.8 \\
\hline $\operatorname{rbg} 193.2$ & 6,394 & 70.6 & 1,027 & 38.6 & 1,219 & 74.6 & 414 & 4.6 \\
\hline rbg233.2 & 166,371 & 75.0 & 24,763 & 44.7 & 21,671 & 70.7 & 8,969 & 4.0 \\
\hline n150w120.2 & 369,823 & 76.5 & 6,281 & 5.5 & 95,468 & 88.7 & 12,149 & 2.5 \\
\hline n150w140.1 & 579,249 & 79.9 & 3,506 & 2.4 & 126,949 & 89.4 & 14,993 & 2.1 \\
\hline n200w120.2 & 629,067 & 77.7 & 5,461 & 3.0 & 154,832 & 88.4 & 20,223 & 2.5 \\
\hline n200w120.3 & $2,338,415$ & 80.5 & 23,374 & 4.1 & 486,025 & 89.4 & 57,625 & 2.0 \\
\hline n200w120.5 & $1,389,485$ & 81.8 & 9,951 & 3.2 & 270,429 & 90.3 & 29,072 & 1.7 \\
\hline n200w140.1 & 320,984 & 82.8 & 1,673 & 2.5 & 58,525 & 90.3 & 6,261 & 1.6 \\
\hline & & & & & & & & \\
\hline
\end{tabular}

reports the following columns: the number of states fathomed by Fathoming $1\left(n_{1}\right)$, the percentage of times Fathoming 1 is successfully applied $\left(\% n_{1}\right)$, the number of states dominated by Dominance $1\left(n_{2}\right)$, the percentage of times Dominance 1 is successfully applied $\left(\% n_{2}\right)$, the number of states fathomed by Fathoming $2\left(n_{3}\right)$, the percentage of times Fathoming 2 is successfully applied $\left(\% n_{3}\right)$, the number of undominated states $\left(n_{4}\right)$, and the percentage of undominated states on the number of states generated $\left(\% n_{4}\right)$.

From Table 3.9, the effectiveness of the dominance and fathoming rules is noticeable. In particular, it is remarkable that 70 to $90 \%$ of the states that are not discarded by Fathoming 1 and Dominance 1 can be fathomed with Fathoming 2. 


\subsection{Conclusions}

In this chapter, we described an exact dynamic programming algorithm for the Traveling Salesman Problem with Time Windows (TSPTW) based on two tour relaxations called $n g$-tour and $n g L$-tour relaxations.

We reported extensive computational results on several classes of both real-world and random instances taken from the literature and used them to test both exact and heuristic methods involving up to 233 vertices.

The exact algorithm solved all but one instance and outperforms all previously published exact methods for the TSPTW, solving 136 out of 270 instances for the first time. 



\section{Chapter 4}

\section{Vehicle Routing Problem with Time Windows}

1

In this chapter, we describe an effective exact method for solving both the capacitated vehicle routing problem (CVRP) and the vehicle routing problem with time windows (VRPTW) that improves the method proposed by Baldacci et al. [2008] for the CVRP. The proposed algorithm is based on the set partitioning formulation of the problem. The $n g$-route relaxation, introduced in Chapter 3 , is used by different dual-ascent heuristics to find near-optimal dual solutions of the linear relaxation of the set partitioning model. We describe a column-and-cut generation algorithm strengthened by valid inequalities that uses a new strategy for solving the pricing problem. The $n g$-route relaxation and the different dual solutions achieved allow us to generate a reduced set partitioning problem containing all routes of any optimal solution that is finally solved by an integer programming solver. The proposed method solves 4 of the 5 open Solomon VRPTW instances and significantly improves the running times of state-of-the-art algorithms for both VRPTW and CVRP.

\subsection{Introduction}

The problem of supplying customers with vehicles based at a central depot is generally known as vehicle routing problem (VRP). The solution of a VRP calls for the design of a set of routes, each performed by a vehicle that starts and ends at the depot, such that all customers are serviced, a set of operational constraints are satisfied, and the total routing cost is minimized.

\footnotetext{
${ }^{1}$ This chapter is based on Baldacci et al. [2010, 2011a, 2012a]
} 
The two most studied members of the VRP family are the capacitated vehicle routing problem (CVRP) and the vehicle routing problem with time windows (VRPTW). In the CVRP, a fleet of identical capacitated vehicles located at a central depot must be optimally routed to supply a set of customers with known demands. Each vehicle can perform at most one route, and the total demand delivered by a route cannot exceed the vehicle capacity. The VRPTW generalizes the CVRP by imposing that each customer must be visited within a specified time interval, called time window. The CVRP is $\mathcal{N} \mathcal{P}$-hard, so is the VRPTW.

\subsection{Literature Review}

The most effective exact algorithms for the CVRP are owed to Baldacci et al. [2004], Lysgaard et al. [2004], Fukasawa et al. [2006], and Baldacci et al. [2008]. Baldacci et al. [2004] described a branch-and-cut (BC) algorithm based on a two-commodity network flow formulation of the problem. Lysgaard et al. [2004] proposed a BC algorithm that enhances the method of Augerat et al. [1995]. The method of Fukasawa et al. [2006] combines a BC based on the algorithm of Lysgaard et al. [2004] with a new branchand-cut-and-price (BCP) algorithm based on the two-index and the set partitioning (SP) formulations. The lower bound is computed with a column-and-cut generation method that uses $k$-cycle-free $q$-routes (with $k$ up to 4 ) instead of feasible CVRP routes and the valid inequalities used by Lysgaard et al. [2004]. The algorithm decides at the root node to use either a pure BC or the BCP algorithm. Baldacci et al. [2008] presented an algorithm based on the SP model strengthened with capacity and clique inequalities and were the first to compute lower bounds based on elementary routes. Their algorithm could not solve three instances solved by Fukasawa et al. [2006] but is faster with regards to the problems solved by both methods.

The first exact algorithm for the VRPTW based on the SP formulation was the branchand-price (BP) algorithm of Desrochers et al. [1992]. This method was improved by Kohl et al. [1999] by adding 2-path inequalities to linear relaxation of the SP formulation. Kohl and Madsen [1997] proposed a branch-and-bound algorithm where the lower bounds were computed using subgradient and bundle methods. These methods were based on 2-cycle elimination algorithms. Irnich and Villeneuve [2006] described a $\mathrm{BP}$ algorithm where the pricing subproblem is solved with a $k$-cycle elimination procedure. Algorithms based on elementary routes were proposed by Feillet et al. [2004] and Chabrier [2006]. Jepsen et al. [2008] described a BCP algorithm based on the SP model and Subset-Row (SR) inequalities. This method was improved by Desaulniers et al. [2008] by adding both SR and generalized $k$-path inequalities and by using a tabu search heuristic, instead of dynamic programming (DP) algorithms, to rapidly generate negative reduced cost routes. Their method outperforms all other algorithms, 
remarkably decreasing the computational time, and solving 5 of the 10 open Solomon instances.

In general, any exact algorithm for the VRPTW based on the SP model can be easily adapted to solve the CVRP by simply relaxing the time window constraints in the pricing algorithm. Nonetheless, such a simple adaptation might not be effective, and none of the methods published in the literature so far for the VRPTW have been proven to effectively solve the CVRP.

The method of Baldacci et al. [2008] for the CVRP is based on an exact solution framework that can be tailored to solve the VRPTW as well as several other variants of the VRP. The method has the following steps: (i) use different bounding procedures to find near-optimal dual solutions of the linear relaxation of the SP model strengthened by valid inequalities; (ii) use a column-and-cut generation procedure to reduce the integrality gap by adding, in a cutting plane fashion, both strengthened capacity and clique constraints; (iii) use the final dual solution achieved at Step (ii) to generate a reduced SP problem containing only the routes of reduced cost less than or equal to the gap between a known upper bound and the lower bound obtained; (iv) solve the resulting reduced problem with an integer programming (IP) solver.

The key components of this method are the bounding procedures of Step (i) that are based on the state-space relaxation technique, introduced in Christofides et al. [1981c], to extend the route set with a relaxation of feasible routes easier to compute, and the use of bounding functions to reduce the state-space graph computed by DP when solving the pricing problem and generating the final SP model.

In this chapter, we describe an exact method to solve both the VRPTW and the CVRP that improves the method of Baldacci et al. [2008]. We show that the $n g$-route relaxation (described in Chapter 3 for the TSPTW) improves other nonelementary route relaxations proposed for the CVRP and VRPTW. This relaxation is particularly effective for difficult VRPTW instances with wide time windows and loose capacity constraints. The $n g$-route relaxation is used at Step (i) to derive a new dual-ascent heuristic and at Steps (ii) and (iii) to reduce the state-space graph of the DP algorithm in generating elementary routes. We also describe a new family of valid inequalities, called weak subset-row inequalities, that are a relaxation of SR inequalities. The main advantage of these new inequalities is that their duals can be implicitly considered while solving the pricing problem. Moreover, we propose new ideas to improve the pricing algorithm in the column-and-cut procedure based on the use of the dual solution achieved at Step (i) to eliminate routes of negative reduced costs with respect to the current dual solution that cannot be in any optimal solution. Finally, we report computational results for both VRPTW and CVRP showing that the proposed method solves 4 of the 5 open Solomon VRPTW instances and significantly improves the running times of state-of-the-art algorithms for both VRPTW and CVRP. 


\subsection{Mathematical Formulation and its Relaxations}

The VRPTW is defined on a complete digraph $G=\left(V^{\prime}, A\right)$, where $V^{\prime}=\{0,1, \ldots, n\}$ is a set of $n+1$ vertices and $A$ is the arc set. Vertex 0 represents the depot, and the vertex subset $V=V^{\prime} \backslash\{0\}$ corresponds to $n$ customers. A demand $q_{i}$ and a time window $\left[e_{i}, l_{i}\right]$ are associated with each vertex $i \in V^{\prime}$, where $e_{i}$ and $l_{i}$ represent the earliest and the latest time to visit vertex $i$. Time windows are "hard", meaning that if a vehicle visits a customer before time $e_{i}$, the service is postponed until time $e_{i}$. We assume that $q_{0}=0$. A travel cost $d_{i j}$ and a travel time $t_{i j}>0$ are associated with each $\operatorname{arc}(i, j) \in A$; without loss of generality, we assume that travel time $t_{i j}$ includes the service time at vertex $i$, so the departure time from any customer $i \in V$ coincides with the end of its service. We also assume that matrices $d_{i j}$ and $t_{i j}$ satisfy the triangle inequality; therefore, time windows, travel times, and customer demands can be used to properly reduce the arc set $A$ as described in Desrochers et al. [1992]. For each vertex $i \in V^{\prime}$, we indicate with $\Gamma_{i} \subseteq V^{\prime}$ the set of successors of vertex $i$ in graph $G$ (i.e., $\left.\Gamma_{i}=\left\{j \in V^{\prime}:(i, j) \in A\right\}\right)$ and with $\Gamma_{i}^{-1} \subseteq V^{\prime}$ the set of predecessors of vertex $i$ in graph $G$ (i.e., $\left.\Gamma_{i}^{-1}=\left\{j \in V^{\prime}:(j, i) \in A\right\}\right)$.

A fleet of $m$ identical vehicles of capacity $Q$ stationed at the depot must fulfill customer demands. A vehicle route $R=\left(0, i_{1}, \ldots, i_{r}, 0\right)$, with $r \geq 1$, is a simple circuit in graph $G$ that passes through the depot, visits vertices $V(R)=\left\{0, i_{1}, \ldots, i_{r}\right\} \subseteq V^{\prime}$, within their time windows, leaves the depot 0 at time $e_{0}$, returns to the depot 0 before time $l_{0}$, and such that the total demand of visited customers does not exceed the vehicle capacity $Q$ (i.e., $\left.\sum_{i \in V(R)} q_{i} \leq Q\right)$. The cost of route $R$ is the sum of the travel costs of the arc set, $A(R)$, traversed by route $R$.

The VRPTW consists of designing at most $m$ routes of minimum total cost such that each customer is visited exactly once by exactly one route.

The following notation is used in the rest of this chapter. Given a set of vertices $S \subseteq V$, we indicate with $q(S)=\sum_{i \in S} q_{i}$ the total demand of the customers of the set $S$ and with $k(S)$ the minimum number of vehicles needed to serve all customers of the set $S$. We also denote with $q_{\text {min }}=\min \left\{\min _{i \in V}\left\{q_{i}\right\}, q(V)-(m-1) Q\right\}$ the minimum customer demand delivered by any feasible route. Finally, we denote with $z_{U B}$ a valid upper bound on the optimal solution cost of the VRPTW.

We also define a forward path $F=\left(0, i_{1}, \ldots, i_{k-1}, i_{k}\right)$ as an elementary path that starts from depot 0 at time $e_{0}$, visits vertices $V(F)=\left\{0, i_{1}, \ldots, i_{k-1}, i_{k}\right\}$ within their time windows, and ends at customer $\sigma_{F}=i_{k}$ at time $t_{F} \in\left[e_{\sigma_{F}}, l_{\sigma_{F}}\right]$. We denote by $A(F)$ the set of arcs traversed by path $F$ and by $c(F)=\sum_{(i, j) \in A(F)} d_{i j}$ the cost of path $F$. Similarly, a backward path $B=\left(\sigma_{B}=i_{k}, i_{k+1}, \ldots, i_{h}, 0\right)$ is a path that starts from vertex $\sigma_{B}$ at time $t_{B}$, visits customers $V(B)=\left\{i_{k}, i_{k+1}, \ldots, i_{h}, 0\right\}$ within their time windows, and ends at the depot 0 before time $l_{0}$. 


\subsubsection{Set Partitioning Formulation}

Let $\mathscr{R}$ be the index set of all feasible routes in graph $G$, and let $a_{i \ell}, i \in V^{\prime}, \ell \in \mathscr{R}$, be a binary coefficient equal to 1 if $i \in V\left(R_{\ell}\right), 0$ otherwise (we assume that $a_{0 \ell}=1$, for each route $\ell \in \mathscr{R})$. Each route $\ell \in \mathscr{R}$ has an associated cost $c_{\ell}$. Let $x_{\ell}, \ell \in \mathscr{R}$, be a binary variable equal to 1 if and only if route $\ell$ is in the optimal solution ( 0 otherwise). The SP formulation of the VRPTW is

$$
\begin{aligned}
(P) \quad z(P)=\min & \sum_{\ell \in \mathscr{R}} c_{\ell} x_{\ell}, \\
\text { s.t. } & \sum_{\ell \in \mathscr{R}} a_{i \ell} x_{\ell}=1, \quad i \in V, \\
& \sum_{\ell \in \mathscr{R}} x_{\ell} \leq m, \\
& x_{\ell} \in\{0,1\}, \quad \ell \in \mathscr{R} .
\end{aligned}
$$

Constraints (4.2) specify that each customer $i \in V$ must be visited by exactly one route. Constraint (4.3) requires that at most $m$ routes are selected.

\subsubsection{Relaxation $L P$}

The linear relaxation of formulation $P$ can be strengthened with the following valid inequalities.

i) Capacity constraints (CCs). Let $\mathscr{S}=\{S: S \subseteq V,|S| \geq 2\}$ be the set of all subsets of vertices of cardinality greater than 1 , and let $\rho_{\ell}(S)=\mid\left\{(i, j) \in A\left(R_{\ell}\right)\right.$ : $\left.i \in V^{\prime} \backslash S, j \in S\right\} \mid$ be the number of times route $\ell \in \mathscr{R}$ enters the set of vertices $S$. The capacity constraints $(\mathrm{CCs})$ are

$$
\sum_{\ell \in \mathscr{R}} \rho_{\ell}(S) x_{\ell} \geq k(S), \quad S \in \mathscr{S}
$$

In solving the VRPTW, we ignore CCs, whereas in solving the CVRP we consider only a subset of CCs a priori generated as described in Baldacci et al. [2008].

ii) Strengthened capacity constraints (SCs). These inequalities, introduced by Baldacci et al. [2004], lift CCs and are given by inequalities (4.5) where the route coefficient $\rho_{\ell}(S)$ is equal to 1 if $V\left(R_{\ell}\right) \cap S \neq \varnothing$ and 0 otherwise.

iii) Subset-row inequalities (SR3s). Let $\mathscr{C}=\{C \subseteq V:|C|=3\}$ be the set of all customer triplets, and let $\mathscr{R}(C) \subseteq \mathscr{R}$ be the subset of routes serving at least two customers in $C$ (i.e., $\mathscr{R}(C)=\left\{\ell \in \mathscr{R}:\left|V\left(R_{\ell}\right) \cap C\right| \geq 2\right\}$ ). Subset-row inequalities (SR3s) are

$$
\sum_{\ell \in \mathscr{R}(C)} x_{\ell} \leq 1, \quad C \in \mathscr{C}
$$


Inequalities SR3s correspond to a subset of SRs and clique inequalities used by Jepsen et al. [2008] for solving the VRPTW and by Baldacci et al. [2008] for solving the CVRP, respectively. Hereafter, with $C \in \mathscr{C}$ we refer to both the index and the customer triplet of an SR3 inequality.

iv) Weak subset-row inequalities (WSR3s). These inequalities are a relaxed form of the SR3s (4.6) where, given the triplet $C \in \mathscr{C}$, the route set $\mathscr{R}(C)$ contains only those routes traversing at least one $\operatorname{arc}(i, j)$ with $i, j \in C$. The reason for using WSR3s instead of SR3s is that WSR3 dual variables can be implicitly considered in solving the pricing problem. The SR3s (4.6) and, thus, the WSR3s are separated by complete enumeration.

We denote by $L P$ the linear relaxation of formulation $P$ strengthened with inequalities (4.5) and (4.6) and by $z(L P)$ its optimal solution cost. Moreover, we denote by $D$ the dual of problem $L P$. The dual variables are given by the vectors $\boldsymbol{u}=\left(u_{0}, u_{1}, \ldots, u_{n}\right)$, $\boldsymbol{v}=\left(v_{1}, v_{2}, \ldots, v_{|\mathscr{S}|}\right)$, and $\boldsymbol{g}=\left(g_{1}, g_{2}, \ldots, g_{|\mathscr{C}|}\right)$, where $u_{1}, \ldots, u_{n} \in \mathbb{R}$ are the dual variables of constraints (4.2), $u_{0} \in \mathbb{R}_{-}$is the dual variable associated with constraint (4.3), $\boldsymbol{v} \in \mathbb{R}_{+}^{|\mathscr{S}|}$ are the dual variables of inequalities (4.5), and $\boldsymbol{g} \in \mathbb{R}_{-}^{|\mathscr{C}|}$ are the dual variables of inequalities (4.6).

By enlarging the route set $\mathscr{R}$ to contain also nonnecessarily elementary routes, we can design efficient dual-ascent heuristic procedures to find near-optimal solutions of $D$. In $\S 4.5$, we describe three bounding procedures, called $H^{1}, H^{2}$ and $H^{3}$, where procedure $H^{k}$ provides lower bound $L B_{k}$ corresponding to the cost of both a feasible $D$ solution $\left(\boldsymbol{u}^{k}, \boldsymbol{v}^{\boldsymbol{k}}, \boldsymbol{g}^{\boldsymbol{k}}\right)$ and a nonnecessarily feasible $P$ solution $\boldsymbol{x}^{\boldsymbol{k}}$. In $\S 4$.6, we describe a column-and-cut generation bounding procedure, called $H^{4}$, for solving problem $L P$, that computes lower bound $L B_{4}$ corresponding to the $D$ solution $\left(\boldsymbol{u}^{\mathbf{4}}, \boldsymbol{v}^{\mathbf{4}}, \boldsymbol{g}^{\mathbf{4}}\right)$. In the following, we denote with $c_{\ell}^{k}$ the reduced cost of route $\ell \in \mathscr{R}$ with respect to the $D$ solution $\left(\boldsymbol{u}^{k}, \boldsymbol{v}^{k}, \boldsymbol{g}^{\boldsymbol{k}}\right)$.

Procedure $H^{4}$ differs from classical column-and-cut generation methods for the new strategy for solving the pricing problem and the use of the $D$ solution $\left(\boldsymbol{u}^{\mathbf{3}}, \boldsymbol{v}^{\mathbf{3}}, \boldsymbol{g}^{\mathbf{3}}\right)$ to reduce the size of the route set $\mathscr{R}$ by removing any route such that $c_{\ell}^{3}>z_{U B}-L B_{3}$. The usage of the $D$ solution $\left(\boldsymbol{u}^{\mathbf{3}}, \boldsymbol{v}^{\mathbf{3}}, \boldsymbol{g}^{\mathbf{3}}\right)$ has the main benefits of (i) eliminating routes of negative reduced cost when solving the pricing problem in $H^{4}$ and (ii) improving the final lower bound $L B_{4}$. Procedures $H^{1}, H^{2}, H^{3}$ and $H^{4}$ are executed in sequence, and the solution $\left(\boldsymbol{u}^{\boldsymbol{k}}, \boldsymbol{v}^{\boldsymbol{k}}, \boldsymbol{g}^{\boldsymbol{k}}\right)$ of $H^{k}$ is used to hot-start procedure $H^{k+1}, k=1,2,3$.

\subsection{3 $n g$-Route Relaxation for the CVRP}

In $§ 3.4 .2$, we introduced the $n g$-route relaxation for the TSPTW. The same route relaxation can be used for obtaining valid lower bounds to the VRPTW, as well. In particular, it is used in bounding procedures $H^{2}, H^{3}$, and $H^{4}$ for solving the pricing 
subproblems. In this chapter, as route relaxations of VRPTW elementary routes, we use $(t, i)$-routes and $(N G, t, i)$-routes, which correspond exactly to $t$-tours and $n g$-tours described in $\S \S 3.4 .1$ and 3.4.2. As to the CVRP, we use the $(q, i)$-path and the $n g$-route relaxations that can be described as follows.

A $(q, i)$-path is a nonnecessarily elementary path that starts from the depot, visits a set of customers of total demand equal to $q$, and ends at vertex $i \in V^{\prime}$. The cost $f(q, i)$ of a least-cost $(q, i)$-path can be computed as described by Christofides et al. [1981b]. A $(q, i)$-route is a $(q, 0)$-path.

A forward $n g$-path $(N G, q, i)$ is a nonnecessarily elementary path $F=\left(0, i_{1}, \ldots, i_{k-1}\right.$, $i_{k}=i$ ) that starts from the depot 0 , visits a set of customers (each once or more) of total demand equal to $q$ such that $N G=\Pi_{F}$, ends at customer $i \in V^{\prime}$, and such that $i \notin \Pi_{F^{\prime}}$, where $F^{\prime}=\left(0, i_{1}, \ldots, i_{k-1}\right)$.

We denote by $f(N G, q, i)$ the cost of a least-cost forward $n g$-path $(N G, q, i)$. An $(N G, q, i)$-route is an $(N G, q, 0)$-path. Functions $f(N G, q, i)$ can be computed using DP recursions similar to (3.3) as follows.

Define the state-space graph $\widehat{\widehat{G}_{F}}=\left(\widehat{\mathscr{F}}, \widehat{\mathscr{A}_{F}}\right)$, where the vertex set is defined as

$$
\widehat{\mathscr{F}}=\left\{(N G, q, i): i \in V^{\prime}, q \in\left[q_{i}, Q\right], \forall N G \subseteq N_{i} \text { s.t. } N G \ni i \text { and } \sum_{j \in N G} q_{j} \leq q\right\},
$$

and the arc set as

$$
\begin{gathered}
\widehat{\mathscr{A}_{F}}=\left\{\left(\left(N G^{\prime}, q^{\prime}, j\right),(N G, q, i)\right):\left(N G^{\prime}, q^{\prime}, j\right),(N G, q, i) \in \widehat{\mathscr{F}}\right. \\
\left.j \in \Gamma_{i}^{-1}, q^{\prime}=q-q_{i}, \forall N G^{\prime} \subseteq N_{j} \text { s.t. } N G^{\prime} \ni j \text { and } N G^{\prime} \cap N_{i}=N G \backslash\{i\}\right\} .
\end{gathered}
$$

The DP recursion for computing functions $f(N G, q, i)$ is

$$
f(N G, q, i)=\min _{\left(N G^{\prime}, q^{\prime}, j\right):\left(\left(N G^{\prime}, q^{\prime}, j\right),(N G, q, i)\right) \in \widehat{\mathscr{A}}_{F}}\left\{f\left(N G^{\prime}, q^{\prime}, j\right)+d_{j i}\right\}, \quad(N G, q, i) \in \widehat{\mathscr{F}} .
$$

By using the transpose of the cost matrix $\left[d_{i j}\right]$, we can compute the reverse functions $f^{-1}(q, i)$ and $f^{-1}(N G, q, i)$ with recursions similar to those used for $f(q, i)$ and $f(N G, q, i)$. For symmetric CVRPs, we have $f^{-1}(q, i)=f(q, i)$ and $f^{-1}(N G, q, i)=$ $f(N G, q, i)$.

\subsection{An Exact Algorithm}

In this section, we describe an exact algorithm for solving both the VRPTW and the CVRP. The algorithm generates a reduced problem $\widetilde{P}$ obtained from $P$ by replacing the route set $\mathscr{R}$ with a smaller route set $\widetilde{\mathscr{R}} \subseteq \mathscr{R}$ containing any optimal solution and 
solves $\widetilde{P}$ with an IP solver. The core of the algorithm is the bounding procedures $H^{1}$, $H^{2}, H^{3}$, and $H^{4}$ described in $\S \S 4.5$ and 4.6. The algorithm can be described as follows.

Step 1. Execute in sequence bounding procedures $H^{1}, H^{2}$ and $H^{3}$. If the $L P$ solution $\boldsymbol{x}^{k}$ corresponding to lower bound $L B_{k}$ is a feasible $P$ solution, for some $k \in$ $\{1,2,3\}, \boldsymbol{x}^{k}$ is an optimal solution, stop.

Step 2. Call procedure GenR (see $\S 4.5 .5$ ) to generate the largest route set $\mathscr{R}^{3} \subseteq \mathscr{R}$ such that (i) $c_{\ell}^{3} \leq z_{U B}-L B_{3}, \ell \in \mathscr{R}^{3}$, and (ii) $\left|\mathscr{R}^{3}\right| \leq \Delta(\mathscr{R})$, where $\Delta(\mathscr{R})$ is a parameter. If $\left|\mathscr{R}^{3}\right|<\Delta(\mathscr{R}), \mathscr{R}^{3}$ contains the routes of any optimal solution of cost less than or equal to $z_{U B}$ and is defined optimal. Otherwise, $\mathscr{R}^{3}$ is defined nonoptimal.

Step 3. Call procedure $H^{4}$ to compute lower bound $L B_{4}$ corresponding to the cost of the $D$ solution $\left(\boldsymbol{u}^{\mathbf{4}}, \boldsymbol{v}^{\mathbf{4}}, \boldsymbol{g}^{\mathbf{4}}\right)$. If the optimal $L P$ solution $\boldsymbol{x}^{\mathbf{4}}$ is integer, stop.

Step 4. We have two cases

(a) If the route set $\mathscr{R}^{3}$ is optimal, select the subset $\widetilde{R} \subseteq \mathscr{R}^{3}$ of routes such that $c_{\ell}^{4} \leq z_{U B}-L B_{4}$.

(b) If the route set $\mathscr{R}^{3}$ is nonoptimal, call GenRF (see $\S 4.7$ ) to compute the set of routes $\widetilde{\mathscr{R}}$ such that

$$
\left.\begin{array}{ll}
c_{\ell}^{3} \leq z_{U B}-L B_{3} & \ell \in \widetilde{\mathscr{R}} \\
c_{\ell}^{4} \leq z_{U B}-L B_{4} & \ell \in \mathscr{\mathscr { R }} \\
|\widetilde{\mathscr{R}}| \leq \Delta(\mathscr{R}) &
\end{array}\right\}
$$

If $|\widetilde{\mathscr{R}}|<\Delta(\mathscr{R})$, then $\widetilde{\mathscr{R}}$ contains the routes of any optimal solution and is defined optimal.

Step 5. Solve problem $\widetilde{P}$ derived from $P$ by replacing the route set $\mathscr{R}$ with the route set $\widetilde{\mathscr{R}}$ and by adding the subsets of CCs and SR3s saturated by the optimal $L P$ solution produced by $H^{4}$. Let $z(\widetilde{P})$ be the cost of the optimal solution $\tilde{\boldsymbol{x}}$ of $\widetilde{P}$ (we assume $z(\widetilde{P})=\infty$ if no feasible solution exists). If the route set $\widetilde{R}$ is optimal, then $\tilde{\boldsymbol{x}}$ is an optimal solution; otherwise, $\min \left\{z(\widetilde{P}), z_{U B}\right\}$ is a valid upper bound to $z(P)$.

\subsection{Bounding Procedures $H^{1}, H^{2}$ and $H^{3}$}

Bounding procedures $H^{1}, H^{2}$ and $H^{3}$ use the same column-and-cut generation method, called CCG, to find lower bounds $L B_{1}, L B_{2}$ and $L B_{3}$ corresponding to the cost of three near-optimal $D$ solutions. Procedures $H^{1}$ and $H^{2}$ are based on two different route relaxations and add CCs. Procedure $H^{3}$ is based on elementary routes and adds both SCs and WSR3s. 


\subsubsection{Algorithm CCG}

Algorithm CCG differs from standard column-and-cut generation methods based on the simplex algorithm because it uses a dual-ascent heuristic algorithm to solve the master problem. Procedure $C C G$ was proposed by Baldacci et al. [2008] for the CVRP and used by Boschetti et al. [2008] for the set partitioning problem, who showed that $C C G$ is faster than the simplex because it is not affected by the typical degeneracy of the simplex.

Define $A(C)=\{(i, j) \in A: i, j \in C\}$, and let $\mathscr{C}_{\ell}=\left\{C \in \mathscr{C}:\left|A(C) \cap A\left(R_{\ell}\right)\right| \geq 1\right\}$ be the subset of WSR3s involving the route $\ell$. Algorithm $C C G$ is based on the following theorem, which is a straightforward extension of Theorem 1 described in §3.5.2.

Theorem 1. Let $\boldsymbol{\lambda}=\left(\lambda_{0}, \lambda_{1}, \ldots, \lambda_{n}\right), \boldsymbol{\mu}=\left(\mu_{1}, \ldots, \mu_{|\mathscr{S}|}\right), \boldsymbol{\omega}=\left(\omega_{1}, \ldots, \omega_{|\mathscr{C}|}\right)$ be three

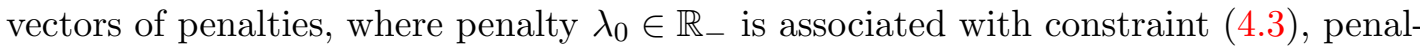
ties $\lambda_{i} \in \mathbb{R}, i \in V$, with constraints (4.2), penalties $\mu_{S} \in \mathbb{R}_{+}, S \in \mathscr{S}$, with constraints (4.5) in the form of either SCs or CCs, and penalties $\omega_{C} \in \mathbb{R}_{-}, C \in \mathscr{C}$, with constraints (4.6) in the relaxed form of WSR3s. Let $\widehat{\mathscr{R}}$ be the index set of nonnecessarily elementary routes given by some SSR. A feasible dual solution $(\boldsymbol{u}, \boldsymbol{v}, \boldsymbol{g})$ of problem $L P$ of cost $z(D(\boldsymbol{\lambda}, \boldsymbol{\mu}, \boldsymbol{\omega}))$ is obtained as

$$
\left.\begin{array}{ll}
u_{i}=b_{i}+\lambda_{i} & i \in V \\
u_{0}=\lambda_{0} & \\
v_{S}=\mu_{S} & S \in \mathscr{S} \\
g_{C}=\omega_{C} & C \in \mathscr{C}
\end{array}\right\}
$$

where $b_{i}, i \in V$, is defined as

$$
b_{i}=q_{i} \min _{\ell \in \widehat{\mathscr{R}}: a_{i \ell} \geq 1}\left\{\frac{c_{\ell}-\sum_{i \in V^{\prime}} a_{i \ell} \lambda_{i}-\sum_{S \in \mathscr{S}} \rho_{\ell}(S) \mu_{S}-\sum_{C \in \mathscr{C}_{\ell}} \omega_{C}}{\sum_{i \in V} a_{i \ell} q_{i}}\right\} .
$$

Proof. Consider a route $\ell \in \widehat{\mathscr{R}}$. From the definition of the dual solution $(\boldsymbol{u}, \boldsymbol{v}, \boldsymbol{g})$, we derive

$$
u_{i} \leq q_{i} \frac{c_{\ell}-\sum_{i \in V^{\prime}} a_{i \ell} \lambda_{i}-\sum_{S \in \mathscr{S}} \rho_{\ell}(S) \mu_{S}-\sum_{C \in \mathscr{C}_{\ell}} \omega_{C}}{\sum_{i \in V} a_{i \ell} q_{i}}+\lambda_{i}, \quad i \in V: a_{i \ell} \geq 1 .
$$

Thus, for each route $\ell \in \widehat{\mathscr{R}}$, the following relation holds

$$
\sum_{i \in V^{\prime}} a_{i \ell} u_{i} \leq \sum_{i \in V^{\prime}} a_{i \ell} q_{i} \frac{c_{\ell}-\sum_{i \in V^{\prime}} a_{i \ell} \lambda_{i}-\sum_{S \in \mathscr{S}} \rho_{\ell}(S) \mu_{S}-\sum_{C \in \mathscr{C}_{\ell}} \omega_{C}}{\sum_{i \in V} a_{i \ell} q_{i}}+\sum_{i \in V^{\prime}} a_{i \ell} \lambda_{i}
$$




$$
=c_{\ell}-\sum_{S \in \mathscr{S}} \rho_{\ell}(S) \mu_{S}-\sum_{C \in \mathscr{C}_{\ell}} \omega_{C},
$$

which corresponds to the constraint of problem $D$ for route $\ell \in \widehat{\mathscr{R}}$

$$
\sum_{i \in V^{\prime}} a_{i \ell} u_{i}+\sum_{S \in \mathscr{S}} \rho_{\ell}(S) v_{S}+\sum_{C \in \mathscr{b}_{\ell}} g_{C} \leq c_{\ell \cdot \square}
$$

Algorithm $C C G$ is a column-and-cut generation-like method for solving the problem

$$
L C G=\max _{(\boldsymbol{\lambda}, \boldsymbol{\mu}, \boldsymbol{\omega})}\{z(D(\boldsymbol{\lambda}, \boldsymbol{\mu}, \boldsymbol{\omega}))\}
$$

Procedure $C C G$ executes a number of macro-iterations to compute a dual solution $\left(\boldsymbol{u}^{\prime}, \boldsymbol{v}^{\prime}, \boldsymbol{g}^{\prime}\right)$ of the master problem, defined by the route subset $\mathscr{R}^{\prime} \subseteq \mathscr{R}$, solving problem (4.11) with a predefined number Maxit2 of subgradient iterations to modify the penalty vectors $(\boldsymbol{\lambda}, \boldsymbol{\mu}, \boldsymbol{\omega})$.

Baldacci et al. [2008] have shown that a valid subgradient of function $z(D(\boldsymbol{\lambda}, \boldsymbol{\mu}, \boldsymbol{\omega}))$ at point $(\boldsymbol{\lambda}, \boldsymbol{\mu}, \boldsymbol{\omega})$ can be computed by associating, with the current $D$ solution, a nonnecessarily feasible $L P$ solution $\boldsymbol{x}$ of cost $z(L P)=z(D(\boldsymbol{\lambda}, \boldsymbol{\mu}, \boldsymbol{\omega}))$ defined as follows. Let $\widetilde{\mathscr{R}}$ be the index set of the distinct routes producing $b_{i}, i \in V$, in expressions (4.10), and let $\ell(i)$ be the index of the route in $\widetilde{R}$ associated with $b_{i}, i \in V$. Solution $\boldsymbol{x}$ is computed as $x_{\ell}=\sum_{i \in V} \frac{a_{i \ell} q_{i}}{\sum_{i \in V} a_{i \ell} q_{i}} \xi_{\ell}^{i}, \ell \in \widetilde{\mathscr{R}}$, by setting $\xi_{\ell(i)}^{i}=1$ and $\xi_{\ell}^{i}=0$, for each route $\ell \in \widetilde{\mathscr{R}} \backslash\{\ell(i)\}$, and each customer $i \in V$. The values of the left-hand-side of constraints (4.2), (4.3), (4.5), and (4.6) with respect to $\boldsymbol{x}$ are used to update penalty vectors $\boldsymbol{\lambda}, \boldsymbol{\mu}$, and $\boldsymbol{\omega}$ by means of the usual subgradient expressions, where we use $z_{U B}=1.2 z(L P)$ instead of a feasible upper bound.

The set $\mathscr{S}$ of CCs (or SCs) is generated a priori (see $\S 4.3$ ). After computing the master dual solution $\left(\boldsymbol{u}^{\prime}, \boldsymbol{v}^{\prime}, \boldsymbol{g}^{\prime}\right), C C G$ adds to the master a subset of WSR3 inequalities violated by the $L P$ solution $\boldsymbol{x}$. Moreover, $C C G$ generates a subset $\mathscr{N}$ of routes of negative reduced cost with respect to $\left(\boldsymbol{u}^{\prime}, \boldsymbol{v}^{\prime}, \boldsymbol{g}^{\prime}\right)$. If $\mathscr{N} \neq \varnothing$, then $C C G$ sets $\mathscr{R}^{\prime}=\mathscr{R}^{\prime} \cup \mathscr{N}$; otherwise, $\left(\boldsymbol{u}^{\prime}, \boldsymbol{v}^{\prime}, \boldsymbol{g}^{\prime}\right)$ is a feasible $D$ solution. $C C G$ terminates after Maxit1 macro iterations (Maxit1 defined a priori).

We denote by $\left(\boldsymbol{u}^{*}, \boldsymbol{v}^{*}, \boldsymbol{g}^{*}\right)$ and $\boldsymbol{x}^{*}$ the final $D$ and $L P$ solutions of cost $L C G$ achieved by $C C G$ using penalty vectors $\left(\lambda^{*}, \boldsymbol{\mu}^{*}, \boldsymbol{\omega}^{*}\right)$, respectively.

A step-by-step description of procedure $C C G$ is the following.

Step 1. Initialization. A route set $\mathscr{R}^{\prime} \subseteq \widehat{\mathscr{R}}$ is generated to initialize the master problem which corresponds to $L P$, where $\mathscr{R}$ is replaced with $\mathscr{R}^{\prime}$. We assume that $\mathscr{R}^{\prime}$ contains at least one route passing through each customer $i \in V$. The route set $\mathscr{R}^{\prime}$ and the subsets $\mathscr{S}$ and $\mathscr{C}$ of SCs and WSR3s are generated, and $(\boldsymbol{\lambda}, \boldsymbol{\mu}, \boldsymbol{\omega})$ 
are initialized as described in $\S \S 4.5 .2,4.5 .3$, and 4.5.4. Set $L C G=0$ and iter $=1$.

Step 2. Find a master dual solution $\left(\boldsymbol{u}^{\prime}, \boldsymbol{v}^{\prime}, \boldsymbol{g}^{\prime}\right)$ of cost $z^{\prime}$. A near-optimal dual solution $\left(\boldsymbol{u}^{\prime}, \boldsymbol{v}^{\prime}, \boldsymbol{g}^{\prime}\right)$ of cost $z^{\prime}$ of the master problem is computed by an iterative procedure that initializes $z^{\prime}=0$ and performs Maxit2 iterations of the following operations:

(i) Compute a dual solution $(\boldsymbol{u}, \boldsymbol{v}, \boldsymbol{g})$ of the master of cost $z$ by means of expressions (4.9) and (4.10), where $\widehat{\mathscr{R}}$ is replaced with $\mathscr{R}^{\prime}$, using the current vectors $(\boldsymbol{\lambda}, \boldsymbol{\mu}, \boldsymbol{\omega})$. Let $\widetilde{\mathscr{R}}$ be the index set of the distinct routes producing $b_{i}, i \in V$, in expressions (4.10), and let $\ell(i)$ be the index of the route in $\mathscr{R}^{\prime}$ associated with $b_{i}, i \in V$. Define a nonnecessarily feasible $L P$ solution $\boldsymbol{x}$ as $x_{\ell}=\sum_{i \in V} \frac{a_{i \ell} q_{i}}{\sum_{i \in V} a_{i \ell} q_{i}} \xi_{\ell}^{i}, \ell \in \widetilde{\mathscr{R}}$, by setting $\xi_{\ell(i)}^{i}=1$, and $\xi_{\ell}^{i}=0$, for each route $\ell \in \widetilde{\mathscr{R}} \backslash\{\ell(i)\}$, and each customer $i \in V$. If $z>z^{\prime}$, update $z^{\prime}=z, \boldsymbol{x}^{\prime}=\boldsymbol{x},\left(\boldsymbol{u}^{\prime}, \boldsymbol{v}^{\prime}, \boldsymbol{g}^{\prime}\right)=(\boldsymbol{u}, \boldsymbol{v}, \boldsymbol{g})$.

(ii) Update the penalty vectors $(\boldsymbol{\lambda}, \boldsymbol{\mu}, \boldsymbol{\omega})$ as follows. Compute

- $\alpha_{0}=\sum_{\ell \in \widetilde{\mathscr{R}}} x_{\ell}$ and $\alpha_{i}=\sum_{\ell \in \widetilde{\mathscr{R}}} a_{i \ell} x_{\ell}, i \in V$;

- $\beta_{S}=\sum_{\ell \in \widetilde{\mathscr{R}}} b_{\ell}(S) x_{\ell}, S \in \mathscr{S}$

- $\delta_{C}=\sum_{\ell \in \mathscr{R}(C) \cap \widetilde{\mathscr{R}}}\left\lfloor\frac{\sum_{i \in C} a_{i \ell}}{2}\right\rfloor x_{\ell}, C \in \mathscr{C}$.

Then, vectors $(\boldsymbol{\lambda}, \boldsymbol{\mu}, \boldsymbol{\omega})$ are modified as follows

$$
\left.\begin{array}{ll}
\lambda_{i}=\lambda_{i}-\epsilon \gamma\left(\alpha_{i}-1\right), & i \in V \\
\lambda_{0}=\min \left\{0, \lambda_{0}-\epsilon \gamma\left(\alpha_{0}-m\right)\right\} & \\
\mu_{S}=\max \left\{0, \mu_{S}-\epsilon \gamma\left(\beta_{S}-k(S)\right\},\right. & S \in \mathscr{S} \\
\omega_{C}=\min \left\{0, \omega_{C}-\epsilon \gamma\left(\delta_{C}-1\right)\right\}, & C \in \mathscr{C}
\end{array}\right\}
$$

where $\epsilon$ is a positive constant and

$$
\gamma=\frac{0.2 z^{\prime}}{\sum_{i \in V}\left(\alpha_{i}-1\right)^{2}+\left(\alpha_{0}-m\right)^{2}+\sum_{S \in \mathscr{S}}(\beta(S)-k(S))^{2}+\sum_{C \in \mathscr{C}}\left(\delta_{C}-1\right)^{2}} .
$$

Step 3. Check if $\left(\boldsymbol{u}^{\prime}, \boldsymbol{v}^{\prime}, \boldsymbol{g}^{\prime}\right)$ is a feasible D solution. Generate (see $\S \S 4.5 .2,4.5 .3$, and 4.5.4) the largest subset $\mathscr{N} \subseteq \widehat{\mathscr{R}}$ of routes of negative reduced cost with respect to the current dual master solution $\left(\boldsymbol{u}^{\prime}, \boldsymbol{v}^{\prime}, \boldsymbol{g}^{\prime}\right)$ and such that $|\mathscr{N}| \leq \Delta(\mathscr{N})$ $\left(\Delta(\mathscr{N})\right.$ is an a priori defined parameter). If $\mathscr{N}=\varnothing$ and $z^{\prime}$ is greater than $L C G$, then $L C G=z^{\prime},\left(\boldsymbol{u}^{*}, \boldsymbol{v}^{*}, \boldsymbol{g}^{*}\right)=\left(\boldsymbol{u}^{\prime}, \boldsymbol{v}^{\prime}, \boldsymbol{g}^{\prime}\right), \boldsymbol{x}^{*}=\boldsymbol{x}^{\prime}$ and $\left(\boldsymbol{\lambda}^{*}, \boldsymbol{\mu}^{*}, \boldsymbol{\omega}^{*}\right)=$ $(\boldsymbol{\lambda}, \boldsymbol{\mu}, \boldsymbol{\omega})$; otherwise, $\mathscr{R}^{\prime}=\mathscr{R}^{\prime} \cup \mathscr{N}$ is updated.

Step 4. Termination criterion. Set iter $=i t e r+1$. If $i t e r=$ Maxit 1 , stop. 
Step 5. Separate WSR3s. Find the set $\mathscr{C}^{\prime}$ of the WSR3s most violated by the current $L P$ solution $\boldsymbol{x}^{\prime}$ obtained at Step 2 and such that $\left|\mathscr{C}^{\prime}\right| \leq \Delta(\mathscr{C})$. WSR3s are separated by complete enumeration.

\subsubsection{Bounding Procedure $H^{1}$}

Bounding procedure $H^{1}$ enlarges the route set $\mathscr{R}$ with the $t$-routes (to solve the VRPTW) or the $q$-routes (in the CVRP, see Christofides et al. [1981b]). The initial route set $\mathscr{R}^{\prime}$ of the master problem contains all single-customer routes $(0, i, 0)$, $i \in V$. WSR3s are ignored (i.e., we set $\mathscr{C}=\varnothing$ ); solving the VRPTW, we initialize $\mathscr{S}=\varnothing$, whereas in the CVRP the set $\mathscr{S}$ contains CCs and is generated a priori as described in Baldacci et al. [2008]. We initialize $(\boldsymbol{\lambda}, \boldsymbol{\mu}, \boldsymbol{\omega})=(\mathbf{0}, \mathbf{0}, \mathbf{0})$.

Define the modified arc cost $d_{i j}^{\prime}=d_{i j}-\frac{1}{2}\left(u_{i}^{\prime}+u_{j}^{\prime}\right)-\sum_{S \in \mathscr{S}_{i j}} v_{S}^{\prime},(i, j) \in A$, with respect to the current dual solution $\left(\boldsymbol{u}^{\prime}, \boldsymbol{v}^{\prime}, \boldsymbol{g}^{\prime}\right)$, where $\mathscr{S}_{i j}=\left\{S \in \mathscr{S}:(i, j) \in A, i \in V^{\prime} \backslash S, j \in\right.$ $S\}$. The set $\mathscr{N}$ is computed as follows. If we use $t$-routes, we compute functions $f(t, i)$ using the modified arc costs $d_{i j}^{\prime}$ instead of $d_{i j}$. Let $h(i)=\min _{t \in\left[e_{i}, l_{i}\right]}\left\{f(t, i)+d_{i 0}^{\prime}\right\}$. The set $\mathscr{N}$ contains any $t$-route corresponding to $h(i)<0, i \in V$. Similarly, we compute $\mathscr{N}$ when $\widehat{\mathscr{R}}$ contains $q$-routes.

At the end, $H^{1}$ sets $\left(\boldsymbol{u}^{\mathbf{1}}, \boldsymbol{v}^{\mathbf{1}}, \boldsymbol{g}^{\mathbf{1}}\right)=\left(\boldsymbol{u}^{*}, \boldsymbol{v}^{*}, \boldsymbol{g}^{*}\right), \boldsymbol{x}^{\mathbf{1}}=\boldsymbol{x}^{*},\left(\lambda^{\mathbf{1}}, \boldsymbol{\mu}^{\mathbf{1}}, \boldsymbol{\omega}^{\mathbf{1}}\right)=\left(\lambda^{*}, \boldsymbol{\mu}^{*}, \omega^{*}\right)$, $L B_{1}=L C G$.

\subsubsection{Bounding Procedure $H^{2}$}

Procedure $H^{2}$ enlarges the route set $\mathscr{R}$ with the $n g$-routes and adds to $L P$ the same set $\mathscr{S}$ of CCs used by $H^{1}$. WSR3s (4.6) are ignored. We initialize $(\boldsymbol{\lambda}, \boldsymbol{\mu}, \boldsymbol{\omega})=\left(\boldsymbol{\lambda}^{\mathbf{1}}, \boldsymbol{\mu}^{\mathbf{1}}, \boldsymbol{\omega}^{\mathbf{1}}\right)$, define $d_{i j}^{1}=d_{i j}-\frac{1}{2}\left(u_{i}^{1}+u_{j}^{1}\right)-\sum_{S \in \mathscr{S}_{i j}} v_{S}^{1},(i, j) \in A$, and compute $N_{i}$ to be the $\Delta\left(N_{i}\right)$ nearest customers to $i$ according to $d_{i j}^{1}$. We compute functions $f(N G, t, i)$ using $d_{i j}^{1}$ instead of $d_{i j}$ in recursions (4.7) and the costs $h(i)=\min _{(N G, t, i) \in \widehat{\mathscr{F}}}\left\{f(N G, t, i)+d_{i 0}^{1}\right\}$, $i \in V$, of the least-cost $n g$-route visiting $i$ immediately before arriving at the depot. The route set $\mathscr{R}^{\prime}$ contains the $n g$-routes corresponding to $h(i)<0, i \in V$.

At each iteration, to generate the set $\mathscr{N}$, we compute functions $f(N G, t, i)$ with the modified arc cost $d_{i j}^{\prime},(i, j) \in A$, and $N_{i}$ contains the $\Delta\left(N_{i}\right)$ nearest customers to $i$ according to $d_{i j}^{\prime}$. The route set $\mathscr{N}$ contains every $n g$-route corresponding to $h(i)<0$, $i \in V$.

At the end, procedure $H^{2}$ sets $\left(\boldsymbol{u}^{\mathbf{2}}, \boldsymbol{v}^{\mathbf{2}}, \boldsymbol{g}^{\mathbf{2}}\right)=\left(\boldsymbol{u}^{*}, \boldsymbol{v}^{*}, \boldsymbol{g}^{*}\right), \boldsymbol{x}^{\mathbf{2}}=\boldsymbol{x}^{*},\left(\boldsymbol{\lambda}^{\mathbf{2}}, \boldsymbol{\mu}^{\mathbf{2}}, \boldsymbol{\omega}^{\mathbf{2}}\right)=$ $\left(\boldsymbol{\lambda}^{*}, \boldsymbol{\mu}^{*}, \boldsymbol{\omega}^{*}\right)$, and $L B_{2}=L C G$. 


\subsubsection{Bounding Procedure $H^{3}$}

Procedure $H^{3}$ uses the set of elementary routes $\mathscr{R}$. The sets $\mathscr{R}^{\prime}$ and $\mathscr{N}$ are generated using the procedure GenR described in $\S 4.5 .5$. Given a $D$ solution $(\tilde{\boldsymbol{u}}, \tilde{\boldsymbol{v}}, \tilde{\boldsymbol{g}})$ and two parameters $\Delta$ and $\gamma$, GenR generates at most $\Delta$ routes of reduced cost less than or equal to $\gamma$ with respect to $(\tilde{\boldsymbol{u}}, \tilde{\boldsymbol{v}}, \tilde{\boldsymbol{g}})$.

The initial route set $\mathscr{R}^{\prime}$ is obtained by setting $\Delta=\Delta^{a},(\tilde{\boldsymbol{u}}, \tilde{\boldsymbol{v}}, \tilde{\boldsymbol{g}})=\left(\boldsymbol{u}^{\mathbf{2}}, \boldsymbol{v}^{\mathbf{2}}, \boldsymbol{g}^{\mathbf{2}}\right)$, and $\gamma=z_{U B}-L B_{2}$, and adding all single-customer routes to $\mathscr{R}^{\prime}$. Moreover, we initialize $(\boldsymbol{\lambda}, \boldsymbol{\mu}, \boldsymbol{\omega})=\left(\boldsymbol{\lambda}^{\mathbf{2}}, \boldsymbol{\mu}^{\mathbf{2}}, \boldsymbol{\omega}^{\mathbf{2}}\right)$. In generating the route set $\mathscr{N}$, we set $\Delta=\Delta^{b}$, $(\tilde{\boldsymbol{u}}, \tilde{\boldsymbol{v}}, \tilde{\boldsymbol{g}})=\left(\boldsymbol{u}^{\prime}, \boldsymbol{v}^{\prime}, \boldsymbol{g}^{\prime}\right)$, and $\gamma=-\varepsilon\left(\right.$ say $\left.\varepsilon=10^{-6}\right)$.

Bounding procedure $H^{3}$ adds, to $L P$, the set $\mathscr{S}$ of CCs used by $H^{1}$ and $H^{2}$ in the form of SCs and separates WSR3s.

At the end, procedure $H^{3}$ sets $\left(\boldsymbol{u}^{\mathbf{3}}, \boldsymbol{v}^{\mathbf{3}}, \boldsymbol{g}^{\mathbf{3}}\right)=\left(\boldsymbol{u}^{*}, \boldsymbol{v}^{*}, \boldsymbol{g}^{*}\right), \boldsymbol{x}^{\mathbf{3}}=\boldsymbol{x}^{*},\left(\boldsymbol{\lambda}^{\mathbf{3}}, \boldsymbol{\mu}^{\mathbf{3}}, \boldsymbol{\omega}^{\mathbf{3}}\right)=$ $\left(\boldsymbol{\lambda}^{*}, \boldsymbol{\mu}^{*}, \boldsymbol{\omega}^{*}\right)$, and $L B_{3}=L C G$.

\subsubsection{Procedure GenR}

Procedure GenR is a DP algorithm that generates elementary routes using bounding functions based on the $n g$-path relaxation. GenR is an extension of the algorithm described by Baldacci et al. [2008] for the symmetric CVRP that, in turn, is based on the method proposed by Mingozzi et al. [1994] and adapted by Baldacci et al. [2006] for the asymmetric CVRP on a multigraph. Similar methods have been used by Righini and Salani [2008], Jepsen et al. [2008], and Desaulniers et al. [2008].

In $\S 4.3$, we gave the definition of forward and backward paths. In addition, for a forward path $F=\left(0, i_{1}, \ldots, i_{k-1}, i_{k}\right)$, we refer to $i_{k-1}$ with $\pi_{F}$ and set $q_{F}=\sum_{i \in V(F)} q_{i}$, and, for a backward path $B=\left(i_{k}, i_{k+1}, \ldots, i_{h}, 0\right)$, we refer to $i_{k+1}$ with $\pi_{B}$ and set $q_{B}=\sum_{i \in V(B)} q_{i}$

Let $\tau$ be a time such that $\tau \in\left(e_{0}, l_{0}\right)$ (say, $\tau=\left\lfloor\left(l_{0}-e_{0}\right) / 2\right\rfloor$ ). We define $\mathscr{F}$ as the set of all forward paths such that $\pi_{F}$ is visited at time less than $\tau, F \in \mathscr{F}$, and $\mathscr{B}$ as the set of all backward paths such that $\pi_{B}$ is visited at time greater than $\tau, B \in \mathscr{B}$, plus all backward paths $B=(k, 0), k \in V$. Procedure GenR is based on the observation that all feasible VRPTW routes can be obtained combining any pair of paths $(F, B)$, $F \in \mathscr{F}, B \in \mathscr{B}$, satisfying the following feasibility conditions

$$
\sigma_{F}=\sigma_{B}, \quad V(F) \cap V(B)=\left\{0, \sigma_{F}\right\}, \quad t_{F} \leq t_{B}, \quad q_{F}+q_{B}-q_{\sigma_{F}} \leq Q .
$$

GenR is a two-phase algorithm. Given a $D$ solution $(\tilde{\boldsymbol{u}}, \tilde{\boldsymbol{v}}, \tilde{\boldsymbol{g}})$, it generates at most $\Delta$ routes of reduced cost less than or equal to $\gamma$. In Phase 1 , GenR generates the path 
sets $\mathscr{F}$ and $\mathscr{B}$ using a procedure called GenP; in Phase 2, it derives feasible routes by combining $\mathscr{F}$ and $\mathscr{B}$ using a procedure called Combine. GenR is based on the following lemma.

Lemma 1. Let $\tilde{d}_{i j}=d_{i j}-\frac{1}{2}\left(\tilde{u}_{i}+\tilde{u}_{j}\right)-\sum_{S \in \mathscr{S}_{i j}} \tilde{v}_{s}$ be the arc reduced costs with respect to $(\tilde{\boldsymbol{u}}, \tilde{\boldsymbol{v}}, \tilde{\boldsymbol{g}})$, and let $\tilde{c}(F)=\sum_{(i, j) \in A(F)} \tilde{d}_{i j}, F \in \mathscr{F}$, and $\tilde{c}(B)=\sum_{(i, j) \in A(B)} \tilde{d}_{i j}, B \in \mathscr{B}$. Let $\ell \in \mathscr{R}$ be the route of reduced cost $\tilde{c}_{\ell}=c_{\ell}-\sum_{i \in V\left(R_{\ell}\right)} \tilde{u}_{i}-\sum_{S \in \mathscr{S}} \rho_{\ell}(S) \tilde{v}_{S}-$ $\sum_{C \in \mathscr{C}_{\ell}} \tilde{g}_{C}$, where $\mathscr{S}$ corresponds to SCs, resulting from a given pair of paths $F$ and $B$ satisfying conditions (4.12). The following inequality holds $\tilde{c}(F)+\tilde{c}(B) \leq \tilde{c}_{\ell}$.

Proof. Because paths $F$ and $B$ satisfy conditions (4.12) and route $\ell$ is the combination of $F$ and $B$, we have

$$
\sum_{(i, j) \in A(F)} d_{i j}+\sum_{(i, j) \in A(B)} d_{i j}=c_{\ell}
$$

and

$$
\frac{1}{2} \sum_{(i, j) \in A(F)}\left(\tilde{u}_{i}+\tilde{u}_{j}\right)+\frac{1}{2} \sum_{(i, j) \in A(B)}\left(\tilde{u}_{i}+\tilde{u}_{j}\right)=\sum_{i \in V\left(R_{\ell}\right)} \tilde{u}_{i} .
$$

From the definitions of $\tilde{d}_{i j}, \tilde{c}(F)$ and $\tilde{c}(B)$ and from expressions (4.13) and (4.14), we derive

$$
\tilde{c}(F)+\tilde{c}(B)=c_{\ell}-\sum_{i \in V\left(R_{\ell}\right)} \tilde{u}_{i}-\sum_{(i, j) \in A\left(R_{\ell}\right)} \sum_{S \in \mathscr{S}_{i j}} \tilde{v}_{S} .
$$

Because $b_{\ell}(S) \in\{0,1\}, S \in \mathscr{S}$, as $\mathscr{S}$ corresponds to SCs and $\tilde{v}_{S} \geq 0$, we have

$$
\sum_{(i, j) \in A\left(R_{\ell}\right)} \sum_{S \in \mathscr{I}_{i j}} \tilde{v}_{S} \geq \sum_{S \in \mathscr{S}} b_{\ell}(S) \tilde{v}_{S}
$$

From expressions (4.15) and (4.16), we obtain

$$
\tilde{c}(F)+\tilde{c}(B) \leq c_{\ell}-\sum_{i \in V\left(R_{\ell}\right)} \tilde{u}_{i}-\sum_{S \in \mathscr{S}} b_{\ell}(S) \tilde{v}_{S} .
$$

Because $\tilde{g}_{C} \leq 0, C \in \mathscr{C}$, from the definition of $\tilde{c}_{\ell}$ and expression (4.17), we derive the lemma. $\square$

GenR is called (i) at the beginning of $H^{3}$ to generate the route set $\mathscr{R}^{\prime}$ of the initial master problem setting $(\tilde{\boldsymbol{u}}, \tilde{\boldsymbol{v}}, \tilde{\boldsymbol{g}})=\left(\boldsymbol{u}^{\mathbf{2}}, \boldsymbol{v}^{\mathbf{2}}, \boldsymbol{g}^{\mathbf{2}}\right)$ and $\gamma=z_{U B}-L B_{2}$; (ii) in $H^{3}$ to generate the route set $\mathscr{N}$ of negative reduced cost routes setting $(\tilde{\boldsymbol{u}}, \tilde{\boldsymbol{v}}, \tilde{\boldsymbol{g}})=\left(\boldsymbol{u}^{\prime}, \boldsymbol{v}^{\prime}, \boldsymbol{g}^{\prime}\right)$ and $\gamma=-\varepsilon$; (iii) at Step 2 of the exact method (see $\S 4.4$ ) to generate the route set $\mathscr{R}^{3}$ setting $(\tilde{\boldsymbol{u}}, \tilde{\boldsymbol{v}}, \tilde{\boldsymbol{g}})=\left(\boldsymbol{u}^{\mathbf{3}}, \boldsymbol{v}^{\mathbf{3}}, \boldsymbol{g}^{\mathbf{3}}\right)$ and $\gamma=z_{U B}-L B_{3}$.

\subsubsection{Procedure GenP.}

Procedure GenP is Phase 1 of GenR and computes the path sets $\mathscr{F}$ and $\mathscr{B}$ using bounding functions based on the $n g$-path relaxation. 
In generating the set $\mathscr{B}$, GenP imposes that any path $B \in \mathscr{B}$ is undominated by any other path $B^{\prime} \in \mathscr{B}$ such that $V(B)=V\left(B^{\prime}\right), \sigma(B)=\sigma\left(B^{\prime}\right), c(B) \geq c\left(B^{\prime}\right)$, and $t(B) \leq t\left(B^{\prime}\right)$.

Let $\tilde{l b}(B)$ be a lower bound on the reduced cost $\tilde{c}_{\ell}$, with respect to $(\tilde{\boldsymbol{u}}, \tilde{\boldsymbol{v}}, \tilde{\boldsymbol{g}})$, of any route $\ell$ containing path $B$. Procedure GenP is a Dijkstra-like algorithm (see Baldacci et al. [2008]) generating $\mathscr{B}$ as a sequence of undominated paths $\left(B^{1}, \ldots, B^{h}\right)$, with $h \leq \Delta(\mathscr{B})$, such that $\tilde{l} b\left(B^{1}\right) \leq \ldots \leq \tilde{l b}\left(B^{h}\right) \leq \gamma$, where $\Delta(\mathscr{B})$ is a parameter. To compute $\tilde{l b}(B)$ we have to consider two cases

Case 1: $\tilde{\boldsymbol{g}}=\mathbf{0}$ (i.e., $\mathscr{C}=\varnothing$ ), functions $f(N G, t, i)$ are computed with the modified costs $\tilde{d}_{i j}$ and the subsets $N_{i}, i \in V$, contain the $\Delta\left(N_{i}\right)$ nearest customers to $i$ according to $\tilde{d}_{i j}$. Value $\tilde{l b}(B)$ is given by

$$
\tilde{l b}(B)=\tilde{c}(B)+\min _{N G \subseteq N_{i}: N G \cap V(B)=\left\{\sigma_{B}\right\}, t^{\prime} \leq t_{B}}\left\{f\left(N G, t^{\prime}, \sigma_{B}\right)\right\} .
$$

Case 2: $\tilde{\boldsymbol{g}} \neq \mathbf{0}, \tilde{l b}(B)$ is computed extending the $n g$-route relaxation to consider the dual variables $\tilde{\boldsymbol{g}}$. We define $N_{i}=\bigcup_{C \in \mathscr{C}: i \in C} C, i \in V$, and, for all $i \in V$ such that $\left|N_{i}\right|<\Delta\left(N_{i}\right)$, we add to $N_{i}$ the $\Delta\left(N_{i}\right)-\left|N_{i}\right|$ nearest customers to $i$ according to $\tilde{d}_{i j}$ not in $N_{i}$. We derive an expanded state-space graph $\widetilde{\mathscr{G}}_{F}=$ $\left(\widetilde{\mathscr{F}}, \widetilde{\mathscr{A}_{F}}\right)$ from $\widehat{\mathscr{G}}_{F}$ by partitioning all paths represented by state $(N G, t, i) \in \widehat{\mathscr{F}}$ in $\left|\Gamma_{i}^{-1}\right|$ partitions, where each partition is identified by the last vertex $j$ visited before $i$ and is represented by a state $(N G, t, j, i) \in \widetilde{\mathscr{F}}$. The sets $\widetilde{\mathscr{F}}$ and $\widetilde{\mathscr{A}_{F}}$ are defined as follows

$$
\begin{gathered}
\widetilde{\mathscr{F}}=\left\{(N G, t, j, i): i \in V, j \in \Gamma_{i}^{-1}, t \in\left[e_{i}, l_{i}\right], \forall N G \subseteq N_{i} \text { s.t. } N G \ni i\right\}, \\
\widetilde{\mathscr{A}_{F}}=\left\{\left(\left(N G^{\prime}, t^{\prime}, k, j\right),(N G, t, j, i)\right):\left(N G^{\prime}, t^{\prime}, k, j\right),(N G, t, j, i) \in \widetilde{\mathscr{F}},\right. \\
\left.j \in \Gamma_{i}^{-1}, k \in \Gamma_{j}^{-1}, t^{\prime} \in \Omega(t, j, i), \forall N G^{\prime} \subseteq N_{j} \text { s.t. } N G^{\prime} \ni j \text { and } N G^{\prime} \cap N_{i}=N G \backslash\{i\}\right\},
\end{gathered}
$$

where $\Omega(t, j, i)$ is the set of departure times from vertex $j$ to arrive at vertex $i$ at time $t$ when $j$ is visited immediately before $i$ and is defined as

$$
\Omega(t, j, i)= \begin{cases}\left.t^{\prime}: t^{\prime} \in\left[e_{j}, \min \left\{l_{j}, t-t_{j i}\right\}\right]\right\}, & \text { if } t=e_{i}, \\ t-t_{j i}: t-t_{j i} \in\left[e_{j}, l_{j}\right], & \text { if } t \in\left(e_{i}, l_{i}\right] .\end{cases}
$$

Let $\mathscr{C}_{i j}=\{C \in \mathscr{C}:(i, j) \in A(C)\}$. A possible DP recursion for computing the cost $f(N G, t, j, i)$ of a least cost $n g$-path $(N G, t, j, i) \in \widetilde{\mathscr{F}}$ is

$$
f(N G, t, j, i)=\min _{\substack{\left(N G^{\prime}, t^{\prime}, k, j\right): \\\left(\left(N G^{\prime}, t^{\prime}, k, j\right),(N G, t, j, i)\right) \in \widehat{A}_{F}}}\left\{f\left(N G^{\prime}, t^{\prime}, k, j\right)+\tilde{d}_{j i}-\sum_{C \in \mathscr{C}_{j i} \backslash \mathscr{C}_{k j}} \tilde{g}_{C}\right\} .
$$

The following lemma gives a method for computing $\tilde{l b}(B)$. 
Lemma 2. Let $i=\sigma_{B}$ and $k=\pi_{B}$. Lower bound $\tilde{l b}(B)$ can be computed as follows

$$
\tilde{l b}(B)=\tilde{c}(B)-\sum_{\substack{C \in \mathscr{C}: \\|A(C) \cap A(B)| \geq 1}} \tilde{g}_{C}+\min _{\substack{N G \subseteq N_{i}: N G \cap V(B)=\{i\}, t^{\prime} \leq t(B), j \in \Gamma_{i}^{-1}}}\left\{f\left(N G, t^{\prime}, j, i\right)+\tilde{g}_{\{j i k\}}\right\},
$$

where $\tilde{g}_{\{j i k\}}$ is the dual of inequality (4.6) corresponding to $C=\{j, i, k\}$ $\left(\tilde{g}_{\{j i k\}}=0\right.$ if $\left.\{j, i, k\} \notin \mathscr{C}\right)$.

Proof. Let $R$ be the route of minimum reduced cost with respect to $(\tilde{\boldsymbol{u}}, \tilde{\boldsymbol{v}}, \tilde{\boldsymbol{g}})$ containing the backward path $B$, and let $F$ be the forward path producing $R$ once combined with $B$. Let $\mathscr{W}(F), \mathscr{W}(B)$ and $\mathscr{W}(R)$ be the subsets of the WSR3s of $\mathscr{C}$ covered by $F, B$ and $R$, respectively. Notice that $\mathscr{W}(F) \cap \mathscr{W}(B)$ is either empty or contains the WSR3 inequality $C^{*} \in \mathscr{C}$ such that $A\left(C^{*}\right)$ contains both the first arc $(i, k)$ of $B$ and the terminal arc $(\pi(P), i)$ of $F$, that is $C^{*}=\{\pi(P), i, k\}$. If $\{\pi(P), i, k\} \in \mathscr{C}$, let $\tilde{g}_{\{\pi(P), i, k\}}$ be the dual of inequality (4.6) corresponding to $\{\pi(P), i, k\}$; otherwise $(\{\pi(P), i, k\} \notin \mathscr{C})$, set $\tilde{g}_{\{\pi(P), i, k\}}=0$. Because

$$
\tilde{c}(R)=\tilde{c}(F)+\tilde{c}(B)-\sum_{C \in \mathscr{W}(R)} \tilde{g}_{C}
$$

and

$$
\sum_{C \in \mathscr{W}(R)} \tilde{g}_{C}=\sum_{C \in \mathscr{W}(F)} \tilde{g}_{C}+\sum_{C \in \mathscr{W}(B)} \tilde{g}_{C}-\tilde{g}_{\{\pi(F), i, k\}}
$$

we have

$$
\tilde{c}(R)=\tilde{c}(B)-\sum_{C \in \mathscr{W}(B)} \tilde{g}_{C}+\tilde{c}(F)-\sum_{C \in \mathscr{W}(F)} \tilde{g}_{C}+\tilde{g}_{\{\pi(F), i, k\}} .
$$

Notice that

$$
\min _{\substack{N G \subseteq N_{i} \text { s.t. } N G \cap V(B)=\{i\}, t^{\prime} \leq t(B), j \in \Gamma_{i}^{-1}}}\left\{f\left(N G, t^{\prime}, j, i\right)+\tilde{g}_{\{j, i, k\}}\right\} \leq \tilde{c}(F)-\sum_{C \in \mathscr{W}(F)} \tilde{g}_{C}+\tilde{g}_{\{\pi(P), i, k\}} .
$$

From (4.19) and (4.20), we derive that $\tilde{l b}(B)$ computed according to expression (4.18) provides a valid lower bound on $\tilde{c}(R)$.

Similarly, GenP generates the path set $\mathscr{F}$ as a sequence of undominated paths $\left(F^{1}\right.$, $\left.\ldots, F^{h}\right)$, with $h \leq \Delta(\mathscr{F})$, such that $\tilde{l b}\left(F^{1}\right) \leq \ldots \leq \tilde{l b}\left(F^{h}\right) \leq \gamma$, where $\Delta(\mathscr{F})$ is a parameter. Bound $\tilde{l b}(F)$ is computed similarly as described above for $\mathscr{B}$ using the reverse functions $f^{-1}(N G, t, i)$ and $f^{-1}(N G, t, j, i)$. 


\subsubsection{Procedure Combine.}

This procedure combines the path sets $\mathscr{F}$ and $\mathscr{B}$ to derive at most $\Delta$ routes of reduced cost with respect to $(\tilde{\boldsymbol{u}}, \tilde{\boldsymbol{v}}, \tilde{\boldsymbol{g}})$ less than or equal to $\gamma$ using the iterative method described by Baldacci et al. [2006].

Because of Lemma 1, routes of reduced cost less than or equal to $\gamma$ can be generated combining only path pairs $(F, B)$ such that $\tilde{c}(F)+\tilde{c}(B) \leq \gamma$. Procedure Combine dynamically generates a sequence of pairs $\left(F^{r_{1}}, B^{s_{1}}\right), \ldots,\left(F^{r_{k}}, B^{s_{k}}\right), \ldots,\left(F^{r_{h}}, B^{s_{h}}\right)$ such that each pair satisfies conditions (4.12) and $\tilde{c}\left(F^{r_{1}}\right)+\tilde{c}\left(B^{s_{1}}\right) \leq \ldots \leq \tilde{c}\left(F^{r_{k}}\right)+$ $\tilde{c}\left(B^{s_{k}}\right) \leq \ldots \leq \tilde{c}\left(F^{r_{h}}\right)+\tilde{c}\left(B^{s_{h}}\right) \leq \gamma$. The pool of routes generated by Combine contains any route $R$ resulting from the pairs of paths in the sequence such that $\tilde{c}(R) \leq \gamma$ and $R$ is undominated by any other route $R^{\prime}$ previously generated (i.e., $R^{\prime}$ dominates $R$ if $V\left(R^{\prime}\right)=V(R)$ and $\left.\tilde{c}\left(R^{\prime}\right) \leq \tilde{c}(R)\right)$.

The procedure terminates as soon as $\Delta$ routes have been found or all pairs have been considered.

\subsubsection{GenR for the CVRP.}

Unlike the VRPTW, the path sets $\mathscr{F}$ and $\mathscr{B}$ are defined as in Baldacci et al. [2008]: $\mathscr{F}$ contains any forward path $F$ such that $q_{F} \leq \frac{Q}{2}+q_{\sigma_{F}}$ and $\mathscr{B}$ contains any backward path $B$ such that $q_{B} \leq \frac{Q}{2}+q_{\sigma_{B}}$ (for symmetric CVRPs $\mathscr{F}$ and $\mathscr{B}$ coincide).

Moreover, the lower bound $\tilde{l} b(B)$ is computed with bounding functions $f(N G, q, i)$, if $\tilde{\boldsymbol{g}}=\mathbf{0}$, or $f(N G, q, j, i)$, if $\tilde{\boldsymbol{g}} \neq \mathbf{0}$. Functions $f(N G, q, j, i)$ are derived by expanding $f(N G, q, i)$ so that $j$ is the vertex preceding $i$ similarly as described in $\S 4.5 .5 .1$ for functions $f(N G, t, j, i)$.

\subsection{Column-and-Cut Generation Procedure $H^{4}$}

Procedure $H^{4}$ is a column-and-cut generation procedure based on the simplex algorithm to solve relaxation $L P . H^{4}$ differs from the methods of Jepsen et al. [2008], Desaulniers et al. [2008], and Baldacci et al. [2008] for the pricing algorithm.

Before starting $H^{4}$ (see Step 2 of the exact algorithm in $\S 4.4$ ) an attempt is made to generate the set $\mathscr{R}^{3}$ of all routes such that $c_{\ell}^{3} \leq z_{U B}-L B_{3}$. The set $\mathscr{R}^{3}$ is generated by procedure GenR imposing that $|\mathscr{F}| \leq \Delta(\mathscr{F}),|\mathscr{B}| \leq \Delta(\mathscr{B})$, and that at most $\Delta(\mathscr{R})$ are generated. We call $\mathscr{F}^{3}$ the set $\mathscr{F}, \mathscr{B}^{3}$ the set $\mathscr{B}$, and $\mathscr{R}^{3}$ the set $\mathscr{R}$ generated by GenR. Define each set $\mathscr{F}^{3}, \mathscr{B}^{3}$, and $\mathscr{R}^{3}$ as optimal if $\left|\mathscr{F}^{3}\right|<\Delta(\mathscr{F}),\left|\mathscr{B}^{3}\right|<\Delta(\mathscr{B})$, and $\left|\mathscr{R}^{3}\right|<\Delta(\mathscr{R})$. The set $\mathscr{R}^{3}$ is optimal if and only if both $\mathscr{F}^{3}$ and $\mathscr{B}^{3}$ are optimal. 
The route set $\mathscr{R}^{\prime}$ of the initial master problem of $H^{4}$ is obtained by extracting the $\Delta^{a}$ routes of minimum reduced cost with respect to $\left(\boldsymbol{u}^{\mathbf{3}}, \boldsymbol{v}^{\mathbf{3}}, \boldsymbol{g}^{\mathbf{3}}\right)$ from $\mathscr{R}^{3}$ and by adding all single-customer routes. We initialize the set $\mathscr{C}$ of SR3s as $\mathscr{C}=\varnothing$ and use the same set $\mathscr{S}$ of SCs of $H^{3}$.

At each iteration, a set $\mathscr{N}$ of at most $\Delta^{b}$ negative reduced cost routes with respect to the current dual solution $(\overline{\boldsymbol{u}}, \overline{\boldsymbol{v}}, \overline{\boldsymbol{g}})$ is produced by procedure GenR4 (see $\S 4.6 .1)$. The set $\mathscr{N}$ is either extracted from $\mathscr{R}^{3}$ or generated by combining sets $\mathscr{F}^{3}$ and $\mathscr{B}^{3}$. The sets $\mathscr{F}^{3}$ and $\mathscr{B}^{3}$ are newly generated when necessary if they are not-optimal.

At each iteration, $H^{4}$ adds the set $\mathscr{C}^{\prime}$ of at most $\Delta(\mathscr{C})$ SR3s most violated by the current $L P$ solution. $H^{4}$ ends if $\mathscr{N}=\varnothing$ and $\mathscr{C}^{\prime}=\varnothing$ and achieves a $D$ solution $\left(\boldsymbol{u}^{\mathbf{4}}, \boldsymbol{v}^{\mathbf{4}}, \boldsymbol{g}^{\mathbf{4}}\right)$ of cost $L B_{4}$.

\subsubsection{Procedure GenR4}

Procedure GenR4 is called at each iteration of $H^{4}$ to generate the route set $\mathscr{N}$ of at most $\Delta^{b}$ routes of negative reduced cost with respect to the current dual solution $(\overline{\boldsymbol{u}}, \overline{\boldsymbol{v}}, \overline{\boldsymbol{g}})$ of the master problem.

GenR4 performs the following steps

Step 1. Extract from $\mathscr{R}^{3}$ a set of at most $\Delta^{b}$ routes of negative reduced cost and add them to $\mathscr{N}$. If $\mathscr{N} \neq \varnothing$ or $\mathscr{R}^{3}$ is optimal, stop.

Step 2. Call procedure Combine4 that combines the sets $\mathscr{F}^{3}$ and $\mathscr{B}^{3}$ to derive the set $\mathscr{N}$. If $\mathscr{N} \neq \varnothing$ or both sets $\mathscr{F}^{3}$ and $\mathscr{B}^{3}$ are optimal, stop.

Step 3. If $\mathscr{F}^{3}$ is not-optimal, call procedure GenP4 to generate a new path set $\mathscr{F}$ and set $\mathscr{F}^{3}=\mathscr{F}\left(\mathscr{F}^{3}\right.$ is still not-optimal).

Step 4. Similarly to the previous step, if $\mathscr{B}^{3}$ is not-optimal, call procedure GenP4 to generate a new path set $\mathscr{B}$ and set $\mathscr{B}^{3}=\mathscr{B}\left(\mathscr{B}^{3}\right.$ is still not-optimal $)$.

Step 5. Call Combine4 that combines the sets $\mathscr{F}^{3}$ and $\mathscr{B}^{3}$ to derive the set $\mathscr{N}$.

If GenP4 or Combine4 run out of memory, $H^{4}$ and the exact method terminate prematurely.

\subsubsection{Procedure GenP4}

Procedure GenP4 generates sets $\mathscr{F}$ and/or $\mathscr{B}$ in Steps 3 and 4 of GenR4. GenP4 is similar to procedure GenP but applies different fathoming rules and an additional dominance 
rule introduced by Jepsen et al. [2008]. In generating $\mathscr{B}$, procedure GenP4 applies the following rules.

Let $l b^{3}(B), B \in \mathscr{B}$, be a lower bound to the reduced cost with respect to the dual solution $\left(\boldsymbol{u}^{\mathbf{3}}, \boldsymbol{v}^{\mathbf{3}}, \boldsymbol{g}^{\mathbf{3}}\right)$ found by $H^{3}$ of any route $R$ containing path $B$. Lower bound $l b^{3}(B)$ corresponds to $\tilde{l b}(B)$ described in $§ 4.5 .5 .1$ when $(\tilde{\boldsymbol{u}}, \tilde{\boldsymbol{v}}, \tilde{\boldsymbol{g}})$ is replaced with $\left(\boldsymbol{u}^{\mathbf{3}}, \boldsymbol{v}^{\mathbf{3}}, \boldsymbol{g}^{\mathbf{3}}\right)$.

Fathoming 1 . Any path $B \in \mathscr{B}$ such that $l b^{3}(B)>z_{U B}-L B_{3}$ can be fathomed because $B$ cannot generate any route of any VRPTW solution of cost less than or equal to $z_{U B}$.

Let $\overline{l b}(B), B \in \mathscr{B}$, be a lower bound to the reduced cost with respect to the dual solution $(\overline{\boldsymbol{u}}, \overline{\boldsymbol{v}}, \overline{\boldsymbol{g}})$ of any route containing path $B$.

Fathoming 2. Any path $B$ such that $\overline{l b}(B) \geq 0$ is fathomed.

Lower bound $\overline{l b}(B)$ can be computed as described in the following lemma.

Lemma 3. Let functions $f(N G, t, i)$ be computed with the arc costs $\bar{d}_{i j}=d_{i j}-\frac{1}{2}\left(\bar{u}_{i}+\right.$ $\left.\bar{u}_{j}\right)-\sum_{S \in \mathscr{S}_{i j}} \bar{v}_{S}$. Let $\bar{c}(B)$ be the cost of path $B$ using arc costs $\bar{d}_{i j}$, and let $i=\sigma_{B}$. Lower bound $\overline{l b}(B)$ can be computed as follows

$$
\overline{l b}(B)=\bar{c}(B)-\sum_{\substack{C \in \mathscr{C} \\
|C \cap V(B) \backslash\{i\}| \geq 2}} \bar{g}_{C}+\min _{\begin{array}{c}
N G \subseteq N_{i}: \\
N G \cap V(B)=\{i\}, t^{\prime} \leq t_{B}
\end{array}}\left\{f\left(N G, t^{\prime}, i\right)-\sum_{\begin{array}{c}
C \in \mathscr{C} \\
|C \cap N G| \geq 2
\end{array}} \bar{g}_{C}\right\} .
$$

Proof. Let $R$ be the route of minimum reduced cost with respect to $(\overline{\boldsymbol{u}}, \overline{\boldsymbol{v}}, \overline{\boldsymbol{g}})$ containing the backward path $B$, and let $F$ be the forward path producing $R$ once combined with $B$. Let $\mathscr{C}(S)=\{C \in \mathscr{C}:|C \cap S| \geq 2\}$. We have

$$
\bar{c}(R)=\bar{c}(B)-\sum_{C \in \mathscr{C}(V(B) \backslash\{i\})} \bar{g}_{C}+\bar{c}(F)-\sum_{C \in \mathscr{C}(V(F))} \bar{g}_{C}-\sum_{C \in \overline{\mathscr{C}}(V(R))} \bar{g}_{C}
$$

where $\overline{\mathscr{C}}(V(R))=\mathscr{C}(V(R)) \backslash(\mathscr{C}(V(B) \backslash\{i\}) \cup \mathscr{C}(V(F)))$. Because $\overline{\boldsymbol{g}} \leq \mathbf{0}$ and

$$
\min _{N G \subseteq N_{i}: N G \cap V(B)=\{i\},}\left\{f\left(N G, t^{\prime}, i\right)-\sum_{\substack{C \in \mathscr{C} ; \\ t^{\prime} \leq t_{B} \\|C \cap N G| \geq 2}} \bar{g}_{C}\right\} \leq \bar{c}(F)-\sum_{C \in \mathscr{C}(V(F))} \bar{g}_{C},
$$

we have

$$
\bar{c}(R) \geq \bar{c}(B)-\sum_{C \in \mathscr{C}(V(B) \backslash\{i\})} \bar{g}_{C}+\min _{\begin{array}{c}
N G \subseteq N_{i}: \\
N G \cap V(B)=\{i\}, t^{\prime} \leq t_{B}
\end{array}}\left\{f\left(N G, t^{\prime}, i\right)-\sum_{\begin{array}{c}
C \in \mathscr{C} \\
|C \cap N G| \geq 2
\end{array}} \bar{g}_{C}\right\},
$$

thus proving that expression (4.21) provides a valid lower bound $\overline{l b}(B)$ on $\bar{c}(R) \cdot \square$ The dominance rule of Jepsen et al. [2008] when applied to $\mathscr{B}$ is as follows. 
Dominance 1. Let $B, B^{\prime} \in \mathscr{B}$ be two backward paths such that $\sigma_{B^{\prime}}=\sigma_{B}, t_{B^{\prime}} \geq t_{B}$, $q_{B^{\prime}} \geq q_{\text {min }}, q_{B} \geq q_{\text {min }}$, and $V\left(B^{\prime}\right) \subseteq V(B)$. Path $B^{\prime}$ dominates path $B$ if

$$
\bar{c}\left(B^{\prime}\right) \leq \bar{c}(B)-\sum_{C \in \mathscr{C}:\left|C \cap V(B) \backslash V\left(B^{\prime}\right)\right| \geq 2} \bar{g}_{C}
$$

Similar rules are applied by procedure GenP4 to generate the path set $\mathscr{F}$.

\subsubsection{Procedure Combine4}

This procedure generates the route set $\mathscr{N}$ of negative reduced costs with respect to the dual solution $(\overline{\boldsymbol{u}}, \overline{\boldsymbol{v}}, \overline{\boldsymbol{g}})$ of the master problem combining the path sets $\mathscr{F}^{3}$ and $\mathscr{B}^{3}$ as defined in $\S 4.6 .1$. Procedure Combine4 corresponds to procedure Combine (see $\S 4.5 .5 .2)$ replacing $(\tilde{\boldsymbol{u}}, \tilde{\boldsymbol{v}}, \tilde{\boldsymbol{g}})$ with $(\overline{\boldsymbol{u}}, \overline{\boldsymbol{v}}, \overline{\boldsymbol{g}})$, setting $\gamma=-\varepsilon$, and applying the following fathoming rule to reduce the set $\mathscr{N}$.

Fathoming 3. Let $c^{3}(R)$ be the reduced cost of route $R$ with respect to $\left(\boldsymbol{u}^{\mathbf{3}}, \boldsymbol{v}^{\mathbf{3}}, \boldsymbol{g}^{\mathbf{3}}\right)$ computed as $c^{3}(R)=c(R)-\sum_{i \in V(R)} u_{i}^{3}-\sum_{S \in \mathscr{S}} \rho_{\ell}(S) v_{S}^{3}-\sum_{C \in \mathscr{C}:|A(C) \cap A(R)| \geq 1} g_{C}^{3}$. A route $R$ of negative reduced cost with respect to $(\overline{\boldsymbol{u}}, \overline{\boldsymbol{v}}, \overline{\boldsymbol{g}})$ cannot belong to $\mathscr{N}$ if $c^{3}(R)>z_{U B}-L B_{3}$ as route $R$ cannot be in any solution of cost less than or equal to $z_{U B}$.

\subsubsection{Procedure GenR4 for the CVRP}

The lower bound $l b^{3}(B)$ is computed using bounding functions $f(N G, q, j, i)$ as described in $\S 4.5 .5 .3$ for $\tilde{l} b(B)$ whereas the lower bound $\overline{l b}(B)$ is computed according to expression (4.21) but using bounding functions $f(N G, q, i)$ instead of $f(N G, t, i)$.

\subsection{Procedure GenRF for Generating Route Set $\widetilde{R}$}

The exact method described in $\S 4.4$ at Step 4 asks to generate the largest subset $\widetilde{\mathscr{R}} \subseteq \mathscr{R}$ of routes satisfying conditions (4.8), whenever $\mathscr{R}^{3}$ is not-optimal.

For this purpose, we use a two-phase procedure, called GenRF, similar to GenR (see $\S 4.5 .5)$. In the first phase, two sets $\mathscr{F}$ and $\mathscr{B}$ of forward and backward paths are computed as described below. In the second phase, these sets are combined by procedure CombineF (see $\S 4.7 .2$ ) to derive the final route set $\widetilde{\mathscr{R}}$. In the first phase, there are four cases

Case 1. Both $\mathscr{F}^{3}$ and $\mathscr{B}^{3}$ generated by GenR are optimal. We set $\mathscr{F}=\mathscr{F}^{3}$ and $\mathscr{B}=\mathscr{B}^{3}$. 
Case 2. $\mathscr{B}^{3}$ is optimal but $\mathscr{F}^{3}$ is not. We set $\mathscr{B}=\mathscr{B}^{3}$ and call GenPF (§4.7.1) to compute $\mathscr{F}$.

Case 3. $\mathscr{F}^{3}$ is optimal but $\mathscr{B}^{3}$ is not. We set $\mathscr{F}=\mathscr{F}^{3}$ and call GenPF to compute $\mathscr{B}$.

Case 4. Both $\mathscr{F}^{3}$ and $\mathscr{B}^{3}$ are not optimal. We call GenPF to compute both $\mathscr{F}$ and $\mathscr{B}$.

In the end, $\widetilde{\mathscr{R}}$ is defined optimal (i.e., contains any optimal VRPTW solution) if and only if $\mathscr{F}, \mathscr{B}$, and $\widetilde{\mathscr{R}}$ are such that $|\mathscr{F}|<\Delta(\mathscr{F}),|\mathscr{B}|<\Delta(\mathscr{B})$ and $|\widetilde{\mathscr{R}}|<\Delta(\widetilde{\mathscr{R}})$.

In the following $\S \S 4.7 .1$ and 4.7.2, we describe procedure GenPF and CombineF for the VRPTW. It is quite obvious how to adapt them for the CVRP.

\subsubsection{Procedure GenPF}

Procedure GenPF generates one or both sets $\mathscr{F}$ and $\mathscr{B}$ required by GenRF (see cases $(2)$, (3) and (4) in $\S 4.7)$. GenPF is similar to procedure GenP but applies different fathoming rules.

In generating the set $\mathscr{B}$, GenPF applies the following fathoming rules. Let $l b^{3}(B)$ and $l b^{4}(B)$ be the lower bounds on the reduced costs, $c^{3}(R)$ and $c^{4}(R)$, of any route $R$ containing path $B \in \mathscr{B}$ with respect to $\left(\boldsymbol{u}^{\mathbf{3}}, \boldsymbol{v}^{\mathbf{3}}, \boldsymbol{g}^{\mathbf{3}}\right)$ and $\left(\boldsymbol{u}^{\mathbf{4}}, \boldsymbol{v}^{\mathbf{4}}, \boldsymbol{g}^{\mathbf{4}}\right)$, respectively. Bound $l b^{3}(B)$ is computed as described in $\S 4.6 .2$, and $l b^{4}(B)$ is computed using expression (4.21) where $(\overline{\boldsymbol{u}}, \overline{\boldsymbol{v}}, \overline{\boldsymbol{g}})$ is replaced with $\left(\boldsymbol{u}^{\mathbf{4}}, \boldsymbol{v}^{\mathbf{4}}, \boldsymbol{g}^{\mathbf{4}}\right)$.

Fathoming 4. Any path $B \in \mathscr{B}$ such that $l b^{3}(B)>z_{U B}-L B_{3}$ or $l b^{4}(B)>z_{U B}-L B_{4}$ can be fathomed.

Fathoming 5. Let $d_{i j}^{4}=d_{i j}-\frac{1}{2}\left(u_{i}^{4}+u_{j}^{4}\right)-\sum_{S \in \mathscr{S}_{i j}} v_{s}^{4},(i, j) \in A$. Whenever $q_{\text {min }}=0$, any backward path $B$ such that

$$
\sum_{(i, j) \in A(B)} d_{i j}^{4}-\sum_{C \in \mathscr{C}:|C \cap V(B) \backslash\{i\}| \geq 2} g_{C}^{4}-d_{i 0}^{4}>z_{U B}-L B_{4}
$$

can be fathomed as it cannot produce any route $R$ of reduced cost $c^{4}(R) \leq z_{U B}-L B_{4}$.

Proof. Let $R$ be the route of minimum reduced cost $c^{4}(R)$ with respect to $\left(\boldsymbol{u}^{\mathbf{4}}, \boldsymbol{v}^{\mathbf{4}}, \boldsymbol{g}^{\mathbf{4}}\right)$ obtained by combining $B$ starting from $\sigma_{B}=i$ with a forward path $F$ ending at vertex $\sigma_{F}=i$. Let $\mathscr{C}(S)=\{C \in \mathscr{C}:|C \cap S| \geq 2\}$. We have

$$
c^{4}(R)=\sum_{(i, j) \in A(R)} d_{i j}^{4}-\sum_{C \in \mathscr{C}(V(R))} g_{C}^{4} .
$$

Since $\mathscr{C}(V(R)) \supseteq \mathscr{C}(V(B) \backslash\{i\}) \cup \mathscr{C}(V(F))$ and $g_{C}^{4} \leq 0, C \in \mathscr{C}$, we have

$$
c^{4}(R) \geq \sum_{(i, j) \in A(B)} d_{i j}^{4}-\sum_{C \in \mathscr{C}(V(B) \backslash\{i\})} g_{C}^{4}+\sum_{(i, j) \in A(F)} d_{i j}^{4}-\sum_{C \in \mathscr{C}(V(F))} g_{C}^{4} .
$$


Consider the route $R^{\prime}$ obtained by adding arc $(i, 0)$ to $F$. As $c^{4}\left(R^{\prime}\right) \geq 0$, we have

$$
\sum_{(i, j) \in A(F)} d_{i j}^{4}-\sum_{C \in \mathscr{C}(V(F))} g_{C}^{4} \geq-d_{i 0}^{4} .
$$

From inequalities (4.23) and (4.24), we obtain

$$
c^{4}(R) \geq \sum_{(i, j) \in A(B)} d_{i j}^{4}-\sum_{C \in \mathscr{C}(V(B) \backslash\{i\})} g_{C}^{4}-d_{i 0}^{4} .
$$

Thus, if $B$ satisfies inequality (4.22), then $c^{4}(R)>z_{U B}-L B_{4}$. So route $R$ cannot be in any optimal solution of cost less than or equal to $z_{U B}$.

Similar fathoming rules are used to generate $\mathscr{F}$.

\subsubsection{Procedure CombineF}

This procedure generates the route set $\widetilde{R}$ combining the path sets $\mathscr{F}$ and $\mathscr{B}$ defined in $\S 4$.7. CombineF is similar to Combine (see $\S 4.5 .5 .2)$ but imposes that any route $R \in \widetilde{\mathscr{R}}$ is such that $c^{3}(R) \leq z_{U B}-L B_{3}$ and $c^{4}(R) \leq z_{U B}-L B_{4}$.

\subsection{Computational Results}

This section reports on the computational results of the exact method (hereafter called BMR) described in this chapter. All algorithms were coded in Fortran 77 and compiled with Intel Fortran 11.0. Cplex 12.1 was used as the LP solver in procedure $H^{4}$ and the IP solver in the exact method. All tests were run on an IBM Intel Xeon X7350 Server $(2.93 \mathrm{GHz}-16 \mathrm{~GB}$ of RAM).

\subsubsection{Computational Results on the VRPTW}

Our exact method BMR for the VRPTW was tested on Solomon instances Solomon [1987], which are divided into six classes (classes C1, RC1 and R1 with tight time windows and strict vehicle capacity, and classes $\mathrm{C} 2, \mathrm{RC} 2$ and $\mathrm{R} 2$ with wide time windows and loose vehicle capacity). We considered all 100-customer instances and instances with 50 customers of classes $\mathrm{C} 2, \mathrm{RC} 2$ and $\mathrm{R} 2$.

The travel costs $d_{i j}$ are computed as $d_{i j}=\left\lfloor 10 e_{i j}\right\rfloor / 10$, where $e_{i j}$ is the Euclidean distance between vertices $i$ and $j$; the travel times $t_{i j}$ are integer values computed as $t_{i j}=10\left(d_{i j}+s_{i}\right)$, where $s_{i}$ is the service time at vertex $i$. 
Because instances of classes $\mathrm{C} 1, \mathrm{RC} 1$ and $\mathrm{R} 1$ have tight time windows, we did not find it worth running procedure $H^{2}$. Thus, on such instances $H^{2}$ was skipped. In addition, we ignored SCs and WSR3s for all classes of instances.

BMR uses the best upper bounds reported in Ropke [2005] and Danna and Pape [2005]. Such upper bounds are obtained by running the heuristic of Pisinger and Ropke [2007] with different parameter settings (Ropke [2010]). Whenever BMR uses the upper bound, its computing time is added to the total computing time of BMR. For instance R211 with 100 customers, the upper bound used was found by Desaulniers et al. [2008].

BMR uses the following parameter setting

- in $H^{1}:$ Maxit $1=100$ and Maxit $2=50$;

- in $H^{2}: \Delta\left(N_{i}\right)=8$, Maxit $1=100$, and Maxit $2=50$;

- in $H^{3}: \Delta\left(N_{i}\right)=10, \Delta^{b}=300, \Delta(\mathscr{F})=\Delta(\mathscr{B})=5 \times 10^{7}, \Delta(\mathscr{R})=1.5 \times 10^{6}$, Maxit $1=100$, and Maxit $2=50$;

- in $H^{4}, \Delta^{a}=1 \times 10^{4}, \Delta^{b}=300, \Delta(\mathscr{F})=\Delta(\mathscr{B})=5 \times 10^{7}, \Delta(\mathscr{R})=1.5 \times 10^{6}$, $\Delta(\mathscr{C})=20$.

We compare BMR with the methods of Jepsen et al. [2008] and Desaulniers et al. [2008], hereafter called JPSP and DHL, respectively. Desaulniers et al. [2008] presented three versions of their algorithm. We consider the version labeled "ESPPRC SRC" as it could solve more instances than the others. According to SPEC (http://www.spec. org/benchmarks.html), our machine is three times faster than the Intel Pentium 4 3.0 GHz PC of JPSP and twice as fast as the Linux PC Dual Core AMD Opteron at $2.6 \mathrm{GHz}$ of DHL.

In Tables 4.1, 4.2, and 4.3, we report on detailed computational results on the Solomon instance considered. The columns of three tables report the instance name (Inst), the optimal value ( $z^{*}$ - in bold if solved for the first time by BMR), the upper bound used $\left(z_{U B}\right)$ and the time to compute it $(T)$ in columns under heading Upper Bound. For each bounding procedure $H^{k}, k=1, \ldots, 4$ (if run), we report the lower bound $\left(L B_{k}\right)$ and the cumulative computing time spent up to $H^{k}$. Columns $\left|\mathscr{F}^{3}\right|,\left|\mathscr{B}^{3}\right|$, and $\left|\mathscr{R}^{3}\right|$ report the cardinalities (in thousands) of the sets $\mathscr{F}^{3}, \mathscr{B}^{3}$ and $\mathscr{R}^{3}$; if we could not completely generate a set, an empty circle is displayed. The number of SR3s inequalities (SR3) added in $H^{4}$ is shown. The number of routes $(|\widetilde{\mathscr{R}}|)$ in the final reduced problem $\widetilde{P}$ and the time taken by Cplex to solve it $\left(T_{c p x}\right)$ are shown. Finally, the total computing time in seconds $\left(T_{t o t}\right)$ of the methods compared are reported in the last three columns of the table. $T_{\text {tot }}$ under BMR is equal to the sum of the time to compute the upper bound, the time spent up to $H^{4}$ and $T_{c p x}$.

Table 4.3 shows that BMR was able to solve four instances open so far. The only open Solomon instance is R.208.100, where BMR ran out of memory. Notice that the 
lower bounds achieved using the $n g$-routes in algorithm $C C G$ are close to the bounds achieved using elementary routes and the time taken to perform $H^{2}$ is limited.

Table 4.4 compares BMR, JPSP, and DHL. For each class, Table 4.4 reports the class name (Class), the number of customers $(n)$, the number of instances $(N P)$, the number of instances solved by each of the three methods (Solved) and the average computing time in seconds $(T)$ (n.a. means data are not available). In the last three rows, the average computing time of the methods over all instances, the instances solved by JPSP, and the instances solved by DHL are shown.

Table 4.4 shows that BMR outperforms JPSP and DHL: all instances solved by the other methods were solved by BMR and the average time is significantly lower.

Table 4.5 reports the optimal solutions BMR found for the four 100-customer instances (RC204, RC208, R204, and R211) open before this paper. We give the optimal solution value, the number of vehicles used, and, for each route, the cost and the sequence of visited customers.

We also report a computational analysis about some components of the algorithm proposed (i.e., parameter $\Delta\left(N_{i}\right)$ and dominance and fathoming rules). Table 4.6 reports the results obtained on a selected set of instances by varying parameter $\Delta\left(N_{i}\right)$ in procedure $H^{2}$. In particular, the table shows the value of lower bound $L B_{2}$ using $\Delta\left(N_{i}\right)=5,8,10,12$, and the corresponding computing time, compared with $L B_{1}, L B_{3}$, and $L B_{4}$. The last line of the table reports the average percentage deviations of the different lower bounds. The table shows that using $\Delta\left(N_{i}\right)=8$ gives a good trade-off between quality of the lower bound and computing time. Indeed, the lower bound is on average about 5 percent greater than $L B_{1}$ and about 0.5 percent lower than $L B_{3}$.

Tables 4.7 and 4.8 report statistics on the number of states fathomed by the dominance and fathoming rules applied in procedures GenP4 (called by procedure $H^{4}$ ) and GenPF (called to generate the final route sets $\widetilde{\mathscr{R}}$ ). The tables show the following columns: total number of forward (backwards) states in millions generated in computing $\mathscr{F}(\mathscr{B})$ (States), percentage of states eliminated by Dominance 1 (\%Dom 1$)$, percentage of states fathomed by Fathoming rule $x$ ( $\%$ Fath $x, x=1,2,4,5)$, final cardinality (in millions) of the set $\mathscr{F}(\mathscr{B})$ generated $(|\mathscr{F}|,|\mathscr{B}|)$. The dominance and fathoming rules are reported in the tables using the same order of application in BMR.

Regarding procedure GenP4, table 4.7 shows that the dominance and fathoming rules are very effective in reducing the number of states. Indeed, on average about $90 \%$ of the states generated (for both sets $\mathscr{F}$ and $\mathscr{B}$ ) are eliminated. In particular, the new Fathoming 1 and Fathoming 2 rules eliminates on average $80 \%$ of the states not dominated by Dominance 1 .

Concerning procedure GenPF, table 4.8 shows that both Fathoming 5 and 4 eliminate on average about $90 \%$ of the states generated. 


\subsubsection{Computational Results on the CVRP}

We considered classes A, B, E, F, M, and P available at http://branchandcut.org/ VRP/data. Cost $d_{i j}$ is an integer value computed as $d_{i j}=\left\lfloor e_{i j}+0.5\right\rfloor$, where $e_{i j}$ is the Euclidean distance between $i$ and $j$. As done by Fukasawa et al. [2006], we impose that exactly $m$ vehicles are used in the solution by simply transforming constraint (4.3) into an equality constraint.

For computational convenience we skip bounding procedure $H^{2}$, so the sequence of the bounding procedures is $H^{1}, H^{3}$, and $H^{4}$. WSR3s are used whenever $\lceil n / m\rceil \geq 12$.

If BMR cannot solve a problem in Step 1, we use the upper bound used by Fukasawa et al. [2006] and Baldacci et al. [2008], but, while generating the route set $\mathscr{R}^{3}$, whenever in GenP $\left|\mathscr{F}^{3}\right|>1 \times 10^{6}$, we run our implementation of the tabu search algorithm of Gendreau et al. [1994] with a time limit of 180 seconds. The computing time of the Tabu Search is considered in the total computing time of BMR whereas the computing time of the initial upper bound is ignored (as done by Fukasawa et al. [2006] and Baldacci et al. [2008]).

In our tests, we use the following parameter setting

- in $H^{1}$ and $H^{2}$ : Maxit $1=150$ and Maxit $2=100$;

- in $H^{3}: \Delta\left(N_{i}\right)=10, \Delta^{b}=300, \Delta(\mathscr{F})=1 \times 10^{7}, \Delta(\mathscr{R})=1 \times 10^{7}, \Delta(\mathscr{C})=100$, Maxit $1=150$, and Maxit $2=100$;

- in $H^{4}: \Delta^{a}=1 \times 10^{4}, \Delta^{b}=200, \Delta(\mathscr{F})=1 \times 10^{7}, \Delta(\mathscr{R})=1 \times 10^{6}, \Delta(\mathscr{C})=10$, Maxit $1=150$, and Maxit $2=100$.

BMR is compared with the methods of Baldacci et al. [2008] (BCM), Fukasawa et al. [2006] (FLL) and Lysgaard et al. [2004] (LLE). According to SPEC, our machine is three times faster than the Pentium $42.6 \mathrm{GHz} \mathrm{PC}$ of $\mathrm{BCM}$ and the Pentium 42.4 GHZ PC of FLL and ten times faster than the Intel Celeron $700 \mathrm{MHz}$ PC of LLE.

Tables 4.9, 4.10, 4.11, 4.12, and 4.13 show the same columns of Tables 4.1, 4.2, and 4.3 reported for the VRPTW. Column $z_{U B}$ reports the initial upper bound whereas column $U B_{4}$ reports the upper bound computed by our tabu search heuristic (when run). Under FLL, column $(s)$ indicates that the BCP algorithm based on s-cycle-free $q$-routes was used, whereas (-) indicates that the BC was used instead of the BCP. The total time of BMR is the sum of the time spent up to $H^{4}$ (that includes the time spent to compute $U B_{4}$ ) and $T_{c p x}$, but, if $H^{k}$, for some $k \in\{1,2,3\}$ succeeds in solving the problem to optimality, the time is equal to the time spent up to $H^{k}$.

Tables 4.9-4.13 show that BMR solves three problems not solved by BCM but does not solve problem F-n135-k7 that is solved by FLL using the BC algorithm. It is worth mentioning that problem F-n135-k7 was solved for the first time by Augerat [1995]. 
Table 4.14 summarizes the results of the methods on the six CVRP classes considered. For each class, the name (Class), the number of instances $(N P)$ and, for each exact method, the number of instances solved to optimality $(O p t)$, the average percentage deviation of the lower bound $(\% L B)$ and the average computing time in seconds $(T)$ are reported. Under FLL, column $O p t_{B C P}$ and $O p t_{B C}$ report the number of instances solved using $\mathrm{BCP}$ and $\mathrm{BC}$, respectively. The last two rows indicate average values of $\% L B$ and $T$ and the number of problems solved by each method.

Table 4.14 clearly shows that BMR outperforms the other methods on all classes but class F, where the BC algorithm of Lysgaard et al. [2004] outperforms the other methods.

Table 4.15 reports the results obtained by varying parameter $\Delta\left(N_{i}\right)$ in procedure $H^{2}$ and by using different types of inequalities in procedures $H^{1}$ and $H^{3}$ on a set of selected instances. For procedure $H^{1}$, the value of lower bound $L B_{1}$ and the corresponding computing time without (no CCs) and with (CCs) CCs are shown. The table shows the value of lower bound $L B_{2}$ using $\Delta\left(N_{i}\right)=5,8,10,12$, and the corresponding computing time. For procedure $H^{3}$, we show the value of lower bound $L B_{3}$ and the corresponding computing time without (no WSR3s) and with (WSR3s) WSR3s. The last line of the last reports the average percentage deviations of the different lower bounds.

The results show that: (i) increasing parameter $\Delta\left(N_{i}\right)$ slightly improves lower bound $L B_{2}$; (ii) $L B_{1}$ with CCs is very close to $L B_{3}$ without WSR3s; ( $i i i$ ) the increase of $L B_{2}$ with respect to $L B_{1}$ achieved by $H^{1}$ with CCs is very small and is not worth the extra computing time required by procedure $H^{2}$; (iv) CCs and WSR3s substantially increase lower bounds $L B_{1}$ and $L B_{3}$, respectively. It is worth mentioning that on instance En101-k14 lower bound $L B_{1}$ with CCs is greater than lower bound $L B_{2}$. This happens as 2 -vertex loops are allowed in the $n g$-route relaxation while they are forbidden in the $q$-route relaxation.

\subsection{Conclusions}

In this chapter, we described an exact method for solving the VRPTW and CVRP based on the set partitioning formulation strengthened with valid inequalities. We introduced a new route relaxation, called $n g$-route, that improves other nonelementary route relaxations proposed in the literature and a new strategy for solving the pricing problem in a column-and-cut generation procedure that involve the use of multiple dual solutions.

We reported computational results showing that the proposed method solves 4 of the 5 open Solomon VRPTW instances and is significantly faster than the state-of-the-art algorithms for both VRPTW and CVRP. 
TABLE 4.1: Detailed results on VRPTW Solomon instances with 50 customers and wide time windows

\begin{tabular}{|c|c|c|c|c|c|c|c|c|c|c|c|c|c|c|c|c|c|c|c|}
\hline \multirow[b]{2}{*}{ Inst } & \multirow[b]{2}{*}{$z^{*}$} & \multicolumn{2}{|c|}{ Upper Bound } & \multicolumn{2}{|c|}{ Proc. $H^{1}$} & \multicolumn{2}{|c|}{$\underline{\text { Proc. } H^{2}}$} & \multicolumn{2}{|c|}{ Proc. $H^{3}$} & \multirow[b]{2}{*}{$\left|\mathscr{F}^{3}\right|$} & \multirow[b]{2}{*}{$\left|\mathscr{B}^{3}\right|$} & \multirow[b]{2}{*}{$\left|\mathscr{R}^{3}\right|$} & \multicolumn{3}{|c|}{ Proc. $H^{4}$} & \multicolumn{2}{|c|}{ Problem $\widetilde{P}$} & \multicolumn{2}{|c|}{$T_{t o t}$} \\
\hline & & $z_{U B}$ & $T$ & $L B_{1}$ & $T$ & $L B_{2}$ & $T$ & $L B_{3}$ & $T$ & & & & $S R 3$ & $L B_{4}$ & $T$ & $|\widetilde{\mathscr{R}}|$ & $T_{c p x}$ & BMR & JPSP \\
\hline C. 201.50 & 360.2 & 360.2 & 83 & 360.2 & 3 & 360.2 & 4 & & & & & & & & & & & 4 & 42 \\
\hline C. 202.50 & 360.2 & 360.2 & 89 & 360.2 & 3 & 360.2 & 6 & & & & & & & & & & & 6 & 67 \\
\hline C. 203.50 & 359.8 & 359.8 & 89 & 359.8 & 3 & 359.8 & 7 & & & & & & & & & & & 7 & 214 \\
\hline C. 204.50 & 350.1 & 350.1 & 96 & 350.1 & 3 & 350.1 & 8 & & & & & & & & & & & 8 & - \\
\hline C. 205.50 & 359.8 & 359.8 & 99 & 358.2 & 4 & 359.8 & 7 & & & & & & & & & & & 7 & 64 \\
\hline C. 206.50 & 359.8 & 359.8 & 86 & 344.2 & 4 & 359.8 & 10 & & & & & & & & & & & 10 & 38 \\
\hline C. 207.50 & 359.6 & 359.6 & 89 & 356.6 & 4 & 359.6 & 9 & & & & & & & & & & & 9 & 72 \\
\hline C. 208.50 & 350.5 & 350.5 & 92 & 340.4 & 4 & 350.5 & 9 & & & & & & & & & & & 9 & 55 \\
\hline RC.201.50 & 684.8 & 684.8 & 40 & 670.1 & 3 & 684.8 & 8 & & & & & & & & & & & 8 & 3 \\
\hline RC.202.50 & 613.6 & 613.6 & 40 & 504.0 & 3 & 613.5 & 6 & 613.6 & 7 & & & & & & & & & 7 & 10 \\
\hline RC.203.50 & 555.3 & 555.3 & 50 & 409.2 & 3 & 555.3 & 15 & & & & & & & & & & & 15 & 190 \\
\hline RC.204.50 & 444.2 & 444.2 & 63 & 314.4 & 3 & 444.2 & 27 & & & & & & & & & & & 27 & - \\
\hline RC.205.50 & 630.2 & 630.2 & 40 & 541.4 & 3 & 630.2 & 9 & & & & & & & & & & & 9 & 5 \\
\hline RC.206.50 & 610.0 & 610.0 & 43 & 441.1 & 3 & 610.0 & 9 & & & & & & & & & & & 9 & 8 \\
\hline RC. 207.50 & 558.6 & 558.6 & 53 & 390.7 & 3 & 558.2 & 7 & 558.6 & 8 & & & & & & & & & 8 & 21 \\
\hline RC.208.50 & 476.7 & 481.8 & 79 & 316.2 & 4 & 468.5 & 8 & 472.3 & 22 & 7 & 22 & 22 & 10 & 476.7 & 50 & & & 129 & 1,639 \\
\hline R.201.50 & 791.9 & 791.9 & 43 & 788.4 & 3 & 791.7 & 4 & 791.9 & 5 & & & & & & & & & 5 & 4 \\
\hline R.202.50 & 698.5 & 698.5 & 46 & 692.7 & 3 & 698.5 & 8 & & & & & & & & & & & 8 & 9 \\
\hline R.203.50 & 605.3 & 605.9 & 50 & 590.5 & 3 & 598.2 & 6 & 598.5 & 7 & 12 & 8 & 8 & 35 & 605.3 & 12 & & & 62 & 50 \\
\hline R.204.50 & 506.4 & 506.4 & 79 & 474.0 & 5 & 499.8 & 23 & 502.2 & 36 & 326 & 232 & 232 & 35 & 506.4 & 52 & & & 131 & - \\
\hline R.205.50 & 690.1 & 696.7 & 50 & 666.2 & 3 & 681.2 & 5 & 682.3 & 7 & 49 & 28 & 28 & 45 & 690.1 & 12 & & & 62 & 15 \\
\hline R.206.50 & 632.4 & 632.4 & 53 & 609.2 & 3 & 622.8 & 9 & 624.8 & 12 & 63 & 15 & 15 & 85 & 632.4 & 21 & & & 74 & 190 \\
\hline R. 207.50 & 575.5 & 575.5 & 53 & 482.2 & 3 & 561.4 & 11 & 564.1 & 15 & 786 & 136 & o & 70 & 575.5 & 101 & & & 154 & 34,406 \\
\hline R. 208.50 & 487.7 & 487.7 & 96 & 461.4 & 6 & 476.3 & 28 & 481.3 & 94 & 7,385 & 3,622 & 1,282 & 55 & 487.7 & 441 & & & 537 & - \\
\hline R.209.50 & 600.6 & 600.6 & 50 & 582.1 & 3 & 598.5 & 6 & 599.2 & 8 & 1 & 1 & 1 & 5 & 600.6 & 10 & & & 60 & 16 \\
\hline R. 210.50 & 645.6 & 645.6 & 53 & 623.6 & 4 & 633.9 & 8 & 635.7 & 11 & 103 & 34 & 34 & 145 & 645.3 & 40 & 128 & 1 & 94 & 18,545 \\
\hline R.211.50 & 535.5 & 543.3 & 83 & 507.6 & 4 & 526.7 & 8 & 528.3 & 11 & 507 & 443 & 443 & 90 & 535.5 & 96 & & & 179 & 10,543 \\
\hline Avg & & & & 91.1 & & 99.4 & & 99.6 & & & & & & 100.0 & & & & 61 & 2,879 \\
\hline Tot & 27 & & & & & & & & & & & & & & & & & 27 & 23 \\
\hline
\end{tabular}


TABLE 4.2: Detailed results on VRPTW Solomon instances with 100 customers and tight time windows

\begin{tabular}{|c|c|c|c|c|c|c|c|c|c|c|c|c|c|c|c|c|c|c|}
\hline \multirow[b]{2}{*}{ Inst } & \multirow[b]{2}{*}{$z^{*}$} & \multicolumn{2}{|c|}{ Upper Bound } & \multicolumn{2}{|c|}{ Proc. $H^{1}$} & \multicolumn{2}{|c|}{ Proc. $H^{3}$} & \multirow[b]{2}{*}{$\left|\mathscr{F}^{3}\right|$} & \multirow[b]{2}{*}{$\left|\mathscr{B}^{3}\right|$} & \multirow[b]{2}{*}{$\left|\mathscr{R}^{3}\right|$} & \multicolumn{3}{|c|}{ Proc. $H^{4}$} & \multicolumn{2}{|c|}{ Problem $\widetilde{P}$} & \multicolumn{3}{|c|}{$T_{t o t}$} \\
\hline & & $z_{U B}$ & $T$ & $L B_{1}$ & $T$ & $L B_{3}$ & $T$ & & & & $S R 3$ & $L B_{4}$ & $T$ & $|\widetilde{\mathscr{R}}|$ & $T_{c p x}$ & BMR & JPSP & DHL \\
\hline C.101.100 & 827.3 & 827.3 & 96 & 827.3 & 7 & & & & & & & & & & & 7 & 3 & 2 \\
\hline C.102.100 & 827.3 & 827.3 & 106 & 820.3 & 8 & 827.3 & 8 & & & & & & & & & 8 & 12 & 8 \\
\hline C.103.100 & 826.3 & 826.3 & 112 & 809.3 & 9 & 826.3 & 11 & & & & & & & & & 11 & 33 & 28 \\
\hline C.104.100 & 822.9 & 822.9 & 119 & 795.6 & 10 & 822.7 & 31 & 10 & 15 & 10 & & 822.9 & 39 & & 1 & 159 & 4,113 & 86 \\
\hline C.105.100 & 827.3 & 827.3 & 99 & 821.2 & 7 & 827.3 & 8 & & & & & & & & & 8 & 5 & 3 \\
\hline C.106.100 & 827.3 & 827.3 & 102 & 817.7 & 8 & 827.3 & 10 & & & & & & & & & 10 & 7 & 4 \\
\hline C.107.100 & 827.3 & 827.3 & 102 & 818.9 & 7 & 827.3 & 8 & & & & & & & & & 8 & 6 & 4 \\
\hline C.108.100 & 827.3 & 827.3 & 106 & 818.9 & 7 & 827.3 & 8 & & & & & & & & & 8 & 14 & 7 \\
\hline C. 109.100 & 827.3 & 827.3 & 112 & 803.2 & 8 & 827.3 & 10 & & & & & & & & & 10 & 20 & 16 \\
\hline RC.101.100 & $1,619.8$ & $1,619.8$ & 92 & $1,576.2$ & 5 & $1,581.1$ & 5 & 24 & 31 & 298 & 95 & $1,619.8$ & 13 & & & 105 & 12 & 19 \\
\hline RC.102.100 & $1,457.4$ & $1,463.5$ & 99 & $1,398.6$ & 5 & $1,405.5$ & 8 & 428 & 432 & o & 150 & $1,457.4$ & 39 & & & 138 & 76 & 120 \\
\hline RC.103.100 & $1,258.0$ & $1,267.0$ & 102 & $1,214.7$ & 5 & $1,224.5$ & 8 & 811 & 794 & o & 342 & $1,257.6$ & 237 & 118,992 & 96 & 435 & 2,705 & 541 \\
\hline RC.104.100 & $1,132.3$ & $1,132.6$ & 109 & $1,070.3$ & 6 & $1,100.4$ & 35 & 1,610 & 1,371 & o & 255 & $1,129.8$ & 442 & 5,029 & 10 & 561 & 65,806 & 11,773 \\
\hline RC. 105.100 & $1,513.7$ & $1,513.8$ & 99 & $1,467.6$ & 5 & $1,471.7$ & 7 & 81 & 116 & o & 60 & $1,513.7$ & 17 & & & 116 & 26 & 33 \\
\hline RC.106.100 & $1,372.7$ & $1,373.9$ & 96 & $1,301.2$ & 5 & $1,318.5$ & 8 & 293 & 392 & o & 428 & $1,367.2$ & 267 & 40,162 & 50 & 413 & 15,891 & 3,916 \\
\hline RC.107.100 & $1,207.8$ & $1,209.3$ & 99 & $1,156.4$ & 6 & $1,182.7$ & 8 & 136 & 195 & o & 105 & $1,207.8$ & 28 & & & 127 & 153 & 161 \\
\hline RC.108.100 & $1,114.2$ & $1,114.2$ & 102 & $1,049.7$ & 6 & $1,073.0$ & 11 & 1,460 & 1,529 & o & 185 & $1,114.2$ & 213 & & & 315 & 3,365 & 635 \\
\hline R.101.100 & $1,637.7$ & $1,637.7$ & 99 & $1,628.5$ & 5 & $1,630.4$ & 6 & 1 & 1 & 5 & 10 & $1,634.0$ & 9 & 46 & & 108 & 1 & 8 \\
\hline R.102.100 & $1,466.6$ & $1,467.6$ & 109 & $1,462.0$ & 5 & $1,466.2$ & 7 & 1 & 1 & 1 & & $1,466.6$ & 10 & & & 119 & 4 & 3 \\
\hline R.103.100 & $1,208.7$ & $1,208.7$ & 112 & $1,201.7$ & 5 & $1,203.2$ & 6 & 4 & 8 & 24 & 30 & $1,208.7$ & 11 & & & 123 & 23 & 20 \\
\hline R.104.100 & 971.5 & 976.0 & 112 & 947.8 & 6 & 955.7 & 12 & 1,124 & 1,060 & o & 449 & 971.3 & 224 & 119,830 & 7 & 343 & 32,343 & 3,103 \\
\hline R.105.100 & $1,355.3$ & $1,355.3$ & 102 & $1,343.8$ & 5 & $1,345.0$ & 6 & 6 & 8 & 47 & 103 & $1,355.1$ & 11 & 80 & & 113 & 43 & 36 \\
\hline R.106.100 & $1,234.6$ & $1,234.6$ & 109 & $1,224.3$ & 5 & $1,225.7$ & 7 & 24 & 40 & 237 & 160 & $1,234.6$ & 20 & & & 129 & 75 & 87 \\
\hline R.107.100 & $1,064.6$ & $1,064.6$ & 109 & $1,049.9$ & 5 & $1,052.3$ & 7 & 76 & 163 & 703 & 300 & $1,064.3$ & 84 & 193 & & 193 & 1,310 & 416 \\
\hline R.108.100 & 932.1 & 933.7 & 119 & 905.7 & 6 & 913.0 & 10 & 1,882 & 2,066 & o & 280 & 932.1 & 225 & & & 344 & 5,911 & 891 \\
\hline R.109.100 & $1,146.9$ & $1,146.9$ & 102 & $1,129.3$ & 5 & $1,133.5$ & 9 & 30 & 52 & 329 & 233 & $1,144.1$ & 53 & 4,953 & 1 & 156 & 1,432 & 1,127 \\
\hline R.110.100 & $1,068.0$ & $1,075.9$ & 109 & $1,047.8$ & 6 & $1,054.9$ & 9 & 384 & 401 & o & 398 & $1,068.0$ & 143 & 225,584 & 7 & 259 & 1,068 & 426 \\
\hline R.111.100 & $1,048.7$ & $1,048.7$ & 109 & $1,030.5$ & 6 & $1,033.9$ & 10 & 127 & 304 & o & 281 & $1,045.8$ & 106 & 10,620 & 40 & 255 & 83,931 & 5,738 \\
\hline R.112.100 & 948.6 & 948.6 & 116 & 916.6 & 6 & 926.2 & 11 & 2,115 & 4,311 & o & 539 & 946.6 & 721 & 6,214 & 28 & 865 & 202,803 & 16,073 \\
\hline Avg & & & & 97.7 & & 98.7 & & & & & & 99.9 & & & & 188 & 14,524 & 1,562 \\
\hline Tot & 29 & & & & & & & & & & & & & & & 29 & 29 & 29 \\
\hline
\end{tabular}


TABLE 4.3: Detailed results on VRPTW Solomon instances with 100 customers and wide time windows

\begin{tabular}{|c|c|c|c|c|c|c|c|c|c|c|c|c|c|c|c|c|c|c|c|c|}
\hline \multirow[b]{2}{*}{ Inst } & \multirow[b]{2}{*}{$z^{*}$} & \multicolumn{2}{|c|}{ Upper Bound } & \multicolumn{2}{|c|}{ Proc. $H^{1}$} & \multicolumn{2}{|c|}{ Proc. $H^{2}$} & \multicolumn{2}{|c|}{ Proc. $H^{3}$} & \multirow[b]{2}{*}{$\left|\mathscr{F}^{3}\right|$} & \multirow[b]{2}{*}{$\left|\mathscr{B}^{3}\right|$} & \multirow[b]{2}{*}{$\left|\mathscr{R}^{3}\right|$} & \multicolumn{3}{|c|}{ Proc. $H^{4}$} & \multicolumn{2}{|c|}{ Problem $\widetilde{P}$} & \multicolumn{3}{|c|}{$T_{t o t}$} \\
\hline & & $z_{U B}$ & $T$ & $L B_{1}$ & $T$ & $L B_{2}$ & $T$ & $L B_{3}$ & $T$ & & & & $S R 3$ & $L B_{4}$ & $T$ & $|\widetilde{\mathscr{R}}|$ & $T_{c p x}$ & BMR & JPSP & DHL \\
\hline C.201.100 & 589.1 & 589.1 & 228 & 589.1 & 6 & & & & & & & & & & & & & 6 & 203 & 9 \\
\hline C. 202.100 & 589.1 & 589.1 & 244 & 589.1 & 14 & & & & & & & & & & & & & 14 & 3,483 & 49 \\
\hline C. 203.100 & 588.7 & 588.7 & 264 & 588.6 & 14 & 588.7 & 32 & & & & & & & & & & & 32 & 13,070 & 122 \\
\hline C.204.100 & 588.1 & 588.1 & 277 & 577.5 & 15 & 587.5 & 170 & 588.1 & 182 & & & & & & & & & 182 & - & 16,416 \\
\hline C.205.100 & 586.4 & 586.4 & 251 & 586.4 & 12 & & & & & & & & & & & & & 12 & 416 & 15 \\
\hline C.206.100 & 586.0 & 586.0 & 238 & 576.8 & 13 & 586.0 & 28 & & & & & & & & & & & 28 & 594 & 24 \\
\hline C. 207.100 & 585.8 & 585.8 & 244 & 581.8 & 13 & 585.8 & 22 & & & & & & & & & & & 22 & 1,241 & 84 \\
\hline C. 208.100 & 585.8 & 585.8 & 244 & 581.3 & 13 & 585.8 & 22 & & & & & & & & & & & 22 & 555 & 26 \\
\hline RC.201.100 & $1,261.8$ & $1,262.6$ & 139 & $1,240.0$ & 7 & $1,254.4$ & 10 & $1,255.4$ & 12 & 6 & 7 & 34 & 55 & $1,261.7$ & 18 & 585 & & 157 & 229 & 92 \\
\hline RC.202.100 & $1,092.3$ & $1,095.8$ & 152 & $1,003.8$ & 7 & $1,084.7$ & 11 & $1,086.2$ & 13 & 91 & 71 & 655 & 45 & $1,092.3$ & 31 & & & 183 & 312 & 89 \\
\hline RC.203.100 & 923.7 & 923.7 & 185 & 814.3 & 7 & 916.0 & 16 & 919.5 & 22 & 119 & 29 & 269 & 30 & 923.7 & 67 & & & 252 & 14,917 & 324 \\
\hline RC.204.100 & 783.5 & 783.5 & 150 & 687.4 & 20 & 771.1 & 235 & 778.4 & 455 & 1,375 & 339 & o & 50 & 783.5 & 902 & & & 1,052 & - & - \\
\hline RC.205.100 & $1,154.0$ & $1,154.0$ & 149 & $1,054.0$ & 7 & $1,144.0$ & 12 & $1,145.8$ & 14 & 25 & 22 & 117 & 30 & $1,154.0$ & 23 & & & 172 & 221 & 111 \\
\hline RC.206.100 & $1,051.1$ & $1,051.1$ & 172 & 951.5 & 8 & $1,034.4$ & 13 & $1,037.7$ & 16 & 276 & 177 & o & 40 & $1,051.1$ & 55 & & & 227 & 339 & 344 \\
\hline RC.207.100 & 962.9 & 966.6 & 182 & 865.7 & 9 & 940.6 & 25 & 945.8 & 62 & o & o & o & 490 & 962.9 & 26,721 & & & 26,903 & - & 91,405 \\
\hline RC. 208.100 & 776.1 & 777.3 & 215 & 703.9 & 20 & 761.3 & 114 & 765.8 & 168 & 3,909 & 3,306 & o & 110 & 776.1 & 980 & 6,959 & & 1,195 & - & - \\
\hline R.201.100 & $1,143.2$ & $1,148.5$ & 149 & $1,135.2$ & 8 & $1,140.0$ & 15 & $1,140.3$ & 17 & 62 & 44 & o & 100 & $1,143.2$ & 31 & & & 180 & 139 & 78 \\
\hline R.202.100 & $1,029.6$ & $1,036.9$ & 178 & $1,008.6$ & 8 & $1,020.6$ & 19 & $1,021.2$ & 22 & o & o & o & 115 & $1,027.3$ & 1,890 & 575,375 & 1,338 & 3,406 & 8,282 & 1,663 \\
\hline R.203.100 & 870.8 & 872.4 & 198 & 845.7 & 9 & 862.8 & 91 & 865.8 & 165 & o & 564 & o & 100 & 870.8 & 1,941 & & & 2,139 & 54,187 & 641 \\
\hline R.204.100 & 731.3 & 731.3 & 221 & 688.8 & 22 & 721.3 & 64 & 724.2 & 194 & o & o & o & 130 & 731.3 & 216,146 & & & 216,367 & - & - \\
\hline R.205.100 & 949.8 & 949.8 & 191 & 916.0 & 11 & 934.1 & 27 & 938.0 & 31 & 3,779 & 920 & o & 553 & 948.3 & 1,240 & 19,034 & 2 & 1,433 & - & 6,904 \\
\hline R.206.100 & 875.9 & 880.6 & 201 & 834.0 & 13 & 860.2 & 34 & 866.3 & 76 & o & o & o & 145 & 875.9 & 6,474 & & & 6,675 & - & 60,608 \\
\hline R.207.100 & 794.0 & 794.0 & 238 & 746.9 & 16 & 781.6 & 120 & 789.9 & 368 & o & o & o & 35 & 794.0 & 1,163 & & & 1,401 & - & 11,228 \\
\hline R. 208.100 & 701.2 & 701.2 & 284 & 660.9 & 21 & 686.4 & 156 & 690.3 & 2,405 & o & o & o & & m.o. & & & & - & - & - \\
\hline R.209.100 & 854.8 & 855.7 & 136 & 818.7 & 15 & 838.0 & 28 & 840.6 & 59 & o & o & o & 180 & 854.3 & 4,355 & 120,577 & 5 & 4,496 & 78,560 & 22,514 \\
\hline R. 210.100 & 900.5 & 900.8 & 136 & 848.9 & 17 & 883.8 & 30 & 888.2 & 57 & o & o & o & 523 & 900.4 & 39,572 & 53,704 & 3 & 39,711 & - & 400,904 \\
\hline R. 211.100 & 746.7 & 751.7 & 168 & 704.9 & 21 & 729.3 & 133 & 734.1 & 219 & o & o & o & 140 & 746.7 & 10,825 & & & 10,993 & - & - \\
\hline Avg & & & & 95.4 & & 99.0 & & 99.3 & & & & & & 99.9 & & & & 12,202 & 11,047 & 27,893 \\
\hline Tot & 27 & & & & & & & & & & & & & & & & & 26 & 16 & 22 \\
\hline
\end{tabular}

m.o.: GenP4 runs out of memory 
TABLE 4.4: VRPTW Solomon instances: summary

\begin{tabular}{|c|c|c|c|c|c|c|c|c|}
\hline \multirow[b]{2}{*}{ Class } & \multirow[b]{2}{*}{$n$} & \multirow[b]{2}{*}{$N P$} & \multicolumn{3}{|c|}{ Solved } & \multicolumn{3}{|c|}{$T$} \\
\hline & & & BMR & JPSP & DHL & BMR & JPSP & DHL \\
\hline $\mathrm{C} 2$ & 50 & 8 & 8 & 7 & n.a. & 8 & 79 & n.a. \\
\hline $\mathrm{RC} 2$ & 50 & 8 & 8 & 7 & n.a. & 27 & 268 & n.a. \\
\hline $\mathrm{R} 2$ & 50 & 11 & 11 & 9 & n.a. & 124 & 7,086 & n.a. \\
\hline $\mathrm{C} 1$ & 100 & 9 & 9 & 9 & 9 & 25 & 468 & 18 \\
\hline RC1 & 100 & 8 & 8 & 8 & 8 & 276 & 11,004 & 2,150 \\
\hline $\mathrm{R} 1$ & 100 & 12 & 12 & 12 & 12 & 251 & 27,412 & 2,327 \\
\hline $\mathrm{C} 2$ & 100 & 8 & 8 & 7 & 8 & 40 & 2,795 & 2,093 \\
\hline $\mathrm{RC} 2$ & 100 & 8 & 8 & 5 & 6 & 3,767 & 3,204 & 15,394 \\
\hline $\mathrm{R} 2$ & 100 & 11 & 10 & 4 & 8 & 28,680 & 35,292 & 63,068 \\
\hline \multicolumn{6}{|l|}{ Avg } & 3,955 & 9,767 & 12,920 \\
\hline \multicolumn{6}{|c|}{ Solved by JPSP } & 261 & 9,767 & \\
\hline \multicolumn{6}{|c|}{ Solved by DHL } & 1,825 & & 12,920 \\
\hline
\end{tabular}

TABLE 4.5: Optimal solutions of 100-customer VRPTW instances solved by BMR for the first time

\begin{tabular}{|c|c|}
\hline Cost & Route \\
\hline & Solution of RC204. Total cost $=783.5$. Number of vehicles $=4$ \\
\hline 174.6 & 80929562503431292726283032338976638551845691 \\
\hline 139.5 & 815441394244434036353738727193679496 \\
\hline 221.4 & 699812144717161511109871386745997755877252422836590 \\
\hline 248.0 & 66642049191848212357529982536078737978464553144628855100706168 \\
\hline & Solution of RC208. Total cost $=776.1$. Number of vehicles $=4$ \\
\hline 132.5 & 6142443938363537404341727193965481 \\
\hline 218.7 & $90 \quad 6582995283 \quad 6449191848212325 \quad 77587597 \quad 5987 \quad 7486 \quad 572422 \quad 2066$ \\
\hline 226.6 & 69988826779737812144717161513911105360846445531701005568 \\
\hline 198.3 & 949295676250343129272628303233768963855184569180 \\
\hline & Solution of R204. Total cost $=731.3$. Number of vehicles $=5$. \\
\hline 8.8 & 53 \\
\hline 211.6 & $27691507637933819512066 \quad 65713534782924552554806877 \quad 12 \quad 2628$ \\
\hline 150.5 & 257424315412275562367394727473214058 \\
\hline 136.2 & 69495929885914414388616619399965997873710013 \\
\hline 224.2 & 5231887824819116210703032906364493647468451784560831889 \\
\hline 173.1 & $\begin{array}{l}\text { Solution of R211. Total cost }=746.7 \text {. Number of vehicles }=4 \text {. } \\
5340217341227523673925552429688054456747226\end{array}$ \\
\hline 214.0 & 27693188782464736496463903266716535347879377501 \\
\hline 194.4 & 5218835999592988561168638441442431557287973710091935996941358 \\
\hline 165.2 & 28127633819512030701062111948845178460689 \\
\hline
\end{tabular}


TABLE 4.6: VRPTW: impact of parameter $\Delta\left(N_{i}\right)$ in procedure $H^{2}$

\begin{tabular}{|c|c|c|c|c|c|c|c|c|c|c|c|c|c|c|c|}
\hline \multirow[b]{3}{*}{ Inst } & \multirow[b]{3}{*}{$\mathrm{z}^{*}$} & \multicolumn{2}{|l|}{$H^{1}$} & \multicolumn{8}{|c|}{$H^{2}$} & \multicolumn{2}{|l|}{$H^{3}$} & \multicolumn{2}{|c|}{$H^{4}$} \\
\hline & & \multirow[b]{2}{*}{$L B_{1}$} & \multirow[b]{2}{*}{$T$} & \multicolumn{2}{|c|}{$\Delta\left(N_{i}\right)=5$} & \multicolumn{2}{|c|}{$\Delta\left(N_{i}\right)=8$} & \multicolumn{2}{|c|}{$\Delta\left(N_{i}\right)=10$} & \multicolumn{2}{|c|}{$\Delta\left(N_{i}\right)=12$} & \multirow[b]{2}{*}{$L B_{3}$} & \multirow[b]{2}{*}{$T$} & \multirow[b]{2}{*}{$L B_{4}$} & \multirow[b]{2}{*}{$T$} \\
\hline & & & & $L B_{2}$ & $T$ & $L B_{2}$ & $T$ & $L B_{2}$ & $T$ & $L B_{2}$ & $T$ & & & & \\
\hline R.207.50 & 575.5 & 482.2 & 3 & 558.5 & 7 & 561.4 & 11 & 561.6 & 13 & 562.4 & 16 & 564.1 & 15 & 575.5 & 101 \\
\hline R.208.50 & 487.7 & 461.4 & 6 & 475.1 & 22 & 476.3 & 28 & 476.6 & 37 & 477.3 & 57 & 481.3 & 94 & 487.7 & 441 \\
\hline R.108.100 & 932.1 & 905.7 & 6 & 909.8 & 7 & 910.3 & 10 & 910.3 & 14 & 910.8 & 26 & 913.0 & 10 & 932.1 & 225 \\
\hline R.112.100 & 948.6 & 916.6 & 6 & 924.2 & 7 & 925.0 & 10 & 925.0 & 18 & 925.0 & 39 & 926.2 & 11 & 946.6 & 721 \\
\hline RC.203.100 & 923.7 & 814.3 & 7 & 914.6 & 11 & 916.0 & 16 & 916.5 & 29 & 917.4 & 43 & 919.5 & 22 & 923.7 & 67 \\
\hline RC.204.100 & 783.5 & 687.4 & 20 & 753.9 & 91 & 771.1 & 235 & 772.9 & 638 & 773.0 & 976 & 778.4 & 455 & 783.5 & 902 \\
\hline RC.206.100 & $1,051.1$ & 951.5 & 8 & $1,022.8$ & 13 & $1,034.4$ & 13 & $1,034.9$ & 15 & $1,035.4$ & 16 & $1,037.7$ & 16 & $1,051.1$ & 55 \\
\hline RC.207.100 & 962.9 & 865.7 & 9 & 918.4 & 23 & 940.6 & 25 & 940.9 & 38 & 941.6 & 48 & 945.8 & 62 & 962.9 & 26,721 \\
\hline RC.208.100 & 776.1 & 703.9 & 20 & 740.9 & 42 & 761.3 & 114 & 762.6 & 223 & 763.8 & 352 & 765.8 & 168 & 776.1 & 980 \\
\hline R.202.100 & $1,029.6$ & $1,008.6$ & 8 & $1,018.6$ & 9 & $1,020.6$ & 19 & $1,020.6$ & 23 & $1,020.7$ & 24 & $1,021.2$ & 22 & $1,027.3$ & 1,890 \\
\hline R.203.100 & 870.8 & 845.7 & 9 & 858.0 & 64 & 862.8 & 91 & 864.3 & 108 & 865.0 & 149 & 865.8 & 165 & 870.8 & 1,941 \\
\hline R.204.100 & 731.3 & 688.8 & 22 & 719.1 & 54 & 721.3 & 64 & 722.2 & 180 & 722.2 & 298 & 724.2 & 194 & 731.3 & 216,146 \\
\hline R.205.100 & 949.8 & 916.0 & 11 & 928.0 & 14 & 934.1 & 27 & 934.6 & 31 & 935.0 & 32 & 938.0 & 31 & 948.3 & 1,240 \\
\hline R.206.100 & 875.9 & 834.0 & 13 & 857.6 & 21 & 860.2 & 34 & 860.7 & 36 & 861.6 & 53 & 866.3 & 76 & 875.9 & 6,474 \\
\hline R.207.100 & 794.0 & 746.9 & 16 & 780.4 & 83 & 781.6 & 120 & 781.6 & 191 & 782.9 & 236 & 789.9 & 368 & 794.0 & 1,163 \\
\hline R.209.100 & 854.8 & 818.7 & 15 & 833.8 & 16 & 837.0 & 28 & 837.6 & 30 & 838.8 & 34 & 840.6 & 59 & 854.3 & 4,355 \\
\hline R.210.100 & 900.5 & 848.9 & 17 & 880.6 & 18 & 883.8 & 30 & 884.8 & 37 & 884.8 & 49 & 888.2 & 57 & 900.4 & 39,572 \\
\hline R.211.100 & 746.7 & 704.9 & 21 & 727.1 & 102 & 729.3 & 133 & 731.3 & 189 & 731.3 & 417 & 734.1 & 219 & 746.7 & 10,825 \\
\hline Avg & & 93.3 & & 97.5 & & 98.2 & & 98.3 & & 98.4 & & 98.7 & & 99.9 & \\
\hline
\end{tabular}


TABLE 4.7: VRPTW: effectiveness of the Dominance and Fathoming Rules applied in GenP4

\begin{tabular}{|c|c|c|c|c|c|c|c|c|c|c|}
\hline \multirow[b]{2}{*}{ Inst } & \multicolumn{5}{|c|}{ Generating $\mathscr{F}$} & \multicolumn{5}{|c|}{ Generating $\mathscr{B}$} \\
\hline & States & $\%$ Dom 1 & \%Fath1 & $\%$ Fath2 & $|\mathscr{F}|$ & States & $\%$ Dom1 & \%Fath1 & \%Fath2 & $|\mathscr{B}|$ \\
\hline RC.207.100 & 41 & 38.4 & 48.7 & 1.8 & 5 & 21 & 37.9 & 49.2 & 1.7 & 2 \\
\hline R.202.100 & 11 & 49.0 & 41.4 & 1.6 & 1 & 7 & 49.2 & 39.7 & 1.1 & 1 \\
\hline R.203.100 & 32 & 46.9 & 42.3 & 1.5 & 3 & & & & & \\
\hline R.204.100 & 180 & 43.8 & 43.6 & 0.4 & 22 & 283 & 37.1 & 48.3 & 0.4 & 40 \\
\hline R.206.100 & 235 & 58.8 & 32.1 & 1.2 & 19 & 179 & 67.4 & 24.2 & 1.3 & 13 \\
\hline R.207.100 & 81 & 54.4 & 38.6 & 1.0 & 5 & 43 & 54.8 & 36.4 & 0.9 & 3 \\
\hline R.209.100 & 102 & 55.2 & 37.9 & 1.3 & 6 & 31 & 56.1 & 38.0 & 0.8 & 2 \\
\hline R.210.100 & 184 & 57.5 & 33.7 & 0.9 & 15 & 222 & 59.7 & 31.4 & 1.0 & 18 \\
\hline R.211.100 & 496 & 68.6 & 24.1 & 0.6 & 33 & 371 & 56.7 & 34.4 & 0.3 & 32 \\
\hline Avg & & 52.5 & 38.0 & 1.1 & & & 52.4 & 37.7 & 0.9 & \\
\hline
\end{tabular}

TABLE 4.8: VRPTW: effectiveness of the Dominance and Fathoming Rules applied in GenPF

\begin{tabular}{|c|c|c|c|c|c|c|c|c|}
\hline \multirow[b]{2}{*}{ Inst } & \multicolumn{4}{|c|}{ Generating $\mathscr{F}$} & \multicolumn{4}{|c|}{ Generating $\mathscr{B}$} \\
\hline & States & \%Fath5 & \%Fath4 & $|\mathscr{F}|$ & States & $\%$ Fath5 & \%Fath4 & $|\mathscr{B}|$ \\
\hline R.202.100 & 10 & 37.1 & 51.8 & 1 & 3 & 47.1 & 46.9 & 1 \\
\hline R.209.100 & 339 & 50.0 & 42.2 & 26 & 113 & 51.3 & 43.4 & 6 \\
\hline R.210.100 & 108 & 45.8 & 46.0 & 9 & 210 & 55.1 & 35.4 & 20 \\
\hline Avg & & 44.3 & 46.7 & & & 51.2 & 41.9 & \\
\hline
\end{tabular}


TABLE 4.9: Results on CVRP instances of class A

\begin{tabular}{|c|c|c|c|c|c|c|c|c|c|c|c|c|c|c|c|c|c|c|}
\hline \multirow[b]{2}{*}{ Inst } & \multirow[b]{2}{*}{$z_{U B}$} & \multirow[b]{2}{*}{$z^{*}$} & \multicolumn{2}{|c|}{ Proc. $H^{1}$} & \multicolumn{2}{|c|}{ Proc. $H^{3}$} & \multirow[b]{2}{*}{$\left|\mathscr{F}^{3}\right|$} & \multirow[b]{2}{*}{$\left|\mathscr{R}^{3}\right|$} & \multicolumn{4}{|c|}{ Proc. $H^{4}$} & \multicolumn{2}{|c|}{ Problem $\widetilde{P}$} & \multicolumn{4}{|c|}{$T_{t o t}$} \\
\hline & & & $L B_{1}$ & $T$ & $L B_{3}$ & $T$ & & & $S R 3$ & $L B_{4}$ & $U B_{4}$ & $T$ & $|\widetilde{\mathscr{R}}|$ & $T_{c p x}$ & BMR & $\mathrm{BCM}$ & FLL $(s)$ & LLE \\
\hline A-n37-k5 & 669 & 669 & 664.7 & 3 & 666.1 & 5 & 5 & 1 & 3 & 669.0 & & 6 & & & 6 & 13 & $19(-)$ & 25 \\
\hline A-n37-k6 & 949 & 949 & 929.8 & 3 & 937.9 & 7 & 9 & 10 & 37 & 941.6 & & 8 & 1,968 & $<0.1$ & 8 & 12 & 379 (3) & 531 \\
\hline A-n38-k5 & 730 & 730 & 717.9 & 4 & 722.4 & 7 & 10 & 10 & 41 & 728.7 & & 7 & 267 & $<0.1$ & 7 & 18 & $26(-)$ & 116 \\
\hline A-n39-k5 & 822 & 822 & 815.1 & 4 & 818.7 & 7 & 8 & 2 & 14 & 822.0 & & 7 & & & 7 & 19 & $167(3)$ & 138 \\
\hline A-n39-k6 & 831 & 831 & 820.5 & 4 & 823.7 & 6 & 5 & 4 & 31 & 831.0 & & 7 & 149 & $<0.1$ & 7 & 12 & $98(3)$ & 109 \\
\hline A-n44-k6 & 937 & 937 & 932.4 & 2 & 935.3 & 5 & 2 & 1 & 2 & 937.0 & & 5 & 124 & $<0.1$ & 5 & 102 & $90(2)$ & 620 \\
\hline A-n45-k6 & 944 & 944 & 933.0 & 6 & 941.1 & 11 & 4 & 1 & 4 & 944.0 & & 12 & 114 & $<0.1$ & 12 & 77 & $170(3)$ & 157 \\
\hline A-n 45-k7 & 1,146 & 1,146 & $1,135.7$ & 5 & $1,141.4$ & 10 & 5 & 4 & 62 & $1,146.0$ & & 11 & 316 & $<0.1$ & 11 & 35 & $331(3)$ & 19,414 \\
\hline A-n46-k7 & 914 & 914 & 912.6 & 6 & 914.0 & 8 & 1 & 1 & 0 & 914.0 & & 8 & & & 8 & 11 & $92(2)$ & 50 \\
\hline A-n48-k7 & 1,073 & 1,073 & $1,065.9$ & 9 & $1,071.8$ & 14 & 2 & 1 & 1 & $1,073.0$ & & 15 & 129 & $<0.1$ & 15 & 20 & $166(3)$ & 372 \\
\hline A-n53-k7 & 1,010 & 1,010 & $1,001.1$ & 11 & $1,003.8$ & 21 & 15 & 12 & 78 & $1,009.7$ & & 24 & 364 & $<0.1$ & 24 & 28 & $611(3)$ & 363 \\
\hline A-n54-k7 & 1,167 & 1,167 & $1,148.7$ & 12 & $1,156.6$ & 25 & 84 & 207 & 91 & $1,166.1$ & & 35 & 709 & $<0.1$ & 35 & 86 & $1,409(3)$ & 7,246 \\
\hline A-n55-k9 & 1,073 & 1,073 & $1,064.2$ & 9 & $1,067.8$ & 18 & 10 & 5 & 30 & $1,070.7$ & & 19 & 771 & $<0.1$ & 19 & 19 & $84(3)$ & 468 \\
\hline A-n60-k9 & 1,354 & 1,354 & $1,339.0$ & 17 & $1,343.9$ & 32 & 78 & 201 & 108 & $1,351.7$ & & 44 & 2,704 & 2 & 46 & 111 & 3,080 (3) & - \\
\hline A-n61-k9 & 1,034 & 1,034 & $1,016.0$ & 13 & $1,023.2$ & 31 & 45 & 45 & 80 & $1,032.0$ & & 37 & 1,009 & $<0.1$ & 37 & 34 & $1,883(3)$ & 68,636 \\
\hline A-n62-k8 & 1,290 & 1,288 & $1,271.8$ & 19 & $1,280.5$ & 42 & 229 & 468 & 106 & $1,285.4$ & & 65 & 13,099 & 31 & 96 & 1,342 & $3,102(3)$ & - \\
\hline A-n63-k9 & 1,616 & 1,616 & $1,596.8$ & 18 & $1,608.6$ & 37 & 20 & 17 & 32 & $1,616.0$ & & 40 & 303 & $<0.1$ & 40 & 44 & $1,046(3)$ & - \\
\hline A-n63-k10 & 1,315 & 1,314 & $1,291.9$ & 20 & $1,302.4$ & 42 & 58 & 80 & 60 & $1,309.5$ & & 47 & 4,616 & 4 & 51 & 129 & 4,988 (3) & - \\
\hline A-n64-k9 & 1,402 & 1,401 & $1,376.7$ & 16 & $1,387.5$ & 36 & 146 & 271 & 84 & $1,395.3$ & & 49 & 7,810 & 14 & 63 & 120 & $11,254(3)$ & - \\
\hline A-n65-k9 & 1,174 & 1,174 & $1,161.0$ & 14 & $1,165.6$ & 29 & 20 & 24 & 29 & $1,174.0$ & & 32 & 294 & $<0.1$ & 32 & 38 & $516(3)$ & 1,324 \\
\hline A-n69-k9 & 1,159 & 1,159 & $1,136.7$ & 20 & $1,143.9$ & 43 & 131 & 363 & 84 & $1,156.8$ & & 55 & 1,659 & $<0.1$ & 55 & 124 & $7,171(3)$ & - \\
\hline A-n80-k10 & 1,763 & 1,763 & $1,746.2$ & 30 & $1,755.5$ & 63 & 126 & 80 & 89 & $1,760.7$ & & 78 & 2,513 & 4 & 82 & 194 & $6,464(3)$ & - \\
\hline Avg & & & 98.8 & & 99.4 & & & & & 99.9 & & & & & 30 & 118 & 1,961 & 6,638 \\
\hline Solved & & 22 & & & & & & & & & & & & & 22 & 22 & 22 & 15 \\
\hline
\end{tabular}


TABLE 4.10: Results on CVRP instances of class B

\begin{tabular}{|c|c|c|c|c|c|c|c|c|c|c|c|c|c|c|c|c|c|c|}
\hline \multirow[b]{2}{*}{ Inst } & \multirow[b]{2}{*}{$z_{U B}$} & \multirow[b]{2}{*}{$z^{*}$} & \multicolumn{2}{|c|}{ Proc. $H^{1}$} & \multicolumn{2}{|c|}{ Proc. $H^{3}$} & \multirow[b]{2}{*}{$\left|\mathscr{F}^{3}\right|$} & \multirow[b]{2}{*}{$\left|\mathscr{R}^{3}\right|$} & \multicolumn{4}{|c|}{ Proc. $H^{4}$} & \multicolumn{2}{|c|}{ Problem $\widetilde{P}$} & \multicolumn{4}{|c|}{$T_{t o t}$} \\
\hline & & & $L B_{1}$ & $T$ & $L B_{3}$ & $T$ & & & $S R 3$ & $L B_{4}$ & $U B_{4}$ & $T$ & $|\widetilde{\mathscr{R}}|$ & $T_{c p x}$ & BMR & $\mathrm{BCM}$ & FLL $(s)$ & LLE \\
\hline B-n38-k6 & 805 & 805 & 801.5 & 3 & 803.8 & 4 & 2 & 1 & 4 & 805.0 & & 4 & & & 4 & 16 & $14(-)$ & 37 \\
\hline B-n39-k5 & 549 & 549 & 549.0 & 5 & 549.0 & 6 & 1 & 1 & - & 549.0 & & 6 & & & 6 & 7 & $3(-)$ & 9 \\
\hline B-n41-k6 & 829 & 829 & 827.7 & 3 & 828.7 & 4 & 1 & 1 & 2 & 829.0 & & 4 & & & 4 & 15 & $18(-)$ & 42 \\
\hline B-n43-k6 & 742 & 742 & 735.7 & 4 & 736.8 & 5 & 88 & 29 & 91 & 740.0 & & 20 & 9,591 & 3 & 23 & 37 & $29(-)$ & 125 \\
\hline B-n44-k7 & 909 & 909 & 909.0 & 2 & 909.0 & 3 & 1 & 1 & - & 909.0 & & 3 & & & 3 & 24 & $9(-)$ & 8 \\
\hline B-n45-k5 & 751 & 751 & 748.8 & 6 & 750.9 & 8 & 2 & 1 & - & 751.0 & & 9 & & & 9 & 16 & $16(-)$ & 46 \\
\hline B-n45-k6 & 678 & 678 & 675.3 & 5 & 677.0 & 8 & 11 & 1 & - & 678.0 & & 10 & & & 10 & 61 & $279(3)$ & 299 \\
\hline B-n50-k7 & 741 & 741 & 741.0 & 3 & 741.0 & 4 & 1 & 1 & - & 741.0 & & 4 & & & 4 & 24 & $6(-)$ & 11 \\
\hline B-n50-k8 & 1,312 & 1,312 & $1,291.1$ & 11 & $1,302.6$ & 24 & 1,011 & 334 & 92 & $1,308.8$ & 1,312 & 118 & 23,073 & 29 & 147 & 662 & $2,845(3)$ & 31,026 \\
\hline B-n51-k7 & 1,032 & 1,032 & $1,025.8$ & 8 & $1,026.7$ & 11 & 339 & 27 & 71 & $1,032.0$ & & 32 & 8,793 & 2 & 34 & 52 & $46(-)$ & 209 \\
\hline B-n52-k7 & 747 & 747 & 746.1 & 7 & 746.1 & 7 & 2 & 1 & 3 & 747.0 & & 8 & & & 8 & 29 & $9(-)$ & 25 \\
\hline B-n56-k7 & 707 & 707 & 704.3 & 7 & 704.8 & 9 & 28 & 1 & - & 705.0 & & 11 & 1,653 & ¡0.1 & 11 & 73 & $22(-)$ & 46 \\
\hline B-n57-k7 & 1,153 & 1,153 & $1,149.9$ & 16 & $1,152.8$ & 22 & 6 & 1 & - & $1,153.0$ & & 25 & & & 25 & 184 & $168(-)$ & 441 \\
\hline B-n57-k9 & 1,598 & 1,598 & $1,593.7$ & 11 & $1,596.0$ & 15 & 9 & 1 & 30 & $1,598.0$ & & 19 & & & 19 & 60 & $193(3)$ & 1,366 \\
\hline B-n63-k10 & 1,496 & 1,496 & $1,483.8$ & 15 & $1,486.5$ & 25 & 472 & 278 & 31 & $1,496.0$ & & 52 & 10,360 & 3 & 55 & 94 & $682(-)$ & 6,513 \\
\hline B-n64-k9 & 861 & 861 & 859.7 & 11 & 860.2 & 13 & 17 & 1 & 3 & 861.0 & & 15 & & & 15 & 70 & $86(-)$ & 42 \\
\hline B-n66-k9 & 1,316 & 1,316 & $1,301.7$ & 17 & $1,307.7$ & 27 & 2,547 & 1,331 & 137 & $1,315.2$ & 1,330 & 283 & 13,442 & 9 & 292 & 227 & 1,778 & 24,424 \\
\hline B-n67-k10 & 1,032 & 1,032 & $1,025.8$ & 12 & $1,027.4$ & 18 & 67 & 14 & 85 & $1,032.0$ & & 41 & 9,400 & 2 & 43 & 287 & $568(-)$ & 3,309 \\
\hline B-n68-k9 & 1,275 & 1,272 & $1,261.1$ & 23 & $1,263.2$ & 34 & 2,267 & 1,103 & 141 & $1,267.6$ & 1,272 & 336 & 55,097 & 190 & 526 & 6,168 & $87,436(3)$ & \\
\hline B-n78-k10 & 1,221 & 1,221 & $1,208.4$ & 23 & $1,214.9$ & 36 & 266 & 71 & 52 & $1,221.0$ & & 91 & 11,131 & 6 & 97 & 229 & $1,053(3)$ & 87,408 \\
\hline Avg & & & 99.5 & & 99.7 & & & & & 99.9 & & & & & 67 & 417 & 4,763 & 8,178 \\
\hline Solved & & 20 & & & & & & & & & & & & & 20 & 20 & 20 & 19 \\
\hline
\end{tabular}


TABLE 4.11: Results on CVRP instances of classes E and M

\begin{tabular}{|c|c|c|c|c|c|c|c|c|c|c|c|c|c|c|c|c|c|c|}
\hline \multirow[b]{2}{*}{ Inst } & \multirow[b]{2}{*}{$z_{U B}$} & \multirow[b]{2}{*}{$z^{*}$} & \multicolumn{2}{|c|}{ Proc. $H^{1}$} & \multicolumn{2}{|c|}{ Proc. $H^{3}$} & \multirow[b]{2}{*}{$\left|\mathscr{F}^{3}\right|$} & \multirow[b]{2}{*}{$\left|\mathscr{R}^{3}\right|$} & \multicolumn{4}{|c|}{ Proc. $H^{4}$} & \multicolumn{2}{|c|}{ Problem $\widetilde{P}$} & \multicolumn{4}{|c|}{$T_{t o t}$} \\
\hline & & & $L B_{1}$ & $T$ & $L B_{3}$ & $T$ & & & $S R 3$ & $L B_{4}$ & $U B_{4}$ & $T$ & $|\widetilde{\mathscr{R}}|$ & $T_{c p x}$ & BMR & $\mathrm{BCM}$ & FLL $(s)$ & LLE \\
\hline E-n51-k5 & 521 & 521 & 516.9 & 3 & 517.6 & 7 & 7 & 7 & 14 & 521.0 & & 8 & & & 8 & 12 & $65(-)$ & 59 \\
\hline E-n76-k8 & 735 & 735 & 724.3 & 13 & 725.1 & 45 & 218 & 1,249 & 160 & 734.0 & & 81 & 1,765 & 1 & 82 & 873 & $22,891(2)$ & - \\
\hline E-n76-k10 & 830 & 830 & 815.4 & 18 & 816.5 & 54 & 317 & 1,476 & 143 & 826.2 & & 81 & 10,696 & 33 & 114 & 174 & $80,722(3)$ & - \\
\hline E-n76-k14 & 1,021 & 1,021 & $1,004.3$ & 15 & $1,007.0$ & 36 & 109 & 255 & 93 & $1,014.7$ & & 42 & 8,965 & 10 & 52 & 44 & $48,637(3)$ & - \\
\hline E-n101-k8 & 815 & 815 & 801.8 & 30 & 808.8 & 218 & 1,584 & o & 118 & 815.0 & 818 & 579 & & & 579 & - & $801,963(3)$ & - \\
\hline M-n101-k10 & 820 & 820 & 819.9 & 18 & 820.0 & 34 & 50 & 1 & - & 820.0 & & 35 & & & 35 & 47 & $119(-)$ & 33 \\
\hline M-n121-k7 & 1,034 & 1,034 & $1,028.5$ & 94 & $1,032.5$ & 611 & 2,961 & 12 & 36 & $1,033.4$ & 1,035 & 1,247 & 12,440 & 2 & 1,249 & 2,448 & $25,678(3)$ & - \\
\hline M-n151-k12 & 1,015 & & 993.4 & 131 & $1,004.3$ & 380 & o & o & & m.o. & & & & & - & - & - & - \\
\hline M-n200-k16 & - & & $1,240.3$ & 109 & $1,256.6$ & 319 & o & o & & m.o. & & & & & - & - & - & - \\
\hline M-n200-k17 & 1,275 & & $1,243.1$ & 139 & $1,258.7$ & 436 & o & o & & m.o. & & & & & - & - & - & - \\
\hline Avg & & & 98.5 & & 99.0 & & & & & 99.8 & & & & & 303 & 1,025 & 126,987 & 39,592 \\
\hline
\end{tabular}

TABLE 4.12: Results on CVRP instances of class F

\begin{tabular}{|c|c|c|c|c|c|c|c|c|c|c|c|c|c|c|c|c|c|c|}
\hline \multirow[b]{2}{*}{ Inst } & \multirow[b]{2}{*}{$z_{U B}$} & \multirow[b]{2}{*}{$z^{*}$} & \multicolumn{2}{|c|}{ Proc. $H^{1}$} & \multicolumn{2}{|c|}{ Proc. $H^{3}$} & \multirow[b]{2}{*}{$\left|\mathscr{F}^{3}\right|$} & \multirow[b]{2}{*}{$\left|\mathscr{R}^{3}\right|$} & \multicolumn{4}{|c|}{ Proc. $H^{4}$} & \multicolumn{2}{|c|}{ Problem $\widetilde{P}$} & \multicolumn{4}{|c|}{$T_{t o t}$} \\
\hline & & & $L B_{1}$ & $T$ & $L B_{3}$ & $T$ & & & $S R 3$ & $L B_{4}$ & $U B_{4}$ & $T$ & $|\widetilde{\mathscr{R}}|$ & $T_{c p x}$ & BMR & $\mathrm{BCM}$ & FLL $(s)$ & LLE \\
\hline F-n $45-\mathrm{k} 4$ & 724 & 724 & 723.9 & 20 & 724.0 & 23 & 4 & 1 & 0 & 724.0 & & 23 & & & 23 & & $8(-)$ & 6 \\
\hline F-n72-k4 & 237 & 237 & 233.0 & 249 & 236.2 & 301 & 228 & 10 & 1 & 237.0 & & 304 & & & 304 & & $121(-)$ & 40 \\
\hline F-n135-k7 & 1,162 & 1,162 & $1,158.0$ & 1,057 & m.o. & & & & & & & & & & - & & $7,065(-)$ & 3,092 \\
\hline Avg & & & 99.3 & & 99.8 & & & & & 100.0 & & & & & 164 & & 2,398 & 1,046 \\
\hline Solved & & 3 & & & & & & & & & & & & & 2 & & 3 & 3 \\
\hline
\end{tabular}

m.o.: GenP runs out of memory 
TABLE 4.13: Results on CVRP instances of class P

\begin{tabular}{|c|c|c|c|c|c|c|c|c|c|c|c|c|c|c|c|c|c|c|}
\hline \multirow[b]{2}{*}{ Inst } & \multirow[b]{2}{*}{$z_{U B}$} & \multirow[b]{2}{*}{$z^{*}$} & \multicolumn{2}{|c|}{ Proc. $H^{1}$} & \multicolumn{2}{|c|}{ Proc. $H^{3}$} & \multirow[b]{2}{*}{$\left|\mathscr{F}^{3}\right|$} & \multirow[b]{2}{*}{$\left|\mathscr{R}^{3}\right|$} & \multicolumn{4}{|c|}{ Proc. $H^{4}$} & \multicolumn{2}{|c|}{ Problem $\widetilde{P}$} & \multicolumn{4}{|c|}{$T_{t o t}$} \\
\hline & & & $L B_{1}$ & $T$ & $L B_{3}$ & $T$ & & & $S R 3$ & $L B_{4}$ & $U B_{4}$ & $T$ & $|\widetilde{\mathscr{R}}|$ & $T_{c p x}$ & BMR & $\mathrm{BCM}$ & FLL $(s)$ & LLE \\
\hline P-n16-k8 & 450 & 450 & 448.0 & $<0.1$ & 448.0 & 1 & 1 & 1 & 1 & 450.0 & & 1 & & & 1 & 1 & $1(2)$ & 10 \\
\hline P-n19-k2 & 212 & 212 & 210.7 & $<0.1$ & 212.0 & 1 & 1 & 1 & - & 212.0 & & 1 & & & 1 & 1 & $1(-)$ & 5 \\
\hline P-n20-k2 & 216 & 216 & 212.8 & $<0.1$ & 215.6 & 1 & 1 & 1 & 2 & 216.0 & & 1 & & & 1 & 2 & $1(-)$ & 15 \\
\hline P-n21-k2 & 211 & 211 & 211.0 & $<0.1$ & 211.0 & 1 & 1 & 1 & - & 211.0 & & 1 & & & 1 & 1 & $1(-)$ & 3 \\
\hline P-n22-k2 & 216 & 216 & 214.8 & 1 & 215.4 & 1 & 1 & 1 & 1 & 216.0 & & 1 & & & 1 & 3 & $2(-)$ & 16 \\
\hline P-n22-k8 & 603 & 603 & 602.9 & $<0.1$ & 603.0 & 1 & 1 & 1 & - & 603.0 & & 1 & & & 1 & 12 & $3(2)$ & 44 \\
\hline P-n23-k8 & 529 & 529 & 529.0 & $<0.1$ & 529.0 & 1 & 1 & 1 & - & 529.0 & & 1 & & & 1 & 1 & $18(2)$ & 69 \\
\hline P-n40-k5 & 458 & 458 & 456.1 & 1 & 456.6 & 2 & 1 & 1 & 11 & 458.0 & & 2 & & & 2 & 26 & $34(-)$ & 19 \\
\hline P-n45-k5 & 510 & 510 & 504.2 & 3 & 504.8 & 6 & 1 & 1 & 19 & 510.0 & & 6 & & & 6 & 21 & $194(3)$ & 76 \\
\hline P-n50-k7 & 554 & 554 & 548.7 & 4 & 549.7 & 8 & 6 & 5 & 6 & 554.0 & & 9 & & & 9 & 16 & $143(3)$ & 805 \\
\hline P-n50-k8 & 649 & 631 & 613.4 & 6 & 615.9 & 15 & 47 & 70 & 113 & 625.1 & 631 & 64 & 2,993 & 4 & 68 & 596 & $9,272(3)$ & - \\
\hline P-n50-k10 & 696 & 696 & 685.4 & 4 & 689.2 & 8 & 6 & 6 & 45 & 694.9 & & 8 & 393 & 1 & 9 & 10 & $304(3)$ & 73,016 \\
\hline P-n51-k10 & 741 & 741 & 730.0 & 4 & 734.0 & 10 & 6 & 5 & 49 & 740.9 & & 10 & 202 & 1 & 11 & 9 & $105(3)$ & 82,469 \\
\hline P-n55-k7 & 568 & 568 & 556.5 & 7 & 559.1 & 12 & 50 & 121 & 97 & 565.7 & & 16 & 2,410 & 1 & 17 & 205 & $4,649(2)$ & 11,178 \\
\hline P-n55-k8 & 588 & 588 & 577.3 & 6 & 579.3 & 13 & 39 & 98 & 103 & 584.7 & & 16 & 3,851 & 2 & 18 & 74 & $1,822(2)$ & \\
\hline P-n55-k10 & 699 & 694 & 677.8 & 5 & 680.6 & 15 & 112 & 314 & 84 & 689.1 & & 19 & 18,061 & 10 & 29 & 66 & $9,076(3)$ & - \\
\hline P-n55-k15 & 993 & 989 & 966.6 & 7 & 970.7 & 25 & 20 & 18 & 46 & 984.5 & & 26 & 1,501 & 1 & 27 & 8 & $1,944(3)$ & - \\
\hline P-n60-k10 & 756 & 744 & 736.8 & 9 & 739.3 & 22 & 125 & 395 & 57 & 743.1 & & 28 & 53,850 & 2 & 30 & 59 & $570(3)$ & - \\
\hline P-n60-k15 & 1,033 & 968 & 959.8 & 6 & 963.2 & 15 & 3 & 2 & 45 & 967.8 & 968 & 73 & 241 & $<0.1$ & 73 & 8 & $442(3)$ & - \\
\hline P-n65-k10 & 792 & 792 & 784.5 & 5 & 787.9 & 13 & 6 & 6 & 27 & 792.0 & & 14 & & & 14 & 16 & $422(3)$ & - \\
\hline P-n70-k10 & 834 & 827 & 811.5 & 13 & 813.6 & 34 & 147 & 483 & 129 & 823.0 & 827 & 150 & 7,925 & 16 & 166 & 774 & $24,039(3)$ & - \\
\hline P-n76-k4 & 593 & 593 & 587.0 & 13 & 590.0 & 89 & 174 & 569 & 65 & 593.0 & & 118 & & & 118 & 2,211 & $572(-)$ & 535 \\
\hline P-n76-k5 & 627 & 627 & 614.1 & 5 & 619.5 & 87 & 905 & 3,707 & 137 & 627.0 & & 282 & 401 & $<0.1$ & 282 & - & $14,546(-)$ & 10,970 \\
\hline P-n101-k4 & 681 & 681 & 668.5 & 13 & 678.7 & 73 & 4,475 & 8,811 & 106 & 681.0 & 681 & 1,154 & 458 & 1 & 1,155 & - & $1,253(-)$ & 281 \\
\hline Avg & & & 98.8 & & 99.2 & & & & & 99.8 & & & & & 85 & 187 & 2,892 & 11,219 \\
\hline Solved & & 24 & & & & & & & & & & & & & 24 & 22 & 24 & 16 \\
\hline
\end{tabular}


TABLE 4.14: CVRP instances: summary

\begin{tabular}{|c|c|c|c|c|c|c|c|c|c|c|c|c|c|c|c|}
\hline \multirow[b]{2}{*}{ Class } & \multirow[b]{2}{*}{$N P$} & \multicolumn{3}{|c|}{ BMR } & \multicolumn{3}{|c|}{$\mathrm{BCM}$} & \multicolumn{5}{|c|}{ FLL } & \multicolumn{3}{|c|}{ LLE } \\
\hline & & $O p t$ & $\% L B$ & $T$ & $O p t$ & $\% L B$ & $T$ & $O p t$ & $O p t_{B C P}$ & $O p t_{B C}$ & $\% L B$ & $T$ & $O p t$ & $\% L B$ & $T$ \\
\hline A & 22 & 22 & 99.9 & 30 & 22 & 99.8 & 118 & 22 & 20 & 2 & 99.2 & 1,961 & 15 & 97.9 & 6,638 \\
\hline B & 20 & 20 & 99.9 & 67 & 20 & 99.8 & 417 & 20 & 6 & 14 & 99.5 & 4,763 & 19 & 99.4 & 8,178 \\
\hline E-M & 12 & 9 & 99.8 & 303 & 8 & 99.4 & 1,025 & 9 & 7 & 2 & 98.9 & 126,987 & 3 & 97.7 & 39,592 \\
\hline $\mathrm{F}$ & 3 & 2 & 100.0 & 164 & & & & 3 & 0 & 3 & 99.9 & 2,398 & 3 & 99.9 & 1,046 \\
\hline $\mathrm{P}$ & 24 & 24 & 99.8 & 85 & 22 & 99.7 & 187 & 24 & 16 & 8 & 99.2 & 2,892 & 16 & 97.7 & 11,219 \\
\hline Avg & & & 99.9 & 92 & & 99.7 & 323 & & & & 99.3 & 17,409 & & 98.4 & 9,935 \\
\hline Tot & 81 & 77 & & & 72 & & & 78 & 49 & 29 & & & 56 & & \\
\hline
\end{tabular}


TABLE 4.15: CVRP: impact of parameter $\Delta\left(N_{i}\right)$ and of different types of inequalities

\begin{tabular}{|c|c|c|c|c|c|c|c|c|c|c|c|c|c|c|c|c|c|c|c|}
\hline \multirow[b]{3}{*}{ Inst } & \multirow[b]{3}{*}{$z^{*}$} & \multicolumn{4}{|c|}{$H^{1}$} & \multicolumn{8}{|c|}{$H^{2}$} & \multicolumn{4}{|c|}{$H^{3}$} & \multicolumn{2}{|c|}{$H^{4}$} \\
\hline & & \multicolumn{2}{|c|}{ no CCs } & \multicolumn{2}{|c|}{$\mathrm{CCs}$} & \multicolumn{2}{|c|}{$\Delta\left(N_{i}\right)=5$} & \multicolumn{2}{|c|}{$\Delta\left(N_{i}\right)=8$} & \multicolumn{2}{|c|}{$\Delta\left(N_{i}\right)=10$} & \multicolumn{2}{|c|}{$\Delta\left(N_{i}\right)=12$} & \multicolumn{2}{|c|}{ no WSR3s } & \multicolumn{2}{|c|}{ WSR3s } & \multirow[b]{2}{*}{$L B_{4}$} & \multirow[b]{2}{*}{$T$} \\
\hline & & $L B_{1}$ & $T$ & $L B_{1}$ & $T$ & $L B_{2}$ & $T$ & $L B_{2}$ & $T$ & $L B_{2}$ & $T$ & $L B_{2}$ & $T$ & $L B_{3}$ & $T$ & $L B_{3}$ & $T$ & & \\
\hline B-n50-k8 & $1,312.0$ & $1,229.0$ & 2 & $1,291.1$ & 11 & $1,302.6$ & 20 & $1,302.6$ & 21 & $1,302.6$ & 21 & $1,302.6$ & 22 & $1,302.6$ & 24 & $1,303.0$ & 39 & $1,308.8$ & 118 \\
\hline B-n66-k9 & $1,316.0$ & $1,223.8$ & 3 & $1,301.7$ & 17 & 307.0 & 20 & $1,307.4$ & 22 & $1,307.4$ & 23 & $1,307.4$ & 24 & $1,307.7$ & 27 & $1,309.7$ & 70 & $1,315.2$ & 283 \\
\hline B-n68-k9 & $1,272.0$ & $1,166.8$ & 3 & $1,261.1$ & 23 &, 263.2 & 28 & $1,263.2$ & 29 & $1,263.2$ & 29 & $1,263.2$ & 31 & $1,263.2$ & 34 & $1,264.3$ & 103 & $1,267.6$ & 336 \\
\hline B-n78-k10 & $1,221.0$ & $1,126.4$ & 4 & $1,208.4$ & 23 & $1,212.7$ & 27 & $1,213.9$ & 29 & $1,214.3$ & 30 & $1,214.5$ & 32 & $1,214.9$ & 36 & $1,218.0$ & 67 & $1,221.0$ & 91 \\
\hline E-n76-k7 & 682.0 & 667.2 & 2 & 667.2 & 5 & 668.5 & 20 & 668.9 & 22 & 669.2 & 23 & 669.3 & 23 & 669.8 & 28 & 673.3 & 74 & 680.6 & 151 \\
\hline E-n76-k8 & 735.0 & 720.3 & 2 & 724.3 & 13 & 724.7 & 33 & 724.8 & 34 & 724.9 & 36 & 724.9 & 38 & 725.1 & 45 & 729.1 & 72 & 734.0 & 81 \\
\hline E-n76-k10 & 830.0 & 811.3 & 1 & 815.4 & 18 & 815.9 & 46 & 815.9 & 46 & 816.1 & 49 & 816.2 & 52 & 816.5 & 54 & 820.9 & 73 & 826.2 & 81 \\
\hline E-n76-k14 & $1,021.0$ & 999.2 & 1 & $1,004.3$ & 15 & $1,006.1$ & 26 & $1,006.7$ & 28 & $1,007.0$ & 33 & $1,007.0$ & 36 & $1,007.0$ & 36 & $1,009.3$ & 40 & $1,014.7$ & 42 \\
\hline E-n101-k8 & 815.0 & 796.5 & 5 & 801.8 & 30 & 802.1 & 32 & 802.1 & 32 & 802.4 & 34 & 802.4 & 35 & 806.4 & 35 & 808.8 & 218 & 815.0 & 579 \\
\hline E-n101-k14 & $1,067.0$ & $1,044.6$ & 5 & $1,049.6$ & 10 & $1,047.5$ & 33 & $1,047.6$ & 35 & $1,047.7$ & 40 & $1,047.8$ & 40 & $1,052.9$ & 45 & $1,056.7$ & 191 & $1,062.3$ & 319 \\
\hline F-n135-k7 & $1,162.0$ & $1,098.2$ & 140 & $1,158.0$ & 1,057 & $1,158.9$ & 1,359 & $1,159.1$ & 1,396 & $1,159.3$ & 1,405 & $1,159.3$ & 1,423 & - & & - & & - & \\
\hline M-n121-k7 & $1,034.0$ & $1,013.2$ & 9 & $1,028.5$ & 94 & $1,031.1$ & 173 & $1,031.8$ & 178 & $1,032.0$ & 191 & $1,032.0$ & 197 & $1,032.2$ & 220 & $1,032.5$ & 611 & $1,033.4$ & 1,247 \\
\hline P-n50-k8 & 631.0 & 612.3 & $<0.1$ & 613.4 & 6 & 614.6 & 10 & 615.3 & 11 & 615.5 & 13 & 615.9 & 13 & 615.9 & 15 & 618.6 & 27 & 625.1 & 64 \\
\hline P-n55-k10 & 694.0 & 676.9 & 1 & 677.8 & 5 & 679.7 & 9 & 680.6 & 11 & 680.6 & 12 & 680.6 & 12 & 680.6 & 15 & 682.6 & 25 & 689.1 & 19 \\
\hline P-n70-k10 & 827.0 & 807.9 & 1 & 811.5 & 13 & 812.3 & 24 & 812.9 & 28 & 813.2 & 31 & 813.3 & 32 & 813.6 & 34 & 817.6 & 60 & 823.0 & 150 \\
\hline P-n76-k5 & 627.0 & 614.1 & 3 & 614.1 & 5 & 615.3 & 24 & 615.7 & 27 & 615.9 & 27 & 616.1 & 28 & 616.8 & 32 & 619.5 & 87 & 627.0 & 282 \\
\hline P-n101-k4 & 681.0 & 668.5 & 13 & 675.7 & 55 & 676.0 & 58 & 676.0 & 59 & 676.4 & 60 & 676.4 & 60 & 676.9 & 62 & 678.7 & 73 & 681.0 & 1,154 \\
\hline Avg & & 96.4 & & 98.5 & & 98.7 & & 98.7 & & 98.8 & & 98.8 & & 98.8 & & 99.1 & & 99.7 & \\
\hline
\end{tabular}




\section{Chapter 5}

\section{Multi-Trip Vehicle Routing Problem}

1

The multi-trip vehicle routing problem (MTVRP) is a variant of the capacitated vehicle routing problem where each vehicle can perform a subset of routes, called a vehicle schedule, subject to maximum driving time constraints. Despite its practical importance, the MTVRP has received little attention in the literature. Few heuristics have been proposed, and only an exact algorithm has been presented for a variant of the MTVRP with customer time window constraints and unlimited driving time for each vehicle. We describe two set-partitioning-like formulations of the MTVRP. The first formulation requires the generation of all feasible routes, whereas the second formulation is based on the generation of all feasible schedules. We study valid lower bounds, based on the linear relaxations of both formulations enforced with valid inequalities, that are embedded into an exact solution method. The computational results show that the proposed exact algorithm can solve MTVRP instances taken from the literature, with up to 120 customers.

\subsection{Introduction}

The capacitated vehicle routing problem (CVRP) and its many variations play an important role in the management of many distribution systems. In most of the studied models, the vehicles are identical and each vehicle is allowed to perform, at most, a single route.

In many contexts, a distribution company uses leased vehicles to service the customers and incurs a significant cost for each vehicle used. Whenever the planning period

\footnotetext{
${ }^{1}$ This chapter is based on Mingozzi et al. [2012]
} 
is large with respect to the route duration, and some vehicles can perform several routes in the period, the primary concern of the company is to minimize the number of vehicles used. The multi-trip vehicle routing problem (MTVRP) is an extension of the CVRP where each vehicle can perform multiple routes during its working period. The MTVRP can be described as follows.

A complete undirected graph $G=\left(V^{\prime}, E\right)$ is given, where $V^{\prime}$ is the set of vertices and $E$ is the set of edges. We have $V^{\prime}=\{0\} \cup V$, where vertex 0 represents the depot and the set $V=\{1, \ldots, n\}$ represents $n$ customers, each one requiring $q_{i}$ units of product from the depot. A fleet $M=\{1, \ldots, m\}$ of $m$ identical vehicles is located at the depot. Each vehicle has capacity $Q$ and maximum driving time $T$. A travel cost $\alpha_{i j}$ and a travel time $\tau_{i j}$ are associated with each edge $\{i, j\} \in E$. All input data are assumed to be nonnegative.

A route of a vehicle is a least-cost elementary cycle in $G$ that passes through the depot and a subset of the customers such that the total demand of the customers visited does not exceed the vehicle capacity $Q$. The cost (duration) of a route is equal to the sum of the travel costs (travel times) of the edges traversed.

A schedule of a vehicle is a subset of routes whose total duration is less than or equal to the maximum driving time $T$. The cost of a schedule is equal to the sum of the costs of its routes.

The MTVRP calls for the design of a set of $m$ schedules of minimum total cost such that each customer is visited exactly once by the routes of the schedules.

\subsection{Literature Review}

To the best of our knowledge, no exact algorithm for the MTVRP has been presented in the literature so far. The main heuristic methods proposed are surveyed in the following. Fleischmann [1990] addressed the problem first. He proposed a modification of the well-known saving algorithm and by using a bin packing heuristic to assign the routes to the vehicles. Taillard et al. [1996] proposed a three-phase algorithm. In the first phase, a set of routes satisfying the capacity constraints are designed. Next, the routes are combined to derive different CVRP solutions. Finally, the routes of each CVRP solution are assigned to the vehicles by solving a bin packing problem. Petch and Salhi [2004] described a multi-phase heuristic that constructs many feasible CVRP solutions and, for each one, assigns routes to vehicles with a bin packing heuristic. Salhi and Petch [2007] investigated a genetic algorithm approach. Olivera and Viera [2007] described an adaptive memory heuristic algorithm. Different tabu search algorithms were proposed by Alonso et al. [2008], Brandão and Mercer [1997, 1998]. A real life application of the MTVRP is described in Gribkovskaia et al. [2006]. 
Azi et al. [2010] addressed a variant of the MTVRP where: (i) a time window and a revenue are associated with each customer, (ii) the duration of each route is limited, (iii) the maximum driving time of the vehicles is unlimited, and (iv) it is not required to service all customers. Thus, the customers must be chosen based on their associated revenue minus the traveling cost to reach them. Azi et al. described a branch-and-price algorithm based on a set packing formulation that can routinely solve instances with 25 customers and a few instances with up to 50 customers. When the time windows are removed, the problem becomes the well-known distance-constrained CVRP. In this case, the algorithm was able to solve two instances with 25 customers.

\subsection{Mathematical Formulations and Relaxations}

In this section, we describe two set-partitioning-like formulations, called $F 1$ and $F 2$, and a valid integer relaxation, called $R F 1$, of the MTVRP. Formulation $F 1$ requires the a priori generation of all feasible routes, whereas formulation $F 2$ is based on the generation of all feasible schedules. Relaxation $R F 1$ is an IP problem similar to the set partitioning formulation of the CVRP.

\subsubsection{Formulation $F 1$}

Let $\mathscr{R}$ be the index set of all feasible routes on graph $G$, and let $\mathscr{R}_{i} \subseteq \mathscr{R}$ be the index subset of the routes visiting customer $i \in V$. A cost $d_{\ell}$ and a duration $\tau_{\ell}$ are associated with each route $\ell \in \mathscr{R}$. In the following, we use $R_{\ell}$ and $E\left(R_{\ell}\right)$ to indicate the set of customers visited and the edges traversed by route $\ell \in \mathscr{R}$, respectively.

Let $\xi_{\ell}^{j}$ be a binary variable equal to 1 if and only if route $\ell \in \mathscr{R}$ is assigned to vehicle $j \in M$. The mathematical formulation $F 1$ is as follows

$$
\begin{aligned}
& \text { (F1) } \quad z(F 1)=\min \sum_{\ell \in \mathscr{R}} d_{\ell} \sum_{j \in M} \xi_{\ell}^{j} \\
& \text { s.t. } \sum_{\ell \in \mathscr{R}_{i}} \sum_{j \in M} \xi_{\ell}^{j}=1, \quad i \in V \text {, } \\
& \sum_{\ell \in \mathscr{R}} \tau_{\ell} \xi_{\ell}^{j} \leq T, \quad j \in M, \\
& \xi_{\ell}^{j} \in\{0,1\}, \quad j \in M, \ell \in \mathscr{R} .
\end{aligned}
$$

Constraints (5.2) impose that each customer is visited exactly once, and constraints (5.3) define a feasible schedule for each vehicle used.

We denote by $L F 1$ the LP-relaxation of formulation $F 1$ and by $z(L F 1)$ its optimal solution cost. 


\subsubsection{Formulation $F 2$}

Let $\mathscr{H}$ be the index set of all schedules. For each schedule $k \in \mathscr{H}$, we denote by $\Omega_{k} \subseteq \mathscr{R}$ the index subset of the routes in the schedule, by $c_{k}=\sum_{\ell \in \Omega_{k}} d_{\ell}$ its cost, and by $\tau\left(\Omega_{k}\right)=\sum_{\ell \in \Omega_{k}} \tau_{\ell}$ its total duration. Moreover, we denote by $V\left(\Omega_{k}\right)=\cup_{\ell \in \Omega_{k}} R_{\ell}$ and $E\left(\Omega_{k}\right)=\cup_{\ell \in \Omega_{k}} E\left(R_{\ell}\right)$ the set of customers visited and the set of edges traversed by schedule $k \in \mathscr{H}$, respectively.

We assume that $\mathscr{H}$ contains undominated schedules only (i.e., given $k \in \mathscr{H}$, there exists no $k^{\prime} \in \mathscr{H} \backslash\{k\}$ such that $V\left(\Omega_{k}\right)=V\left(\Omega_{k^{\prime}}\right)$ and $\left.c_{k}>c_{k^{\prime}}\right)$. Notice that whenever $m=1, \mathscr{H}$ contains only schedules corresponding to optimal integer solutions of the CVRP, where the total duration of the routes is less than or equal to $T$.

Let $y_{k}$ be a binary variable equal to 1 if and only if schedule $k \in \mathscr{H}$ is assigned to a vehicle. Formulation $F 2$ of the MTVRP is as follows

$$
\begin{aligned}
&(F 2) \quad z(F 2)=\min \sum_{k \in \mathscr{H}} c_{k} y_{k} \\
& \text { s.t. } \sum_{k \in \mathscr{H}: i \in V\left(\Omega_{k}\right)} y_{k}=1, \quad i \in V, \\
& \sum_{k \in \mathscr{H}} y_{k} \leq m, \\
& y_{k} \in\{0,1\}, \quad k \in \mathscr{H} .
\end{aligned}
$$

Constraints (5.6) specify that each customer $i \in V$ must be visited exactly once. Constraint (5.7) imposes the upper bound on the number of vehicles used.

We denote by $L F 2$ the LP-relaxation of $F 2$ and by $z(L F 2)$ its optimal solution cost.

\subsubsection{Comparing Relaxations $L F 1$ and $L F 2$}

The following proposition shows the relation between relaxations $L F 1$ and $L F 2$.

Proposition 1 . The inequality $z(L F 1) \leq z(L F 2)$ holds and can be strict.

Proof. We show that any optimal dual solution of $L F 1$ of cost $z(L F 1)$ is a feasible dual solution of $L F 2$ of cost $z(L F 1)$, as well. Let $\boldsymbol{u}=\left(u_{1}, \ldots, u_{n}\right)$ and $\boldsymbol{w}=\left(w_{1}, \ldots, w_{m}\right)$ 
be the vectors of the dual variables associated with constraints (5.2) and (5.3), respectively. The dual of $L F 1$ is

$$
\begin{array}{cr}
(D F 1) \quad z(D F 1)=\max & \sum_{i \in V} u_{i}+T \sum_{j \in M} w_{j} \\
\text { s.t. } & \sum_{i \in R_{\ell}} u_{i}+\tau_{\ell} w_{j} \leq d_{\ell}, \quad j \in M, \ell \in \mathscr{R}, \\
u_{i} \in \mathbb{R}, & i \in V, \\
w_{j} \leq 0, & j \in M .
\end{array}
$$

Consider a vehicle schedule $\Omega_{k}$. Adding inequalities (5.10) over the routes of $\Omega_{k}$ and all vehicles of $M$ and dividing by $m$, we obtain

$$
\sum_{\ell \in \Omega_{k}} \sum_{i \in R_{\ell}} u_{i}+\sum_{\ell \in \Omega_{k}} \frac{\tau_{\ell}}{m} \sum_{j \in M} w_{j} \leq \sum_{\ell \in \Omega_{k}} d_{\ell} .
$$

Define $\bar{u}_{0}=\frac{T}{m} \sum_{j \in M} w_{j}$. Because $\sum_{\ell \in \Omega_{k}} \tau_{\ell} \leq T$ and $w_{j} \leq 0$, we have

$$
\bar{u}_{0} \leq \sum_{\ell \in \Omega_{k}} \frac{\tau_{\ell}}{m} \sum_{j \in M} w_{j} \leq 0 .
$$

From inequalities (5.13) and (5.14), we derive

$$
\sum_{\ell \in \Omega_{k}} \sum_{i \in R_{\ell}} u_{i}+\bar{u}_{0} \leq c_{k},
$$

which corresponds to the dual constraint associated with variable $y_{k}$ of problem $L F 2$, thus showing that $\overline{\boldsymbol{u}}=\left(\bar{u}_{0}, u_{1}, \ldots, u_{n}\right)$ is a dual solution of $L F 2$, where $u_{i}, i \in V$, are the dual variables of constraints (5.6) and $\bar{u}_{0}$ is the dual variable of constraint (5.7). The cost of the dual solution $\overline{\boldsymbol{u}}$ of $L F 2$ defined above is

$$
\sum_{i \in V} u_{i}+m \bar{u}_{0}=\sum_{i \in V} u_{i}+\frac{m T}{m} \sum_{j \in M} w_{j}=z(L F 1) \cdot \square
$$

The following example shows that $z(L F 1)$ can be strictly smaller than $z(L F 2)$.

Example 1. Consider an MTVRP instance with $n=4, m=2, T=100, Q=2$, $q_{1}=2, q_{2}=1, q_{3}=1$, and $q_{4}=2$. Travel costs and times coincide; in particular, we have $\alpha_{01}=\tau_{01}=30, \alpha_{02}=\tau_{02}=15, \alpha_{03}=\tau_{03}=15, \alpha_{04}=\tau_{04}=30$, and $\alpha_{23}=\tau_{23}=20$.

Formulation $F 1$ has the following five feasible routes (i.e., $|\mathscr{R}|=5$ ) for each vehicle: $R_{1}=\{1\}, d_{1}=60 ; R_{2}=\{2\}, d_{2}=30 ; R_{3}=\{3\}, d_{3}=30 ; R_{4}=\{4\}, d_{4}=60 ;$ and $R_{5}=\{2,3\}, d_{5}=50$. Solving $L F 1$, we obtain $z(L F 1)=170$ corresponding to $\xi_{1}^{1}=1$, $\xi_{5}^{1}=\frac{1}{2}, \xi_{4}^{2}=1, \xi_{5}^{2}=\frac{1}{2}$ (the other $\xi_{\ell}^{j}$ variables having a null value). 
Formulation $F 2$ has the following nine schedules (i.e., $|\mathscr{H}|=9$ ): $\Omega_{1}=\{1\}, c_{1}=60$; $\Omega_{2}=\{2\}, c_{2}=30 ; \Omega_{3}=\{3\}, c_{3}=30 ; \Omega_{4}=\{4\}, c_{4}=60 ; \Omega_{5}=\{5\}, c_{5}=50 ;$ $\Omega_{6}=\{1,2\}, c_{6}=90 ; \Omega_{7}=\{1,3\}, c_{7}=90 ; \Omega_{8}=\{2,4\}, c_{8}=90 ;$ and $\Omega_{9}=\{3,4\}$, $c_{9}=90$. Solving $L F 2$, we obtain $z(L F 2)=180>z(L F 1)$ corresponding to solution $y_{6}=1, y_{9}=1$, and $y_{k}=0$, for any $k \neq 6,9$.

\subsubsection{Relaxation $R F 1$}

Problem $R F 1$ is an integer problem derived from $F 1$ and is used, in the bounding procedures described in the next sections, to compute three lower bounds to the MTVRP. Problem $R F 1$ is obtained from $F 1$ as follows.

1. Replace constraints (5.3) with the following surrogate constraint

$$
\sum_{\ell \in \mathscr{R}} \tau_{\ell} \sum_{j \in M} \xi_{\ell}^{j} \leq m T
$$

2. Set $x_{\ell}=\sum_{j \in M} \xi_{\ell}^{j}, \ell \in \mathscr{R}$. Notice that, because of constraints (5.2), we have $x_{\ell} \in\{0,1\}$.

3. Replace the term $\sum_{j \in M} \xi_{\ell}^{j}$ with $x_{\ell}$ into expressions (5.1), (5.2), and (5.15).

The resulting relaxed problem $R F 1$ involves only the binary variables $x_{\ell}, \ell \in \mathscr{R}$, where $x_{\ell}$ is equal to 1 if and only if route $\ell$ is in the solution.

$$
\begin{aligned}
(R F 1) \quad z(R F 1)=\min & \sum_{\ell \in \mathscr{R}} d_{\ell} x_{\ell} \\
\text { s.t. } & \sum_{\ell \in \mathscr{R}_{i}} x_{\ell}=1, \quad i \in V, \\
& \sum_{\ell \in \mathscr{R}} \tau_{\ell} x_{\ell} \leq m T, \\
& \\
& x_{\ell} \in\{0,1\}, \quad \ell \in \mathscr{R} .
\end{aligned}
$$

In the following, we refer to problem $R F 1$ as the CVRP relaxation of the MTVRP (or as the CVRP associated to the MTVRP) and denote by $L R F 1$ the LP-relaxation of $R F 1$ and by $z(L R F 1)$ its optimal solution cost.

Proposition 2. The following equality holds

$$
z(L F 1)=z(L R F 1)
$$

Proof. Equality (5.20) follows from the observation that any $L F 1$ solution $\boldsymbol{\xi}$ can be transformed into an $L R F 1$ solution $\boldsymbol{x}$ of the same cost, and vice versa. 
The following algorithm transforms a solution $\boldsymbol{x}$ of $L R F 1$ into a feasible $L F 1$ solution $\boldsymbol{\xi}$ of $\operatorname{cost} z(L R F 1)$.

1. Initialize $j=0$.

2. Set $j=j+1$ and $w t=0$.

3. Let $\ell^{*}=\min \left\{\ell \in \mathscr{R}: x_{\ell^{*}}>0\right\}$. If $\tau_{\ell^{*}} x_{\ell^{*}} \leq T-w t$, set $w t=w t+\tau_{\ell^{*}} x_{\ell^{*}}$, $\xi_{\ell^{*}}^{j}=x_{\ell^{*}}, x_{\ell^{*}}=0$, and repeat Step 3. If $\tau_{\ell^{*}} x_{\ell^{*}}>T-w t$, define $p=\frac{(T-w t)}{\tau_{\ell^{*}} x_{\ell^{*}}}$, and set $\xi_{\ell^{*}}^{j}=p, x_{\ell^{*}}=1-p$, and return to Step 2 .

Any $L F 1$ solution $\boldsymbol{\xi}$ can be transformed into a feasible solution $\boldsymbol{x}$ of $L R F 1$ of cost $z(L F 1)$ by simply setting $x_{\ell}=\sum_{j \in M} \xi_{\ell}^{j}$.

\subsection{Improving Relaxations $L R F 1$ and $L F 2$ and Outline of the Exact Method}

In this section, we describe two relaxations, called $\overline{L R F 1}$ and $\overline{L F 2}$, derived respectively from $L R F 1$ and $L F 2$ by adding some families of valid inequalities. These relaxations are used to derive valid lower bounds on the MTVRP that are embedded in the exact method proposed in $\S 5.8$.

\subsubsection{Relaxation $\overline{L R F 1}$}

Relaxation $\overline{L R F 1}$ derives from $L R F 1$ by adding two types of valid inequalities for the set partitioning formulation of the CVRP (see Chapter 4), called strengthened capacity inequalities and subset row inequalities, and replacing inequality (5.18) with a new type of inequalities, called working time inequalities.

Strengthened Capacity Inequalities. Let $\mathscr{S}$ be the set of all the subsets of customers of cardinality greater than or equal to 2 (i.e., $\mathscr{S}=\{S \subseteq V:|S| \geq 2\}$ ), and let $\delta(S)$ be the cutset of $S \in \mathscr{S}$. Let $\rho_{\ell}(S)$ be the number of edges of $\delta(S)$ traversed by route $\ell \in \mathscr{R}$ (i.e., $\left.\rho_{\ell}(S)=\left|E\left(R_{\ell}\right) \cap \delta(S)\right|\right)$. The strengthened capacity (SC) inequalities are

$$
\sum_{\ell \in \mathscr{R}} \rho_{\ell}(S) x_{\ell} \geq 2 k(S), \quad S \in \mathscr{S}
$$

where $k(S)$ is a lower bound on the number of vehicles required to service the customer set $S$ and can be computed as $\left\lceil\sum_{i \in S} q_{i} / Q\right\rceil$.

Subset Row Inequalities. Subset row (SR) inequalities were introduced by Jepsen et al. [2008] for the vehicle routing problem with time windows. They combine clique inequalities with odd-hole inequalities. Let $\mathscr{C}=\{C \subseteq V:|C| \geq 3\}$, and let $\eta$ be 
an integer such that $\eta>1$. Any feasible $R F 1$ solution must satisfy the following SR inequality

$$
\sum_{\ell \in \mathscr{R}} \varphi_{\ell}(C) x_{\ell} \leq \varphi_{0}(C), \quad C \in \mathscr{C}
$$

where $\varphi_{\ell}(C)=\left\lfloor\frac{\left\lfloor R_{\ell} \cap C \mid\right.}{\eta}\right\rfloor$, and $\varphi_{0}(C)=\left\lfloor\frac{|C|}{\eta}\right\rfloor$. We consider the following three special cases of the SR inequalities where $\eta=2$.

1. SR3 inequalities. SR3 inequalities correspond to the subset $\mathscr{C}^{3} \subseteq \mathscr{C}$ defined as $\mathscr{C}^{3}=\{C \subseteq V:|C|=3\}$. SR3 inequalities correspond to a subset of clique inequalities and impose that, at most, one of the routes visiting at least two of the customers in $C \in \mathscr{C}^{3}$ can be in the solution.

2. SR5 inequalities. SR5 inequalities correspond to the subset $\mathscr{C}^{5} \subseteq \mathscr{C}$ defined as $\mathscr{C}^{5}=\{C \subseteq V:|C|=5\}$. SR5 inequalities correspond to odd-hole inequalities and impose that, at most, two of the routes visiting at least two of the customers in $C \in \mathscr{C}^{5}$ can be in the solution.

3. WSR3 inequalities. Weak subset row (WSR3) inequalities, introduced in $\S 4.3 .2$, are downlifted SR3 inequalities. They are used as an alternative to the SR3 inequalities in the bounding procedures described in $\S 5.5$. Let $E(C)$, $C \in \mathscr{C}^{3}$, be the edges whose terminal vertices are both in $C$. The coefficients of the WSR3 inequalities, $\varphi_{\ell}(C)$, are computed as $\varphi_{\ell}(C)=1$ if $\left|E\left(R_{\ell}\right) \cap E(C)\right| \geq 1$, and $\varphi_{\ell}(C)=0$ if $\left|E\left(R_{\ell}\right) \cap E(C)\right|=0$.

Hereafter, we use $\mathscr{C}=\mathscr{C}^{3} \cup \mathscr{C}^{5}$ and $C \in \mathscr{C}$ to refer to both the index and the customer subset of an SR inequality. The SR3, SR5, and WSR3 inequalities are separated through complete enumeration.

Working Time Inequalities. These new inequalities strengthen inequality (5.18). They are given by the following proposition.

Proposition 3. Let $t_{\min }$ be a lower bound on the duration of any route $\ell \in \mathscr{R}$. If travel costs $\alpha_{i j}$ satisfy the triangle inequality, we can define $t_{\text {min }}=\min _{i \in V}\left\{2 \tau_{0 i}\right\}$. For a given time $t \in\left[t_{\min }, T\right]$, let $\hat{t}=\operatorname{Tmod}(t)+1$, and let $\mathscr{L}=\left\{L \subseteq \mathscr{R}: \tau_{\ell} \in[\hat{t}, t)\right.$ for each $\ell \in$ $L$, and $R_{\ell^{\prime}} \cap R_{\ell^{\prime \prime}} \neq \varnothing$, for each $\left.\ell^{\prime}, \ell^{\prime \prime} \in L\right\}$. The following inequalities, called working time (WT) inequalities, are valid for $L R F 1$

$$
\sum_{\ell \in \mathscr{R} \backslash L}\left\lfloor\frac{\tau_{\ell}}{t}\right\rfloor x_{\ell}+\sum_{\ell \in L} x_{\ell} \leq\left\lfloor\frac{T}{t}\right\rfloor m, \quad t \in\left[t_{\text {min }}, T\right], L \in \mathscr{L} .
$$

Proof. For a given $L \in \mathscr{L}$, inequalities (5.3) can be written as

$$
\sum_{\ell \in \mathscr{R} \backslash L} \tau_{\ell} \xi_{\ell}^{j} \leq T-\sum_{\ell \in L} \tau_{\ell} \xi_{\ell}^{j}, \quad j \in M
$$


Inequalities (5.22) can be relaxed by replacing $\tau_{\ell}$ with $\hat{t}$, for each route $\ell \in L$

$$
\sum_{\ell \in \mathscr{R} \backslash L} \tau_{\ell} \xi_{\ell}^{j} \leq T-\hat{t} \sum_{\ell \in L} \xi_{\ell}^{j}, \quad j \in M .
$$

By dividing inequalities (5.23) by $t$ and rounding down the coefficients, we have

$$
\sum_{\ell \in \mathscr{R} \backslash L}\left\lfloor\frac{\tau_{\ell}}{t}\right\rfloor \xi_{\ell}^{j} \leq\left\lfloor\frac{T-\hat{t} \sum_{\ell \in L} \xi_{\ell}^{j}}{t}\right\rfloor, \quad j \in M .
$$

Notice that because of the definition of the set $L, \sum_{\ell \in L} \xi_{\ell}^{j}$ is equal to either 0 or 1 for any feasible MTVRP solution. If $\sum_{\ell \in L} \xi_{\ell}^{j}=0$, then $\left\lfloor\frac{T-\hat{t} \sum_{\ell \in L} \xi_{\ell}^{j}}{t}\right\rfloor=\left\lfloor\frac{T}{t}\right\rfloor$. If $\sum_{\ell \in L} \xi_{\ell}^{j}=1$, because of the definition of $\hat{t}$, we have $\left\lfloor\frac{T-\hat{t} \sum_{\ell \in L} \xi_{\ell}^{j}}{t}\right\rfloor=\left\lfloor\frac{T-\hat{t}}{t}\right\rfloor=\left\lfloor\frac{T}{t}\right\rfloor-1$. Thus, we have

$$
\left\lfloor\frac{T-\hat{t} \sum_{\ell \in L} \xi_{\ell}^{j}}{t}\right\rfloor=\left\lfloor\frac{T}{t}\right\rfloor-\sum_{\ell \in L} \xi_{\ell}^{j}, \quad j \in M .
$$

From inequalities (5.24) and equations (5.25), we derive

$$
\sum_{\ell \in \mathscr{R} \backslash L}\left\lfloor\frac{\tau_{\ell}}{t}\right\rfloor \xi_{\ell}^{j}+\sum_{\ell \in L} \xi_{\ell}^{j} \leq\left\lfloor\frac{T}{t}\right\rfloor, \quad j \in M .
$$

By summing up inequalities (5.26) and replacing $\sum_{j \in M} \xi_{\ell}^{j}$ with $x_{\ell} \in\{0,1\}$, we derive inequalities (5.21).

The separation of inequalities (5.21) is $\mathcal{N} \mathcal{P}$-hard because it requires the computation of the set $\mathscr{L}$. Therefore, we use a subset of the WT inequalities (5.21) that are obtained by defining $L_{i t}=\left\{\ell \in \mathscr{R}_{i}: \tau_{\ell} \in[\hat{t}, t)\right\}$, for each vertex $i \in V$, and by setting $\mathscr{L}=\left\{L_{i t}: i \in V, t \in\left[t_{\text {min }}, T\right]\right\}$. Let $\mathscr{W}=\left\{(t, i): t \in\left[t_{\text {min }}, T\right], i \in V\right\}$. These latter inequalities can be conveniently written as follows

$$
\sum_{\ell \in \mathscr{R}} \vartheta_{\ell}(t, i) x_{\ell} \leq \vartheta_{0}(t), \quad(t, i) \in \mathscr{W}
$$

where, for a given $(t, i) \in \mathscr{W}, \vartheta_{\ell}(t, i)=\left\lfloor\frac{\tau_{\ell}}{t}\right\rfloor$, for each route $\ell \in \mathscr{R} \backslash L_{i t}, \vartheta_{\ell}(t, i)=1$, for each route $\ell \in L_{i t}$, and $\vartheta_{0}(t)=\left\lfloor\frac{T}{t}\right\rfloor m$. Inequalities (5.27) can be separated by complete enumeration.

Example 2. Consider the MTVRP instance defined in Example 1 of $§ 5.3 .3$. Problem $R F 1$ involves the same set $\mathscr{R}$ of routes of formulation $F 1$. An optimal solution of $R F 1$ of cost $z(R F 1)=z(L R F 1)=170$ is given by $x_{1}=1, x_{4}=1$, and $x_{5}=1$. Consider the WT inequality (5.27) for $t=60$ and $i=3$. As $\hat{t}=41$, we have $L_{60,3}=\{5\}$. Thus, the coefficients of $(5.27)$ are $\vartheta_{1}(60,30)=1, \vartheta_{2}(60,30)=0, \vartheta_{3}(60,30)=0, \vartheta_{4}(60,30)=1$, $\vartheta_{5}(60,30)=1$, and $\vartheta_{0}(60)=2$. Inequality (5.27) corresponds to $x_{1}+x_{4}+x_{5} \leq 2$, which is violated by the aforementioned solution of $R F 1$. 
Relaxation $\overline{L R F 1}$ is the following problem

$$
\begin{array}{rlr}
(\overline{L R F 1}) \quad z(\overline{L R F 1})=\min & \sum_{\ell \in \mathscr{R}} d_{\ell} x_{\ell} & \\
\text { s.t. } & \sum_{\ell \in \mathscr{R}_{i}} x_{\ell}=1, & i \in V, \\
& \sum_{\ell \in \mathscr{R}} \rho_{\ell}(S) x_{\ell} \geq 2 k(S), & S \in \mathscr{S}, \\
& \sum_{\ell \in \mathscr{R}} \varphi_{\ell}(C) x_{\ell} \leq \varphi_{0}(C), \quad C \in \mathscr{C}, \\
& \sum_{\ell \in \mathscr{R}} \vartheta_{\ell}(t, i) x_{\ell} \leq \vartheta_{0}(t), \quad(t, i) \in \mathscr{W}, \\
& x_{\ell} \geq 0, & \ell \in \mathscr{R} .
\end{array}
$$

Let $(\boldsymbol{u}, \boldsymbol{v}, \boldsymbol{g}, \boldsymbol{h})$ be the dual variables of $\overline{L R F 1}$, where $\boldsymbol{u} \in \mathbb{R}^{n}$ is associated with (5.29), $\boldsymbol{v} \in \mathbb{R}_{+}^{|\mathscr{S}|}$ with (5.30), $\boldsymbol{g} \in \mathbb{R}_{-}^{|\mathscr{C}|}$ with (5.31), and $\boldsymbol{h} \in \mathbb{R}_{-}^{|\mathscr{W}|}$ with (5.32).

In $\S 5.5$, we describe three bounding procedures to solve $\overline{L R F 1}$, called $H^{1}, H^{2}$, and $H^{3}$, that are extensions of similar procedures proposed in Chapter 4 for the CVRP. The three procedures are executed in sequence, and the dual solution of procedure $H^{k}$ is used to hot-start procedure $H^{k+1}, k=1,2$.

Procedures $H^{1}$ and $H^{2}$ are dual-ascent heuristics based on a cut-and-column generation (CCG) method that differs from standard CCG methods based on the simplex for the use of a dual-ascent heuristic to find a near-optimal dual solution of problem $\overline{L R F 1}$. Procedure $H^{1}$ finds a dual solution $\left(\boldsymbol{u}^{\mathbf{1}}, \boldsymbol{v}^{\mathbf{1}}, \boldsymbol{g}^{\mathbf{1}}\right)$ of cost $L B_{1}$ of problem $\overline{L R F 1}$ by adding SC and WSR3 inequalities, ignoring WT inequalities, and replacing the route set $\mathscr{R}$ with the set of all $q$-routes (see Christofides et al. [1981b]). Procedure $H^{2}$ differs from $H^{1}$ as it uses elementary routes instead of $q$-routes. Procedure $H^{2}$ achieves a dual solution $\left(\boldsymbol{u}^{\mathbf{2}}, \boldsymbol{v}^{\mathbf{2}}, \boldsymbol{g}^{\mathbf{2}}\right)$ of cost $L B_{2}$, such that $L B_{1} \leq L B_{2}$. Procedure $H^{3}$ is a CCG method based on the simplex to solve problem $\overline{L R F 1}$. Procedure $H^{3}$ inherits from $H^{2}$ the master and achieves an optimal dual solution $\left(\boldsymbol{u}^{\mathbf{3}}, \boldsymbol{v}^{\mathbf{3}}, \boldsymbol{g}^{\mathbf{3}}, \boldsymbol{h}^{\mathbf{3}}\right)$ of cost $L B_{3}$. 


\subsubsection{Relaxation $\overline{L F 2}$}

Relaxation $\overline{L F 2}$ is obtained by adding SC, SR3, and SR5 inequalities to LF2

$$
\begin{aligned}
&(\overline{L F 2}) \quad z(\overline{L F 2})=\min \sum_{k \in \mathscr{H}} c_{k} y_{k} \\
& \text { s.t. } \sum_{k \in \mathscr{H}: i \in V\left(\Omega_{k}\right)} y_{k}=1, \quad i \in V, \\
& \sum_{k \in \mathscr{H}} y_{k} \leq m, \\
& \sum_{k \in \mathscr{H}} \rho_{k}(S) y_{k} \geq 2 k(S), \quad S \in \mathscr{S}, \\
& \sum_{k \in \mathscr{H}} \varphi_{k}(C) y_{k} \leq \varphi_{0}(C), \quad C \in \mathscr{C}, \\
& y_{k} \geq 0, \\
& k \in \mathscr{H},
\end{aligned}
$$

where $\rho_{k}(S)$ and $\varphi_{k}(C)$ of constraints (5.37) and (5.38) are computed as $\rho_{k}(S)=$ $\left|E\left(\Omega_{k}\right) \cap \delta(S)\right|$ and $\varphi_{k}(C)=\left\lfloor\frac{\left|V\left(\Omega_{k}\right) \cap C\right|}{\eta}\right\rfloor$.

Let $(\boldsymbol{w}, \boldsymbol{v}, \boldsymbol{g})$ be the dual variables of $\overline{L F 2}$, where $\boldsymbol{w}=\left(w_{0}, w_{1}, \ldots, w_{n}\right)$ and $w_{0} \in \mathbb{R}_{-}$is associated with constraint $(5.36),\left(w_{1}, \ldots, w_{n}\right) \in \mathbb{R}^{n}$ with constraints $(5.35), \boldsymbol{v} \in \mathbb{R}_{+}^{|\mathscr{S}|}$ with constraints (5.37), and $\boldsymbol{g} \in \mathbb{R}_{-}^{|\mathscr{C}|}$ with constraints (5.38).

In $\S 5.6$, we describe a CCG procedure, called $H^{4}$, to solve $\overline{L F 2}$. We denote by $\boldsymbol{x}^{4}$ and $\left(\boldsymbol{w}^{\mathbf{4}}, \boldsymbol{v}^{\mathbf{4}}, \boldsymbol{g}^{\mathbf{4}}\right)$ the optimal primal and dual solutions, respectively, of $\overline{L F 2}$ of cost $L B_{4}$ achieved by $H^{4}$.

\subsubsection{Using Multiple Dual Solutions to Improve Procedures $H^{2}, H^{3}$ and $H^{4}$}

In this section, we describe two simple methods to improve the lower bounds and the computing times of procedures $H^{2}, H^{3}$ and $H^{4}$.

The method used in $H^{2}$ and $H^{3}$ is based on the following simple observation which applies to any problem with binary variables. Consider the following problem with $n$ variables and $m$ constraints

$$
\begin{aligned}
&(F) \quad z(F)=\min \boldsymbol{c} \boldsymbol{x} \\
& \text { s.t. } \boldsymbol{A x}=\boldsymbol{b}, \\
& \boldsymbol{x} \in\{0,1\}^{n} .
\end{aligned}
$$

Let us denote by $L F$ the LP-relaxation of problem $F$ and by $D$ the dual of $L F$, and let $z(F), z(L F)$ and $z(D)$ be the optimal solution costs of $F, L F$ and $D$, respectively. We assume to know a valid upper bound $z_{U B}$ on $z(F)$. 
Observation 1. Let $\boldsymbol{w}^{\prime}$ be a feasible (nonnecessarily optimal) $D$ solution of cost $z^{\prime}(D)$. Because any optimal $F$ solution $\boldsymbol{x}^{*}$ satisfies $z(F)=z^{\prime}(D)+\sum_{j \in J} c_{j}^{\prime}$, where $c_{j}^{\prime}=c_{j}-$ $\boldsymbol{w}^{\prime} \boldsymbol{a}_{j}, J=\left\{j: x_{j}^{*}=1,1 \leq j \leq n\right\}$ is the reduced cost of variable $x_{j}$ and $a_{j}$ is the $j$ th column vector of matrix $\boldsymbol{A}$, then any variable $x_{j}$ such that $z^{\prime}(D)+c_{j}^{\prime}>z_{U B}$ cannot be in any optimal solution of cost less than or equal to $z_{U B}$ and can be removed from $F$. The resulting problem $F^{\prime}$ has the same optimal solutions of $F$. Let $L F^{\prime}$ be the LP-relaxation of $F^{\prime}$, and let $z\left(L F^{\prime}\right)$ be its optimal solution cost. It follows that $z\left(L F^{\prime}\right) \geq z(L F)$, and such inequality can be strict if $c_{j}^{\prime}>z_{U B}-z(L F)$ for some variable $x_{j}$ of the optimal basis of $L F$. In practice, it might happen that $z(L F)<z\left(L F^{\prime}\right)=z(F)$, as shown in the following example.

Example 3. Consider the following set partitioning problem $F$

\begin{tabular}{|c|c|c|c|c|c|c|c|}
\hline $\begin{array}{l}\min = \\
\text { s.t. }\end{array}$ & $\begin{array}{l}x_{1} \\
x_{1}\end{array}$ & $+x_{2}$ & $+x_{3}$ & $\begin{array}{c}+4 x_{4} \\
+x_{4}\end{array}$ & $\begin{array}{c}+3 x_{5} \\
+x_{5}\end{array}$ & $\begin{array}{c}+4.5 x_{6} \\
+x_{6}\end{array}$ & $=1$, \\
\hline & & $x_{2}$ & & $+x_{4}$ & $+x_{5}$ & $+x_{6}$ & $=1$, \\
\hline & & & $x_{3}$ & $+x_{4}$ & & $+x_{6}$ & $=1$, \\
\hline & $x_{1}$ & $+x_{2}$ & & & $+x_{5}$ & & $=1$, \\
\hline & $x_{1}$ & & $+x_{3}$ & & & $+x_{6}$ & $=1$, \\
\hline & & $x_{2}$ & $+x_{3}$ & & & & $=1$, \\
\hline
\end{tabular}

A valid upper bound is $z_{U B}=4.5$, whereas the optimal integer solution cost of $F$ is $z(F)=4$ corresponding to $x_{3}=1, x_{5}=1, x_{j}=0, j \neq 3,5$. The optimal $L F$ solution cost is $z(L F)=2$ corresponding to $\boldsymbol{x}=\left(\frac{1}{2}, \frac{1}{2}, \frac{1}{2}, \frac{1}{2}, 0,0\right)$. Consider the dual solution $\boldsymbol{w}=\left(\frac{1}{3}, \frac{1}{3}, \frac{1}{3}, \frac{1}{3}, \frac{1}{3}, \frac{1}{3}\right)$ of cost $z(D)=2$; the vector $\boldsymbol{c}^{\prime}$ of the reduced costs with respect to $\boldsymbol{w}$ is $\boldsymbol{c}^{\prime}=\left(0,0,0,3,2, \frac{19}{6}\right)$.

According to Observation 1, we can remove variables $x_{4}$ and $x_{6}$ from $F$ as $z(D)+c_{4}^{\prime}>$ $z_{U B}$ and $z(D)+c_{6}^{\prime}>z_{U B}$. Thus, we derive problem $F^{\prime}$ that involves variables $x_{1}, x_{2}$, $x_{3}$ and $x_{5}$ only.

An optimal solution of $L F^{\prime}$ is $\boldsymbol{x}=(0,0,1,0,1,0)$ of cost $z\left(L F^{\prime}\right)=4$, which is also an optimal integer solution of $F$; that is $z(L F)<z\left(L F^{\prime}\right)=z(F)$.

Observation 1 is used by procedures $H^{2}$ and $H^{3}$ for solving the pricing problem. Procedure $H^{k}, k=2,3$, uses the dual solution $\left(\boldsymbol{u}^{\boldsymbol{k}-\mathbf{1}}, \boldsymbol{v}^{\boldsymbol{k}-\mathbf{1}}, \boldsymbol{g}^{\boldsymbol{k}-\mathbf{1}}\right)$ produced by $H^{k-1}$ to reject any route of negative reduced cost with respect to the incumbent dual solution of problem $\overline{L R F 1}$ but having reduced cost with respect to $\left(\boldsymbol{u}^{\boldsymbol{k - 1}}, \boldsymbol{v}^{\boldsymbol{k - 1}}, \boldsymbol{g}^{\boldsymbol{k}-\mathbf{1}}\right)$ greater than $z_{U B}-L B_{k-1}$, where $z_{U B}$ is a valid upper bound to the MTVRP that can be computed by running any of the heuristic algorithms available from the literature (hereafter, we assume to know such upper bound on the MTVRP). 
In practice, this method improves both the final lower bound $L B_{k}$ achieved by $H^{k}$ and the computing time of $H^{k}$, avoiding the generation of routes of negative reduced cost that cannot be in any optimal integer solution.

To improve the lower bound value as well as the computing time of $H^{4}$, we use the following observation.

Observation 2. Let $d_{\ell}^{3}$ be the reduced cost of route $\ell \in \mathscr{R}$ with respect to the dual solution $\left(\boldsymbol{u}^{\mathbf{3}}, \boldsymbol{v}^{\mathbf{3}}, \boldsymbol{g}^{\mathbf{3}}, \boldsymbol{h}^{\mathbf{3}}\right)$ of $\overline{L R F 1}$ of cost $L B_{3}$ achieved by $H^{3}$. Let $z(F 2)$ be the cost of an optimal $F 2$ solution $\boldsymbol{y}^{*}$, and let $Y=\left\{k \in \mathscr{H}: y_{k}^{*}=1\right\}$. The following inequality holds $z(F 2) \geq L B_{3}+\sum_{k \in Y} d^{3}\left(\Omega_{k}\right)$, where $d^{3}\left(\Omega_{k}\right)=\sum_{\ell \in \Omega_{k}} d_{\ell}^{3}$. Thus, any schedule $k \in \mathscr{H}$ such that $d^{3}\left(\Omega_{k}\right)>z_{U B}-L B_{3}$ cannot be in any optimal solution and can be removed from $F 2$.

Observation 2 is used in procedure $H^{4}$ to reject any schedule $\Omega_{k}$ of negative reduced cost with respect to the dual solution of the master such that $d^{3}\left(\Omega_{k}\right)>z_{U B}-L B_{3}$.

\subsection{Bounding Procedures $H^{1}, H^{2}$, and $H^{3}$ Based on Re- laxation $\overline{L R F 1}$}

In this section, we describe three bounding procedures $H^{1}, H^{2}$ and $H^{3}$ to derive lower bounds $L B_{1}, L B_{2}$ and $L B_{3}$, respectively. Procedures $H^{1}$ and $H^{2}$ use a method, called CCG, proposed by Baldacci et al. [2008] for the CVRP and used in Chapter 4 for solving the VRPTW and the CVRP. Procedure $H^{3}$ is a CCG method, based on the simplex, that uses a new strategy to solve the pricing problem.

\subsubsection{Algorithm CCG}

This algorithm differs from standard CCG methods based on the simplex as it uses a dual-ascent heuristic to find a near-optimal dual solution of the master problem. The main advantages of this method are

1. it is faster than simplex based methods;

2. it is not affected by the typical degeneration of the simplex;

3. the dual solution produced does not correspond to a dual basic solution of the master, thus making the pricing problem easier to solve.

The Dual Heuristic to Solve the Master Problem. The master problem of $\overline{L R F 1}$ with SC and WSR3 inequalities is defined by a subset $\overline{\mathscr{R}} \subseteq \mathscr{R}$ and the sets $\mathscr{S}$ and $\mathscr{C}$ of SC and WSR3 inequalities. The initializations of the sets $\overline{\mathscr{R}}, \mathscr{S}$ and $\mathscr{C}$ are 
described in $\S \S 5.5 .2$ and 5.5.4. The dual-ascent heuristic to compute a feasible dual solution $(\overline{\boldsymbol{u}}, \overline{\boldsymbol{v}}, \overline{\boldsymbol{g}})$ of the master problem corresponds to the method described in $\S 4.5 .1$ and is based on the following theorem.

Theorem 5.1. Let us associate penalties $\lambda_{i} \in \mathbb{R}, i \in V$, with constraints (5.29), $\mu_{S} \in \mathbb{R}_{+}, S \in \mathscr{S}$, with $S C$ constraints (5.30), and $\omega_{C} \in \mathbb{R}_{-}, C \in \mathscr{C}$, with constraints (5.31) in the form of WSR3 inequalities. For each route $R_{\ell}(\ell \in \mathscr{R})$, we indicate with $\bar{c}_{\ell}$ its reduced cost with respect to $(\boldsymbol{\lambda}, \boldsymbol{\mu}, \boldsymbol{\omega})$ (i.e., $\bar{c}_{\ell}=c_{\ell}-\sum_{i \in R_{\ell}} \lambda_{i}-\sum_{S \in \mathscr{S}} \rho_{\ell}(S) \mu_{S}-$ $\left.\sum_{C \in \mathscr{C}} \varphi_{\ell}(C) \omega_{C}\right)$. For each $i \in V$, let us compute

$$
\beta_{i}=q_{i} \min _{\ell \in \overline{\mathscr{R}}: i \in R_{\ell}}\left\{\frac{\bar{c}_{\ell}}{\sum_{i \in R_{\ell}} q_{i}}\right\} .
$$

A feasible dual solution $(\boldsymbol{u}, \boldsymbol{v}, \boldsymbol{g})$ of the master of cost $z(\overline{\operatorname{LRF} 1}(\boldsymbol{\lambda}, \boldsymbol{\mu}, \boldsymbol{\omega}))$ is obtained by setting

$$
u_{i}=\beta_{i}+\lambda_{i}, i \in V, \quad v_{S}=\mu_{S}, S \in \mathscr{S}, \quad \text { and } \quad g_{C}=\omega_{C}, C \in \mathscr{C} .
$$

Proof. See $§ 3.5 .2$.

A near-optimal dual solution $(\overline{\boldsymbol{u}}, \overline{\boldsymbol{v}}, \overline{\boldsymbol{g}})$ of the current master problem is obtained by an iterative procedure that performs a predefined number Maxit2 of subgradient iterations to solve the problem

$$
L C C G=\max _{(\boldsymbol{\lambda}, \boldsymbol{\mu}, \boldsymbol{\omega})}\{z(\overline{L R F 1}(\boldsymbol{\lambda}, \boldsymbol{\mu}, \boldsymbol{\omega}))\}
$$

We denote by $(\overline{\boldsymbol{u}}, \overline{\boldsymbol{v}}, \overline{\boldsymbol{g}})$ the near-optimal dual solution of $\overline{L R F 1}$ achieved after Maxit2 iterations and by $\overline{\boldsymbol{x}}$ the corresponding nonnecessarily feasible solution of $\overline{L R F 1}$.

Adding violated WSR3 inequalities and solving the pricing problem. After computing the master dual solution $(\overline{\boldsymbol{u}}, \overline{\boldsymbol{v}}, \overline{\boldsymbol{g}})$, CCG adds, to the master, the largest subset $\mathscr{C}^{\prime}$ of at most $\Delta(\mathscr{C})$ WSR3 inequalities most violated by $\overline{\boldsymbol{x}}$, where $\Delta(\mathscr{C})$ is a given parameter. In our computational results, we set $\Delta(\mathscr{C})=100$. The set $\mathscr{S}$ of $\mathrm{SC}$ inequalities is generated at the beginning of CCG, as described in Baldacci et al. [2008], and is not changed.

Moreover, CCG generates a subset $\mathscr{N} \subseteq \mathscr{R}$ of routes of negative reduced cost with respect to $(\overline{\boldsymbol{u}}, \overline{\boldsymbol{v}}, \overline{\boldsymbol{g}})$. If $\mathscr{N} \neq \varnothing$, then CCG sets $\widehat{\mathscr{R}}=\widehat{\mathscr{R}} \cup \mathscr{N}$; otherwise, $(\overline{\boldsymbol{u}}, \overline{\boldsymbol{v}}, \overline{\boldsymbol{g}})$ is a feasible dual solution.

CCG terminates after Maxit1 macro iterations and provides an $\overline{L R F 1}$ dual solution, $\left(\boldsymbol{u}^{*}, \boldsymbol{v}^{*}, \boldsymbol{g}^{*}\right)$, of cost $L C C G$ corresponding to the penalty vector $\left(\boldsymbol{\lambda}^{*}, \boldsymbol{\mu}^{*}, \boldsymbol{\omega}^{*}\right)$. 


\subsubsection{Bounding Procedure $H^{1}$}

Procedure $H^{1}$ enlarges the route set $\mathscr{R}$ with an extension of the $q$-routes introduced by Christofides et al. [1981b]. The initial route set $\overline{\mathscr{R}}$ of the master problem contains all single-customer routes $(0, i, 0), i \in V$. We initialize $\mathscr{C}=\varnothing$ and $(\boldsymbol{\lambda}, \boldsymbol{\mu}, \boldsymbol{\omega})=(\mathbf{0}, \mathbf{0}, \mathbf{0})$.

At a given macro iteration, the set $\mathscr{N}$ of routes of negative reduced cost with respect to the dual solution $(\overline{\boldsymbol{u}}, \overline{\boldsymbol{v}}, \overline{\boldsymbol{g}})$ is computed as follows. Define the modified arc cost

$$
\bar{\alpha}_{i j}=\alpha_{i j}-\frac{1}{2}\left(\bar{u}_{i}+\bar{u}_{j}\right)-\sum_{S \in \mathscr{S}:\{i, j\} \in \delta(S)} \bar{v}_{S}, \quad \text { for each edge }\{i, j\} \in E,
$$

with respect to $(\overline{\boldsymbol{u}}, \overline{\boldsymbol{v}}, \overline{\boldsymbol{g}})$.

A $(q, j, i)$-path is a nonnecessarily elementary path that starts from the depot and visits a subset of customers (once or more) such that their total demand is $q$ and the last two customers visited are $j$ and $i$ in this order. A $(q, j, i)$-path is defined for any load $q\left(q_{j}+q_{i} \leq q \leq Q\right)$, and for any pair of vertices $j, i \in V^{\prime}, j \neq i$. A $(q, j, 0)$-route (or simply a $q$-route) is a $(q, j, 0)$-path. Let $f(q, j, i)$ be the cost of a least-cost $(q, j, i)$-path using the modified arc costs $\bar{\alpha}_{i j}$, and let $\mathscr{C}_{i j}=\{C \in \mathscr{C}:\{i, j\} \in E(C)\}$. Functions $f(q, j, i)$ can be computed with the following dynamic programming (DP) recursion

$$
\begin{aligned}
f(q, j, i)=\min _{k \in V^{\prime} \backslash\{i\}}\left\{f\left(q-q_{i}, k, j\right)+\bar{\alpha}_{j i}-\sum_{C \in \mathscr{C}_{j i} \backslash \mathscr{C}_{k j}} \bar{g}_{C}\right\}, \\
q=q_{j}+q_{i}, \ldots, Q, \quad j, i \in V^{\prime}, j \neq i, j \neq 0 .
\end{aligned}
$$

Functions $f(q, j, i)$ are initialized as $f\left(q_{i}, 0, i\right)=\bar{\alpha}_{0 i}, i \in V$.

The set $\mathscr{N}$ contains any $q$-route of negative reduced cost corresponding to a least-cost $q$-route visiting customer $j \in V$ as the last customer before arriving at the depot.

At the end, procedure $H^{1}$ sets $\left(\boldsymbol{u}^{\mathbf{1}}, \boldsymbol{v}^{\mathbf{1}}, \boldsymbol{g}^{\mathbf{1}}\right)=\left(\boldsymbol{u}^{*}, \boldsymbol{v}^{*}, \boldsymbol{g}^{*}\right),\left(\boldsymbol{\lambda}^{\mathbf{1}}, \boldsymbol{\mu}^{\mathbf{1}}, \boldsymbol{\omega}^{\mathbf{1}}\right)=\left(\boldsymbol{\lambda}^{*}, \boldsymbol{\mu}^{*}, \omega^{*}\right)$, and $L B_{1}=L C C G$.

\subsubsection{Route Generation Algorithm GenR}

In this section, we describe the two-phase algorithm GenR used by bounding procedure $H^{2}$ to initialize the master problem and to solve the pricing problem. Moreover, the second phase of GenR is used by $H^{3}$ to solve the pricing problem and by the exact method to generate all routes of reduced cost, with respect to $\left(\boldsymbol{u}^{\mathbf{3}}, \boldsymbol{v}^{\mathbf{3}}, \boldsymbol{g}^{\mathbf{3}}, \boldsymbol{h}^{\mathbf{3}}\right)$, less than $z_{U B}-L B_{3}$. Algorithm GenR generates elementary routes and extends the method proposed by Baldacci et al. [2008] for the CVRP by introducing new bounding functions to reduce the state-space graph. GenR is based on the following observations. 
Let $V(P) \subseteq V$ be the subset of customers visited by path $P$, and let $E(P)$ be the edges traversed by path $P$. Let $\mathscr{P}$ be the set of all elementary paths of minimum cost from the depot such that $q(P) \leq \frac{Q}{2}+q_{\sigma(P)}$, for each path $P \in \mathscr{P}$, where $q(P)=\sum_{i \in V(P)} q_{i}$, $\tau(P)=\sum_{\{i, j\} \in E(P)} \tau_{i j}$, and $\sigma(P)$ represent the load, the duration and the terminal customer of path $P$, respectively. We denote by $\pi(P)$ the immediate predecessor of $\sigma(P)$ in $P$.

Every route passing through customer $i \in V$ can be obtained by combining a pair of paths $P, \bar{P} \in \mathscr{P}$ such that

$\sigma(P)=\sigma(\bar{P})=i, \quad V(P) \cap V(\bar{P})=\{i\}, \quad \tau(P)+\tau(\bar{P}) \leq T, \quad q(P)+q(\bar{P}) \leq Q+q_{i}$.

Given a nonnecessarily feasible dual solution $(\tilde{\boldsymbol{u}}, \tilde{\boldsymbol{v}}, \tilde{\boldsymbol{g}})$ of $\overline{L R F 1}$, a feasible dual solution $\left(\boldsymbol{u}^{\prime}, \boldsymbol{v}^{\prime}, \boldsymbol{g}^{\prime}\right)$ of $\overline{L R F 1}$ and four parameters $\Delta(\mathscr{P}), \Delta(\mathscr{R}), \gamma$ and $\gamma^{\prime}$, GenR generates the largest subset $\mathscr{B}$ of routes such that $|\mathscr{B}| \leq \Delta(\mathscr{R}), \tilde{d}_{\ell} \leq \gamma$ and $d_{\ell}^{\prime} \leq \gamma^{\prime}$, for each route $\ell \in \mathscr{B}$, where $\tilde{d}_{\ell}$ and $d_{\ell}^{\prime}$ are the reduced costs of route $\ell$ with respect to $(\tilde{\boldsymbol{u}}, \tilde{\boldsymbol{v}}, \tilde{\boldsymbol{g}})$ and $\left(\boldsymbol{u}^{\prime}, \boldsymbol{v}^{\prime}, \boldsymbol{g}^{\prime}\right)$, respectively. In the first phase, GenR generates the path set $\mathscr{P}$ such that $|\mathscr{P}| \leq \Delta(\mathscr{P})$, where $\Delta(\mathscr{P})$ is a parameter that limits the cardinality of the set $\mathscr{P}$ and is imposed for memory limits. In the second phase, GenR combines the paths of $\mathscr{P}$ to provide the subset $\mathscr{B} \subseteq \mathscr{R}$. Parameters $\Delta(\mathscr{R}), \gamma$ and $\gamma^{\prime}$ are defined according to the type of subset $\mathscr{B}$ that must be generated.

Define the modified path cost $\tilde{d}(P)$ with respect to $(\tilde{\boldsymbol{u}}, \tilde{\boldsymbol{v}})$ as follows

$$
\tilde{d}(P)=\sum_{\{i, j\} \in E(P)} \tilde{\alpha}_{i j}
$$

where $\tilde{\alpha}_{i j}=\alpha_{i j}-\frac{1}{2}\left(\tilde{u}_{i}+\tilde{u}_{j}\right)-\sum_{S \in \mathscr{S}:\{i, j\} \in \delta(S)} \tilde{v}_{S}$. Let $l b(P)$ be a lower bound on the reduced cost with respect to $(\tilde{\boldsymbol{u}}, \tilde{\boldsymbol{v}}, \tilde{\boldsymbol{g}})$ of any route containing path $P$. For a given path $P \in \mathscr{P}$, let $\sigma(P)=i$. The lower bound $l b(P)$ is computed as

$l b(P)=\tilde{d}(P)-\sum_{C \in \mathscr{C}}\left\lceil\frac{|E(P) \cap E(C)|}{2} \mid \tilde{g}_{C}+\min _{j \in V \backslash V(P), q_{i} \leq q^{\prime} \leq Q-q(P)+q_{i}}\left\{f\left(q^{\prime}, j, i\right)+\tilde{g}_{\pi(P) i j}\right\}\right.$,

where $\tilde{g}_{\pi(P) i j}$ is the dual of the WSR3 inequality $\{\pi(P), i, j\} \in \mathscr{C}$ (we assume $\tilde{g}_{\pi(P) i j}=$ 0 if $\{\pi(P), i, j\} \notin \mathscr{C})$.

The two phases of GenR are shortly described as follows. For a more detailed description of GenR see Baldacci et al. [2008].

\subsubsection{Phase 1 of GenR.}

Phase 1 generates a sequence $\mathscr{P}$ of paths $\left(P^{1}, \ldots, P^{k}\right)$, with $k \leq \Delta(\mathscr{P})$, such that $l b\left(P^{1}\right) \leq \ldots \leq l b\left(P^{k}\right) \leq \gamma$. If Phase 1 terminates with $|\mathscr{P}|=\Delta(\mathscr{P})$, the entire 
algorithm terminates prematurely as there is no guarantee that all routes of reduced cost less than or equal to $\gamma$ are generated by combining the pairs $P, \bar{P} \in \mathscr{P}$.

\subsubsection{Phase 2 of GenR.}

Phase 2 is executed only if $|\mathscr{P}|<\Delta(\mathscr{P})$. Consider a pair of paths $(P, \bar{P}) \in \mathscr{P}$ satisfying conditions (5.40). Let $\ell \in \mathscr{R}$ be the index of the route resulting from $(P, \bar{P})$ of reduced cost $\tilde{d}_{\ell}$ with respect to $(\tilde{\boldsymbol{u}}, \tilde{\boldsymbol{v}}, \tilde{\boldsymbol{g}})$. From $(5.41)$, we have $\tilde{d}_{\ell}=\tilde{d}(P)+\tilde{d}(\bar{P})-$ $\sum_{C \in \mathscr{C}} \varphi_{\ell}(C) \tilde{g}_{C}$. As $\tilde{\boldsymbol{g}} \leq 0$, then $\tilde{d}(P)+\tilde{d}(\bar{P}) \leq \tilde{d}_{\ell}$, so only pairs of paths $(P, \bar{P})$ such that $\tilde{d}(P)+\tilde{d}(\bar{P}) \leq \gamma$ can generate routes of reduced cost less than or equal to $\gamma$.

Phase 2 dynamically generates a sequence of path pairs $\left(P^{r_{1}}, \bar{P}^{s_{1}}\right), \ldots,\left(P^{r_{k}}, \bar{P}^{s_{k}}\right)$, $\ldots,\left(P^{r_{h}}, \bar{P}^{s_{h}}\right)$, where each path pair satisfies conditions (5.40) and $\tilde{d}\left(P^{r_{1}}\right)+\tilde{d}\left(\bar{P}^{s_{1}}\right) \leq$ $\ldots \leq \tilde{d}\left(P^{r_{k}}\right)+\tilde{d}\left(\bar{P}^{s_{k}}\right) \leq \ldots \leq \tilde{d}\left(P^{r_{h}}\right)+\tilde{d}\left(\bar{P}^{s_{h}}\right) \leq \gamma$. The pool $\mathscr{B}$ of routes generated contains any route $\ell \in \mathscr{R}$ resulting from the pairs of paths in the sequence such that $\tau_{\ell} \leq T, \tilde{d}_{\ell} \leq \gamma, d_{\ell}^{\prime} \leq \gamma^{\prime}$ and route $\ell$ is not dominated by any other route $\ell^{\prime}$ previously generated (i.e., route $\ell^{\prime}$ dominates route $\ell$ if $R_{\ell^{\prime}}=R_{\ell}$ and $\tilde{d}_{\ell^{\prime}} \leq \tilde{d}_{\ell}$ ).

\subsubsection{Bounding Procedure $H^{2}$}

Procedure $H^{2}$ is a straightforward implementation of CCG, where $\mathscr{R}$ is the set of elementary routes. The initial route set $\overline{\mathscr{R}}$ is obtained by executing GenR by setting $(\tilde{\boldsymbol{u}}, \tilde{\boldsymbol{v}}, \tilde{\boldsymbol{g}})=\left(\boldsymbol{u}^{\prime}, \boldsymbol{v}^{\prime}, \boldsymbol{g}^{\prime}\right)=\left(\boldsymbol{u}^{\mathbf{1}}, \boldsymbol{v}^{\mathbf{1}}, \boldsymbol{g}^{\mathbf{1}}\right), \Delta(\mathscr{P})=10^{7}, \Delta(\mathscr{R})=10^{4}$, and $\gamma=\gamma^{\prime}=$ $z_{U B}-L B_{1}$, and adding all single-customer routes to $\overline{\mathscr{R}}=\mathscr{B}$. Procedure $H^{2}$ starts with the same sets $\mathscr{S}$ and $\mathscr{C}$ of SC and WSR3 inequalities resulting from $H^{1}$. We initialize $(\boldsymbol{\lambda}, \boldsymbol{\mu}, \boldsymbol{\omega})=\left(\boldsymbol{\lambda}^{\mathbf{1}}, \boldsymbol{\mu}^{\mathbf{1}}, \boldsymbol{\omega}^{\mathbf{1}}\right)$.

At each macro iteration, a set $\mathscr{N}$ of routes of negative reduced cost with respect to $(\overline{\boldsymbol{u}}, \overline{\boldsymbol{v}}, \overline{\boldsymbol{g}})$ is given by $\mathscr{N}=\mathscr{B}$, where the set $\mathscr{B}$ is generated by GenR setting $(\tilde{\boldsymbol{u}}, \tilde{\boldsymbol{v}}, \tilde{\boldsymbol{g}})=$ $(\overline{\boldsymbol{u}}, \overline{\boldsymbol{v}}, \overline{\boldsymbol{g}}),\left(\boldsymbol{u}^{\prime}, \boldsymbol{v}^{\prime}, \boldsymbol{g}^{\prime}\right)=\left(\boldsymbol{u}^{\mathbf{1}}, \boldsymbol{v}^{\mathbf{1}}, \boldsymbol{g}^{\mathbf{1}}\right), \Delta(\mathscr{P})=10^{7}, \Delta(\mathscr{R})=150, \gamma=0$, and $\gamma^{\prime}=$ $z_{U B}-L B_{1}$.

At the end, $H^{2}$ sets $\left(\boldsymbol{u}^{\mathbf{2}}, \boldsymbol{v}^{\mathbf{2}}, \boldsymbol{g}^{\mathbf{2}}\right)=\left(\boldsymbol{u}^{*}, \boldsymbol{v}^{*}, \boldsymbol{g}^{*}\right),\left(\boldsymbol{\lambda}^{\mathbf{2}}, \boldsymbol{\mu}^{\mathbf{2}}, \boldsymbol{\omega}^{\mathbf{2}}\right)=\left(\boldsymbol{\lambda}^{*}, \boldsymbol{\mu}^{*}, \boldsymbol{\omega}^{*}\right)$, and $L B_{2}=L C C G$.

\subsubsection{Generating the Path Set $\mathscr{P}^{2}$ and the Route Set $\mathscr{R}^{2}$}

After the execution of $H^{2}$, we try to generate the route set $\mathscr{R}^{2}$ of all routes of reduced cost with respect to $\left(\boldsymbol{u}^{\mathbf{2}}, \boldsymbol{v}^{\mathbf{2}}, \boldsymbol{g}^{\mathbf{2}}\right)$ less than or equal to $z_{U B}-L B_{2}$. We call GenR by $\operatorname{setting}(\tilde{\boldsymbol{u}}, \tilde{\boldsymbol{v}}, \tilde{\boldsymbol{g}})=\left(\boldsymbol{u}^{\mathbf{2}}, \boldsymbol{v}^{\mathbf{2}}, \boldsymbol{g}^{\mathbf{2}}\right),\left(\boldsymbol{u}^{\prime}, \boldsymbol{v}^{\prime}, \boldsymbol{g}^{\prime}\right)=\left(\boldsymbol{u}^{\mathbf{1}}, \boldsymbol{v}^{\mathbf{1}}, \boldsymbol{g}^{\mathbf{1}}\right), \Delta(\mathscr{P})=10^{7}, \Delta(\mathscr{R})=5 \cdot 10^{6}$, $\gamma=z_{U B}-L B_{2}$, and $\gamma^{\prime}=z_{U B}-L B_{1}$. We denote by $\mathscr{P}^{2}$ and $\mathscr{R}^{2}$ the path set $\mathscr{P}$ and the route set $\mathscr{B}$ generated by GenR, respectively. Moreover, we denote by $d^{2}(P)$ 
the modified cost of path $P \in \mathscr{P}^{2}$ with respect to $\left(\boldsymbol{u}^{2}, \boldsymbol{v}^{2}\right)$ computed according to expression (5.41) by replacing $(\tilde{\boldsymbol{u}}, \tilde{\boldsymbol{v}})$ with $\left(\boldsymbol{u}^{\mathbf{2}}, \boldsymbol{v}^{\mathbf{2}}\right)$.

We define $\mathscr{P}^{2}$ to be optimal if $\left|\mathscr{P}^{2}\right|<\Delta(\mathscr{P})$ because it contains any path generating any route of any optimal MTVRP solution. We define $\mathscr{R}^{2}$ to be optimal if $\mathscr{P}^{2}$ is optimal and $\left|\mathscr{R}^{2}\right|<\Delta(\mathscr{R})$.

Because $H^{3}$ and the exact algorithm require that $\mathscr{P}^{2}$ be optimal, whenever $\mathscr{P}^{2}$ is not optimal (i.e., $\left|\mathscr{P}^{2}\right|=\Delta(\mathscr{P})$ ), $H^{3}$ is not executed and the entire procedure terminates prematurely.

\subsubsection{Bounding Procedure $H^{3}$}

Procedure $H^{3}$ is a column-and-cut-generation procedure, based on the simplex algorithm, to solve $\overline{L R F 1}$ with SC, SR3, SR5, and WT inequalities. $H^{3}$ differs from classical column-and-cut-generation algorithms because of the method used to solve the pricing problem.

$H^{3}$ uses sets $\mathscr{P}^{2}$ and $\mathscr{R}^{2}$, generated at the end of $H^{2}$, to generate routes of negative reduced cost. The route set $\overline{\mathscr{R}}$ of the master problem of $H^{3}$ is generated by extracting, from $\mathscr{R}^{2}$, the largest subset $\overline{\mathscr{R}}$ of routes of minimum reduced cost with respect to $\left(\boldsymbol{u}^{2}, \boldsymbol{v}^{2}, \boldsymbol{g}^{\mathbf{2}}\right)$ such that $|\widehat{\mathscr{R}}| \leq \Delta(\mathscr{B})$ and by adding all single-customer routes to $\overline{\mathscr{R}}$. The set $\mathscr{S}$ is set equal to the set of SC inequalities used in $H^{1}$ and $H^{2}$, and $\mathscr{W}$ and $\mathscr{C}$ are defined as $\mathscr{W}=\varnothing, \mathscr{C}=\varnothing$, where the set $\mathscr{C}=\mathscr{C}^{3} \cup \mathscr{C}^{5}$ represents SR3 and SR5 inequalities.

Let $\overline{\boldsymbol{x}}$ and $(\overline{\boldsymbol{u}}, \overline{\boldsymbol{v}}, \overline{\boldsymbol{g}}, \overline{\boldsymbol{h}})$ be, respectively, the primal and dual solutions of the master problem achieved at a given iteration. A set $\mathscr{N}$ of at most $\Delta(\mathscr{N})$ negative reduced cost routes with respect to $(\overline{\boldsymbol{u}}, \overline{\boldsymbol{v}}, \overline{\boldsymbol{g}}, \overline{\boldsymbol{h}})$ is generated with the following algorithm.

1. Extract, from $\mathscr{R}^{2}$, the largest subset $\mathscr{N}$ of at most $\Delta(\mathscr{N})$ routes having the largest negative reduced cost, and add them to $\overline{\mathscr{R}}$. If $\mathscr{N}=\varnothing$ and $\mathscr{R}^{2}$ is not optimal, continue with Step 2; otherwise, Stop.

2. Compute the modified cost $\bar{d}(P), P \in \mathscr{P}^{2}$, with respect to $(\overline{\boldsymbol{u}}, \overline{\boldsymbol{v}})$ according to (5.41) by replacing $(\tilde{\boldsymbol{u}}, \tilde{\boldsymbol{v}})$ with $(\overline{\boldsymbol{u}}, \overline{\boldsymbol{v}})$. Using the method described in $§ 5.5 .3 .2$, combine any pair of paths $P, P^{\prime} \in \mathscr{P}^{2}$ such that $d^{2}(P)+d^{2}\left(P^{\prime}\right) \leq z_{U B}-L B_{2}$ and $\bar{d}(P)+\bar{d}\left(P^{\prime}\right) \leq 0$ to derive the set $\mathscr{N}$.

At each iteration, $H^{3}$ adds, to $\mathscr{C}$, a set of at most $\Delta(\mathscr{C})$ most violated SR3 and SR5 inequalities and, to $\mathscr{W}$, a set of at most $\Delta(\mathscr{W})$ WT inequalities most violated by $\overline{\boldsymbol{x}}$. Procedure $H^{3}$ ends whenever $\mathscr{N}=\varnothing$ and no cuts are added, and it achieves an $\overline{L R F 1}$ dual solution $\left(\boldsymbol{u}^{\mathbf{3}}, \boldsymbol{v}^{\mathbf{3}}, \boldsymbol{g}^{\mathbf{3}}, \boldsymbol{h}^{\mathbf{3}}\right)$ of cost $L B_{3}$. 


\subsubsection{Generating the Route Set $\mathscr{R}^{3}$ and Solving $F 1$}

After the execution of $H^{3}$, we try to generate the largest set $\mathscr{R}^{3}$ of routes such that $d_{\ell}^{3} \leq z_{U B}-L B_{3}, \ell \in \mathscr{R}^{3}$, and $\left|\mathscr{R}^{3}\right| \leq \Delta(\mathscr{R})$, where $d_{\ell}^{3}$ is the reduced cost of route $\ell$ with respect to $\left(\boldsymbol{u}^{\mathbf{3}}, \boldsymbol{v}^{\mathbf{3}}, \boldsymbol{g}^{\mathbf{3}}, \boldsymbol{h}^{\mathbf{3}}\right)$. In generating $\mathscr{R}^{3}$ we have two cases

a. $\mathscr{R}^{2}$ is optimal. We set $\mathscr{R}^{3}=\left\{\ell \in \mathscr{R}^{2}: d_{\ell}^{3} \leq z_{U B}-L B_{3}\right\}$ and define $\mathscr{R}^{3}$ as optimal.

b. $\mathscr{R}^{2}$ is not optimal. Recall that in this case $\mathscr{P}^{2}$ is optimal, so $\mathscr{P}^{2}$ allows us to generate the routes of any optimal MTVRP solution. We compute the reduced cost $d^{3}(P)$ of each path $P \in \mathscr{P}^{2}$, with respect to $\left(\boldsymbol{u}^{\mathbf{3}}, \boldsymbol{v}^{\mathbf{3}}\right)$, according to expression (5.41) by replacing $(\tilde{\boldsymbol{u}}, \tilde{\boldsymbol{v}})$ with $\left(\boldsymbol{u}^{\mathbf{3}}, \boldsymbol{v}^{\mathbf{3}}\right)$. Then, we combine the path pairs of the set $\mathscr{P}^{2}$, by using the method of $\S 5.5 .3 .2$, to derive the set $\mathscr{R}^{3}$ of all routes such that $d_{\ell}^{2} \leq z_{U B}-L B_{3}$ and $d_{\ell}^{3} \leq z_{U B}-L B_{3}$, for each route $\ell \in \mathscr{R}^{3}$, and $\left|\mathscr{R}^{3}\right| \leq \Delta(\mathscr{R})$. If $\left|\mathscr{R}^{3}\right|<\Delta(\mathscr{R})$, then $\mathscr{R}^{3}$ is defined optimal.

If $\left|\mathscr{R}^{3}\right|$ is small (say, $\left|\mathscr{R}^{3}\right| \leq 5,000$ ), we find an optimal MTVRP solution by solving the reduced problem $F 1$, obtained by replacing route set $\mathscr{R}$ with $\mathscr{R}^{3}$, through an IP solver. Otherwise, we execute bounding procedure $H^{4}$ (described in $\S 5.6$ ).

\subsection{Bounding Procedure $H^{4}$ Based on Relaxation $\overline{L F 2}$}

Procedure $H^{4}$ is a column-and-cut generation method, based on the simplex, to solve relaxation $\overline{L F 2}$.

The initial sets $\mathscr{S}$ and $\mathscr{C}$ of SC, SR3 and SR5 inequalities are those resulting at the end of $H^{3}$. The initial master problem, $\overline{\mathscr{H}} \subseteq \mathscr{H}$, is defined as follows. Execute procedure GenSched (described in $\S 5.7$ ) to derive the schedule set $\mathscr{D}$ containing at most $\Delta(\mathscr{D})$ (where $\Delta(\mathscr{D})$ is a given parameter) schedules such that $c_{k}^{3} \leq z_{U B}-L B_{3}, k \in \mathscr{D}$, where $c_{k}^{3}$ is the reduced cost of schedule $k$ with respect to $\left(\boldsymbol{u}^{\mathbf{3}}, \boldsymbol{v}^{\mathbf{3}}, \boldsymbol{g}^{\mathbf{3}}, \boldsymbol{h}^{\mathbf{3}}\right)$ computed as $c_{k}^{3}=\sum_{\ell \in \Omega_{k}} d_{\ell}^{3}$. Set $\widehat{\mathscr{H}}=\mathscr{D}$, and add to $\widehat{\mathscr{H}}$ a schedule composed of a single-customer route for every customer $i$ not covered by any schedule of $\mathscr{D}$.

At each iteration, GenSched generates a set $\mathscr{N}$ of at most $\Delta(\mathscr{D})$ schedules of negative reduced cost with respect to the dual solution $(\overline{\boldsymbol{w}}, \overline{\boldsymbol{v}}, \overline{\boldsymbol{g}})$ of the master and such that $c_{k}^{3} \leq z_{U B}-L B_{3}$, for each schedule $k \in \mathscr{N}$. Moreover, the subset $\mathscr{C}^{\prime}$ of the $\Delta(\mathscr{C})$ SR3 and SR5 inequalities most violated by the current master solution are added to $\mathscr{C}$. In our computational experiments, we used $\Delta(\mathscr{D})=10^{4}$ in generating $\mathscr{D}, \Delta(\mathscr{D})=150$ in generating $\mathscr{N}$, and $\Delta(\mathscr{C})=100$ in generating $\mathscr{C}^{\prime}$.

Procedure $H^{4}$ terminates if $\mathscr{N}=\varnothing$ and $\mathscr{C}^{\prime}=\varnothing$ and provides a dual solution $\left(\boldsymbol{w}^{\mathbf{4}}, \boldsymbol{v}^{\mathbf{4}}, \boldsymbol{g}^{\mathbf{4}}\right)$ of $\operatorname{cost} L B_{4}$. 


\subsection{Algorithm GenSched to Generate Vehicle Schedules}

In this section, we describe procedure GenSched to generate the schedule sets $\overline{\mathscr{H}}$ and $\mathscr{N}$, which are required by bounding procedure $H^{4}$, and the schedule subset $\mathscr{H}^{4} \subseteq \mathscr{H}$, which is required by the exact method described in $\S 5.8$.

Given a dual solution $(\hat{\boldsymbol{w}}, \hat{\boldsymbol{v}}, \hat{\boldsymbol{g}})$ of $\overline{L F 2}$ and two parameters $\Delta(\mathscr{D})$ and $\gamma$, algorithm GenSched combines the routes of set $\mathscr{R}^{3}$ to produce the largest subset $\mathscr{D}$ of schedules of minimum reduced costs with respect to $(\hat{\boldsymbol{w}}, \hat{\boldsymbol{v}}, \hat{\boldsymbol{g}})$ such that $|\mathscr{D}| \leq \Delta(\mathscr{D}), c_{k}^{3} \leq$ $z_{U B}-L B_{3}$, and $\hat{c}_{k} \leq \gamma$, for each schedule $k \in \mathscr{D}$, where $\hat{c}_{k}$ is the reduced cost of schedule $k$ with respect to $(\hat{\boldsymbol{w}}, \hat{\boldsymbol{v}}, \hat{\boldsymbol{g}})$.

To generate the schedule sets $\overline{\mathscr{H}}, \mathscr{N}$ and $\mathscr{H}^{4}$, GenSched requires the following parameter settings

- The schedule set $\overline{\mathscr{H}}$ defining the initial master problem of $H^{4}$. Define $(\hat{\boldsymbol{w}}, \hat{\boldsymbol{v}}, \hat{\boldsymbol{g}})=$ $(\mathbf{0}, \mathbf{0}, \mathbf{0})$ and $\gamma=z_{U B}$.

- The set $\mathscr{N}$ of schedules of negative reduced costs in $H^{4}$. Define $(\hat{\boldsymbol{w}}, \hat{\boldsymbol{v}}, \hat{\boldsymbol{g}})$ as the dual of the current master problem of $H^{4}$ and $\gamma=0$.

- The schedule subset $\mathscr{H}^{4} \subseteq \mathscr{H}$ of all the schedules of any optimal MTVRP solution. Define $(\hat{\boldsymbol{w}}, \hat{\boldsymbol{v}}, \hat{\boldsymbol{g}})=\left(\boldsymbol{w}^{\mathbf{4}}, \boldsymbol{v}^{\mathbf{4}}, \boldsymbol{g}^{\mathbf{4}}\right)$ and $\gamma=z_{U B}-L B_{4}$.

Procedure GenSched is based on the following proposition.

Proposition 4. Let $\hat{d}_{\ell}=\sum_{\{i, j\} \in E\left(R_{\ell}\right)}\left(\alpha_{i j}-\frac{1}{2}\left(\hat{w}_{i}+\hat{w}_{j}\right)-\sum_{S \in \mathscr{S}:\{i, j\} \in \delta(S)} \hat{v}_{S}\right)-\sum_{C \in \mathscr{C}} \varphi_{\ell}(C) \hat{g}_{C}$, $\ell \in \mathscr{R}^{3}$, and let $\hat{d}\left(\Omega_{k}\right)=\sum_{\ell \in \Omega_{k}} \hat{d}_{\ell}, k \in \mathscr{H}$. The reduced cost $\hat{c}_{k}$ of schedule $k \in \mathscr{H}$ with respect to $(\hat{\boldsymbol{w}}, \hat{\boldsymbol{v}}, \hat{\boldsymbol{g}})$ satisfies the inequality $\hat{c}_{k} \geq \hat{d}\left(\Omega_{k}\right)$.

Proof. This happens as $w_{0} \leq 0, \hat{\boldsymbol{g}} \leq \mathbf{0}$, and $\varphi_{k}(C) \geq \sum_{\ell \in \Omega_{k}} \varphi_{\ell}(C)$, for any $C \in \mathscr{C}$ and any $k \in \mathscr{H}$.

Algorithm GenSched is a two-phase procedure. In the first phase, it computes the reduced costs $\hat{d}_{\ell}, \ell \in \mathscr{R}^{3}$, and orders the routes of the set $\mathscr{R}^{3}$ so that $\hat{d}_{\ell_{1}} \leq \hat{d}_{\ell_{2}} \leq$ $\ldots \leq \hat{d}_{\mid \mathscr{R}^{3}}$. The second phase is an iterative procedure that combines the routes $\mathscr{R}^{3}$ in schedules. At iteration $j$, an attempt is made to add the $j$ th route of the ordered set $\mathscr{R}^{3}$ to the schedules of a temporary schedule set POOL containing all the schedules composed of the first $j-1$ routes $\ell_{1}, \ell_{2}, \ldots, \ell_{j-1}$ of $\mathscr{R}^{3}$.

We assume that POOL is composed of undominated schedules, where schedule $k^{\prime}$ is dominated by schedule $k^{\prime \prime}$ if $V\left(\Omega_{k^{\prime \prime}}\right)=V\left(\Omega_{k^{\prime}}\right), \hat{d}\left(\Omega_{k^{\prime \prime}}\right) \leq \hat{d}\left(\Omega_{k^{\prime}}\right)$, and $\tau\left(\Omega_{k^{\prime \prime}}\right) \leq \tau\left(\Omega_{k^{\prime}}\right)$. A schedule $k^{\prime}$ that is obtained by adding route $\ell_{j}$ to a schedule $k \in$ POOL becomes a new member of POOL only if $\hat{d}\left(\Omega_{k^{\prime}}\right) \leq \gamma, k^{\prime}$ is not dominated by any other schedule $k^{\prime \prime} \in$ POOL, and $c^{3}\left(\Omega_{k^{\prime}}\right)=\sum_{\ell \in \Omega_{k^{\prime}}} d_{\ell}^{3} \leq z_{U B}-L B_{3}$. 
We denote by $p$ the number of schedules in POOL. The second phase of GenSched can be described as follows.

1. Initialize POOL $=\varnothing, \mathscr{D}=\varnothing$ and $p=0$.

2. For $\ell=1, \ldots,\left|\mathscr{R}^{3}\right|$ repeat Step 3 .

3. Expansion of the temporary schedule set $\mathrm{POOL}$ with route $\ell \in \mathscr{R}^{3}$. For each schedule $k \in$ POOL such that $V\left(\Omega_{k}\right) \cap R_{\ell}=\varnothing, \tau\left(\Omega_{k}\right)+\tau_{\ell} \leq T, c^{3}\left(\Omega_{k}\right)+d_{\ell}^{3} \leq$ $z_{U B}-L B_{3}$, and $\hat{d}\left(\Omega_{k}\right)+\hat{d}_{\ell} \leq \gamma$, repeat the following steps

- Define the schedule $\Omega^{\prime}=\Omega_{k} \cup\{\ell\}$ of duration $\tau\left(\Omega^{\prime}\right)=\tau\left(\Omega_{k}\right)+\tau_{\ell}$, and set $\hat{d}\left(\Omega^{\prime}\right)=\hat{d}(\Omega)+\hat{d}_{\ell}$.

- If $\Omega^{\prime}$ is not dominated by any other schedule $\Omega^{\prime \prime} \in$ POOL, insert $\Omega^{\prime} \in$ POOL and set $p=p+1$. Remove any schedule $\Omega^{\prime \prime}$ dominated by $\Omega^{\prime}$ from Pool, and update $p$, accordingly.

- Compute $\hat{c}\left(\Omega^{\prime}\right)$. If $\hat{c}\left(\Omega^{\prime}\right) \leq \gamma$, add $\Omega^{\prime}$ to $\mathscr{D}$. If $|\mathscr{D}|=p$, Stop.

\subsection{Description of the Exact Method to Solve the MTVRP}

The exact method we propose consists of seven main steps and can be described as follows.

1. Solving relaxation $\overline{\text { LRF1 }}$ with procedures $\mathbf{H}^{\mathbf{1}}$ and $\mathbf{H}^{2}$. Solve relaxation $\overline{L R F 1}$ with SC and WSR3 inequalities, executing, in sequence, procedures $H^{1}$ and $H^{2}$. Let $L B_{2}$ be the cost of the dual solution $\left(\boldsymbol{u}^{\mathbf{2}}, \boldsymbol{v}^{\mathbf{2}}, \boldsymbol{g}^{\mathbf{2}}\right)$ achieved by $H^{2}$ (see $\S \S 5.5 .2$ and 5.5.4).

2. Generating path set $\mathscr{P}^{2}$ and route set $\mathscr{R}^{2}$. Generate path set $\mathscr{P}^{2}$ and set $\mathscr{R}^{2}$ of the routes of reduced cost with respect to $\left(\boldsymbol{u}^{\mathbf{2}}, \boldsymbol{v}^{\mathbf{2}}, \boldsymbol{g}^{\mathbf{2}}\right)$ less than or equal to $z_{U B}-L B_{2}$ (see $§ 5.5 .5$ ). If $\mathscr{P}^{2}$ is not optimal, Stop (the algorithm terminates without providing any optimal solution).

3. Solving relaxation $\overline{\mathbf{L R F} 1}$ with procedure $\mathbf{H}^{3}$. Solve relaxation $\overline{L R F 1}$ with SC, SR3, SR5, and WT inequalities with procedure $H^{3}$ (see §5.5.6). Let $L B_{3}$ be the lower bound corresponding to the dual solution $\left(\boldsymbol{u}^{\mathbf{3}}, \boldsymbol{v}^{\mathbf{3}}, \boldsymbol{g}^{\mathbf{3}}, \boldsymbol{h}^{\mathbf{3}}\right)$ of $\overline{L R F 1}$ achieved by $H^{3}$.

4. Generating the route set $\mathscr{R}^{3}$. Generate the largest set $\mathscr{R}^{3}$ of the routes of reduced cost with respect to $\left(\boldsymbol{u}^{\mathbf{3}}, \boldsymbol{v}^{\mathbf{3}}, \boldsymbol{g}^{\mathbf{3}}, \boldsymbol{h}^{\mathbf{3}}\right)$ less than or equal to $z_{U B}-L B_{3}$ and such that $\left|\mathscr{R}^{3}\right| \leq \Delta(\mathscr{R})$ (see $\S 5.5 .7$ ). If $\left|\mathscr{R}^{3}\right|<\Delta(\mathscr{R}), \mathscr{R}^{3}$ contains the routes of any optimal solution of cost less than or equal to $z_{U B}$ and $\mathscr{R}^{3}$ is defined optimal. Otherwise, $\mathscr{R}^{3}$ is defined not-optimal. 
5. Solving the reduced problem F1. If $m=1$, or if $m>1, \mathscr{R}^{3}$ is optimal, and $\left|\mathscr{R}^{3}\right| \leq 5,000$, then find an optimal MTVRP solution by solving, with an IP solver, problem $F 1$ by replacing the route set $\mathscr{R}$ with the route set $\mathscr{R}^{3}$, and Stop.

6. Solving relaxation $\overline{\mathbf{L F 2}}$ with procedure $\mathbf{H}^{4}$. If $m>1$, and either $\mathscr{R}^{3}$ is not optimal or $\left|\mathscr{R}^{3}\right|>5,000$, then solve $\overline{L F 2}$ with SC, SR3, and SR5 inequalities with $H^{4}$ (see $\S 5.6$ ). Let $L B_{4}$ be the lower bound corresponding to an optimal dual solution $\left(\boldsymbol{w}^{\mathbf{4}}, \boldsymbol{v}^{\mathbf{4}}, \boldsymbol{g}^{\mathbf{4}}\right)$ of $\overline{L F 2}$ obtained by $H^{4}$. If the optimal primal solution $\boldsymbol{y}$ of $\overline{L F 2}$ is integer, Stop.

7. Solving the reduced problem F2. Call GenSched to generate the largest set of schedules $\mathscr{H}^{4} \subseteq \mathscr{H}$, by setting $(\tilde{\boldsymbol{w}}, \tilde{\boldsymbol{v}}, \tilde{\boldsymbol{g}})=\left(\boldsymbol{w}^{\mathbf{4}}, \boldsymbol{v}^{\mathbf{4}}, \boldsymbol{g}^{\mathbf{4}}\right), \gamma=z_{U B}-L B_{4}$, $\Delta(\mathscr{D})=10^{6}$.

If $\left|\mathscr{H}^{4}\right| \geq \Delta(\mathscr{D})$, as there is no guarantee that $\mathscr{H}^{4}$ contains all schedules of any optimal MTVRP solution, the algorithm terminates prematurely without providing any optimal solution.

Otherwise (i.e., if $\left|\mathscr{H}^{4}\right|<\Delta(\mathscr{D})$ ) compute an optimal MTVRP solution by solving, with an IP solver, the integer problem $F 2$ obtained by replacing the schedule set $\mathscr{H}$ with the subset $\mathscr{H}^{4}$ and by adding all the valid inequalities saturated at the end of $H^{4}$.

\subsection{Computational Results}

This section reports on the computational results of the exact method described in §5.8. All algorithms were coded in $\mathrm{C}$ and compiled with Visual Studio 2008. CPLEX 12.1 was used as the LP solver in $H^{3}$ and $H^{4}$ and as the IP solver in Steps 5 and 7 of the exact method. All tests were performed on a Sony Vaio P8400 laptop (Intel Core 2 Duo@2.26 GHz with 4 GB of RAM).

The exact algorithm was tested on a subset of the benchmark instances proposed in Taillard et al. [1996], which were used to test all heuristic algorithms in the literature. These instances were generated by starting from the graphs, demands and vehicle capacities of five CVRP problems (namely, CMT-1, CMT-2, CMT-3, CMT-11, and CMT-12) proposed in Christofides et al. [1979] and by varying the number of vehicles, $m$. For each problem and each value of $m$, two instances with different values of $T$ were generated: in the first instance, $T=\left[\frac{1.05 z_{R T}}{m}\right]$, and in the second one, $T=\left[\frac{1.10 z_{R T}}{m}\right]$, where $[x]$ is the integer value nearest to $x$ and $z_{R T}$ is the CVRP solution cost obtained by Rochat and Taillard [1995]. All instances feature $x-y$ coordinates. The travel costs coincide with the travel times and are real values computed as Euclidean distances between the vertices. For an accurate description of the test instances see Taillard et al. [1996]. 
TABLE 5.1: Upper Bounds on the Test Instances

\begin{tabular}{|c|c|c|c|c|c|c|c|c|c|c|c|c|c|c|}
\hline Inst & $m$ & $T$ & $z_{U B}$ & $T_{U B}$ & Inst & $m$ & $T$ & $z_{U B}$ & $T_{U B}$ & Inst & $m$ & $T$ & $z_{U B}$ & $T_{U B}$ \\
\hline \multirow[t]{6}{*}{ CMT-1 } & 1 & 551 & 524.61 & 27 & \multirow[t]{12}{*}{ CMT-3 } & 1 & 867 & 826.14 & 106 & \multirow[t]{22}{*}{ CMT-12 } & 1 & 861 & 819.56 & 93 \\
\hline & 2 & 275 & 533.00 & 27 & & 2 & 434 & 826.14 & 105 & & 2 & 430 & 819.56 & 95 \\
\hline & 1 & 577 & 524.61 & 27 & & 3 & 289 & 826.14 & 107 & & 3 & 287 & 819.56 & 95 \\
\hline & 2 & 289 & 529.85 & 28 & & 4 & 217 & 828.73 & 105 & & 4 & 215 & 819.56 & 95 \\
\hline & 3 & 192 & 552.68 & 30 & & 5 & 173 & 851.47 & 107 & & 5 & 172 & 845.37 & 95 \\
\hline & 4 & 144 & 546.29 & 28 & & 6 & 145 & 839.90 & 172 & & 1 & 902 & 819.56 & 95 \\
\hline \multirow[t]{16}{*}{ CMT-2 } & 1 & 877 & 835.26 & 49 & & 1 & 909 & 826.14 & 106 & & 2 & 451 & 819.56 & 93 \\
\hline & 2 & 439 & 835.26 & 49 & & 2 & 454 & 826.14 & 106 & & 3 & 301 & 819.56 & 93 \\
\hline & 3 & 292 & 835.26 & 49 & & 3 & 303 & 826.14 & 105 & & 4 & 225 & 819.56 & 96 \\
\hline & 4 & 219 & 835.26 & 50 & & 4 & 227 & 826.14 & 107 & & 5 & 180 & 824.78 & 96 \\
\hline & 5 & 175 & 835.80 & 50 & & 5 & 182 & 833.15 & 109 & & 6 & 150 & 825.36 & 96 \\
\hline & 6 & 146 & 857.00 & 111 & & 6 & 151 & 842.21 & 109 & & & & & \\
\hline & 1 & 919 & 835.26 & 49 & \multirow[t]{10}{*}{ CMT-11 } & 1 & 1,094 & $1,042.11$ & 156 & & & & & \\
\hline & 2 & 459 & 835.26 & 49 & & 2 & 547 & $1,042.11$ & 161 & & & & & \\
\hline & 3 & 306 & 835.26 & 50 & & 3 & 365 & $1,042.11$ & 160 & & & & & \\
\hline & 4 & 230 & 835.26 & 50 & & 4 & 274 & $1,085.92$ & 210 & & & & & \\
\hline & 5 & 184 & 835.26 & 51 & & 5 & 219 & $1,042.11$ & 161 & & & & & \\
\hline & 6 & 153 & 839.22 & 51 & & 1 & 1,146 & $1,042.11$ & 160 & & & & & \\
\hline & 7 & 131 & 872.64 & 51 & & 2 & 573 & $1,042.11$ & 159 & & & & & \\
\hline & & & & & & 3 & 382 & $1,042.11$ & 160 & & & & & \\
\hline & & & & & & 4 & 287 & $1,042.11$ & 161 & & & & & \\
\hline & & & & & & 5 & 229 & $1,042.11$ & 164 & & & & & \\
\hline
\end{tabular}

The exact method described in $\S 5.8$ requires an upper bound $z_{U B}$ (see Steps 2, 4 and 7). In our experiments, such upper bound was set equal to the best of three upper bounds: (a) the upper bound computed by the heuristic of Taillard et al. [1996] (sent us by private communication Taillard [2009]), (b) the upper bound reported in Olivera and Viera [2007], and (c) the cost of the CVRP solution found by the heuristic of Mester and Bräysy [2005] if the routes of such solution can be arranged so as to obtain a feasible MTVRP solution.

For each instance, Table 5.1 reports the number of vehicles $(m)$, the maximum driving time $(T)$, the upper bound $\left(z_{U B}\right)$, and the estimated computing time for computing $z_{U B}\left(T_{U B}\right)$ on our machine. The estimated computing time $T_{U B}$ is the sum of the computing times of the algorithms of Taillard et al. [1996], Olivera and Viera [2007], and Mester and Bräysy [2005] normalized to our machine by considering that, according to http://www.cpubenchmark.net/cpu_list.php, our machine is $\approx 100$ times faster than the $100 \mathrm{MHz}$ machine used by Taillard et al. [1996], 4 times faster than the 1.8 GHz AMD Athlon XP 2200+ used by Olivera and Viera [2007], and 6.3 times faster than the Pentium $42 \mathrm{GHz}$ used by Mester and Bräysy [2005].

We did not test our algorithm on the four instances for which no feasible solution is known (namely, instances CMT- 1 with $m=3$ and $T=184$, CMT- 1 with $m=4$ and $T=138$, CMT-2 with $m=7$ and $T=125$, and CMT-12 with $m=6$ and $T=143$ ) because the exact algorithm described in $\S 5.8$ would run out of memory at Step 2 while generating the path set $\mathscr{P}^{2}$. 
TABle 5.2: Computational Results on CMT-1, CMT-2 and CMT-3 instances

\begin{tabular}{|c|c|c|c|c|c|c|c|c|c|c|c|c|c|c|c|c|c|c|c|c|}
\hline \multirow[b]{2}{*}{ Inst } & \multirow[b]{2}{*}{$m$} & \multirow[b]{2}{*}{$T$} & \multirow[b]{2}{*}{$z_{U B}$} & \multirow[b]{2}{*}{$z^{*}$} & \multicolumn{8}{|c|}{ Formulation $F 1$} & \multicolumn{2}{|c|}{ Solving $F 1$} & \multicolumn{4}{|c|}{ Formulation $F 2$} & \multicolumn{2}{|c|}{ Solving $F 2$} \\
\hline & & & & & $\% L B_{1}$ & $\% L B_{2}$ & $\mathscr{P}^{2}$ & $\mathscr{R}^{2}$ & $L B_{3}$ & $\% L B_{3}$ & $T_{3}$ & $\mathscr{R}^{3}$ & $T_{I P}$ & $T_{T O T}$ & $\mathrm{LB}_{4}$ & $\% \mathrm{LB}_{4}$ & $T_{4}$ & $\mathscr{H}^{4}$ & $T_{I P}$ & $T_{T O T}$ \\
\hline \multirow{6}{*}{$\begin{array}{l}\text { CMT-1 } \\
\mathrm{n}=50\end{array}$} & 1 & 551 & 524.61 & 524.61 & 99.6 & 99.8 & 2,549 & 329 & 524.61 & 100.0 & 9.4 & 47 & 0.1 & 9.5 & & & & & & \\
\hline & 2 & 275 & 533.00 & 533.00 & 98.1 & 98.3 & 142,645 & 95,763 & 532.22 & 99.9 & 36.3 & 509 & 0.9 & 37.2 & & & & & & \\
\hline & 1 & 577 & 524.61 & 524.61 & 99.6 & 99.8 & 1,922 & 329 & 524.61 & 100.0 & 10.3 & 62 & 0.5 & 10.8 & & & & & & \\
\hline & 2 & 289 & 529.85 & 529.85 & 98.7 & 98.9 & 35,676 & 16,669 & 527.68 & 99.6 & 15.0 & 806 & 1.9 & 16.9 & & & & & & \\
\hline & 3 & 192 & 552.68 & & 94.6 & 94.8 & m.o. & - & & & 34.8 & & & & & & & & & \\
\hline & 4 & 144 & 546.29 & 546.29 & 95.7 & 96.0 & $2,365,662$ & $3,941,493$ & 543.50 & 99.5 & 588.8 & 1,268 & 15.5 & 603.3 & & & & & & \\
\hline \multirow{13}{*}{$\begin{array}{l}\text { CMT-2 } \\
\mathrm{n}=75\end{array}$} & 1 & 877 & 835.26 & 835.26 & 98.5 & 98.9 & 138,112 & 114,078 & 832.78 & 99.7 & 205.9 & 3,117 & 10.6 & 216.5 & & & & & & \\
\hline & 2 & 439 & 835.26 & 835.26 & 98.5 & 98.9 & 138,112 & 114,078 & 832.78 & 99.7 & 205.9 & 3,117 & 16.5 & 222.4 & & & & & & \\
\hline & 3 & 292 & 835.26 & 835.26 & 98.5 & 98.9 & 138,112 & 114,078 & 832.78 & 99.7 & 205.9 & 3,117 & 21.0 & 226.9 & & & & & & \\
\hline & 4 & 219 & 835.26 & 835.26 & 98.5 & 98.9 & 138,112 & 114,078 & 832.78 & 99.7 & 205.9 & 3,117 & 27.7 & 233.6 & & & & & & \\
\hline & 5 & 175 & 835.80 & 835.80 & 98.5 & 98.8 & 170,128 & 148,826 & 832.77 & 99.6 & 188.8 & 4,746 & 336.0 & 524.8 & & & & & & \\
\hline & 6 & 146 & 857.00 & & 96.0 & 96.4 & m.o. & & & & 48.3 & & & & & & & & & \\
\hline & 1 & 919 & 835.26 & 835.26 & 98.5 & 98.9 & 138,112 & 114,078 & 832.78 & 99.7 & 205.9 & 3,117 & 10.6 & 216.5 & & & & & & \\
\hline & 2 & 459 & 835.26 & 835.26 & 98.5 & 98.9 & 138,112 & 114,078 & 832.78 & 99.7 & 205.9 & 3,117 & 16.5 & 222.4 & & & & & & \\
\hline & 3 & 306 & 835.26 & 835.26 & 98.5 & 98.9 & 138,112 & 114,078 & 832.78 & 99.7 & 205.9 & 3,117 & 21.4 & 227.3 & & & & & & \\
\hline & 4 & 230 & 835.26 & 835.26 & 98.5 & 98.9 & 138,112 & 114,078 & 832.78 & 99.7 & 205.9 & 3,117 & 28.6 & 234.5 & & & & & & \\
\hline & 5 & 184 & 835.26 & 835.26 & 98.5 & 98.9 & 138,112 & 114,078 & 832.78 & 99.7 & 205.9 & 3,117 & 54.2 & 260.1 & & & & & & \\
\hline & 6 & 153 & 839.22 & 839.22 & 98.1 & 98.4 & 472,756 & 551,114 & 832.79 & 99.2 & 221.4 & 49,511 & $\Rightarrow$ & & 834.12 & 99.4 & 374.2 & 343,626 & $4,209.1$ & $4,583.3$ \\
\hline & 7 & 131 & 872.64 & & 94.3 & 94.7 & m.o. & - & - & - & 47.6 & & & & & & & & & \\
\hline \multirow{12}{*}{$\begin{array}{l}\text { CMT-3 } \\
\mathrm{n}=100\end{array}$} & 1 & 867 & 826.14 & 826.14 & 98.7 & 99.0 & $5,248,271$ & $>5 \cdot 10^{6}$ & 826.14 & 100.0 & $2,766.7$ & 1,471 & 0.1 & $2,766.8$ & & & & & & \\
\hline & 2 & 434 & 826.14 & 826.14 & 98.7 & 99.0 & $5,248,271$ & $>5 \cdot 10^{6}$ & 826.14 & 100.0 & $2,766.7$ & 1,471 & 15.0 & $2,781.7$ & & & & & & \\
\hline & 3 & 289 & 826.14 & 826.14 & 98.7 & 99.0 & $5,248,271$ & $>5 \cdot 10^{6}$ & 826.14 & 100.0 & $2,766.7$ & 1,471 & 27.2 & $2,793.9$ & & & & & & \\
\hline & 4 & 217 & 828.73 & & 98.4 & 98.7 & m.o. & - & - & - & 96.2 & & & & & & & & & \\
\hline & 5 & 173 & ${ }^{A} 851.47$ & & 97.6 & 97.9 & m.o. & - & - & - & 95.7 & & & & & & & & & \\
\hline & 6 & 145 & B 839.90 & & 97.5 & 97.9 & m.o. & - & - & - & 93.4 & & & & & & & & & \\
\hline & 1 & 909 & 826.14 & 826.14 & 98.7 & 99.0 & $5,248,271$ & $>5 \cdot 10^{6}$ & 826.14 & 100.0 & $2,766.7$ & 1,471 & 0.1 & $2,766.8$ & & & & & & \\
\hline & 2 & 454 & 826.14 & 826.14 & 98.7 & 99.0 & $5,248,271$ & $>5 \cdot 10^{6}$ & 826.14 & 100.0 & $2,766.7$ & 1,471 & 15.3 & $2,782.0$ & & & & & & \\
\hline & 3 & 303 & 826.14 & 826.14 & 98.7 & 99.0 & $5,248,271$ & $>5 \cdot 10^{6}$ & 826.14 & 100.0 & $2,766.7$ & 1,471 & 28.5 & $2,795.2$ & & & & & & \\
\hline & 4 & 227 & 826.14 & 826.14 & 98.7 & 99.0 & $5,248,271$ & $>5 \cdot 10^{6}$ & 826.14 & 100.0 & $2,766.7$ & 1,471 & 46.6 & $2,813.3$ & & & & & & \\
\hline & 5 & 182 & 833.15 & & 97.9 & 98.2 & m.o. & - & - & - & 94.1 & & & & & & & & & \\
\hline & 6 & 151 & ${ }^{C} 842.21$ & & 97.5 & 97.8 & m.o. & - & - & - & 88.0 & & & & & & & & & \\
\hline
\end{tabular}

$B$ Best known upper bound 836.21 from Olivera [2005] 
TABLE 5.3: Computational Results on CMT-11 and CMT-12 instances

\begin{tabular}{|c|c|c|c|c|c|c|c|c|c|c|c|c|c|c|c|c|c|c|c|c|}
\hline \multirow[b]{2}{*}{ Inst } & \multirow[b]{2}{*}{$m$} & \multirow[b]{2}{*}{$T$} & \multirow[b]{2}{*}{$z_{U B}$} & \multirow[b]{2}{*}{$z^{*}$} & \multicolumn{8}{|c|}{ Formulation $F 1$} & \multicolumn{2}{|c|}{ Solving $F 1$} & \multicolumn{4}{|c|}{ Formulation $F 2$} & \multicolumn{2}{|c|}{ Solving $F 2$} \\
\hline & & & & & $\% L B_{1}$ & $\% L B_{2}$ & $\mathscr{P}^{2}$ & $\mathscr{R}^{2}$ & $L B_{3}$ & $\% L B_{3}$ & $T_{3}$ & $\mathscr{R}^{3}$ & $\overline{T_{I P}}$ & $\overline{T_{T O T}}$ & $L B_{4}$ & $\% L B_{4}$ & $T_{4}$ & $\mathscr{H}^{4}$ & $T_{I P}$ & $T_{T O T}$ \\
\hline CMT-11 & 1 & 1,094 & $1,042.11$ & $1,042.11$ & 99.6 & 99.9 & 185,303 & 4,705 & $1,042.11$ & 100.0 & 632.1 & 83 & 0.2 & 632.3 & & & & & & \\
\hline \multirow[t]{9}{*}{$\mathrm{n}=120$} & 2 & 547 & $1,042.11$ & $1,042.11$ & 99.6 & 99.9 & 185,303 & 4,705 & $1,042.11$ & 100.0 & 632.1 & 83 & 0.2 & 632.3 & & & & & & \\
\hline & 3 & 365 & $1,042.11$ & $1,042.11$ & 99.6 & 99.9 & 185,303 & 4,705 & $1,042.11$ & 100.0 & 632.1 & 83 & 0.2 & 632.3 & & & & & & \\
\hline & 4 & 274 & ${ }^{A} 1,085.92$ & & 96.3 & 96.6 & m.o. & - & - & - & 776.1 & & & & & & & & & \\
\hline & 5 & 219 & $1,042.11$ & $1,042.11$ & 99.6 & 99.9 & 182,530 & 5,102 & $1,042.11$ & 100.0 & 620.0 & 105 & 0.2 & 620.2 & & & & & & \\
\hline & 1 & 1,146 & $1,042.11$ & $1,042.11$ & 99.6 & 99.9 & 185,303 & 4,705 & $1,042.11$ & 100.0 & 632.1 & 83 & 0.2 & 632.3 & & & & & & \\
\hline & 2 & 573 & $1,042.11$ & $1,042.11$ & 99.6 & 99.9 & 185,303 & 4,705 & $1,042.11$ & 100.0 & 632.1 & 83 & 0.2 & 632.3 & & & & & & \\
\hline & 3 & 382 & $1,042.11$ & $1,042.11$ & 99.6 & 99.9 & 185,303 & 4,705 & $1,042.11$ & 100.0 & 632.1 & 83 & 0.2 & 632.3 & & & & & & \\
\hline & 4 & 287 & $1,042.11$ & $1,042.11$ & 99.6 & 99.9 & 185,303 & 4,705 & $1,042.11$ & 100.0 & 631.0 & 83 & 0.2 & 631.2 & & & & & & \\
\hline & 5 & 229 & $1,042.11$ & $1,042.11$ & 99.6 & 99.9 & 185,303 & 4,705 & $1,042.11$ & 100.0 & 619.8 & 83 & 0.2 & 620.0 & & & & & & \\
\hline CMT-12 & 1 & 861 & 819.56 & 819.56 & 99.7 & 100.0 & 49,368 & 71 & 819.56 & 100.0 & 39.9 & 24 & 0.3 & 40.2 & & & & & & \\
\hline \multirow[t]{10}{*}{$\mathrm{n}=100$} & 2 & 430 & 819.56 & 819.56 & 99.7 & 100.0 & 46,578 & 60 & 819.56 & 100.0 & 44.7 & 21 & 0.3 & 45.0 & & & & & & \\
\hline & 3 & 287 & 819.56 & 819.56 & 99.7 & 100.0 & 43,219 & 95 & 819.56 & 100.0 & 43.0 & 23 & 0.2 & 43.2 & & & & & & \\
\hline & 4 & 215 & 819.56 & 819.56 & 99.7 & 100.0 & 46,578 & 60 & 819.56 & 100.0 & 45.0 & 21 & 0.2 & 45.2 & & & & & & \\
\hline & 5 & 172 & 845.37 & & 96.6 & 96.9 & m.o. & - & - & - & 16.6 & & & & & & & & & \\
\hline & 1 & 902 & 819.56 & 819.56 & 99.7 & 100.0 & 49,368 & 71 & 819.56 & 100.0 & 39.9 & 24 & 0.3 & 40.2 & & & & & & \\
\hline & 2 & 451 & 819.56 & 819.56 & 99.7 & 100.0 & 46,578 & 60 & 819.56 & 100.0 & 44.7 & 21 & 0.3 & 45.0 & & & & & & \\
\hline & 3 & 301 & 819.56 & 819.56 & 99.7 & 100.0 & 43,219 & 95 & 819.56 & 100.0 & 43.0 & 23 & 0.3 & 43.3 & & & & & & \\
\hline & 4 & 225 & 819.56 & 819.56 & 99.7 & 100.0 & 46,578 & 60 & 819.56 & 100.0 & 45.0 & 21 & 0.5 & 45.5 & & & & & & \\
\hline & 5 & 180 & 824.78 & 824.78 & 99.0 & 99.4 & $1,857,419$ & 818,631 & 819.56 & 99.4 & 314.6 & 746,542 & $\Rightarrow$ & & 823.69 & 99.9 & $2,290.3$ & 274,857 & 77.6 & $2,367.9$ \\
\hline & 6 & 150 & 825.36 & 823.14 & 99.2 & 99.6 & $2,724,943$ & $1,717,379$ & 819.56 & 99.6 & 402.5 & $1,520,016$ & $\Rightarrow$ & & 823.14 & 100.0 & $1,058.2$ & 3,819 & 0.2 & $1,058.4$ \\
\hline
\end{tabular}


Tables 5.2 and 5.3 report detailed computational results. For each instance, the columns report the following information: instance name, Inst; number of vehicles, $m$; maximum driving time, $T$; initial upper bound, $z_{U B}$; optimal solution cost, $z^{*}$; percentage lower bound, $\% L B_{k}(k=1, \ldots, 4)$, achieved by bounding procedure $H^{k}$; lower bounds, $L B_{3}$ and $L B_{4}$, achieved by bounding procedures $H^{3}$ and $H^{4}$, respectively; cardinality of the sets $\mathscr{P}^{2}, \mathscr{R}^{2}, \mathscr{R}^{3}, \mathscr{H}^{4}$; global time spent, $T_{3}$, to execute $H^{1}, H^{2}$, and $H^{3}$; time spent, $T_{4}$, by $H^{4}$ (including $T_{3}$ ); time spent, $T_{I P}$, by CPLEX to solve $F 1$ or $F 2$; total computing time, $T_{T O T}$. In column $\mathscr{P}^{2}$, "m.o." means that the exact method terminated prematurely at Step 2 because $\mathscr{P}^{2}$ is not optimal (i.e., $\left.\left|\mathscr{P}^{2}\right|>\Delta(\mathscr{P})=10^{7}\right)$. In this case, $T_{3}$ indicates the time spent by $H^{1}$ and $H^{2}$ plus

the computing time for generating $\mathscr{P}^{2}$.

Table 5.2 and 5.3 show that the proposed method could solve 42 out of 52 test instances. All but one of the instances were solved within one hour of computing time. Thirty-five of the 42 instances solved to optimality have the optimal solution cost equal to the cost of the corresponding CVRP instance. The other seven instances have optimal solution cost slightly greater than the cost of the corresponding CVRP instance.

The lower bounds proposed seem to be effective. The lower bounds achieved by $H^{1}$ and $H^{2}$ are, on average, $98.6 \%$ and $98.9 \%$ with respect to the best known solution cost, respectively. Bounding procedure $H^{3}$ closed the gap of 26 instances. Only three instances were solved by using formulation $F 2$. In all such cases, lower bound $L B_{4}$ is strictly greater than $L B_{3}$, requiring the generation of few schedules (set $\mathscr{H}^{4}$ ) in Step 7 with respect to the routes generated at Step 4 (set $\left.\mathscr{R}^{3}\right)$.

For the sake of fairness, we have to say that the performance of the proposed algorithm strongly depends on the initial upper bound, $z_{U B}$, used at Steps 2, 4 and 7. For example, by increasing such upper bound by $1 \%$, the exact method, as it is, could not solve any of the instances of the classes CMT-2, CMT-3 and CMT-11, and it could solve only 12 out of 17 instances of the classes CMT-1 and CMT-12. Anyway, as shown in Table 5.1, high-quality upper bounds can be computed in a few minutes by running the heuristic algorithms of Taillard et al. [1996], Olivera and Viera [2007], and Mester and Bräysy [2005].

\subsection{Conclusions}

In this chapter, we describe an exact method to solve the MTVRP based on two set partitioning-like formulations. We describe four different bounding procedures, based on the linear relaxations of both formulations, enforced by valid inequalities, that are embedded into an exact solution method. The computational results show that the proposed exact algorithm can solve, to optimality, 42 out of 52 benchmark instances, involving up to 120 customers, used in the literature by the heuristic algorithms. 


\section{Chapter 6}

\section{Two-Echelon Capacitated Vehicle Routing Problem}

1

In the two-echelon capacitated vehicle routing problem (2E-CVRP), the delivery to customers from a depot uses intermediate depots, called satellites. The 2E-CVRP involves two levels of routing problems. The first level requires a design of the routes for a vehicle fleet located at the depot to transport the customer demands to a subset of the satellites. The second level concerns the routing of a vehicle fleet located at the satellites to supply all customers from the satellites supplied from the depot. The objective is to minimize the sum of routing and handling costs. This chapter describes a new mathematical formulation of the $2 \mathrm{E}$-CVRP used to derive valid lower bounds and an exact method. Computational results on benchmark instances show that the new exact algorithm outperforms the state-of-the-art exact methods from the literature.

\subsection{Introduction}

The two-echelon capacitated vehicle routing problem (2E-CVRP) is a two-level distribution system where the deliveries to customers from a depot are managed through intermediate capacitated depots, called satellites. The first level consists of vehicle routes that start and end at the depot and deliver the customer demands to a subset of satellites. In the $2 \mathrm{E}-\mathrm{CVRP}$ we consider, a satellite has a limited capacity and can be serviced by more than one $1^{\text {st }}$-level route. The second level consists of vehicle routes that start and end at the same satellite and supply all customers. A homogeneous vehicle fleet is used at each level. The $1^{\text {st }}$-level vehicles are located at the depot and supply the satellites only. The $2^{\text {nd }}$-level vehicles have a capacity smaller than that

\footnotetext{
${ }^{1}$ This chapter is based on Baldacci et al. [2012b]
} 
of the $1^{\text {st }}$-level vehicles and supply the customers from the satellites. The operations of unloading $1^{\text {st }}$-level vehicles and loading $2^{n d}$-level vehicles at the satellites imply a handling cost, which we assume to be proportional to the quantity loaded/unloaded.

The $2 \mathrm{E}$-CVRP aims to find two sets of $1^{\text {st }}$ and $2^{\text {nd }}$-level routes such that each customer is visited exactly once by a $2^{n d}$-level route and the total routing and handling cost is minimized.

\subsection{Literature Review}

The 2E-CVRP has become a relevant distribution system for supplying customers located in large cities. Because many municipalities impose legal restrictions to keep large vehicles out of city centers, distribution companies create suburban platforms (satellites) where they transport goods with large vehicles. Then, small vehicles service downtown customers from the satellites.

Nonetheless, only recently the $2 \mathrm{E}-\mathrm{CVRP}$ has received some attention in the literature. Gonzales Feliu et al. [2007b] described a commodity flow formulation and an exact branch-and-cut algorithm that solved instances with up to 32 customers and 2 satellites. This algorithm was improved by Perboli et al. [2010] and Perboli et al. [2011] by adding valid inequalities. Perboli et al. [2011] reported optimal solutions for instances with up to 32 customers and 2 satellites, but their model has been shown by Jepsen et al. [2011] not to be correct on instances with 3 or more satellites. Jepsen et al. [2011] extended the problem considered by Gonzales Feliu et al. [2007b] and Perboli et al. [2011] by introducing fixed costs for the routes of both levels and satellite capacities; they described an exact branch-and-cut algorithm, based on the new formulation and new valid inequalities, that outperforms the method of Perboli et al. [2011]. Heuristic methods have been proposed by Crainic et al. [2008], Perboli et al. [2011], Crainic et al. [2011] and Hemmelmayr et al. [2011]. Variants of the 2E-CVRP were considered by Chao [2002], Tan et al. [2006] and Nguyen et al. [2010].

The 2E-CVRP considered in this paper corresponds to the 2E-CVRP considered by Jepsen et al. [2011] and generalizes the capacitated location routing problem (LRP). The LRP consists of opening one or more depots, on a given set of a-priori defined depot locations, and designing, for each open depot, a number of routes to supply customers. A fixed cost and a capacity are associated with each depot. The objective is to minimize the sum of the fixed costs for opening the depots and the routing cost. Exact algorithms for the LRP were presented by Laporte et al. [1986], Akca et al. [2009], Belenguer et al. [2011], and Baldacci et al. [2011c].

In this chapter, we introduce a new mathematical formulation of the 2E-CVRP that is used to derive both integer and continuous relaxations. We present a new bounding procedure based on dynamic programming (DP), a dual-ascent method, and an exact 
algorithm that decomposes the 2E-CVRP into a limited set of multi-depot capacitated vehicle routing problems (MDCVRP) with side constraints. Extensive computational results on instances from the literature and on new instances show that the proposed method outperforms previous exact algorithms, both for the quality of the lower bounds achieved and the number and the size of the instances solved.

\subsection{Problem Description and Mathematical Formulation}

An undirected graph $G=(N, E)$ is given, where the vertex set $N$ is partitioned as $N=\{0\} \cup N_{S} \cup N_{C}$. Vertex 0 represents the depot, the set $N_{S}=\left\{1,2, \ldots, n_{s}\right\}$ represents $n_{s}$ satellites, and the set $N_{C}=\left\{n_{s}+1, \ldots, n_{s}+n_{c}\right\}$ represents $n_{c}$ customers. The edge set $E$ is defined as $E=\left\{\{0, j\}: j \in N_{S}\right\} \cup\left\{\{i, j\}: i, j \in N_{S} \cup N_{C}, i<j\right\}$. A travel cost $d_{i j}$ is associated with each edge $\{i, j\} \in E$. We assume that matrix $d_{i j}$ satisfies the triangle inequality. Each customer $i \in N_{C}$ requires $q_{i}$ units of goods from depot 0 . We denote with $q_{t o t}=\sum_{i \in N_{C}} q_{i}$ the sum of the customer demands.

A fleet of $m^{1}$ identical vehicles of capacity $Q_{1}$ are located at depot 0 and are used to transport goods to satellites. If used, a $1^{\text {st }}$-level vehicle incurs a fixed cost $U_{1}$ and performs a route passing through the depot 0 and a subset of satellites. The cost of a $1^{s t}$-level route is the sum of the costs of the traversed edges plus the fixed cost $U_{1}$. Each satellite $k \in N_{S}$ can be visited by more than one $1^{\text {st }}$-level route and has a capacity $B_{k}$ that limits the total customer demand that can be delivered to it by the $1^{\text {st }}$-level routes. Moreover, a fleet of $m_{k}$ identical vehicles of capacity $Q_{2}<Q_{1}$ are available at satellite $k \in N_{S}$ for servicing the customers. Nevertheless, at most $m^{2} \leq \sum_{k \in N_{S}} m_{k}$ $2^{\text {nd }}$-level vehicles can be globally used. If used, a $2^{\text {nd }}$-level vehicle incurs a fixed cost $U_{2}$ and performs a route, that is a simple cycle in $G$ passing through a satellite and a subset of customers and such that the total demand of the visited customers does not exceed the vehicle capacity $Q_{2}$. The cost of a $2^{\text {nd }}$-level route is the sum of the traversed edges plus the fixed cost $U_{2}$. The handling cost at satellite $k \in N_{S}$ is given by $H_{k}$ times the quantity delivered to satellite $k$.

The problem asks to design the vehicle routes of both levels so that each customer is visited exactly once, the quantity delivered to customers from each satellite is equal to the quantity received from the depot, and the sum of the routing and handling costs is minimized.

The 2E-CVRP contains as a special case the LRP. The LRP is defined on an undirected graph $G^{\prime}=\left(N^{\prime}, E^{\prime}\right)$, where $N^{\prime}$ is partitioned as $N^{\prime}=L \cup V$, where $L$ represents possible depot locations and $V$ a set of customers. A travel cost $d_{i j}$ is associated with each edge $\{i, j\} \in E^{\prime}$. A fixed cost $C_{k}$ and a capacity $B_{k}$ are associated with each depot location $k \in L$. Each customer $i \in V$ has associated a nonnegative demand $q_{i}$. An unlimited fleet of identical vehicles of capacity $Q$ are available at the depots to supply 
the customers. If used, a vehicle incurs a fixed cost $U$ and performs a route passing through one of the depot locations and such that the total demand of the visited customers is at most $Q$. The cost of a route is the sum of the costs of the traversed edges plus the fixed cost $U$. The LRP consists of opening a set of depots and designing a set of routes for each open depot so that the total load of the routes operated from a depot $k \in L$ does not exceed its capacity $B_{k}$ and each customer is visited by exactly one route. The objective is to minimize the sum of the cost of open depots and the costs of the routes.

Any LRP instance can be converted into an equivalent 2E-CVRP instance as follows.

(a) Define graph $G=(N, E)$ by setting $N_{S}=L, N_{C}=V$, and define the edge costs $d_{0 k}=\frac{1}{2} C_{k}, k \in N_{S}$, and $d_{k j}=\infty, k, j \in N_{S}, k<j$.

(b) Define the $1^{s t}$-level vehicle fleet by setting $m^{1}=\left|N_{S}\right|, Q_{1}=\infty$, and $U_{1}=0$;

(c) Define the $2^{n d}$-level vehicle fleet by setting $m^{2}=\left|N_{C}\right|, U_{2}=U, Q_{2}=Q$, and $m_{k}=\left|N_{C}\right|, k \in N_{S}$.

Any optimal solution of the resulting $2 \mathrm{E}-\mathrm{CVRP}$ instance is also an optimal solution of the original LRP instance. Because $d_{k j}=\infty$, for any $k, j \in N_{S}, k<j$, each $1^{\text {st }}$-level vehicle route can only be a single-satellite route $(0, k, 0)$ of cost $C_{k}, k \in N_{S}$. Because $Q_{1}=\infty$, any optimal solution contains at most a single-satellite route for each $k \in N_{S}$ representing the opening of depot $k$.

As for the 2E-CVRP, we assume that the travel cost matrix $\left[d_{i j}\right]$ is modified as follows in order to consider vehicle fixed costs $U_{1}$ and $U_{2}$ : (i) $d_{0 k}=d_{0 k}+\frac{1}{2} U_{1}, k \in N_{S}$, and (ii) $d_{k i}=d_{k i}+\frac{1}{2} U_{2}, k \in N_{S}, i \in N_{C}$.

Let $\mathscr{M}$ be the index set of all $1^{\text {st }}$-level routes, and let $\mathscr{M}_{k} \subseteq \mathscr{M}$ be the subset of $1^{s t}$-level routes serving satellite $k \in N_{S}$. Let $R_{r}$ and $E\left(R_{r}\right)$ be the subset of satellites visited and the subset of edges traversed by $1^{\text {st }}$-level route $r \in \mathscr{M}$, respectively. The cost $g_{r}$ of route $r \in \mathscr{M}$ is $g_{r}=\sum_{\{i, j\} \in E\left(R_{r}\right)} d_{i j}$. We assume that the route set $\mathscr{M}$ contains $\left\lceil\min \left\{m_{k} Q_{2}, q_{t o t}\right\} / Q_{1}\right\rceil$ copies of the single-satellite route $(0, k, 0)$, for each satellite $k \in N_{S}$. Let $w^{\text {min }}$ and $w_{r}^{\max }$ be the minimum and maximum loads of $1^{\text {st }}$. level route $r \in \mathscr{M}$, computed as $w^{\text {min }}=\max \left\{q_{t o t}-\left(m^{1}-1\right) Q_{1}, 0\right\}$ and $w_{r}^{\max }=$ $\min \left\{Q_{1}, q_{t o t}, \sum_{k \in R_{r}} m_{k} Q_{2}\right\}$. Moreover, we denote by $W_{r}=\left\{w \in \mathbb{Z}_{+}: w^{\text {min }} \leq\right.$ $\left.w \leq w_{r}^{\max }\right\}$ the set of possible loads of $1^{\text {st }}$-level route $r \in \mathscr{M}$. Because in real-world applications the number of satellites is small (say, $n_{s} \leq 10$ ), in the following we assume that the set $\mathscr{M}$ is generated by pure enumeration.

Let $\mathscr{R}_{k}$ be the index set of the $2^{n d}$-level routes passing through satellite $k \in N_{S}$, and let $\mathscr{R}_{i k} \subseteq \mathscr{R}_{k}$ be the subset of routes passing through satellite $k \in N_{S}$ and customer $i \in N_{C}$. We indicate with $\mathscr{R}=\cup_{k \in N_{S}} \mathscr{R}_{k}$ the set of all $2^{n d}$-level routes and with $\pi_{\ell}$ the satellite visited by route $\ell \in \mathscr{R}$. Moreover, we indicate with $R_{k \ell}$ and $E\left(R_{k \ell}\right)$ the subset 
of customers visited and the subset of edges traversed by route $\ell \in \mathscr{R}_{k}$, respectively. A load $w_{k \ell}=\sum_{i \in R_{k \ell}} q_{i}$ and a cost $c_{k \ell}=\sum_{\{i, j\} \in E\left(R_{k \ell}\right)} d_{i j}+H_{k} w_{k \ell}$ are associated with route $\ell \in \mathscr{R}_{k}$.

Let $y_{r}$ be a binary variable equal to 1 if and only if route $r \in \mathscr{M}$ is in solution, $x_{k \ell}$ a binary variable equal to 1 if and only if route $\ell \in \mathscr{R}_{k}$ of satellite $k \in N_{S}$ is in solution, and $q_{k r}$ a nonnegative integer variable representing the quantity delivered by $1^{\text {st }}$-level route $r \in \mathscr{M}$ to satellite $k \in R_{r}$ (we assume $q_{k r}=0$, for each satellite $k \in N_{S} \backslash R_{r}$ ). The 2E-CVRP can be formulated as follows

$$
\begin{array}{rlr}
(F) z(F)=\min & \sum_{k \in N_{S}} \sum_{\ell \in \mathscr{R}_{k}} c_{k \ell} x_{k \ell}+\sum_{r \in \mathscr{M}} g_{r} y_{r} & \\
\text { s.t. } & \sum_{k \in N_{S}} \sum_{\ell \in \mathscr{R}_{i k}} x_{k \ell}=1, & \\
& \sum_{\ell \in \mathscr{R}_{k}} x_{k \ell} \leq m_{k}, & \\
& \sum_{k \in N_{S}} \sum_{\ell \in \mathscr{R}_{k}} x_{k \ell} \leq m^{2}, & \\
& \sum_{\ell \in \mathscr{R}_{k}} w_{k \ell} x_{k \ell} \leq B_{k}, & \\
& \sum_{r \in \mathscr{M}} y_{r} \leq m^{1}, & \\
& \sum_{r \in \mathscr{M}_{k}} q_{k r}=\sum_{\ell \in \mathscr{R}_{k}} w_{k \ell} x_{k \ell}, & k \in N_{S}, \\
& \sum_{k \in R_{r}} q_{k r} \leq Q_{1} y_{r}, & \\
& x_{k \ell} \in\{0,1\}, & r \in \mathscr{M}, \\
& y_{r} \in\{0,1\}, & k \in N_{S}, \ell \in \mathscr{R}_{k}, \\
& q_{k r} \in \mathbb{Z}_{+}, & r \in \mathscr{M},
\end{array}
$$

The objective function (6.1) states to minimize the total cost. Constraints (6.2) specify that each customer $i \in N_{C}$ must be visited by exactly one $2^{\text {nd }}$-level route. Constraints (6.3), (6.4), and (6.6) impose the upper bounds on the number of $1^{\text {st }}$ and $2^{n d}$-level routes in solution. Constraints (6.5) impose the satellite capacities. The balance between the quantity delivered by $1^{\text {st }}$-level routes to a satellite and the customer demands supplied from the satellite is imposed by constraints (6.7). Finally, constraints (6.8) impose that the vehicle capacity of the $1^{\text {st }}$-level vehicles is not exceeded.

\subsection{Relaxations of Formulation $F$}

Let $L F$ be the LP-relaxation of formulation $F$, and let $z(L F)$ be its optimal solution cost. Notice that, in any optimal $L F$ solution, $y_{r}$ is equal to $\left(\sum_{k \in R_{r}} q_{k r}\right) / Q_{1}$, for each 
$1^{\text {st }}$-level route $r \in \mathscr{M}$. Thus, the higher the $1^{\text {st }}$-level routing cost, the worse the lower bound $z(L F)$. In the following, we describe an integer relaxation of the 2E-CVRP, called $R F$, that can provide a lower bound better than $z(L F)$.

\subsubsection{Relaxation $R F$}

This relaxation derives from problem $F$ by relaxing, in a Lagrangean fashion, constraints (6.2), (6.3), and (6.4) with penalties $\lambda_{i} \in \mathbb{R}, i \in N_{C}, \mu_{k} \in \mathbb{R}_{-}, k \in N_{S}$, and $\mu_{0} \in \mathbb{R}_{-}$, respectively, and by defining the marginal routing costs $\beta_{i k}$ for servicing customer $i \in N_{C}$ from depot $k \in N_{S}$ as a feasible solution of the following inequalities

$$
\sum_{i \in N_{C}} a_{i k \ell} \beta_{i k} \leq c_{k \ell}-\sum_{i \in N_{C}} a_{i k \ell} \lambda_{i}-\mu_{k}-\mu_{0}, \quad \ell \in \mathscr{R}_{k}, k \in N_{S},
$$

where $a_{i k \ell}$ is the number of times customer $i \in N_{C}$ is visited by route $\ell \in \mathscr{R}_{k}$ of satellite $k \in N_{S}$.

Problem $R F$ involves binary variables $\xi_{i k}$ equal to 1 if customer $i \in N_{C}$ is supplied from satellite $k \in N_{S}$ (0 otherwise) and variables $y_{r}$ and $q_{k r}$, as defined for problem $F$. Relaxation $R F$ is

$$
\begin{array}{rlr}
(R F) \quad z(R F(\boldsymbol{\beta}, \boldsymbol{\lambda}, \boldsymbol{\mu}))=\min & \sum_{k \in N_{S}} \sum_{i \in N_{C}} \beta_{i k} \xi_{i k}+\sum_{r \in \mathscr{M}} g_{r} y_{r}+ & \\
& \sum_{i \in N_{C}} \lambda_{i}+\sum_{k \in N_{S}} m_{k} \mu_{k}+m^{2} \mu_{0} & \\
\text { s.t. } & \sum_{k \in N_{S}} \xi_{i k}=1, & i \in N_{C}, \\
& \sum_{r \in \mathscr{M}_{k}} q_{k r}=\sum_{i \in N_{C}} q_{i} \xi_{i k}, & k \in N_{S}, \\
& \sum_{i \in N_{C}} q_{i} \xi_{i k} \leq B_{k}, & \\
& (6.6),(6.8),(6.10) \text { and }(6.11), & \\
& \xi_{i k} \in\{0,1\}, \quad i \in N_{S},
\end{array}
$$

Theorem 6.1. Value $z(R F(\boldsymbol{\beta}, \boldsymbol{\lambda}, \boldsymbol{\mu}))$ is a valid lower bound on the $2 E-C V R P$ for any solution $\boldsymbol{\beta}$ of inequalities (6.12) and any pair of vectors $\boldsymbol{\lambda} \in \mathbb{R}^{N_{C}}$ and $\boldsymbol{\mu} \in \mathbb{R}_{-}^{N_{S}+1}$.

Proof. Consider an $F$ solution $(\overline{\boldsymbol{x}}, \overline{\boldsymbol{y}}, \overline{\boldsymbol{q}})$ of $\operatorname{cost} \bar{z}(F)$. Let $J_{k}=\left\{\ell \in \mathscr{R}_{k}: \bar{x}_{k \ell}=1\right\}$, $k \in N_{S}$, be the set of $2^{n d}$-level routes and $L=\left\{r \in \mathscr{M}: \bar{y}_{r}=1\right\}$ the set of $1^{\text {st }}$-level routes in solution. We denote by $\bar{V}_{k}=\left\{i \in R_{k \ell}: \ell \in J_{k}\right\}$ the subset of customers serviced from satellite $k \in N_{S}$ and by $\bar{N}_{S}=\left\{k \in R_{r}: r \in L\right\}$ the subset of satellites used in solution. 
Let $z(R F(\boldsymbol{\beta}, \boldsymbol{\lambda}, \boldsymbol{\mu}))$ be the cost of an optimal $R F$ solution for a given set of values $\beta_{i k}$, satisfying inequalities (6.12), and penalty vectors $\boldsymbol{\lambda}$ and $\boldsymbol{\mu}$. Define the reduced cost $\tilde{c}_{k \ell}$ of route $\ell \in \mathscr{R}_{k}, k \in N_{S}$, with respect to $\boldsymbol{\beta}, \boldsymbol{\lambda}$, and $\boldsymbol{\mu}$ as $\tilde{c}_{k \ell}=c_{k \ell}-\sum_{i \in N_{C}} a_{i k \ell}\left(\beta_{i k}+\right.$ $\left.\lambda_{i}\right)-\mu_{k}-\mu_{0}$. Note that $\tilde{c}_{k \ell} \geq 0, \ell \in \mathscr{R}_{k}, k \in N_{S}$, from the definition of values $\beta_{i k}$ (see inequalities (6.12)). From the definition of $\tilde{c}_{k \ell}$, we derive

$$
\sum_{k \in \bar{N}_{S}} \sum_{\ell \in J_{k}} \tilde{c}_{k \ell}=\sum_{k \in \bar{N}_{S}} \sum_{\ell \in J_{k}} c_{k \ell}-\sum_{k \in \bar{N}_{S}} \sum_{\ell \in J_{k}} \sum_{i \in N_{C}} a_{i k \ell}\left(\beta_{i k}+\lambda_{i}\right)-\sum_{k \in \bar{N}_{S}} \sum_{\ell \in J_{k}}\left(\mu_{k}+\mu_{0}\right) .
$$

Because any $F$ solution imposes that each customer is visited exactly once, we have

$$
\sum_{\ell \in J_{k}} \sum_{i \in N_{C}} a_{i k \ell}\left(\beta_{i k}+\lambda_{i}\right)=\sum_{i \in \bar{V}_{k}}\left(\beta_{i k}+\lambda_{i}\right), \quad k \in \bar{N}_{S} .
$$

Because $\left|J_{k}\right| \leq m_{k}, \mu_{k} \leq 0, k \in \bar{N}_{S}, \sum_{k \in L}\left|J_{k}\right| \leq m^{2}$, and $\mu_{0} \leq 0$, we have

$$
\sum_{k \in \bar{N}_{S}} \sum_{\ell \in J_{k}}\left(\mu_{k}+\mu_{0}\right)=\sum_{k \in \bar{N}_{S}}\left|J_{k}\right| \mu_{k}+\sum_{k \in \bar{N}_{S}}\left|J_{k}\right| \mu_{0} \geq \sum_{k \in \bar{N}_{S}} m_{k} \mu_{k}+m^{2} \mu_{0} .
$$

By adding and subtracting the term $\sum_{k \in L} g_{r}$ to the right-hand side of expression (6.19) and by using expressions (6.20) and (6.21), we obtain the following inequality

$$
\sum_{k \in \bar{N}_{S}} \sum_{\ell \in J_{k}} \tilde{c}_{k \ell} \leq \sum_{k \in L} g_{r}+\sum_{k \in \bar{N}_{S}} \sum_{\ell \in J_{k}} c_{k \ell}-\sum_{k \in L} g_{r}-\sum_{k \in \bar{N}_{S}} \sum_{i \in \bar{V}_{k}}\left(\beta_{i k}+\lambda_{i}\right)-\sum_{k \in \bar{N}_{S}} m_{k} \mu_{k}-m^{2} \mu_{0} .
$$

Note that $\sum_{k \in L} g_{r}+\sum_{k \in \bar{N}_{S}} \sum_{\ell \in J_{k}} c_{k \ell}=\bar{z}(F)$, so from expression (6.22) we derive

$$
\sum_{k \in \bar{N}_{S}} \sum_{\ell \in J_{k}} \tilde{c}_{k \ell} \leq \bar{z}(F)-\left(\sum_{k \in L} g_{r}+\sum_{k \in \bar{N}_{S}} \sum_{i \in \bar{V}_{k}}\left(\beta_{i k}+\lambda_{i}\right)+\sum_{k \in \bar{N}_{S}} m_{k} \mu_{k}+m^{2} \mu_{0}\right) .
$$

The second term of expression (6.23) is the cost, say $\tilde{z}(R F(\boldsymbol{\beta}, \boldsymbol{\lambda}, \boldsymbol{\mu}))$, of a feasible but nonnecessarily optimal $R F$ solution $(\tilde{\boldsymbol{\xi}}, \tilde{\boldsymbol{y}}, \tilde{\boldsymbol{q}})$ that is derived from the $F$ solution $(\overline{\boldsymbol{x}}, \overline{\boldsymbol{y}}, \overline{\boldsymbol{q}})$ by setting

1. $\tilde{\boldsymbol{y}}=\overline{\boldsymbol{y}}$;

2. $\tilde{\xi}_{i k}=1, i \in \bar{V}_{k}, k \in \bar{N}_{S}$;

3. $\tilde{\xi}_{i k}=0, i \in N_{C} \backslash \bar{V}_{k}, k \in \bar{N}_{S}$, and $\tilde{\xi}_{i k}=0, i \in N_{C}, k \in N_{S} \backslash \bar{N}_{S}$;

4. $\tilde{\boldsymbol{q}}=\overline{\boldsymbol{q}}$.

Because $\tilde{z}(R F(\boldsymbol{\beta}, \boldsymbol{\lambda}, \boldsymbol{\mu}))$ is greater than or equal to the optimal solution $\operatorname{cost} z(R F(\boldsymbol{\beta}, \boldsymbol{\lambda}, \boldsymbol{\mu}))$ of problem $R F$, from inequality (6.23) we derive

$$
\sum_{k \in \bar{N}_{S}} \sum_{\ell \in J_{k}} \tilde{c}_{k \ell} \leq \bar{z}(F)-z(R F(\boldsymbol{\beta}, \boldsymbol{\lambda}, \boldsymbol{\mu}))
$$


Because $\tilde{c}_{k \ell} \geq 0, \ell \in \mathscr{R}_{k}, k \in N_{S}$, from inequality (6.24) we have

$$
z(R F(\boldsymbol{\beta}, \boldsymbol{\lambda}, \boldsymbol{\mu})) \leq \bar{z}(F) \cdot \square
$$

From Theorem 6.1, the following corollary follows.

Corollary 1. Let $z_{U B}$ be a valid upper bound on the 2E-CVRP. For a given pair of vectors $\boldsymbol{\lambda}, \boldsymbol{\mu}$ and any solution $\boldsymbol{\beta}$ of inequalities (6.12), let $\tilde{c}_{k \ell}=c_{k \ell}-\sum_{i \in N_{C}} a_{i k \ell}\left(\beta_{i k}+\right.$ $\left.\lambda_{i}\right)-\mu_{k}-\mu_{0}$ be the reduced cost of $2^{\text {nd }}$-level route $\ell \in \mathscr{R}_{k}, k \in N_{S}$. Any optimal $2 \mathrm{E}$-CVRP solution of cost smaller than $z_{U B}$ cannot contain any $2^{\text {nd }}$-level route $\ell \in \mathscr{R}_{k}$, $k \in N_{S}$, of reduced cost $\tilde{c}_{k \ell} \geq z_{U B}-z(R F(\boldsymbol{\beta}, \boldsymbol{\lambda}, \boldsymbol{\mu}))$.

Proof. The corollary directly follows from inequality (6.24) because $\tilde{c}_{k \ell} \geq 0, \ell \in \mathscr{R}_{k}$, $k \in N_{S}$.

Theorem 6.2. The relation $\max _{\boldsymbol{\beta}, \boldsymbol{\lambda}, \boldsymbol{\mu}}\{z(R F(\boldsymbol{\beta}, \boldsymbol{\lambda}, \boldsymbol{\mu}))\} \geq z(L F)$ holds, and this inequality can be strict.

Proof. We show that, from any optimal $L F$ dual solution of cost $z(L F)$, two penalty vectors $\boldsymbol{\lambda}$ and $\boldsymbol{\mu}$ and a solution $\boldsymbol{\beta}$ of inequalities (6.12) such that $z(R F(\boldsymbol{\beta}, \boldsymbol{\lambda}, \boldsymbol{\mu})) \geq$ $z(L F)$ can be derived.

Let $\boldsymbol{u} \in \mathbb{R}^{\left|N_{C}\right|}, \boldsymbol{v} \in \mathbb{R}_{-}^{\left|N_{S}\right|}, v_{0} \in \mathbb{R}_{-}, \boldsymbol{\sigma} \in \mathbb{R}_{-}^{\left|N_{S}\right|}, \alpha_{0} \leq 0, \boldsymbol{\alpha} \in \mathbb{R}^{\left|N_{S}\right|}, \boldsymbol{\omega} \in \mathbb{R}_{-}^{|\mathscr{M}|}$, and $\boldsymbol{\vartheta} \in \mathbb{R}_{-}^{|\mathscr{M}|}$ be the dual variables associated with constraints (6.2)-(6.8) and with the continuous relaxation of constraints (6.10) (i.e., $0 \leq y_{r} \leq 1, r \in \mathscr{M}$ ). The dual of problem $L F$ is as follows.

$$
\begin{aligned}
& z(L F)=\max \sum_{i \in N_{C}} u_{i}+\sum_{k \in N_{S}} m_{k} v_{k}+m^{2} v_{0}+\sum_{k \in N_{S}} B_{k} \sigma_{k} \\
& +m^{1} \alpha_{0}+\sum_{r \in \mathscr{M}} \vartheta_{r} \\
& \text { s.t. } \sum_{i \in R_{k \ell}} u_{i}+v_{k}+v_{0}+w_{k \ell} \sigma_{k}-w_{k \ell} \alpha_{k} \leq c_{k \ell}, \quad \ell \in \mathscr{R}_{k}, k \in N_{S} \text {, } \\
& \alpha_{0}-Q_{1} \omega_{r}+\vartheta_{r} \leq g_{r}, \\
& r \in \mathscr{M}, \\
& \alpha_{k}+\omega_{r} \leq 0, \\
& u_{i} \in \mathbb{R} \text {, } \\
& k \in N_{S}, r \in \mathscr{M} \\
& v_{k} \leq 0, \alpha_{k} \in \mathbb{R}, \sigma_{k} \leq 0 \text {, } \\
& i \in N_{C} \text {, } \\
& v_{0} \leq 0, \alpha_{0} \leq 0 \text {, } \\
& k \in N_{S} \text {, } \\
& \omega_{r} \leq 0, \vartheta_{r} \leq 0 \text {, }
\end{aligned}
$$

Let $\boldsymbol{u}^{*}=\left(u_{1}^{*}, \ldots, u_{n_{c}}^{*}\right), \boldsymbol{v}^{*}=\left(v_{0}^{*}, v_{1}^{*}, \ldots, v_{n_{s}}^{*}\right), \boldsymbol{\sigma}^{*}=\left(\sigma_{1}^{*}, \ldots, \sigma_{n_{s}}^{*}\right), \boldsymbol{\alpha}^{*}=\left(\alpha_{0}^{*}, \alpha_{1}^{*}, \ldots, \alpha_{n_{s}}^{*}\right)$, $\boldsymbol{\omega}^{*}=\left(\omega_{1}^{*}, \ldots, \omega_{|\mathscr{M}|}^{*}\right)$, and $\boldsymbol{\vartheta}^{*}=\left(\vartheta_{1}^{*}, \ldots, \vartheta_{|\mathscr{M}|}^{*}\right)$ be an optimal dual solution of $L F$.

Define the vectors $\boldsymbol{\lambda}, \boldsymbol{\mu}$ and $\boldsymbol{\beta}$ with respect to $\boldsymbol{u}^{*}, \boldsymbol{v}^{*}, \boldsymbol{\sigma}^{*}$ and $\boldsymbol{\alpha}^{*}$ by setting $\lambda_{i}=0$, $i \in N_{C}, \mu_{k}=v_{k}^{*}, k \in N_{S}, \mu_{0}=v_{0}^{*}$, and $\beta_{i k}=u_{i}^{*}+q_{i}\left(\sigma_{k}^{*}-\alpha_{k}^{*}\right), i \in N_{C}, k \in N_{S}$. 
First, we show that vectors $\boldsymbol{\lambda}, \boldsymbol{\mu}$ and $\boldsymbol{\beta}$ as defined above provide a feasible solution of inequalities (6.12). Because $\sum_{i \in N_{C}} a_{i k \ell} q_{i}=w_{k \ell}$, we have

$$
\sum_{i \in N_{C}} a_{i k \ell} \beta_{i k}=\sum_{i \in N_{C}} a_{i k \ell} u_{i}^{*}+w_{k \ell} \sigma_{k}^{*}-w_{k \ell} \alpha_{k}^{*}, \quad \ell \in \mathscr{R}_{k}, k \in N_{S}
$$

Because of inequalities (6.26), from (6.33) we derive

$$
\sum_{i \in N_{C}} a_{i k \ell} \beta_{i k} \leq c_{k \ell}-v_{k}^{*}-v_{0}^{*}, \quad \ell \in \mathscr{R}_{k}, k \in N_{S}
$$

Inequalities (6.34) correspond to inequalities (6.12) because $\mu_{k}=v_{k}^{*}, k \in N_{S}, \mu_{0}=v_{0}^{*}$, and $\lambda_{i}=0, i \in N_{C}$.

Let $\left(\boldsymbol{\xi}^{*}, \boldsymbol{y}^{*}, \boldsymbol{q}^{*}\right)$ be an optimal $R F$ solution using $\boldsymbol{\lambda}, \boldsymbol{\mu}$ and $\boldsymbol{\beta}$ as defined above. We have

$$
\begin{gathered}
z(R F(\boldsymbol{\beta}, \boldsymbol{\lambda}, \boldsymbol{\mu}))=\sum_{k \in N_{S}} \sum_{i \in N_{C}}\left(u_{i}^{*}+q_{i}\left(\sigma_{k}^{*}-\alpha_{k}^{*}\right)\right) \xi_{i k}^{*}+\sum_{r \in \mathscr{M}} g_{r} y_{r}^{*}+\sum_{k \in N_{S}} m_{k} v_{k}^{*}+m^{2} v_{0}^{*} \\
=\sum_{i \in N_{C}} u_{i}^{*} \sum_{k \in N_{S}} \xi_{i k}^{*}+\sum_{k \in N_{S}} \sigma_{k}^{*} \sum_{i \in N_{C}} q_{i} \xi_{i k}^{*}-\sum_{k \in N_{S}} \alpha_{k}^{*} \sum_{i \in N_{C}} q_{i} \xi_{i k}^{*} \\
\quad+\sum_{r \in \mathscr{M}} g_{r} y_{r}^{*}+\sum_{k \in N_{S}} m_{k} v_{k}^{*}+m^{2} v_{0}^{*}
\end{gathered}
$$

From constraints (6.14), we have

$$
\sum_{i \in N_{C}} u_{i}^{*} \sum_{k \in N_{S}} \xi_{i k}^{*}=\sum_{i \in N_{C}} u_{i}^{*}
$$

From constraints (6.27), we derive

$$
\sum_{r \in \mathscr{M}} g_{r} y_{r}^{*} \geq-\sum_{r \in \mathscr{M}} Q_{1} \omega_{r}^{*} y_{r}^{*}+\alpha_{0}^{*} \sum_{r \in \mathscr{M}} y_{r}^{*}+\sum_{r \in \mathscr{M}} \vartheta_{r}^{*} y_{r}^{*}
$$

Because $\alpha_{0}^{*} \leq 0$, from inequality (6.6) we obtain

$$
\alpha_{0}^{*} \sum_{r \in \mathscr{M}} y_{r}^{*} \geq m^{1} \alpha_{0}^{*}
$$

and, because $\vartheta_{r}^{*} \leq 0$, we have

$$
\sum_{r \in \mathscr{M}} \vartheta_{r}^{*} y_{r}^{*} \geq \sum_{r \in \mathscr{M}} \vartheta_{r}^{*}
$$


Thus, from expressions (6.35), (6.36), (6.37), (6.38) and (6.39), we derive

$$
\begin{aligned}
z(R F(\boldsymbol{\beta}, \boldsymbol{\lambda}, \boldsymbol{\mu})) \geq \sum_{i \in N_{C}} u_{i}^{*} & +\sum_{k \in N_{S}} \sigma_{k}^{*} \sum_{i \in N_{C}} q_{i} \xi_{i k}^{*}-\sum_{k \in N_{S}} \alpha_{k}^{*} \sum_{i \in N_{C}} q_{i} \xi_{i k}^{*}-\sum_{r \in \mathscr{M}} Q_{1} \omega_{r}^{*} y_{r}^{*} \\
& +m^{1} \alpha_{0}^{*}+\sum_{r \in \mathscr{M}} \vartheta_{r}^{*}+\sum_{k \in N_{S}} m_{k} v_{k}^{*}+m^{2} v_{0}^{*} .
\end{aligned}
$$

From inequalities (6.8) and because $\omega_{r}^{*} \leq 0$, we obtain

$$
-\sum_{r \in \mathscr{M}} Q_{1} \omega_{r}^{*} y_{r}^{*} \geq-\sum_{r \in \mathscr{M}} \omega_{r}^{*} \sum_{k \in R_{r}} q_{k r}^{*}
$$

Because $\sum_{i \in N_{C}} q_{i} \xi_{i k}^{*}=\sum_{r \in \mathscr{M}} q_{k r}^{*}, k \in N_{S}$, from (6.41) we have

$$
\begin{array}{r}
\sum_{k \in N_{S}} \sigma_{k}^{*} \sum_{i \in N_{C}} q_{i} \xi_{i k}^{*}-\sum_{k \in N_{S}} \alpha_{k}^{*} \sum_{i \in N_{C}} q_{i} \xi_{i k}^{*}-\sum_{r \in \mathscr{M}} Q_{1} \omega_{r}^{*} y_{r}^{*} \geq \\
\sum_{k \in N_{S}}\left(\sigma_{k}^{*}-\alpha_{k}^{*}\right) \sum_{r \in \mathscr{M}} q_{k r}^{*}-\sum_{r \in \mathscr{M}} \omega_{r}^{*} \sum_{k \in R_{r}} q_{k r}^{*} .
\end{array}
$$

Notice that $\sum_{k \in N_{S}} \alpha_{k}^{*} \sum_{r \in \mathscr{M}} q_{k r}^{*}=\sum_{r \in \mathscr{M}} \sum_{k \in R_{r}} \alpha_{k}^{*} q_{k r}^{*}$. Because $\sigma_{k}^{*} \leq 0$ and $\sum_{r \in \mathscr{M}} q_{k r}^{*} \leq$ $B_{k}$ (see constraints (6.5)), we have $\sigma_{k}^{*} \sum_{r \in \mathscr{M}} q_{k r}^{*} \geq B_{k} \sigma_{k}^{*}, k \in N_{S}$. Thus, from (6.42), we derive

$$
\begin{array}{r}
\sum_{k \in N_{S}}\left(\sigma_{k}^{*}-\alpha_{k}^{*}\right) \sum_{r \in \mathscr{M}} q_{k r}^{*}-\sum_{r \in \mathscr{M}} \omega_{r}^{*} \sum_{k \in R_{r}} q_{k r}^{*} \geq \\
\sum_{k \in N_{S}} B_{k} \sigma_{k}^{*}-\sum_{r \in \mathscr{M}} \sum_{k \in R_{r}} \alpha_{k}^{*} q_{k r}^{*}-\sum_{r \in \mathscr{M}} \omega_{r}^{*} \sum_{k \in R_{r}} q_{k r}^{*} .
\end{array}
$$

Because of constraints (6.28), we have

$$
-\sum_{r \in \mathscr{M}} \sum_{k \in R_{r}} \alpha_{k}^{*} q_{k r}^{*}-\sum_{r \in \mathscr{M}} \omega_{r}^{*} \sum_{k \in R_{r}} q_{k r}^{*}=-\sum_{r \in \mathscr{M}} \sum_{k \in R_{r}}\left(\alpha_{k}^{*}+\omega_{r}^{*}\right) q_{k r}^{*} \geq 0 .
$$

From inequalities (6.40), (6.43) and (6.44) we obtain

$$
z(R F(\boldsymbol{\beta}, \boldsymbol{\lambda}, \boldsymbol{\mu})) \geq \sum_{i \in N_{C}} u_{i}^{*}+\sum_{k \in N_{S}} m_{k} v_{k}^{*}+m^{2} v_{0}^{*}+\sum_{k \in N_{S}} B_{k} \sigma_{k}^{*}+m^{1} \alpha_{0}^{*}+\sum_{r \in \mathscr{M}} \vartheta_{r}^{*}=z(L F) .
$$

Finally, notice that $z(R F(\boldsymbol{\beta}, \boldsymbol{\lambda}, \boldsymbol{\mu}))>z(L F)$ whenever the optimal dual solution $\left(\boldsymbol{u}^{*}, \boldsymbol{v}^{*}, \boldsymbol{\sigma}^{*}, \boldsymbol{\alpha}^{*}, \boldsymbol{\omega}^{*}, \boldsymbol{\vartheta}^{*}\right)$ does not saturate constraint (6.27) for some route $r \in \mathscr{M}$ that is in the optimal $R F$ solution (i.e., $y_{r}^{*}=1$ ) or/and constraint (6.28) for some satellite $k \in N_{S}$ and route $r \in \mathscr{M}$ such that $q_{k r}^{*}>0$. In these cases, inequality (6.37) or/and inequality (6.43) are strict and, consequently, $z(R F(\boldsymbol{\beta}, \boldsymbol{\lambda}, \boldsymbol{\mu}))>z(L F)$. 


\subsubsection{Relaxation $\overline{R F}$}

Solving $R F$ to optimality can be time-consuming, so we describe a further relaxation of $R F$, called $\overline{R F}$, that can be efficiently solved.

Let $\phi_{r w}$ be a lower bound on the cost for delivering a demand $w \in W_{r}$ to customers from the subsets of satellites $R_{r}$ visited by $1^{\text {st }}$-level route $r \in \mathscr{M}$. For a given vector $\boldsymbol{\beta}$ satisfying inequalities (6.12), the value $\phi_{r w}$ is defined as the optimal solution cost of the following continuous knapsack problem, called $K P(r, w)$

$$
\begin{aligned}
(K P(r, w)) \quad \phi_{r w}=\min & \sum_{i \in N_{C}} \min _{k \in R_{r}}\left\{\beta_{i k}\right\} z_{i} \\
\text { s.t. } & \sum_{i \in N_{C}} q_{i} z_{i}=w, \\
& 0 \leq z_{i} \leq 1, \quad i \in N_{C} .
\end{aligned}
$$

For each pair $(r, w)$, we denote by $z_{i}^{*}(r, w), i \in N_{C}$, the optimal solution of problem $K P(r, w)$, and we define $V(r, w)=\left\{i \in N_{C}: z_{i}^{*}(r, w)>0\right\}$. Let $\zeta_{r w}, r \in \mathscr{M}, w \in W_{r}$, be a binary variable equal to 1 if and only if $1^{\text {st }}$-level route $r$ delivering $w$ units of goods is in solution. Problem $\overline{R F}$ is defined as

$$
\begin{aligned}
& (\overline{R F}) \quad z(\overline{R F}(\boldsymbol{\beta}, \boldsymbol{\lambda}, \boldsymbol{\mu}))=\min \sum_{r \in \mathscr{M}} \sum_{w \in W_{r}}\left(g_{r}+\phi_{r w}\right) \zeta_{r w}+ \\
& \sum_{i \in N_{C}} \lambda_{i}+\sum_{k \in N_{S}} m_{k} \mu_{k}+m^{2} \mu_{0} \\
& \text { s.t. } \sum_{r \in \mathscr{M}} \sum_{w \in W_{r}} w \zeta_{r w}=q_{\text {tot }} \text {, } \\
& \sum_{w \in W_{r}} \zeta_{r w} \leq 1, \quad r \in \mathscr{M}, \\
& \zeta_{r w} \in\{0,1\}, \quad r \in \mathscr{M}, w \in W_{r} .
\end{aligned}
$$

Problem $\overline{R F}$ is an integer problem that can be conveniently solved by DP. The following theorem shows that $\overline{R F}$ is a relaxation of problem $R F$.

Theorem 6.3. The relation $z(\overline{R F}(\boldsymbol{\beta}, \boldsymbol{\lambda}, \boldsymbol{\mu})) \leq z(R F(\boldsymbol{\beta}, \boldsymbol{\lambda}, \boldsymbol{\mu}))$ holds for any solution $\boldsymbol{\beta}$ of inequalities (6.12) and for any penalty vectors $\boldsymbol{\lambda}$ and $\boldsymbol{\mu}$.

Proof. Let $(\overline{\boldsymbol{\xi}}, \overline{\boldsymbol{y}}, \overline{\boldsymbol{q}})$ be an $R F$ solution of $\operatorname{cost} \bar{z}(R F(\boldsymbol{\beta}, \boldsymbol{\lambda}, \boldsymbol{\mu}))$. This solution provides a feasible $\overline{R F}$ solution $\overline{\boldsymbol{\zeta}}$ of cost $\bar{z}(\overline{R F}(\boldsymbol{\beta}, \boldsymbol{\lambda}, \boldsymbol{\mu}))$ as follows. Let $\overline{\mathscr{M}}=\left\{r \in \mathscr{M}: \bar{y}_{r}=1\right\}$, and let $\bar{V}_{k}=\left\{i \in N_{C}: \bar{\xi}_{i k}=1\right\}, k \in N_{S}$. Define $\bar{\zeta}_{r w}=0, r \in \mathscr{M} \backslash \overline{\mathscr{M}}$ and $w \in W_{r}$. For any $r \in \mathscr{M}$, define $\bar{w}_{r}=\sum_{k \in R_{r}} \bar{q}_{r k}$. Set $\bar{\zeta}_{r \bar{w}_{r}}=1, r \in \overline{\mathscr{M}}$, and $\bar{\zeta}_{r w}=0, r \in \overline{\mathscr{M}}$, $w \neq \bar{w}_{r}$.

It is quite obvious to observe that $\bar{\zeta}$ satisfies constraints (6.47). To show that $\bar{\zeta}$ also satisfies constraints (6.46), we observe that, by adding up constraints 6.15 of problem 
$R F$, we obtain

$$
\sum_{k \in N_{S}} \sum_{r \in \mathscr{M}_{k}} \bar{q}_{r k}=\sum_{i \in N_{C}} q_{i} \sum_{k \in N_{S}} \bar{\xi}_{i k}
$$

Because $\bar{q}_{r k}=0, r \in \mathscr{M} \backslash \overline{\mathscr{M}}, k \in N_{S}$, and $r \in \overline{\mathscr{M}}, k \in N_{S} \backslash R_{r}$, we have

$$
\sum_{k \in N_{S}} \sum_{r \in \mathscr{M}_{k}} \bar{q}_{r k}=\sum_{r \in \overline{\mathscr{M}}} \sum_{k \in R_{r}} \bar{q}_{r k}
$$

Because of equations (6.14), from (6.49) and (6.50) we derive

$$
\sum_{r \in \bar{M}} \sum_{k \in R_{r}} \bar{q}_{r k}=\sum_{i \in N_{C}} q_{i}
$$

From the definition of $\bar{w}_{r}, r \in \mathscr{M}$, and of $\bar{\zeta}_{r w}, r \in \mathscr{M}, w \in W_{r}$, we have

$$
\sum_{r \in \overline{\mathscr{M}}} \sum_{k \in R_{r}} \bar{q}_{r k}=\sum_{r \in \overline{\mathscr{M}}} \sum_{w \in W_{r}} w \bar{\zeta}_{r w}
$$

which shows, together with equality (6.51), that $\bar{\zeta}$ also satisfies constraint (6.46).

For each $r \in \overline{\mathscr{M}}$, define $\theta_{i k r}, i \in N_{C}, k \in N_{S}$, by setting

$$
\theta_{i k r}= \begin{cases}0, & \text { if } \bar{\xi}_{i k}=0 \\ \frac{\bar{q}_{r k}}{\sum_{r \in \bar{M}_{k}} \bar{q}_{r k}}, & \text { if } \bar{\xi}_{i k}=1\end{cases}
$$

where $\overline{\mathscr{M}}_{k}=\mathscr{M}_{k} \cap \overline{\mathscr{M}}$.

In the following, we show that the values $\theta_{i k r}$, defined above, provide a feasible solution to problems $K P\left(r, \bar{w}_{r}\right), r \in \overline{\mathscr{M}}$. For each $r \in \overline{\mathscr{M}}$, define $\bar{z}_{i}\left(r, \bar{w}_{r}\right)=\sum_{k \in R_{r}} \theta_{i k r}, i \in N_{C}$. Since customer $i$ is assigned exactly to a satellite in any feasible $R F$ solution, from the definition of $\theta_{i k r}$, given by expression (6.52), we have $0 \leq \bar{z}_{i}\left(r, \bar{w}_{r}\right) \leq 1, i \in N_{C}$. Moreover, we have

$$
\sum_{i \in N_{C}} q_{i} \bar{z}_{i}\left(r, \bar{w}_{r}\right)=\sum_{i \in N_{C}} q_{i} \sum_{k \in R_{r}} \theta_{i k r}, \quad r \in \overline{\mathscr{M}}
$$

Because $\theta_{i k r}=0, i \in N_{C}, k \in N_{S} \backslash R_{r}$, we have

$$
\sum_{i \in N_{C}} q_{i} \sum_{k \in R_{r}} \theta_{i k r}=\sum_{k \in R_{r}}\left(\sum_{i \in \bar{V}_{k}} q_{i}\right) \theta_{i k r}=\sum_{k \in R_{r}}\left(\sum_{i \in \bar{V}_{k}} q_{i}\right) \frac{\bar{q}_{r k}}{\sum_{r \in \overline{\mathscr{M}}_{k}} \bar{q}_{r k}}, \quad r \in \overline{\mathscr{M}} .
$$

Since $\sum_{r \in \overline{\mathscr{M}}_{k}} \bar{q}_{r k}=\sum_{i \in \bar{V}_{k}} q_{i}$, from (6.53), (6.54) and the definition of $\bar{w}_{r}$, we obtain

$$
\sum_{i \in N_{C}} q_{i} \bar{z}_{i}\left(r, \bar{w}_{r}\right)=\sum_{k \in R_{r}} \bar{q}_{r k}=\bar{w}_{r}, \quad r \in \overline{\mathscr{M}} .
$$


The cost $\bar{\phi}_{r \bar{w}_{r}}$ of solution of problem $K P\left(r, \bar{w}_{r}\right)$ is

$$
\bar{\phi}_{r \bar{w}_{r}}=\sum_{i \in N_{C}} \min _{k \in R_{r}}\left\{\beta_{i k}\right\} \bar{z}_{i}\left(r, \bar{w}_{r}\right), \quad r \in \overline{\mathscr{M}} .
$$

Thus, the cost $\bar{z}(\overline{R F}(\boldsymbol{\beta}, \boldsymbol{\lambda}, \boldsymbol{\mu}))$ of the $\overline{R F}$ solution $\overline{\boldsymbol{\zeta}}$ obtained from the $R F$ solution $(\overline{\boldsymbol{\xi}}, \overline{\boldsymbol{y}}, \overline{\boldsymbol{q}})$ is

$$
\bar{z}(\overline{R F}(\boldsymbol{\beta}, \boldsymbol{\lambda}, \boldsymbol{\mu}))=\sum_{r \in \bar{M}} \bar{\phi}_{r \bar{w}_{r}}+\sum_{r \in \overline{\mathscr{M}}} g_{r}+\sum_{i \in N_{C}} \lambda_{i}+\sum_{k \in N_{S}} m_{k} \mu_{k}+m^{2} \mu_{0} .
$$

The cost $\bar{z}(R F(\boldsymbol{\beta}, \boldsymbol{\lambda}, \boldsymbol{\mu}))$ of the $R F$ solution $(\overline{\boldsymbol{\xi}}, \overline{\boldsymbol{y}}, \overline{\boldsymbol{q}})$ is

$$
\bar{z}(R F(\boldsymbol{\beta}, \boldsymbol{\lambda}, \boldsymbol{\mu}))=\sum_{i \in N_{C}} \sum_{k \in N_{S}} \beta_{i k} \bar{\xi}_{i k}+\sum_{r \in \overline{\mathscr{M}}} g_{r}+\sum_{i \in N_{C}} \lambda_{i}+\sum_{k \in N_{S}} m_{k} \mu_{k}+m^{2} \mu_{0} .
$$

From the definition of $\theta_{i k r}$ (see expression (6.52)), we have

$$
\bar{\xi}_{i k}=\sum_{r \in \overline{\mathscr{M}}_{k}} \theta_{i k r}
$$

From (6.55) and (6.56) we obtain

$$
\bar{z}(R F(\boldsymbol{\beta}, \boldsymbol{\lambda}, \boldsymbol{\mu}))=\sum_{r \in \overline{\mathscr{M}}}\left(\sum_{i \in N_{C}} \sum_{k \in R_{r}} \beta_{i k} \theta_{i k r}+g_{r}\right)+\sum_{i \in N_{C}} \lambda_{i}+\sum_{k \in N_{S}} m_{k} \mu_{k}+m^{2} \mu_{0} .
$$

It is quite clear that the following inequalities hold

$$
\sum_{i \in N_{C}} \sum_{k \in R_{r}} \beta_{i k} \theta_{i k r} \geq \sum_{i \in N_{C}} \min _{k \in R_{r}}\left\{\beta_{i k}\right\} \sum_{k \in R_{r}} \theta_{i k r}, \quad r \in \overline{\mathscr{M}}
$$

Thus, using the definition of $\bar{z}_{i}\left(r, \bar{w}_{r}\right)$, inequalities (6.58) become

$$
\sum_{i \in N_{C}} \sum_{k \in R_{r}} \beta_{i k} \theta_{i k r} \geq \sum_{i \in N_{C}} \min _{k \in R_{r}}\left\{\beta_{i k}\right\} \bar{z}_{i}\left(r, \bar{w}_{r}\right)=\bar{\phi}_{r} \bar{w}_{r}, \quad r \in \overline{\mathscr{M}}
$$

Finally, from expression (6.57) and inequalities (6.59), we obtain

$$
\bar{z}(R F(\boldsymbol{\beta}, \boldsymbol{\lambda}, \boldsymbol{\mu})) \geq \sum_{r \in \overline{\mathscr{M}}}\left(\bar{\phi}_{r} \bar{w}_{r}+g_{r}\right)+\sum_{i \in N_{C}} \lambda_{i}+\sum_{k \in N_{S}} m_{k} \mu_{k}+m^{2} \mu_{0}=\bar{z}(\overline{R F}(\boldsymbol{\beta}, \boldsymbol{\lambda}, \boldsymbol{\mu})) \cdot \square
$$

Because of Theorem 6.3 and Corollary 1, any optimal 2E-CVRP solution of cost smaller than a known upper bound $z_{U B}$ cannot contain any $2^{\text {nd }}$-level route $\ell \in \mathscr{R}_{k}, k \in N_{S}$, of reduced cost $\tilde{c}_{k \ell} \geq z_{U B}-z(\overline{R F}(\boldsymbol{\beta}, \boldsymbol{\lambda}, \boldsymbol{\mu}))$, where $\tilde{c}_{k \ell}$ is defined as above. 
A valid lower bound $L D 1$ on the $2 \mathrm{E}-\mathrm{CVRP}$ can be computed as the cost of a nearoptimal solution of problem

$$
L D 1=\max _{\boldsymbol{\beta}, \boldsymbol{\lambda}, \boldsymbol{\mu}}\{z(\overline{R F}(\boldsymbol{\beta}, \boldsymbol{\lambda}, \boldsymbol{\mu}))\} .
$$

\subsection{Lower bound $L D 1$ and bounding procedure $D P^{1}$}

In this section, we describe a bounding procedure, called $D P^{1}$, and a heuristic algorithm based on relaxation $\overline{R F}$ to compute lower and upper bounds $L D 1$ and $U B 1$, respectively. Procedure $D P^{1}$ finds a near-optimal solution of problem (6.60) and uses a DP algorithm to solve problem $\overline{R F}$.

Bounding procedure $D P^{1}$ is based on a relaxation of the $2 \mathrm{E}$-CVRP, where the $2^{\text {nd }}$-level route sets $\mathscr{R}_{k}, k \in N_{S}$, are enlarged to also contain nonnecessarily elementary routes. The method used by $D P^{1}$ to find a feasible solution of inequalities (6.12) is based on the following theorem.

Theorem 6.4. Let us associate penalties $\lambda_{i} \in \mathbb{R}, i \in N_{C}$, with constraints (6.2), $\mu_{k} \in \mathbb{R}_{-}, k \in N_{S}$, with constraints (6.3), and $\mu_{0} \in \mathbb{R}_{-}$with constraint (6.4). Let $\widehat{\mathscr{R}}_{k} \supseteq \mathscr{R}_{k}$ be the index set of nonnecessarily elementary routes for satellite $k$. A feasible solution $\beta_{i k}$ of inequalities (6.12) is given by

$$
\beta_{i k}=q_{i} \min _{\ell \in \widehat{\mathscr{R}}_{i k}}\left\{\frac{c_{k \ell}-\sum_{i \in N_{C}} a_{i k \ell} \lambda_{i}-\mu_{k}-\mu_{0}}{\sum_{i \in N_{C}} a_{i k \ell} q_{i}}\right\}, \quad i \in N_{C}, k \in N_{S} .
$$

Proof. Consider route $\ell \in \widehat{\mathscr{R}}_{k}$ of a given satellite $k \in N_{S}$. From expressions (6.61), we derive

$$
\beta_{i k} \leq \frac{q_{i}\left(c_{k \ell}-\sum_{i \in N_{C}} a_{i k \ell} \lambda_{i}-\mu_{k}-\mu_{0}\right)}{\sum_{i \in N_{C}} a_{i k \ell} q_{i}}, \quad i \in R_{k \ell} .
$$

By summing up inequalities (6.62) for all $i \in R_{k \ell}$, we obtain

$$
\begin{array}{r}
\sum_{i \in R_{k \ell}} a_{i k \ell} \beta_{i k} \leq \sum_{i \in R_{k \ell}} \frac{a_{i k \ell} q_{i}\left(c_{k \ell}-\sum_{i \in N_{C}} a_{i k \ell} \lambda_{i}-\mu_{k}-\mu_{0}\right)}{\sum_{i \in N_{C}} a_{i k \ell} q_{i}} \\
=c_{k \ell}-\sum_{i \in N_{C}} a_{i k \ell} \lambda_{i}-\mu_{k}-\mu_{0}, \quad \ell \in \widehat{\mathscr{R}}_{k}, k \in N_{S} \cdot \square
\end{array}
$$

In procedure $D P^{1}$, the route set $\widehat{\mathscr{R}}_{k}$ is defined as the set of $n g$-routes introduced in $\S 4.3 .3$ that are shortly described below.

Let $N_{i} \subseteq N_{C}, i \in N_{C}$, be a set of selected customers for customer $i$ (according to some criterion), such that $N_{i} \ni i$ and $\left|N_{i}\right| \leq \Delta\left(N_{i}\right)$, where $\Delta\left(N_{i}\right)$ is a parameter. The sets $N_{i}$ allow us to associate with each path $P=\left(k, i_{1}, \ldots, i_{t}\right)$ that starts from 
satellite $k \in N_{S}$, visits vertices $i_{1}, \ldots, i_{t} \in N_{C}$, and ends at vertex $i_{t}$, the subset $\Pi(P)$ containing $i_{t}$ and every customer $i_{s}, s=1, \ldots, t-1$, of $P$ that belongs to all sets $N_{s+1}, \ldots, N_{i_{t}}$ associated with the customers $i_{s+1}, \ldots, i_{t}$ visited after $i_{s}$. The set $\Pi(P)$ is defined as

$$
\Pi(P)=\left\{i_{s}: i_{s} \in \bigcap_{j=s+1}^{t} N_{i_{j}}, s=1, \ldots, t-1\right\} \bigcup\left\{i_{t}\right\} .
$$

A forward $n g$-path $(N G, k, q, i)$ is a nonnecessarily elementary path $P=\left(k, i_{1}, \ldots\right.$, $\left.i_{t-1}, i_{t}=i\right)$ that starts from satellite $k \in N_{S}$, ends at customer $i$, visits a subset of customers of total demand equal to $q$, and such that $N G=\Pi(P)$ and $i \notin \Pi\left(P^{\prime}\right)$, where $P^{\prime}=\left(k, i_{1}, \ldots, i_{t-1}\right)$. An $(N G, k, q, i)$-route (or simply $n g$-route) is obtained by adding edge $\{i, k\}$ to an $n g$-path $(N G, k, q, i)$.

Algorithm $D P^{1}$ uses column generation to solve equations (6.61) and subgradient optimization to solve problem (6.60).

\subsubsection{Description of Procedure $D P^{1}$}

To solve equations (6.61), procedure $D P^{1}$ uses a limited set $\overline{\mathscr{R}}_{k} \subseteq \widehat{\mathscr{R}}_{k}, k \in N_{S}$, of $n g$ routes. Procedure $D P^{1}$ initializes each set $\overline{\mathscr{R}}_{k}$ with all single-customer routes $(k, i, k)$, $i \in N_{C}$, and sets $\boldsymbol{\lambda}=\mathbf{0}, \boldsymbol{\mu}=\mathbf{0}, L D 1=0$ and $U B 1=\infty$. Procedure $D P^{1}$ executes an a-priori defined number (Maxit1) of macro iterations where, at each macro iteration, the following steps are performed.

(1) Initialize $z^{*}=0$, and perform Maxit2 iterations of the following steps.

(i) Compute values $\beta_{i k}, i \in N_{C}, k \in N_{S}$, through expression (6.61), where each set $\widehat{\mathscr{R}}_{k}$ is replaced with set $\overline{\mathscr{R}}_{k}, k \in N_{S}$.

(ii) Solve $\overline{R F}$ using values $\beta_{i k}$ as described in $\S 6.5 .2$. If $z(\overline{R F}(\boldsymbol{\beta}, \boldsymbol{\lambda}, \boldsymbol{\mu}))>z^{*}$, then update $z^{*}=z(\overline{R F}(\boldsymbol{\beta}, \boldsymbol{\lambda}, \boldsymbol{\mu})), \boldsymbol{\beta}^{*}=\boldsymbol{\beta}, \boldsymbol{\lambda}^{*}=\boldsymbol{\lambda}$ and $\boldsymbol{\mu}^{*}=\boldsymbol{\mu}$.

(iii) Update penalty vector $\boldsymbol{\lambda}$ and $\boldsymbol{\mu}$ as described in $\S 6.5 .3$.

(2) Generate a set of $n g$-routes $\mathscr{N}_{k} \subseteq \widehat{\mathscr{R}}_{k} \backslash \overline{\mathscr{R}}_{k}, k \in N_{S}$, for which inequalities (6.12) are violated by $\boldsymbol{\beta}^{*}, \boldsymbol{\lambda}^{*}$ and $\boldsymbol{\mu}^{*}$ as described in $\S 6.5 .4$. There are two cases

(i) If $\mathscr{N}_{k}=\varnothing$, for each satellite $k \in N_{S}$. If $L D 1<z^{*}$, then update $L D 1=z^{*}$, $\boldsymbol{\beta}^{\mathbf{1}}=\boldsymbol{\beta}^{*}, \boldsymbol{\lambda}^{\mathbf{1}}=\boldsymbol{\lambda}^{*}, \boldsymbol{\mu}^{\mathbf{1}}=\boldsymbol{\mu}^{*}$, and execute the heuristic algorithm described in $\S 6.5 .5$ producing upper bound $z_{U B}$. Update $U B 1=\min \left\{U B 1, z_{U B}\right\}$.

(ii) If $\mathscr{N}_{k} \neq \varnothing$, for some satellite $k \in N_{S}$, then update $\overline{\mathscr{R}}_{k}=\overline{\mathscr{R}}_{k} \cup \mathscr{N}_{k}$.

Notice that $\boldsymbol{\beta}^{\mathbf{1}}, \boldsymbol{\lambda}^{\mathbf{1}}$ and $\boldsymbol{\mu}^{\mathbf{1}}$ are the vectors producing lower bound $L D 1$ in problem (6.60). 


\subsubsection{Solving Problem $\overline{R F}$}

Problem $\overline{R F}$ can be solved by DP as follows. Let $h(r, w)$ be the optimal solution cost of $\overline{R F}$ obtained by using the $1^{s t}$-level routes $1, \ldots, r, 0 \leq r \leq|\mathscr{M}|$, and replacing $q_{\text {tot }}$ in equation (6.46) with $w \in \mathbb{Z}_{+}, w^{\text {min }} \leq w \leq q_{\text {tot }}$. The DP recursion for computing functions $h(r, w), r=1, \ldots,|\mathscr{M}|, w^{\text {min }} \leq w \leq q_{t o t}$, is

$$
h(r, w)=\min \left\{h(r-1, w), \min _{w^{\min } \leq w^{\prime} \leq \min \left\{w, w_{r}^{\max }\right\}}\left\{h\left(r-1, w-w^{\prime}\right)+g_{r}+\phi_{r w^{\prime}}\right\}\right\} .
$$

The recursion is initialized by setting $h(r, 0)=0, r=0, \ldots,|\mathscr{M}|$, and $h(0, w)=\infty$, $w=1, \ldots, q_{\text {tot }}$. The $\overline{R F}$ optimal solution cost is $z(\overline{R F}(\boldsymbol{\beta}, \boldsymbol{\lambda}, \boldsymbol{\mu}))=h\left(|\mathscr{M}|, q_{\text {tot }}\right)$.

Let $\bar{z}$ be an upper bound on $z(\overline{R F}(\boldsymbol{\beta}, \boldsymbol{\lambda}, \boldsymbol{\mu}))$. The number of states $(r, w)$ to generate in order to compute $z(\overline{R F}(\boldsymbol{\beta}, \boldsymbol{\lambda}, \boldsymbol{\mu}))$ can be reduced by using bounding functions $l b(r, w)$, described below, to eliminate any state $(r, w)$ that cannot lead to any $\overline{R F}$ solution of cost smaller than $\bar{z}$.

We denote by $l b(r, w)$ a lower bound on the optimal solution cost of problem $\overline{R F}$ where the set $\mathscr{M}$ is replaced with the subset $\{r, r+1, \ldots,|\mathscr{M}|\}$ and $q_{\text {tot }}$ with $w$. Let $\alpha_{r}=\min _{w^{\min } \leq w \leq w_{r}^{\max }}\left\{\left(g_{r}+\phi_{r w}\right) / w\right\}, r \in \mathscr{M}$. By assuming that the routes in the set $\mathscr{M}$ are indexed so that $\alpha_{1} \leq \alpha_{2} \leq \ldots \leq \alpha_{|\mathscr{M}|}$, functions $l b(r, w)$ can be computed using the following backward recursion.

Initialize (i) $l b(r, 0)=0, r=1, \ldots,|\mathscr{M}|$, (ii) $l b(r, w)=\infty, 0<w<w^{\text {min }}, r=$ $1, \ldots,|\mathscr{M}|$, (iii) $l b(|\mathscr{M}|, w)=w \alpha_{|\mathscr{M}|}, w \in W_{|\mathscr{M}|}$, and (iv) $l b(|\mathscr{M}|, w)=\infty, w_{r}^{\max }<$ $w \leq q_{\text {tot }}$.

For each $1^{\text {st }}$-level route $r=|\mathscr{M}|-1,|\mathscr{M}|-2, \ldots, 1$ and each load $w^{\text {min }} \leq w \leq q_{\text {tot }}$ compute

$$
l b(r, w)= \begin{cases}w \alpha_{r} & \text { if } w \leq w_{r}^{\max } \\ w_{r}^{\text {max }} \alpha_{r}+l b\left(r+1, w-w_{r}^{\text {max }}\right) & \text { if } w_{r}^{\text {max }}+1 \leq w \leq q_{\text {tot }} .\end{cases}
$$

Thus, a state $(r, w), r<|\mathscr{M}|$, is fathomed if $h(r, w)+l b\left(r+1, q_{t o t}-w\right) \geq \bar{z}$.

In performing recursion (6.63), the upper bound $\bar{z}$ is initialized as $\bar{z}=U B 1$ and dynamically updated, at the end of stage $r$, as $\bar{z}=\min \left\{\bar{z}, h\left(r, q_{t o t}\right)\right\}$.

\subsubsection{Computing a subgradient}

Usual backtracking can be used to derive the $\overline{R F}$ solution $\boldsymbol{\zeta}$ of cost $h\left(|\mathscr{M}|, q_{t o t}\right)$. Given $\boldsymbol{\zeta}$ and the sets $V(r, w)$, as defined in $\S 6.4 .2$, associated to $\phi_{r w}$, we derive the index sets $\widetilde{\mathscr{R}}_{k} \subseteq \overline{\mathscr{R}}_{k}, k \in N_{S}$, of the $2^{n d}$-level routes in solution and the index $\ell(i, k)$ of the route in $\widetilde{\mathscr{R}}_{k}$ associated with $\beta_{i k}$ as follows. 
(i) Initialize $\widetilde{\mathscr{R}}_{k}=\varnothing, k \in N_{S}$, and $\ell(i, k)=0, i \in N_{C}, k \in N_{S}$;

(ii) Repeat the following steps for each $1^{\text {st }}$-level route $r \in \mathscr{M}$ such that $\zeta_{r w}=1$ for some load $w \in W_{r}$

(a) Compute $\bar{k}(i)=\operatorname{argmin}_{k \in R_{r}}\left\{\beta_{i k}\right\}, i \in V(r, w)$. Let $\ell(i, \bar{k}(i)), i \in V(r, w)$, be the index of the route in $\widehat{\mathscr{R}}_{k}$ associated with $\beta_{i} \bar{k}(i)$ in expressions (6.61);

(b) For each $i \in V(r, w)$, set $\widetilde{\mathscr{R}}_{\bar{k}(i)}=\widetilde{\mathscr{R}}_{\bar{k}(i)} \cup\{\ell(i, \bar{k}(i))\}$.

A subgradient to function $z(\overline{R F}(\boldsymbol{\beta}, \boldsymbol{\lambda}, \boldsymbol{\mu}))$, at point $(\boldsymbol{\lambda}, \boldsymbol{\mu})$, can be computed as follows. Let $\tilde{\boldsymbol{x}}$ be a vector whose components are computed as

$$
\tilde{x}_{k \ell}=\sum_{i \in N_{C}: \ell(i, k)=\ell} \frac{a_{i k \ell} q_{i}}{\sum_{i \in N_{C}} a_{i k \ell} q_{i}}, \quad \ell \in \widetilde{\mathscr{R}}_{k}, k \in N_{S},
$$

and let

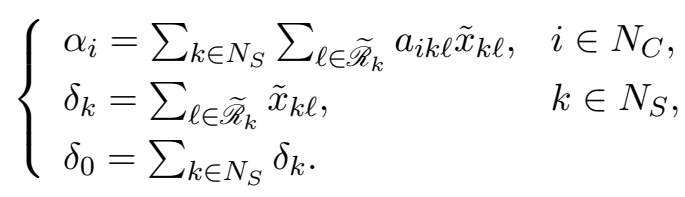

Then, penalty vectors $\boldsymbol{\lambda}$ and $\boldsymbol{\mu}$ are modified as

$$
\begin{cases}\lambda_{i}=\lambda_{i}-\epsilon \gamma\left(\alpha_{i}-1\right), & i \in N_{C}, \\ \mu_{k}=\min \left\{0, \mu_{k}-\epsilon \gamma\left(\delta_{k}-m_{k}\right)\right\}, & k \in N_{S}, \\ \mu_{0}=\min \left\{0, \mu_{0}-\epsilon \gamma\left(\delta_{0}-m^{2}\right)\right\}, & \end{cases}
$$

where $\epsilon$ is a positive constant and $\gamma$ is defined as

$$
\gamma=\frac{0.2 z(\overline{R F}(\boldsymbol{\beta}, \boldsymbol{\lambda}, \boldsymbol{\mu}))}{\sum_{i \in N_{C}}\left(\alpha_{i}-1\right)^{2}+\sum_{k \in N_{S}}\left(\delta_{k}-m_{k}\right)^{2}+\left(\delta_{0}-m^{2}\right)^{2}} .
$$

\subsubsection{Generating the $n g$-route set $\mathscr{N}_{k}$ for a given satellite $k$}

We describe the procedure to generate, for a given satellite $k \in N_{S}$, the set of $n g$-routes $\mathscr{N}_{k} \subseteq \widehat{\mathscr{R}}_{k}$ that violate inequalities (6.12) for given vectors $\boldsymbol{\beta}^{*}, \boldsymbol{\lambda}^{*}$ and $\boldsymbol{\mu}^{*}$, when the route set $\mathscr{R}_{k}$ is replaced by the route set $\widehat{\mathscr{R}}_{k}$.

Define the modified edge costs $\bar{d}_{i j}=d_{i j}-\frac{1}{2}\left(\beta_{i k}^{*}+\lambda_{i}^{*}+H_{k} q_{i}\right)-\frac{1}{2}\left(\beta_{j k}^{*}+\lambda_{j}^{*}+H_{k} q_{j}\right)$ and the sets $N_{i} \subseteq N_{C}, i \in N_{C}$, to contain the $\Delta\left(N_{i}\right)$ nearest customers to $i$ according to $d_{i j}$.

Let $f(N G, k, q, i)$ be the cost of a least-cost $n g$-path $(N G, k, q, i)$ using the modified edge cost $\bar{d}_{i j}$. Functions $f(N G, k, q, i)$ are computed using the DP recursions described in $\S 4.3 .3$ on the state-space graph $\mathscr{H}=(\mathscr{V}, \Psi)$, defined for a given satellite $k \in N_{S}$, 
as

$\mathscr{V}=\left\{(N G, k, q, i): q_{i} \leq q \leq Q_{2}, \forall N G \subseteq N_{i}\right.$ s.t. $N G \ni i$ and $\left.\sum_{j \in N G} q_{j} \leq q, \forall i \in N_{C}\right\}$

$\Psi=\left\{\left(\left(N G^{\prime}, k, q^{\prime}, j\right),(N G, k, q, i)\right): \forall\left(N G^{\prime}, k, q^{\prime}, j\right) \in \Psi^{-1}(N G, k, q, i), \forall(N G, k, q, i) \in \mathscr{E}\right\}$,

where $\Psi^{-1}(N G, k, q, i)=\left\{\left(N G^{\prime}, k, q-q_{i}, j\right): \forall N G^{\prime} \subseteq N_{j}\right.$ s.t. $N G^{\prime} \ni j$ and $N G^{\prime} \cap$ $N_{i}=N G \backslash\{i\}, j \in N_{C}$ s.t. $\{i, j\} \in E$ if $i<j$ or $\{j, i\} \in E$ if $\left.j<i\right\}$.

Let $r(i, k)=\min _{(N G, k, q, i) \in \mathscr{V}}\left\{f(N G, k, q, i)-\mu_{k}^{*}-\mu_{0}^{*}+\bar{d}_{i k}\right\}$ be the cost of a least-cost $n g$ route visiting customer $i \in N_{C}$ immediately before arriving at satellite $k$. The route set $\mathscr{N}_{k}$ contains the $n g$-routes corresponding to $r(i, k)<0$, for each customer $i \in N_{C}$.

\subsubsection{A Lagrangean heuristic}

Procedure $D P^{1}$ is interwoven with a heuristic algorithm that produces a feasible $2 \mathrm{E}$ CVRP solution of cost $z_{U B}$ using the $2^{n d}$-level route sets $\widetilde{\mathscr{R}}_{k}, k \in N_{S}$, and vector $\tilde{\boldsymbol{x}}$ associated with an $\overline{R F}$ solution (see $\S 6.5 .3$ ). First, the routes in $\widetilde{\mathscr{R}}_{k}, k \in N_{S}$, are modified with the objective of obtaining a solution vector $\boldsymbol{x}$ satisfying constraints (6.2)(6.5). Then, the solution vector $\boldsymbol{x}$ is used to derive solution vectors $\boldsymbol{y}$ and $\boldsymbol{q}$ such that $(\boldsymbol{x}, \boldsymbol{y}, \boldsymbol{q})$ represents a feasible $2 \mathrm{E}-\mathrm{CVRP}$ solution.

A step-by-step description of the heuristic algorithm is the following.

1) Initialization. Let $\widetilde{\mathscr{R}}=\cup_{k \in N_{S}} \widetilde{\mathscr{R}}_{k}$. Initialize $S O L=\varnothing$ and $\delta(i)=0, i \in N_{C}$.

2) Extract a subset of routes $S O L \subseteq \widetilde{\mathscr{R}}$. Let $\ell^{*}$ be the route of $\widetilde{\mathscr{R}}$ where $\tilde{x}_{\pi_{\ell^{*}} \ell^{*}}=$ $\max \left\{\tilde{x}_{\pi_{\ell} \ell}: \ell \in \widetilde{\mathscr{R}}\right\}$. Remove $\ell^{*}$ from $\widetilde{\mathscr{R}}$. If $\delta(i)=0$, for some $i \in R_{\pi_{\ell^{*} \ell^{*}}}$, then update $S O L=S O L \cup\left\{\ell^{*}\right\}$ and $\delta(i)=\delta(i)+1, i \in R_{\pi_{\ell^{*} \ell^{*}}}$. Repeat Step 1 until $\widetilde{\mathscr{R}}=\varnothing$.

3) Modify the route set $S O L$. Remove from $S O L$ any route $\ell \in S O L$ such that $\delta(i)>1, i \in R_{\pi_{\ell} \ell}$, and update $\delta(i)=\delta(i)-1$. For each $\ell \in S O L$, compute the savings that can be achieved by removing from route $\ell$ every customer $i \in R_{\pi_{\ell} \ell}$ having $\delta(i)>1$. Let $\ell^{*} \in S O L$ be the route of maximum saving. Remove from route $\ell^{*}$ every customer $i \in R_{\pi_{\ell^{*}} \ell^{*}}$ with $\delta(i)>1$, and update $\delta(i)=\delta(i)-1$. Repeat Step 3 until $\delta(i) \leq 1$, for each $i \in N_{C}$.

4) Insert unrouted customers. For each unrouted customer $i$ (i.e., $\delta(i)=0$ ) perform the following operations. Compute the minimum extra-mileage exm $(i, \ell)$ for inserting $i$ in route $\ell \in S O L$. We set $\operatorname{exm}(i, \ell)=\infty$ if the total load of the resulting route $\ell$ exceeds the vehicle capacity $Q_{2}$. Let $\ell^{*}$ be such that $\operatorname{exm}\left(i, \ell^{*}\right)=\min _{\ell \in S O L}[\operatorname{exm}(i, \ell)]$. If $\operatorname{exm}\left(i, \ell^{*}\right)=\infty$, then set $z_{U B}=\infty$ and stop; otherwise, insert customer $i$ in route $\ell^{*}$ in the position of cost $\operatorname{exm}\left(i, \ell^{*}\right)$, and set $\delta(i)=1$. 
5) Define the $F$ solution $\boldsymbol{x}$. Define $x_{\pi_{\ell} \ell}=1$, for each route $\ell \in S O L$, and $x_{\pi_{\ell} \ell}=0$, for each route $\ell \in \mathscr{R} \backslash S O L$. If $\boldsymbol{x}$ does not satisfy constraints (6.3)-(6.5), then set $z_{U B}=\infty$ and the algorithm terminates.

6) Improve the cost of the routes in SOL. The post-optimization procedure for improving the total cost of the routes in $S O L$ applies the following procedure in the order specified below.

(a) Exchange of one customer between two routes of $S O L$. For each customer $i \in$ $N_{C}$, compute the saving move $(i, \ell)$ achieved by removing $i$ from its current route $\ell_{i}$ and inserting $i$ in the least-cost position of route $\ell \in S O L$. Set move $\left(i, \ell_{i}\right)=0$ and $\operatorname{move}(i, \ell)=-\infty$ if the load of the resulting route $\ell$ violates constraint (6.5) for satellite $\pi_{\ell}$ or if customer $i$ cannot be inserted in route $\ell$ without violating the vehicle capacity $Q_{2}$. Let $i^{*}$ and $\ell^{*}$ be determined such that move $\left(i^{*}, \ell^{*}\right)=$ $\max \left[\operatorname{move}(i, \ell): i \in N_{C}, \ell \in S O L\right]$. If move $\left(i^{*}, \ell^{*}\right)>0$, then remove customer $i^{*}$ from its current route and insert it in the best position of route $\ell^{*}$. This procedure is repeated until move $\left(i^{*}, \ell^{*}\right) \leq 0$.

(b) Exchange of two customers between two routes of $S O L$. For all pairs of routes $\ell, \ell^{\prime} \in S O L$ and for each pair of customers $i \in R_{\pi_{\ell} \ell}$ and $j \in R_{\pi_{\ell^{\prime} \ell^{\prime}}}$, compute the saving $\operatorname{sav}(i, j)$ obtained by moving customer $i$ from route $\ell$ to route $\ell^{\prime}$ and customer $j$ from route $\ell^{\prime}$ to route $\ell$. We set $\operatorname{sav}(i, j)=-\infty$ if the exchange violates constraint (6.5) for one of the two satellites $\pi_{\ell}, \pi_{\ell^{\prime}}$ or if the total load of one of the two routes exceeds the vehicle capacity $Q_{2}$. The two customers, $i^{*}$ and $j^{*}$, producing the maximum saving are then exchanged if $\operatorname{sav}\left(i^{*}, j^{*}\right)>0$. This procedure is repeated until $\operatorname{sav}\left(i^{*}, j^{*}\right) \leq 0$. Whenever this procedure improves the solution, then the post-optimization routing is restarted from the beginning.

(c) Optimize each route $\ell \in S O L$ using a 3-optimal method.

7) Constructing a feasible $2 E-C V R P$ solution. Let $\omega_{k}=\sum_{\ell \in \mathscr{R}_{k}} w_{k \ell} x_{k \ell}, k \in N_{S}$, be the total demand associated with satellite $k$ by the solution vector $\boldsymbol{x}$ defined above. We solve the following integer problem to optimality

$$
\begin{array}{rlr}
(F(\boldsymbol{x})) z(F(\boldsymbol{x}))=\min & \sum_{r \in \mathscr{M}} g_{r} y_{r} & \\
\text { s.t. } & \sum_{r \in \mathscr{M}} y_{r} \leq m^{1}, & \\
& \sum_{r \in \mathscr{M}_{k}} q_{k r}=\omega_{k}, & k \in N_{S}, \\
& \sum_{k \in R_{r}} q_{k r} \leq Q_{1} y_{r}, & r \in \mathscr{M}, \\
& y_{r} \in\{0,1\}, \quad \\
& q_{k r} \geq 0, & r \in \mathscr{M},
\end{array}
$$


Problem $F(\boldsymbol{x})$ is solved to optimality with an integer programming solver. Let $(\boldsymbol{y}, \boldsymbol{q})$ be the optimal $F(\boldsymbol{x})$ solution (we assume $z(F(\boldsymbol{x}))=\infty$ if problem $F(\boldsymbol{x})$ does not admit a feasible solution). If problem $F(\boldsymbol{x})$ admits a feasible solution, then the vectors $(\boldsymbol{x}, \boldsymbol{y}, \boldsymbol{q})$ represents a feasible 2E-CVRP solution of cost $z_{U B}=$ $z(F(\boldsymbol{x}))+\sum_{k \in N_{S}} \sum_{\ell \in \mathscr{R}_{k}} c_{k \ell} x_{k \ell}$.

\subsection{An exact method for solving the 2E-CVRP}

The method for solving the 2E-CVRP is based on the following re-formulation of problem $F$.

Let $\mathscr{P}=\left\{M \subseteq \mathscr{M}:|M| Q_{1} \geq q_{t o t},|M| \leq m^{1}\right\}$. We call configuration each element $M$ of the set $\mathscr{P}$. For each configuration $M \in \mathscr{P}$, let $N_{S}(M)=\bigcup_{r \in M} R_{r}, M_{k}=M \cap \mathscr{M}_{k}$, $k \in N_{S}$, and $U(M)=\sum_{r \in M} g_{r}$. An optimal 2E-CVRP solution can be computed as

$$
z(F)=\min _{M \in \mathscr{P}}\{U(M)+z(F(M))\}
$$

where $z(F(M))$ is the optimal solution cost of the following problem $F(M)$

$$
\begin{aligned}
& (F(M)) \quad z(F(M))=\min \sum_{k \in N_{S}(M)} \sum_{\ell \in \mathscr{R}_{k}} c_{k \ell} x_{k \ell} \\
& \text { s.t. } \sum_{k \in N_{S}(M)} \sum_{\ell \in \mathscr{R}_{i k}} x_{k \ell}=1, \quad i \in N_{C} \text {, } \\
& \sum_{\ell \in \mathscr{R}_{k}} x_{k \ell} \leq m_{k}, \quad k \in N_{S}(M), \\
& \begin{array}{ll}
\sum_{k \in N_{S}(M)} \sum_{\ell \in \mathscr{R}_{k}} x_{k \ell} \leq m^{2}, & \\
\sum_{\ell \in \mathscr{R}_{k}} w_{k \ell} x_{k \ell} \leq B_{k}, & k \in N_{S}(M),
\end{array} \\
& \sum_{r \in M_{k}} q_{k r}=\sum_{\ell \in \mathscr{R}_{k}} w_{k \ell} x_{k \ell}, \quad k \in N_{S}(M), \\
& \sum_{k \in R_{r}} q_{k r} \leq Q_{1}, \quad r \in M, \\
& x_{k \ell} \in\{0,1\}, \quad k \in N_{S}(M), \ell \in \mathscr{R}_{k}, \\
& q_{k r} \geq 0, \quad k \in R_{r}, r \in M \text {. }
\end{aligned}
$$

We assume $z(F(M))=\infty$ if $F(M)$ has no feasible solution for configuration $M \in \mathscr{P}$.

Problem $F(M)$ is an extension of the multi-depot capacitated vehicle routing problem (MDCVRP) considered by Baldacci and Mingozzi [2009].

The exact method we propose (i) generates the set $\mathscr{M}$ of $1^{\text {st }}$-level routes and executes bounding procedure $D P^{1}$ to compute lower bound $L D 1$ corresponding to the optimal 
cost of problem $\overline{R F}\left(\boldsymbol{\beta}^{\mathbf{1}}, \boldsymbol{\lambda}^{\mathbf{1}}, \boldsymbol{\mu}^{\mathbf{1}}\right)$ and upper bound $U B 1$; (ii) then, generates the set $\mathscr{P}$ of configurations and limits its size by using $L D 1, U B 1$ and vectors $\left(\boldsymbol{\beta}^{\mathbf{1}}, \boldsymbol{\lambda}^{\mathbf{1}}, \boldsymbol{\mu}^{\mathbf{1}}\right)$; (iii) finally, for each configuration $M \in \mathscr{P}$, solves the associated problem $F(M)$.

The last two steps of the exact method are described in detail in the following.

\subsubsection{Generating the Set of Configurations $\mathscr{P}$}

The generation of the set $\mathscr{P}$ of configurations is based on the following propositions.

Let $L B_{R}$ be a lower bound on the $2^{\text {nd }}$-level routing cost of any optimal 2E-CVRP solution computed as $L B_{R}=\sum_{i \in N_{C}} \min _{k \in N_{S}}\left\{\beta_{i k}^{1}\right\}+\sum_{i \in N_{C}} \lambda_{i}^{1}+\sum_{k \in N_{S}} m_{k} \mu_{k}^{1}+$ $m^{2} \mu_{0}^{1}$, and let $L B W(M)$ be a lower bound on $z(F(M))$ computed as $L B W(M)=$ $\sum_{i \in N_{C}} \min _{k \in N_{S}(M)}\left\{\beta_{i k}^{1}\right\}+\sum_{i \in N_{C}} \lambda_{i}^{1}+\sum_{k \in N_{S}(M)} m_{k} \mu_{k}^{1}+m^{2} \mu_{0}^{1}$.

Proposition 1. Let $z_{U B}$ be a valid upper bound on the 2E-CVRP. A configuration $M \in \mathscr{P}$ can belong to an optimal $2 \mathrm{E}-\mathrm{CVRP}$ solution if and only if it satisfies the following conditions

$$
\left.\begin{array}{ll}
\left|R_{r} \cap R_{r^{\prime}}\right| \leq 1, r, r^{\prime} \in M, r \neq r^{\prime}, & (a) \\
\sum_{r \in M} \min \left\{Q_{1}, \sum_{k \in R_{r}} m_{k} Q_{2}\right\} \geq q_{t o t}, & (b) \\
\sum_{r \in M} \sum_{k \in R_{r}} m_{k} \geq\left\lceil q_{t o t} / Q_{2}\right\rceil, & (c) \\
U(M)<z_{U B}-L B_{R}, & (d) \\
U(M)<z_{U B}-L B W(M) . & (e)
\end{array}\right\}
$$

Condition (a) is a property of the feasible solutions of the split delivery vehicle routing problem (see Dror and Trudeau [1990]). Conditions (b) and (c) are feasibility conditions. Conditions (d) and (e) follow from the properties of any optimal 2E-CVRP solution of cost less than $z_{U B}$.

Proposition 2. For a given configuration $M \in \mathscr{P}$, let $\theta(k), k \in N_{S}(M)$, be a lower bound on the quantity that must be supplied to satellite $k$ in any feasible $F(M)$ solution by the $1^{\text {st }}$-level routes $M_{k} \subseteq M$ passing through satellite $k$. Problem $F(M)$ has no feasible solution if either $\sum_{k \in N_{S}(M)}\left\lceil\theta(k) / Q_{2}\right\rceil>m^{2}$ or $\left\lceil\theta(k) / Q_{2}\right\rceil>m_{k}$, for some satellite $k \in N_{S}$. In this case, configuration $M$ can be removed from $\mathscr{P}$. 
Lower bound $\theta(k)$ can be computed as the optimal solution cost of the following problem

$$
\begin{aligned}
& \theta(k)=\min \sum_{r \in M_{k}} q_{k r} \\
& \text { s.t. } \sum_{h \in R_{r}} q_{h r} \leq Q_{1}, \quad r \in M \text {, } \\
& \sum_{r \in M} \sum_{h \in R_{r}} q_{h r}=q_{t o t}, \\
& \sum_{r \in M_{h}} q_{h r} \geq q^{m i n}, \quad h \in N_{S}(M), \\
& \sum_{r \in M_{h}} q_{h r} \leq m_{k} Q_{2}, \quad h \in N_{S}(M), \\
& q_{h r} \geq 1, \quad h \in R_{r}, r \in M,
\end{aligned}
$$

where $q^{\text {min }}=\max \left\{\min _{i \in N_{C}}\left\{q_{i}\right\}, q_{t o t}-\left(m^{2}-1\right) Q_{2}\right\}$. We assume $\theta(k)=\infty$ if problem (6.65)-(6.70) has no feasible solution.

The set $\mathscr{P}$ is generated by pure enumeration by using Propositions 1 and 2 to eliminate any configuration $M$ that cannot lead to an optimal 2E-CVRP solution.

\subsubsection{Solving Problem $F(M)$}

Problem $F(M)$ is solved with the following three-phase method. In the first phase, bounding procedure $D P^{1}$ is used to compute lower bound $L D 1(M)$ on $z(F(M))$ by replacing $\mathscr{M}$ with $M$. In the second phase, a near-optimal dual solution of the LPrelaxation of $F(M)$ strengthened by valid inequalities, called problem $\bar{F}(M)$, is computed. In the third phase, the $\bar{F}(M)$ dual solution is used to generate the subsets $\mathscr{R}_{k}^{\prime} \subseteq \mathscr{R}_{k}, k \in N_{S}(M)$, of all $2^{\text {nd }}$-level routes of any $F(M)$ optimal solution. An $F(M)$ optimal solution is obtained by replacing, in $F(M)$, each set $\mathscr{R}_{k}$ with $\mathscr{R}_{k}^{\prime}, k \in N_{S}(M)$, and solving the resulting problem, called $F^{\prime}(M)$, with an integer programming solver.

\subsubsection{Phase 1: computing lower bound $L D 1(M)$}

We execute bounding procedure $D P^{1}$, by replacing the set $\mathscr{M}$ with $M$, to compute lower bound $L D 1(M)$ and upper bound $U B 1(M)$ on $F(M)$. If $L D 1(M)$ is greater than a known upper bound on the 2E-CVRP, Phases 2 and 3 are skipped.

\subsubsection{Phase 2: solving $\bar{F}(M)$}

Problem $\bar{F}(M)$ corresponds to the LP-relaxation of problem $F(M)$ strengthened with the following valid inequalities 
a) Capacity constraints. Let $\mathscr{S}=\left\{H: H \subseteq N_{C},|H| \geq 2\right\}$. The capacity constraints are

$$
\sum_{k \in N_{S}(M)} \sum_{\ell \in \mathscr{R}_{k}: R_{k \ell} \cap H \neq \varnothing} x_{k \ell} \geq\left\lceil\frac{\sum_{i \in H} q_{i}}{Q_{2}}\right\rceil, \quad H \in \mathscr{S} .
$$

b) Clique inequalities. Let $\mathscr{R}(M)=\bigcup_{k \in N_{S}(M)} \mathscr{R}_{k}$, and let $\mathscr{G}=(\mathscr{R}(M), \mathscr{E})$ be the conflict graph associated with the route set $\mathscr{R}(M)$, where the edge set $\mathscr{E}$ contains every edge $\left\{\ell, \ell^{\prime}\right\}, \ell, \ell^{\prime} \in \mathscr{R}(M)$, such that $\ell<\ell^{\prime}$ and $R_{\pi_{\ell} \ell} \cap R_{\pi_{\ell^{\prime}} \ell^{\prime}} \neq \varnothing$. Let $\mathcal{C}$ be the set of all cliques of graph $\mathscr{G}$. The clique inequalities are

$$
\sum_{\ell \in C} x_{\pi_{\ell} \ell} \leq 1, \quad C \in \mathscr{C}
$$

Problem $\bar{F}(M)$ is solved with a CCG procedure that starts by setting $\mathscr{S}=\varnothing, \mathscr{C}=\varnothing$. The master problem is initialized with a set of elementary routes obtained from the final set of $n g$-routes generated in Phase 1 for computing lower bound $L D 1(M)$ by removing, from each $n g$-route, the customers visited more than once. At each iteration, a set of negative reduced cost routes are generated and a set of violated inequalities (6.71) and (6.72) are added as described in Baldacci and Mingozzi [2009]. The procedure ends when no negative reduced cost routes exist and no inequalities (6.71) and (6.72) are violated and provides an $\bar{F}(M)$ dual solution of cost $z(\bar{F}(M))$.

\subsubsection{Phase 3: solving $F(M)$ to optimality}

In Phase 3, two steps are performed.

(1) Define the reduced problem $F^{\prime}(M)$ resulting from $F(M)$ by

(i) Replacing the route set $\mathscr{R}_{k}, k \in N_{S}(M)$, with the largest subset $\mathscr{R}_{k}^{\prime} \subseteq \mathscr{R}_{k}$ of routes such that $c_{k \ell}^{\prime}<z_{U B}-(U(M)+z(\bar{F}(M))), \ell \in \mathscr{R}_{k}^{\prime}, k \in N_{S}(M)$, where $c_{\ell}^{\prime}$ is the reduced cost of route $\ell \in \mathscr{R}_{k}^{\prime}$ with respect to the $\bar{F}(M)$ dual solution achieved at Phase 2 and $z_{U B}$ is the current best upper bound on the 2E-CVRP.

(ii) Adding all constraints (6.71) and (6.72) saturated by the final $\bar{F}(M)$ solution.

(2) Solve problem $F^{\prime}(M)$ with a general purpose integer programming solver.

\subsubsection{Description of the Exact Method}

The exact method we propose for solving the 2E-CVRP can be described as follows.

(1) Generate the set $\mathscr{M}$ and compute a lower bound on the 2E-CVRP. 
- Generate the set $\mathscr{M}$ of $1^{\text {st }}$-level routes by pure enumeration.

- Execute bounding procedure $D P^{1}$ to produce lower and upper bounds $L D 1$ and $U B 1$.

(2) Generate the set $\mathscr{P}$ of configurations as described in $\S 6.6 .1$.

(3) Solve the 2E-CVRP.

(a) Initialize $z(F)=U B 1, L B=U B 1, z_{U B}=U B 1, \bar{P}=\varnothing$ and $r^{\max }=0$.

(b) If $\mathscr{P}=\varnothing$, then Stop.

Let $M=\operatorname{argmin}_{M^{\prime} \in \mathscr{P}}\left\{L B W\left(M^{\prime}\right)\right\}$. Remove $M$ from $\mathscr{P}$. If $L B W(M) \geq z(F)$ then $\operatorname{Stop}(z(F)$ is the optimal 2E-CVRP solution cost).

(c) Solve problem $F(M)$.

(i) Execute Phase 1 (see $\S 6.6 .2 .1$ ) to compute lower bound $L D 1(M)$ and upper bound $U B 1(M)$ on $F(M)$. Update $z_{U B}=\min \left\{z_{U B}, U B 1(M)\right\}$, $z(F)=\min \{z(F), U B 1(M)\}$, and $L B=\min \{L B, z(F)\}$.

If $L D 1(M) \geq z(F)$, go to Step 3.b.

(ii) Execute Phase 2 (see $\S 6.6 .2 .2)$ to compute lower bound $z(\bar{F}(M))$, and update $L B=\min \{L B, \max \{L D 1(M), U(M)+z(\bar{F}(M))\}\}$.

If $U(M)+z(\bar{F}(M)) \geq z(F)$, go to Step 3.b.

If the $\bar{F}(M)$ solution of cost $z(\bar{F}(M))$ is integer, update $z(F)=U(M)+$ $z(\bar{F}(M))$ and go to Step 3.b.

(iii) Execute Phase 3 (see $\S 6.6 .2 .3$ ) to solve problem $F(M)$. Let $z(F(M)$ ) be the optimal solution cost of $F^{\prime}(M)$. Update $z(F)=\min \{z(F), U(M)+$ $z(F(M))\}, \overline{\mathscr{P}}=\overline{\mathscr{P}} \cup\{M\}$ and $r^{\max }=\max \left\{r^{\max }, \sum_{k \in N_{S}(M)}\left|\mathscr{R}_{k}^{\prime}\right|\right\}$. Go to Step 3.b.

Notice that Step 3.c-(iii) is executed for any configuration $M \in \mathscr{P}$ such that $U(M)+$ $z(\bar{F}(M))<z(F)$ and the $\bar{F}(M)$ solution is not integer. Thus, if Step 3.c-(iii) is never executed, the algorithm terminates with $\overline{\mathscr{P}}=\varnothing$, implying that $L B=z(F)$ and the optimal 2E-CVRP solution corresponds to either the initial upper bound $U B 1(M)$ computed at Step 3.c-(i) or to the integer $\bar{F}(M)$ solution achieved at Step 3.c-(ii) for some configuration $M \in \mathscr{P}$.

At the end of the exact method, $L B$ represents a valid lower bound on the $2 \mathrm{E}$-CVRP because it corresponds to $L B=\min _{M \in \mathscr{P}}\{\max \{L D 1(M), U(M)+z(\bar{F}(M))\}\}$. Value $r^{\text {max }}$ is the maximum number of $2^{\text {nd }}$-level routes generated, and set $\overline{\mathscr{P}}$ contains the configurations for which the corresponding problem $F(M)$ was solved to optimality at Step 3.c-(iii). Upper bound $z_{U B}$ is the cost of the best upper bound computed at Step 1 or at Step 3.c-(i). Finally, because we impose a limit $\Delta^{\max }$ on the maximum number of $2^{n d}$-level routes, $\bigcup_{k \in N_{S}(M)} \mathscr{R}_{k}^{\prime}$, to generate at Step 3.c-(iii), whenever such limit is reached for some configuration $M \in \overline{\mathscr{P}}$, at the end of the algorithm, the value $z(F)$ is an upper bound on the $2 \mathrm{E}-\mathrm{CVRP}$ but is not necessarily the optimal solution cost. 
TABLE 6.1: Satellite coordinates of Set 6

\begin{tabular}{|c|c|c|c|c|c|c|c|c|c|c|c|c|c|}
\hline Name & $n_{S}$ & & $k=1$ & & $k=2$ & & $k=3$ & & $k=4$ & & $k=5$ & & $k=6$ \\
\hline A-n51-4 & 4 & 7 & $(21.0,47.0)$ & 11 & $(51.0,21.0)$ & 17 & $(52.0,41.0)$ & 42 & $(10.0,17.0)$ & & & & \\
\hline A-n51-5 & 5 & 5 & $(20.0,26.0)$ & 7 & $(21.0,47.0)$ & 16 & $(36.0,16.0)$ & 23 & $(42.0,57.0)$ & 39 & $(45.0,35.0)$ & & \\
\hline A-n51-6 & 6 & 3 & $(49.0,49.0)$ & 5 & $(20.0,26.0)$ & 15 & $(12.0,42.0)$ & 16 & $(36.0,16.0)$ & 49 & $(25.0,55.0)$ & 50 & $(48.0,28.0)$ \\
\hline A-n76-4 & 4 & 30 & $(52.0,26.0)$ & 36 & $(55.0,50.0)$ & 45 & $(21.0,48.0)$ & 74 & $(27.0,24.0)$ & & & & \\
\hline A-n76-5 & 5 & 33 & $(22.0,53.0)$ & 49 & $(48.0,21.0)$ & 53 & $(54.0,38.0)$ & 54 & $(55.0,57.0)$ & 74 & $(27.0,24.0)$ & & \\
\hline A-n76-6 & 6 & 6 & $(55.0,20.0)$ & 13 & $(35.0,51.0)$ & 17 & $(21.0,36.0)$ & 53 & $(54.0,38.0)$ & 54 & $(55.0,57.0)$ & 63 & $(30.0,20.0)$ \\
\hline A-n101-4 & 4 & 8 & $(20.0,50.0)$ & 22 & $(45.0,20.0)$ & 51 & $(47.0,47.0)$ & 96 & $(25.0,24.0)$ & & & & \\
\hline A-n101-5 & 5 & 3 & $(35.0,17.0)$ & 51 & $(47.0,47.0)$ & 55 & $(57.0,29.0)$ & 83 & $(15.0,47.0)$ & 90 & $(26.0,35.0)$ & & \\
\hline A-n101-6 & 6 & 5 & $(55.0,20.0)$ & 19 & $(20.0,40.0)$ & 52 & $(49.0,58.0)$ & 62 & $(12.0,24.0)$ & 69 & $(56.0,39.0)$ & 98 & $(25.0,21.0)$ \\
\hline B-n51-4 & 4 & 7 & $(21.0,47.0)$ & 11 & $(51.0,21.0)$ & 17 & $(52.0,41.0)$ & 42 & $(10.0,17.0)$ & & & & \\
\hline B-n51-5 & 5 & 5 & $(20.0,26.0)$ & 7 & $(21.0,47.0)$ & 16 & $(36.0,16.0)$ & 23 & $(42.0,57.0)$ & 39 & $(45.0,35.0)$ & & \\
\hline B-n51-6 & 6 & 3 & $(49.0,49.0)$ & 5 & $(20.0,26.0)$ & 15 & $(12.0,42.0)$ & 16 & $(36.0,16.0)$ & 49 & $(25.0,55.0)$ & 50 & $(48.0,28.0)$ \\
\hline B-n76-4 & 4 & 30 & $(52.0,26.0)$ & 36 & $(55.0,50.0)$ & 45 & $(21.0,48.0)$ & 74 & $(27.0,24.0)$ & & & & \\
\hline B-n76-5 & 5 & 33 & $(22.0,53.0)$ & 49 & $(48.0,21.0)$ & 53 & $(54.0,38.0)$ & 54 & $(55.0,57.0)$ & 74 & $(27.0,24.0)$ & & \\
\hline B-n76-6 & 6 & 6 & $(55.0,20.0)$ & 13 & $(35.0,51.0)$ & 17 & $(21.0,36.0)$ & 53 & $(54.0,38.0)$ & 54 & $(55.0,57.0)$ & 63 & $(30.0,20.0)$ \\
\hline B-n101-4 & 4 & 8 & $(20.0,50.0)$ & 22 & $(45.0,20.0)$ & 51 & $(47.0,47.0)$ & 96 & $(25.0,24.0)$ & & & & \\
\hline B-n101-5 & 5 & 3 & $(35.0,17.0)$ & 51 & $(47.0,47.0)$ & 55 & $(57.0,29.0)$ & 83 & $(15.0,47.0)$ & 90 & $(26.0,35.0)$ & & \\
\hline B-n101-6 & 6 & 5 & $(55.0,20.0)$ & 19 & $(20.0,40.0)$ & 52 & $(49.0,58.0)$ & 62 & $(12.0,24.0)$ & 69 & $(56.0,39.0)$ & 98 & $(25.0,21.0)$ \\
\hline C-n51-4 & 4 & 22 & $(62.0,42.0)$ & 26 & $(7.0,38.0)$ & 32 & $(37.0,69.0)$ & 46 & $(39.0,10.0)$ & & & & \\
\hline C-n51-5 & 5 & 26 & $(7.0,38.0)$ & 32 & $(37.0,69.0)$ & 37 & $(63.0,69.0)$ & 40 & $(59.0,15.0)$ & 44 & $(5.0,64.0)$ & & \\
\hline C-n51-6 & 6 & 22 & $(62.0,42.0)$ & 26 & $(7.0,38.0)$ & 32 & $(37.0,69.0)$ & 34 & $(46.0,10.0)$ & 37 & $(63.0,69.0)$ & 44 & $(5.0,64.0)$ \\
\hline C-n76-4 & 4 & 11 & $(40.0,66.0)$ & 25 & $(7.0,43.0)$ & 55 & $(67.0,41.0)$ & 62 & $(36.0,6.0)$ & & & & \\
\hline C-n76-5 & 5 & 55 & $(67.0,41.0)$ & 56 & $(10.0,70.0)$ & 57 & $(6.0,25.0)$ & 61 & $(64.0,4.0)$ & 67 & $(57.0,72.0)$ & & \\
\hline C-n76-6 & 6 & 19 & $(9.0,56.0)$ & 32 & $(31.0,76.0)$ & 57 & $(6.0,25.0)$ & 58 & $(65.0,27.0)$ & 60 & $(70.0,64.0)$ & 62 & $(36.0,6.0)$ \\
\hline C-n101-4 & 4 & 30 & $(64.0,42.0)$ & 33 & $(35.0,69.0)$ & 42 & $(42.0,7.0)$ & 46 & $(6.0,38.0)$ & & & & \\
\hline C-n101-5 & 5 & 30 & $(64.0,42.0)$ & 42 & $(42.0,7.0)$ & 46 & $(6.0,38.0)$ & 65 & $(15.0,77.0)$ & 66 & $(62.0,77.0)$ & & \\
\hline C-n101-6 & 6 & 16 & $(30.0,5.0)$ & 30 & $(64.0,42.0)$ & 46 & $(6.0,38.0)$ & 65 & $(15.0,77.0)$ & 66 & $(62.0,77.0)$ & 68 & $(67.0,5.0)$ \\
\hline
\end{tabular}

\subsection{Computational results}

We report on the computational results of the exact method (hereafter BMRW) described in $\$ 6.6 .3$ and its comparison with the methods of Perboli et al. [2011] (hereafter PTV) and Jepsen et al. [2011] (hereafter JSR). BMRW was coded in Fortran 77. CPLEX 12.1 was used as the linear programming and integer programming solver. All tests were run on an IBM Intel Xeon X7350 Server (2.93 GHz - 16 GB of RAM).

We considered four sets of instances from the literature: Set 2 and 3 from by Gonzales Feliu et al. [2007a], Set 4 from Crainic et al. [2010], and Set 5 from Hemmelmayr et al. [2011]. Sets 2 to 4 are available at http://people.brunel.ac.uk/ mastjjb/ jeb/orlib/vrp2einfo.html, whereas Set 5 was kindly provided by the authors. On all instances, travel costs are computed as real Euclidean distances. The 39 instances of Sets 2 and 3 feature 21, 32 or 50 customers and 2 or 4 satellites; in Set 2, satellites are randomly spread on the plane, whereas, in Set 3, satellites are located peripherally. Set 4 consists of 54 randomly generated instances with 50 customers and 2, 3 or 5 satellites; different criteria were adopted to spread satellites and customers on the plane. Set 5 is made up of 18 instances obtained by adapting LRP benchmark 
TABLE 6.2: Computational results on Set 2 instances

\begin{tabular}{lrrrrrrrrrrr}
\hline Name & $n_{s}$ & $z(F)$ & $\% L D 1$ & $t_{L D 1}$ & $|\mathscr{P}|$ & $\% U B$ & $\% L B$ & $t_{L B}$ & $|\bar{P}|$ & $r^{m a x}$ & $t_{\text {tot }}$ \\
\hline E-n22-k4-s6-17 & 2 & 417.07 & 99.9 & 0.4 & 1 & 100.0 & 100.0 & 0.5 & 0 & 0 & 0.5 \\
E-n22-k4-s8-14 & 2 & 384.96 & 99.5 & 0.4 & 1 & 100.0 & 100.0 & 0.7 & 0 & 0 & 0.7 \\
E-n22-k4-s9-19 & 2 & 470.60 & 95.4 & 0.5 & 1 & 100.0 & 100.0 & 1.2 & 0 & 0 & 1.2 \\
E-n22-k4-s10-14 & 2 & 371.50 & 99.6 & 0.5 & 1 & 100.0 & 100.0 & 0.5 & 0 & 0 & 0.5 \\
E-n22-k4-s11-12 & 2 & 427.22 & 96.5 & 0.4 & 2 & 100.5 & 100.0 & 1.3 & 0 & 0 & 1.3 \\
E-n22-k4-s12-16 & 2 & 392.78 & 96.7 & 0.5 & 2 & 100.0 & 100.0 & 1.1 & 0 & 0 & 1.1 \\
\hline E-n33-k4-s1-9 & 2 & 730.16 & 97.9 & 25.1 & 1 & 100.0 & 100.0 & 37.6 & 0 & 0 & 37.6 \\
E-n33-k4-s2-13 & 2 & 714.63 & 97.8 & 27.8 & 2 & 100.0 & 100.0 & 34.9 & 0 & 0 & 34.9 \\
E-n33-k4-s3-17 & 2 & 707.48 & 95.0 & 28.9 & 3 & 105.8 & 100.0 & 48.1 & 0 & 0 & 48.1 \\
E-n33-k4-s4-5 & 2 & 778.74 & 94.1 & 23.1 & 4 & 100.9 & 100.0 & 72.5 & 0 & 0 & 72.5 \\
E-n33-k4-s7-25 & 2 & 756.85 & 96.8 & 27.4 & 3 & 101.0 & 100.0 & 47.1 & 0 & 0 & 47.1 \\
E-n33-k4-s14-22 & 2 & 779.05 & 98.7 & 26.0 & 3 & 100.0 & 100.0 & 31.7 & 0 & 0 & 31.7 \\
\hline E-n51-k5-s3-18 & 2 & 597.49 & 93.5 & 3.0 & 5 & 100.0 & 99.8 & 23.7 & 1 & 33,547 & 25.8 \\
E-n51-k5-s5-47 & 2 & 530.76 & 98.1 & 3.1 & 4 & 101.6 & 99.8 & 25.9 & 1 & 34,110 & 27.5 \\
E-n51-k5-s7-13 & 2 & 554.81 & 94.6 & 3.3 & 6 & 100.2 & 98.9 & 37.3 & 2 & 42,075 & 55.1 \\
E-n51-k5-s12-20 & 2 & 581.64 & 95.5 & 3.1 & 3 & 100.5 & 99.3 & 27.1 & 1 & 39,033 & 44.3 \\
E-n51-k5-s28-48 & 2 & 538.22 & 95.8 & 3.2 & 6 & 100.0 & 99.7 & 40.1 & 2 & 34,797 & 44.0 \\
E-n51-k5-s33-38 & 2 & 552.28 & 95.4 & 3.8 & 3 & 100.0 & 100.0 & 13.6 & 0 & 0 & 13.6 \\
E-n51-k5-s3-5-18-47 & 4 & 530.76 & 96.6 & 6.6 & 55 & 100.0 & 99.9 & 259.2 & 1 & 62,913 & 260.8 \\
E-n51-k5-s7-13-33-38 & 4 & 531.92 & 94.7 & 7.6 & 68 & 100.0 & 99.4 & 263.6 & 1 & 68,796 & 266.6 \\
E-n51-k5-s12-20-28-48 & 4 & 527.63 & 95.6 & 9.0 & 24 & 100.0 & 99.6 & 71.8 & 1 & 67,620 & 74.2 \\
\hline & & & & & & & & & & 0
\end{tabular}

instances; among them, we only considered the 6 instances with 5 satellites and 100 customers.

We generated another set of 27 instances (called Set 6) by starting from the CVRP instances E-n51-k5, E-n76-k10 and E-n101-k14 (available at http://branchandcut. org/VRP/data). For each CVRP instance, we generated nine 2E-CVRP instances by maintaining customer locations and demands and vehicle capacity $\left(Q_{2}\right)$ of the original CVRP instance and by setting $Q_{1}=4 Q_{2}$. The 27 instances are divided in 3 classes $(A$, $B$ and $C$ ), where, in classes $A$ and $C$, the depot is on the bottom-left-hand corner at coordinates $(1,1)$ and, in class $B$, the depot location coincides with that of the original CVRP instance. The number of satellites is 4,5 or 6 , and their locations coincide with those of some customers. In class $C$, satellites are peripheral to customers, whereas, in classes $A$ and $B$, they are positioned in the middle of the customers.

Table 6.1 reports the details of the satellite coordinates of the 27 instances of the Set 6. The table reports, for each instance, the name of the instance (Name), the number of satellites $\left(n_{S}\right)$ and the list of the satellite coordinates. For each satellite $k \in N_{S}$, the index of the corresponding customer in the original CVRP instance and the corresponding $X$ and $Y$ coordinates are reported.

In all instances, the handling costs are 0 , and satellite capacities are unlimited (i.e., $H_{k}=0$ and $W_{k}=\infty, k \in N_{S}$ ). Furthermore, in Sets 2, 3 and 5 the maximum number of vehicles per satellite is unlimited (i.e., $m_{k}=\infty, k \in N_{S}$ ). Set 4 was treated differently by Jepsen et al. [2011] who considered the given upper bounds on 
TABLE 6.3: Computational results on Set 3 instances

\begin{tabular}{lrrrrrrrrrrr}
\hline Name & $n_{s}$ & $z(F)$ & $\% L D 1$ & $t_{L D 1}$ & $|\mathscr{P}|$ & $\% U B$ & $\% L B$ & $t_{L B}$ & $|\bar{P}|$ & $r^{\text {max }}$ & $t_{\text {tot }}$ \\
\hline E-n22-k4-s13-14 & 2 & 526.15 & 96.4 & 0.4 & 4 & 100.0 & 100.0 & 2.1 & 0 & 0 & 2.1 \\
E-n22-k4-s14-19 & 2 & 498.80 & 93.2 & 0.5 & 6 & 100.0 & 100.0 & 2.4 & 0 & 0 & 2.4 \\
E-n22-k4-s13-16 & 2 & 521.09 & 94.9 & 0.4 & 4 & 100.0 & 100.0 & 2.8 & 0 & 0 & 2.8 \\
E-n22-k4-s17-19 & 2 & 512.80 & 95.5 & 0.5 & 4 & 100.0 & 100.0 & 2.6 & 0 & 0 & 2.6 \\
E-n22-k4-s13-17 & 2 & 496.38 & 96.8 & 0.5 & 1 & 100.0 & 100.0 & 1.2 & 0 & 0 & 1.2 \\
E-n22-k4-s19-21 & 2 & 520.42 & 94.9 & 0.5 & 5 & 100.0 & 100.0 & 3.8 & 0 & 0 & 3.8 \\
\hline E-n33-k4-s22-26 & 2 & 680.37 & 94.7 & 28.2 & 3 & 100.1 & 99.8 & 71.8 & 1 & 23,690 & 73.3 \\
E-n33-k4-s16-22 & 2 & 672.17 & 92.0 & 32.6 & 5 & 102.0 & 99.4 & 115.4 & 1 & 26,474 & 127.5 \\
E-n33-k4-s16-24 & 2 & 666.02 & 94.6 & 38.0 & 5 & 100.1 & 99.9 & 125.2 & 1 & 23,040 & 128.4 \\
E-n33-k4-s24-28 & 2 & 670.43 & 95.6 & 31.0 & 3 & 100.0 & 100.0 & 73.1 & 1 & 24,266 & 78.8 \\
E-n33-k4-s19-26 & 2 & 680.37 & 94.0 & 27.3 & 3 & 100.1 & 99.6 & 70.8 & 2 & 22,549 & 72.8 \\
E-n33-k4-s25-28 & 2 & 650.58 & 95.7 & 30.7 & 3 & 100.3 & 100.0 & 56.0 & 0 & 0 & 56.0 \\
\hline E-n51-k5-s13-19 & 2 & 560.73 & 95.6 & 3.3 & 5 & 100.0 & 99.6 & 43.8 & 2 & 34,800 & 48.0 \\
E-n51-k5-s13-42 & 2 & 564.45 & 97.8 & 3.6 & 1 & 100.3 & 99.1 & 18.3 & 1 & 44,083 & 50.1 \\
E-n51-k5-s13-44 & 2 & 564.45 & 96.8 & 3.2 & 3 & 101.4 & 99.0 & 29.7 & 1 & 50,444 & 73.0 \\
E-n51-k5-s40-42 & 2 & 746.31 & 91.2 & 3.6 & 5 & 102.6 & 99.0 & 34.8 & 1 & 53,016 & 107.2 \\
E-n51-k5-s41-42 & 2 & 771.56 & 97.7 & 5.7 & 2 & 100.1 & 98.9 & 36.9 & 1 & 315,861 & $2,078.6$ \\
E-n51-k5-s41-44 & 2 & 802.91 & 91.8 & 4.1 & 4 & 101.2 & 99.6 & 39.8 & 1 & 39,979 & 59.4 \\
\hline
\end{tabular}

the maximum number of vehicles per satellite, $m_{k}$, and Perboli et al. [2011] who ignored such values. To compare BMRW with both JSR and PTV, we considered two versions of Set 4, namely Set 4A and Set4B, where Set $4 \mathrm{~A}$ corresponds to the original Set 4 whereas, in Set $4 \mathrm{~B}, m_{k}$ is unbounded (i.e., $m_{k}=\infty, k \in N_{S}$ ).

Perboli et al. [2011] considered and solved to optimality 66 instances with 12 customers and 2 satellites (therein Set 1), as well. We do not report the results of BMRW on such instances because they were easily solved in a few seconds.

According to SPEC (http://www.spec.org/benchmarks.html), our machine is $10 \%$ faster than the Intel(R) Xeon X5550 $2.67 \mathrm{GHz}$ with $24 \mathrm{~GB}$ of memory and 8 cores used by JSR and twice as fast as the $3 \mathrm{GHz}$ Pentium PC with 1 GB of Ram used by PTV. A time limit of 10,000 seconds was imposed on PTV and JSR.

In testing BMRW, we used the following parameter settings. In procedure $D P^{1}$, we set $\Delta\left(N_{i}\right)=12$, and we set Maxit $1=25, \epsilon=1.0$, Maxit $2=200$, at Step 1, and Maxit $1=10, \epsilon=0.5$, Maxit $2=100$ at Step 3.c-(i). Moreover, we set $\Delta^{\max }=10^{6}$ and imposed a time limit of 5,000 seconds to solve problem $F^{\prime}(M)$.

Tables 6.2-6.7 report the results obtained by BMRW on the 6 sets of instances. The tables report the instance name, the number $n_{s}$ of satellites, the cost $z(F)$ of the best solution found, the percentage ratio $\% L D 1$ of lower bound $L D 1$ over $z(F)$ (i.e., $\% L D 1=100 L D 1 / z(F)$ ), the time $t_{L D 1}$ in seconds for computing $L D 1$, the cardinality $|\mathscr{P}|$ of the set $\mathscr{P}$ at the beginning of Step 3 , the percentage ratio $\% U B$ of upper bound $z_{U B}$ over $z(F)$, the percentage ratio $\% L B$ of lower bound $L B$ over $z(F)$, the total time $t_{L B}$ in seconds for computing $L D 1$ and $L B$, the cardinality $|\overline{\mathscr{P}}|$ of the set $\overline{\mathscr{P}}$, the value of $r^{\max }$, and finally the total computing time $t_{\text {tot }}$ in seconds. Whenever $|\overline{\mathscr{P}}|>0$, the 
difference $t_{t o t}-t_{L B}$ is the time spent by CPLEX for solving the problems $F^{\prime}(M)$, for all configurations $M \in \overline{\mathscr{P}}$.

Tables 6.8-6.10 compare BMRW with PTV and JSR. Under the headings "PTV" and "JSR", we report the percentage ratio, over $z(F)$, of the upper bound $(\% U B)$ and of the lower bound $(\% L B)$ achieved at the root node, the percentage gap (\%gap) between the best lower and upper bound computed, and the total computing time $\left(t_{t o t}\right)$ in seconds. The values in columns $z(F)$ are in bold whenever the instances were open before BMRW. The last lines of the tables reports, for each method, the number of instances solved to optimality (in columns \%gap), and, for JSR and BMRW, the average percentage deviation of the upper and lower bounds (in columns $\% U B$ and $\% L B$ ) and the average computing time (in columns $t_{t o t}$ ), computed over all instances solved by JSR, that are a subset of the instances solved by BMRW.

Tables 6.2-6.7 show that BMRW solved to optimality 166 out of 180 instances. Columns $|\mathscr{P}|$ and $|\overline{\mathscr{P}}|$ show the effectiveness of both the procedure applied at Step 2 for generating the set $\mathscr{P}$ and the procedures applied at Steps 3.c-(i) and 3.c-(ii) for computing valid lower bounds on $z(F(M))$. Notice that few problems $F(M)$ required solving by CPLEX (see columns $|\overline{\mathscr{P}}|$ ). BMRW was able to solve 25 out of 33 instances of the Sets 5 and 6 (see Tables 6.6-6.7). On the other 8 instances, BMRW could not generate all

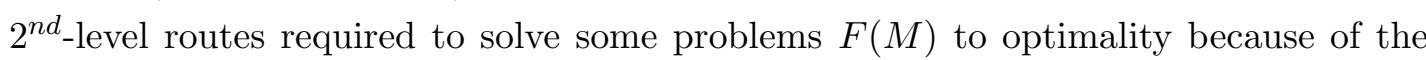
gap between the computed lower and upper bounds.

On the Sets 2 and 3 (see Tables 6.8 and 6.9), BMRW solved to optimality all 39 instances whereas PTV and JSR solved to optimality 13 and 32 instances, respectively. Of the 54 instances of the Set 4A, BMRW and JSR solved 50 and 15 of them, respectively. None of the 18 instances considered by PTV of Set $4 \mathrm{~B}$ were solved to optimality whereas 52 instances out of 54 were solved to optimality by BMRW. Tables 6.8-6.10 show that BMRW outperforms both PTV and JSR.

Finally, Tables 6.8 and 6.9 indicate that the heuristic algorithm described in $§ 6.5 .5$ provided better solutions, on average, than the heuristic algorithms of PTV and JSR.

\subsection{Conclusions}

In this chapter, we proposed a new exact method for solving the two-echelon capacitated vehicle routing problem (2E-CVRP). We described a bounding procedure that is used by the exact algorithm to decompose the 2E-CVRP into a limited set of multidepot capacitated vehicle routing problems (MDCVRP) with side constraints. The optimal 2E-CVRP solution is obtained by solving the set of MDCVRPs generated.

The proposed method was tested on 180 instances, both taken from the literature and newly generated, with up to 100 customers and 6 satellites. The new exact algorithm 
TABLE 6.4: Computational results on Set 4A instances

\begin{tabular}{|c|c|c|c|c|c|c|c|c|c|c|c|}
\hline Name & $n_{s}$ & $z(F)$ & $\% L D 1$ & $t_{L D 1}$ & $|\mathscr{P}|$ & $\% U B$ & $\% L B$ & $t_{L B}$ & $|\overline{\mathscr{P}}|$ & $r^{\max }$ & $t_{t o t}$ \\
\hline Instance50-1 & 2 & $1,569.42$ & 97.1 & 23.3 & 2 & 100.1 & 99.9 & 72.5 & 2 & 30,903 & 75.5 \\
\hline Instance50-2 & 2 & $1,438.33$ & 95.8 & 14.6 & 3 & 100.9 & 99.6 & 110.7 & 2 & 44,411 & 161.9 \\
\hline Instance50-3 & 2 & $1,570.43$ & 97.1 & 23.3 & 2 & 102.6 & 99.9 & 67.8 & 1 & 31,377 & 70.6 \\
\hline Instance 50-4 & 2 & $1,424.04$ & 96.4 & 20.2 & 2 & 101.7 & 99.4 & 59.6 & 1 & 44,211 & 101.8 \\
\hline Instance50-5 & 2 & $2,193.52$ & 98.3 & 25.1 & 5 & 100.2 & 99.6 & 286.2 & 5 & 67,491 & 663.7 \\
\hline Instance50-6 & 2 & $1,279.87$ & 95.2 & 18.5 & 2 & 100.0 & 100.0 & 42.7 & 0 & 0 & 42.7 \\
\hline Instance50-7 & 2 & $1,458.63$ & 98.0 & 29.7 & 2 & 104.7 & 99.8 & 92.7 & 2 & 30,995 & 100.4 \\
\hline Instance $50-8$ & 2 & $1,363.74$ & 95.5 & 20.8 & 3 & 100.1 & 99.5 & 199.2 & 2 & 404,659 & $2,261.9$ \\
\hline Instance 50-9 & 2 & $1,450.27$ & 98.0 & 28.5 & 2 & 104.5 & 99.9 & 82.6 & 1 & 29,683 & 84.6 \\
\hline Instance50-10 & 2 & $1,407.65$ & 92.9 & 22.9 & 2 & 100.3 & 99.6 & 71.9 & 1 & 52,513 & 112.9 \\
\hline Instance50-11 & 2 & $2,047.46$ & 99.0 & 35 & 5 & 100.8 & 99.5 & 225.5 & 5 & 88,929 & 339.1 \\
\hline Instance50-12 & 2 & $1,209.42$ & 93.1 & & 2 & 100.1 & 100.0 & 69.4 & 0 & 0 & 69.4 \\
\hline Instance50-13 & 2 & $1,481.83$ & 95.5 & & 2 & 102.4 & & 86.1 & 2 & 30,277 & 92.1 \\
\hline Instance50-14 & 2 & $1,393.61$ & 93.6 & 21.8 & 3 & 100.9 & 99.4 & 126.2 & 2 & 181,889 & $1,188.3$ \\
\hline Instance 50-15 & 2 & $1,489.94$ & 95.5 & 25.1 & 2 & 102.4 & 99.8 & 66.6 & 1 & 30,130 & 71.5 \\
\hline Instance50-16 & 2 & $1,389.17$ & 95.0 & 16.1 & 2 & 101.1 & 99.8 & 56.0 & 1 & 36,097 & 62.6 \\
\hline Instance50-17 & 2 & $2,088.49$ & 97.3 & 28.8 & 5 & 100.7 & 99.8 & 253.0 & 2 & 40,721 & 305.3 \\
\hline Instance50-18 & 2 & $1,227.61$ & 93.1 & 16.9 & 2 & 100.0 & 99.3 & 60.5 & 1 & 49,390 & 117.2 \\
\hline Instance50-19 & 3 & $1,564.66$ & 92.5 & 72.7 & 8 & 100.0 & 99.3 & 179.3 & 2 & 53,950 & 234.3 \\
\hline Instance 50-20 & 3 & $1,272.97$ & 3.7 & & 8 & 101.3 & & 59.4 & 1 & 84,784 & 140.1 \\
\hline Instanc & 3 & 1,57 & .0 & & 4 & 100.1 & & 139.8 & 2 & 58,059 & 218.9 \\
\hline Instance $50-22$ & 3 & $1,281.83$ & 95.1 & 33.9 & 8 & 101.4 & 100.0 & 76.2 & 1 & 50,177 & 79.2 \\
\hline Instance 50-23 & 3 & $1,807.35$ & 89.3 & 52.1 & 11 & 100.0 & 98.7 & 310.9 & 3 & 190,099 & $1,510.9$ \\
\hline Instance 50-24 & 3 & $1,282.68$ & 95.1 & 28.6 & 14 & 100.0 & 100.0 & 80.0 & 0 & 0 & 80.0 \\
\hline Instance 50-25 & 3 & $1,522.42$ & 91.3 & 63.0 & 8 & 102.0 & 99.2 & 221.7 & 2 & 67,095 & 335.9 \\
\hline Instance 50-26 & 3 & $1,167.46$ & 97.2 & 27.1 & 1 & 100.2 & 99.9 & 51.9 & 1 & 49,094 & 54.0 \\
\hline Instance50-27 & 3 & $1,481.57$ & 93.9 & 72.2 & 4 & 102.0 & 99.3 & 196.0 & 2 & 67,175 & 355.9 \\
\hline Instance50-28 & 3 & $1,210.44$ & 93.2 & 38.3 & 10 & 100.0 & 100.0 & 279.5 & 1 & 55,285 & 295.6 \\
\hline Instance50-29 & 3 & $1,722.04$ & 89.9 & 67.7 & 12 & 102.5 & 98.8 & 461.4 & 3 & $\Delta^{\max }$ & $9,092.9$ \\
\hline Instance 50-30 & 3 & $1,211.59$ & 93.5 & 32.8 & 13 & 100.5 & 100.0 & 243.1 & 0 & 0 & 243.1 \\
\hline Instance50-31 & 3 & $1,490.34$ & 91.8 & 65.1 & 8 & 102.2 & 98.1 & 325.9 & 4 & $\Delta^{\max }$ & $11,561.3$ \\
\hline Instance50-32 & 3 & $1,199.00$ & 94.1 & 25.9 & 7 & 100.1 & 98.7 & 262.7 & 1 & 619,322 & $4,009.4$ \\
\hline Instance50-33 & 3 & $1,508.30$ & 93.4 & 64.5 & 6 & 101.2 & 98.0 & 234.6 & 2 & $\Delta^{\max }$ & $12,922.3$ \\
\hline Instance50-34 & 3 & $1,233.92$ & 93.2 & 30.7 & 10 & 100.0 & 99.0 & 130.8 & 1 & 85,850 & 207.0 \\
\hline Instance 50-35 & 3 & $1,718.41$ & 87.6 & 63.9 & 12 & 100.1 & 98.3 & 619.9 & 5 & $\Delta^{\max }$ & $20,377.6$ \\
\hline Instance50-36 & 3 & $1,228.89$ & 93.3 & 28.6 & 14 & 100.0 & 99.3 & 121.6 & 1 & 59,745 & 154.1 \\
\hline Instance50-37 & 5 & $1,528.73$ & 94.5 & 162.7 & 116 & 100.7 & 99.5 & 778.3 & 3 & 82,225 & 807.8 \\
\hline Instance50-38 & 5 & $1,169.20$ & 93.9 & 52.5 & 134 & 100.9 & 99.0 & 429.3 & 1 & 253,662 & $1,648.2$ \\
\hline Instance50-39 & 5 & $1,520.92$ & 94.6 & 168.0 & 63 & 100.6 & 99.8 & 688.0 & 2 & 78,320 & 695.0 \\
\hline Instance50-40 & 5 & $1,199.42$ & 90.3 & 55.4 & 66 & 101.6 & 99.6 & 986.8 & 2 & 99,764 & 996.4 \\
\hline Instance50-41 & 5 & $1,667.96$ & 95.3 & 195.4 & 64 & 100.3 & 99.6 & $1,302.9$ & 4 & 79,889 & $1,344.7$ \\
\hline Instance50-42 & 5 & $1,194.54$ & 95.2 & 50.5 & 61 & 101.6 & 99.4 & 177.3 & 1 & 94,931 & 223.2 \\
\hline Instance $50-43$ & 5 & $1,439.67$ & 95.4 & 175.5 & 56 & 101.3 & 99.5 & $1,032.2$ & 3 & 87,237 & $1,095.7$ \\
\hline Instance 50-44 & 5 & $1,045.13$ & & 84.4 & 100 & 100.2 & 99.8 & 424.1 & 1 & 90,132 & 435.8 \\
\hline Instance50-45 & 5 & $1,450.96$ & & 160.9 & 34 & 101.7 & & 577.7 & 2 & 135,411 & 774.0 \\
\hline Instance50-46 & 5 & $1,088.77$ & & & 62 & 100.2 & 99.3 & $1,150.5$ & 5 & 131,810 & $1,345.4$ \\
\hline Instance $50-47$ & 5 & $1,587.29$ & 96.2 & 205.5 & 62 & 102.1 & 99.4 & $1,470.7$ & 2 & 98,905 & $1,566.3$ \\
\hline Instance $50-48$ & 5 & $1,082.20$ & 96.7 & 56.7 & 6 & 101.1 & 100.0 & 91.0 & 0 & 0 & 91.0 \\
\hline Instance50-49 & 5 & $1,434.88$ & 95.0 & 164.3 & 74 & 102.3 & 100.0 & 714.8 & 0 & 0 & 714.8 \\
\hline Instance 50-50 & 5 & $1,083.12$ & 93.2 & 55.1 & 134 & 100.5 & 99.1 & 869.1 & 1 & 239,534 & $1,337.0$ \\
\hline Instance50-51 & 5 & $1,398.05$ & 94.6 & 179.6 & 64 & 101.0 & 100.0 & 744.0 & 1 & 73,279 & 748.4 \\
\hline Instance50-52 & 5 & $1,125.67$ & 90.3 & 52.0 & 65 & 101.5 & 99.0 & $1,231.9$ & 7 & 167,299 & $1,533.7$ \\
\hline Instance50-53 & 5 & $1,567.77$ & 95.0 & 211.4 & 63 & 100.1 & 98.7 & $1,712.0$ & 2 & 268,139 & $4,223.3$ \\
\hline Instance50-54 & 5 & $1,127.61$ & 94.1 & 48.3 & 58 & 100.2 & 98.9 & 343.4 & 1 & 414,080 & $1,041.6$ \\
\hline
\end{tabular}


TABLE 6.5: Computational results on Set 4B instances

\begin{tabular}{|c|c|c|c|c|c|c|c|c|c|c|c|}
\hline Name & $n_{s}$ & $z(F)$ & $\% L D 1$ & $t_{L D 1}$ & $|\mathscr{P}|$ & $\% U B$ & $\% L B$ & $t_{L B}$ & $|\overline{\mathscr{P}}|$ & $r^{\max }$ & $t_{t o t}$ \\
\hline Instance50-1 & 2 & $1,569.42$ & 95.0 & 39.2 & 3 & 101.5 & 100.0 & 113.3 & 2 & 31,196 & 117.0 \\
\hline Instance 50-2 & 2 & $1,438.33$ & 95.8 & 14.7 & 5 & 101.0 & 99.7 & 129.8 & 2 & 42,949 & 188.5 \\
\hline Instance50-3 & 2 & $1,570.43$ & 95.1 & 38.9 & 3 & 101.5 & 100.0 & 95.1 & 1 & 31,017 & 97.8 \\
\hline Instance 50-4 & 2 & $1,424.04$ & 96.3 & 21.9 & 3 & 101.2 & 99.3 & 69.3 & 1 & 48,595 & 115.6 \\
\hline Instance $50-5$ & 2 & $2,193.52$ & 98.3 & 40.8 & 7 & 100.2 & 99.6 & 326.6 & 5 & 62,190 & 631.5 \\
\hline Instance50-6 & 2 & $1,279.87$ & 95.1 & 17.4 & 3 & 102.9 & 99.9 & 49.1 & 1 & 34,244 & 52.6 \\
\hline Instance50-7 & 2 & $1,408.57$ & 98.5 & 41.5 & 3 & 101.6 & 99.9 & 73.0 & 1 & 31,607 & 76.5 \\
\hline Instance $50-8$ & 2 & $1,360.32$ & 95.7 & 17.3 & 5 & 100.2 & 99.6 & 222.8 & 3 & 604,910 & $3,293.6$ \\
\hline Instance 50-9 & 2 & $1,403.53$ & 98.7 & 40.0 & 3 & 103.3 & 99.9 & 80.3 & 1 & 35,515 & 81.7 \\
\hline Instance 50-10 & 2 & $1,360.56$ & 96.1 & 20.8 & 3 & 100.0 & 100.0 & 46.3 & 0 & 0 & 46.3 \\
\hline Instance50-11 & 2 & $2,047.46$ & 99.0 & 50.6 & 7 & 100.8 & 99.5 & 296.8 & 6 & 88,960 & 450.8 \\
\hline Instance50-12 & 2 & $1,209.42$ & 93.0 & 22.1 & 3 & 100.0 & 99.9 & 106.9 & 1 & 48,537 & 111.9 \\
\hline Instance50-13 & 2 & $1,450.93$ & 96.1 & 44.1 & 3 & 102.3 & 100.0 & 94.1 & 0 & 0 & 94.1 \\
\hline Instance50-14 & 2 & $1,393.61$ & 93.6 & 20.6 & 5 & 101.1 & 99.4 & 147.4 & 2 & 175,756 & $1,069.7$ \\
\hline Instance50-15 & 2 & $1,466.83$ & 95.5 & 39.7 & 3 & 101.1 & 99.9 & 103.4 & 1 & 29,552 & 106.0 \\
\hline Instance50-16 & 2 & $1,387.83$ & 95.1 & 16.4 & 3 & 100.1 & 99.8 & 76.7 & 2 & 43,428 & 99.0 \\
\hline Instance 50-17 & 2 & $2,088.49$ & 97.3 & 44.3 & 7 & 100.6 & 99.8 & 306.0 & 2 & 38,365 & 358.3 \\
\hline Instance50-18 & 2 & $1,227.61$ & 93.1 & 17.0 & 3 & 100.0 & 99.2 & 69.7 & 1 & 51,842 & 127.8 \\
\hline Instance50-19 & 3 & $1,546.28$ & 93.7 & 86.3 & 16 & 101.2 & 99.2 & 262.5 & 1 & 60,815 & 293.4 \\
\hline Instance50-20 & 3 & $1,272.97$ & 93.8 & 24.3 & 9 & 101.3 & 99.0 & 59.7 & 1 & 107,430 & 170.9 \\
\hline Instance 50-21 & 3 & $1,577.82$ & 96.0 & 85.6 & 12 & 100.8 & 99.2 & 199.8 & 2 & 58,718 & 250.9 \\
\hline Instance50-22 & 3 & $1,281.83$ & 95.2 & 31.2 & 9 & 103.0 & 100.0 & 68.9 & 0 & 0 & 68.9 \\
\hline Instance50-23 & 3 & $1,652.98$ & 96.6 & 83.6 & 7 & 102.0 & 100.0 & 193.7 & 0 & 0 & 193.7 \\
\hline Instance50-24 & 3 & $1,282.68$ & 95.2 & 29.1 & 16 & 100.0 & 100.0 & 79.7 & 0 & 0 & 79.7 \\
\hline Instance50-25 & 3 & $1,408.57$ & 98.2 & 87.2 & 7 & 101.9 & 99.9 & 150.9 & 1 & 45,580 & 155.0 \\
\hline Instance50-26 & 3 & $1,167.46$ & 97.2 & 27.1 & 2 & 100.2 & 99.9 & 52.6 & 1 & 48,772 & 55.9 \\
\hline Instance50-27 & 3 & $1,444.51$ & 96.5 & 93.1 & 8 & 102.3 & 99.9 & 194.2 & 1 & 44,411 & 198.1 \\
\hline Instance50-28 & 3 & $1,210.44$ & 92.9 & 34.9 & 11 & 100.9 & 100.0 & 249.6 & 0 & 0 & 249.6 \\
\hline Instance50-29 & 3 & $1,552.66$ & 96.7 & 104.4 & 7 & 103.0 & 100.0 & 257.3 & 1 & 48,508 & 258.4 \\
\hline Instance 50-30 & 3 & $1,211.59$ & 93.2 & 38.6 & 16 & 100.5 & 99.9 & 239.9 & 1 & 61,768 & 245.9 \\
\hline Instance 50-31 & 3 & $1,440.86$ & 94.8 & 87.5 & 13 & 101.2 & 100.0 & 262.2 & 0 & 0 & 262.2 \\
\hline Instance 50-32 & 3 & $1,199.00$ & 94.1 & 31.1 & 8 & 100.1 & 98.7 & 116.9 & 1 & 579,861 & $3,812.3$ \\
\hline Instance50-33 & 3 & $1,478.86$ & 4 & 86.0 & 11 & 101.5 & 99.0 & 239.6 & 1 & 121,197 & 467.9 \\
\hline Instance50-34 & 3 & 1,23 & 93.1 & 27.6 & 11 & 101.1 & .1 & 166.6 & 1 & 131,443 & 287.7 \\
\hline Instance 50-35 & 3 & $1,570.72$ & 94.7 & 95.5 & 7 & 102.4 & 98.9 & 332.0 & 3 & 221,318 & $1,299.7$ \\
\hline Instance50-36 & 3 & $1,228.89$ & 93.1 & 26.8 & 16 & 100.0 & 99.2 & 126.2 & 1 & 72,167 & 180.1 \\
\hline Instance50-37 & 5 & $1,528.73$ & 93.7 & 206.7 & 223 & 102.0 & 97.9 & $1,520.3$ & 10 & $\Delta^{\max }$ & $14,522.3$ \\
\hline Instance50-38 & 5 & $1,163.07$ & 94.6 & 78.8 & 153 & 100.0 & 99.1 & 485.7 & 3 & 229,650 & $1,163.0$ \\
\hline Instance50-39 & 5 & $1,520.92$ & 93.1 & 212.4 & 112 & 100.3 & 98.8 & $1,097.6$ & 7 & 126,528 & $1,791.0$ \\
\hline Instance 50-40 & 5 & $1,163.04$ & 30 & 59.2 & 82 & 101.3 & 99.6 & 312.8 & 1 & 96,251 & 348.0 \\
\hline Instance50-41 & 5 & $1,652.98$ & & 266.4 & 117 & 101.0 & 99.6 & $2,229.6$ & 14 & 153,550 & $2,370.6$ \\
\hline Instance50-42 & 5 & $1,190.17$ & & & 75 & 101.9 & 99.2 & 238.4 & 2 & 132,823 & 432.4 \\
\hline Instance $50-43$ & 5 & 1,40 & 95.2 & 210.3 & 79 & 101.3 & 99.6 & $1,065.4$ & 1 & 94,023 & $1,098.4$ \\
\hline Instance 50-44 & 5 & $1,035.03$ & 96.4 & 67.0 & 93 & 100.9 & 100.0 & 382.9 & 1 & 84,919 & 387.2 \\
\hline Instance $50-45$ & 5 & $1,401.87$ & 95.3 & 187.3 & 55 & 102.7 & 99.6 & 464.7 & 1 & 82,077 & 484.7 \\
\hline Instance50-46 & 5 & $1,058.11$ & 94.7 & 83.5 & 65 & 100.4 & 100.0 & 426.9 & 0 & 0 & 426.9 \\
\hline Instance $50-47$ & 5 & $1,552.66$ & 95.8 & 260.8 & 103 & 103.0 & 100.0 & $1,220.1$ & 2 & 146,044 & $1,227.0$ \\
\hline Instance $50-48$ & 5 & $1,074.50$ & 97.3 & 60.0 & 6 & 100.5 & 99.9 & 121.4 & 1 & 74,056 & 125.5 \\
\hline Instance 50-49 & 5 & $1,434.88$ & 94.4 & 217.4 & 142 & 101.1 & 98.1 & $1,498.7$ & 8 & $\Delta^{\max }$ & $13,940.3$ \\
\hline Instance50-50 & 5 & $1,065.25$ & 94.8 & 89.2 & 126 & 100.0 & 99.9 & 500.7 & 1 & 83,400 & 508.0 \\
\hline Instance50-51 & 5 & $1,387.51$ & 93.9 & 225.0 & 92 & 102.2 & 98.9 & 845.9 & 2 & 113,910 & $1,299.1$ \\
\hline Instance 50-52 & 5 & $1,103.42$ & 92.0 & 56.0 & 81 & 100.1 & 99.4 & 788.8 & 1 & 109,254 & 846.0 \\
\hline Instance 50-53 & 5 & $1,545.73$ & 95.2 & 283.1 & 97 & 101.6 & 99.0 & $2,113.7$ & 3 & 232,398 & $2,395.8$ \\
\hline Instance50-54 & 5 & $1,113.62$ & 95.2 & 51.4 & 36 & 100.8 & 99.0 & 232.0 & 2 & 222,523 & $1,027.9$ \\
\hline
\end{tabular}


TABLE 6.6: Computational results on Set 5 instances

\begin{tabular}{lrrrrrrrrrrr}
\hline Name & $n_{s}$ & $z(F)$ & $\% L D 1$ & $t_{L D 1}$ & $|\mathscr{P}|$ & $\% U B$ & $\% L B$ & $t_{L B}$ & $|\overline{\mathscr{P}}|$ & $r^{\max }$ & $t_{\text {tot }}$ \\
\hline 2eVRP_100-5-1 & 5 & $1,564.46$ & 97.9 & 16.5 & 44 & 102.1 & 99.3 & 381.8 & 5 & 147,996 & $9,359.6$ \\
2eVRP_100-5-1b & 5 & $1,142.53$ & 93.8 & 36.5 & 60 & 100.0 & 94.6 & $1,339.2$ & 10 & $\Delta^{\max }$ & $24,028.9$ \\
2eVRP_100-5-2 & 5 & $1,016.32$ & 95.6 & 15.2 & 197 & 100.6 & 99.1 & 928.8 & 19 & 156,424 & $10,517.6$ \\
2eVRP_100-5-2b & 5 & 796.53 & 95.2 & 29.9 & 100 & 100.0 & 96.6 & $1,750.8$ & 10 & $\Delta^{\max }$ & $26,099.7$ \\
2eVRP_100-5-3 & 5 & $1,045.29$ & 97.5 & 16.3 & 50 & 100.3 & 99.2 & 262.5 & 13 & 137,379 & $2,930.2$ \\
2eVRP_100-5-3b & 5 & 833.94 & 95.7 & 39.7 & 95 & 100.0 & 97.6 & $1,475.1$ & 8 & $\Delta^{\max }$ & $32,693.8$ \\
\hline
\end{tabular}

TABle 6.7: Computational results on Set 6 instances

\begin{tabular}{|c|c|c|c|c|c|c|c|c|c|c|c|}
\hline Name & $n_{s}$ & $z(F)$ & $\% L D 1$ & $t_{L D 1}$ & $|\mathscr{P}|$ & $\% U B$ & $\% L B$ & $t_{L B}$ & $|\overline{\mathscr{P}}|$ & $r^{\max }$ & $t_{t o t}$ \\
\hline A-n51-4 & 4 & 652.00 & 94.6 & 6.7 & 16 & 100.3 & 99.7 & 108.0 & 4 & 55,040 & 119.1 \\
\hline A-n51-5 & 5 & 663.41 & 95.7 & 10.4 & 81 & 100.3 & 99.7 & 149.8 & 2 & 64,146 & 154.8 \\
\hline A-n51-6 & 6 & 662.51 & 94.9 & 15.9 & 246 & 100.6 & 100.0 & 262.8 & 0 & 0 & 263.1 \\
\hline A-n76-4 & 4 & 985.95 & 95.9 & 16.1 & 71 & 101.9 & 99.4 & 267.6 & 2 & 78,465 & 343.7 \\
\hline A-n76-5 & 5 & 979.15 & 95.8 & 27.6 & 281 & 101.8 & 99.6 & 818.9 & 4 & 91,512 & 857.2 \\
\hline A-n76-6 & 6 & 970.20 & 95.8 & 46.0 & 1,391 & 101.8 & 99.5 & $3,215.2$ & 8 & 108,558 & $3,327.4$ \\
\hline A-n101-4 & 4 & $1,194.17$ & 95.9 & 52.7 & 120 & 101.0 & 99.2 & $1,709.6$ & 11 & 461,313 & $5,971.3$ \\
\hline A-n101-5 & 5 & $1,211.38$ & 96.6 & 43.7 & 647 & 102.5 & 99.4 & $3,540.3$ & 4 & 234,285 & $4,823.3$ \\
\hline A-n101-6 & 6 & $1,158.98$ & 95.8 & 91.9 & 4,814 & 101.7 & 98.7 & $24,851.2$ & 51 & $\Delta^{\max }$ & $118,077.4$ \\
\hline B-n51-4 & 4 & 563.98 & 96.0 & 6.6 & 10 & 101.1 & 98.8 & 30.3 & 1 & 87,968 & 56.8 \\
\hline B-n51-5 & 5 & 549.23 & 94.8 & 9.7 & 64 & 101.0 & 99.0 & 105.8 & 1 & 100,364 & 130.9 \\
\hline B-n51-6 & 6 & 556.32 & 94.3 & 15.5 & 106 & 100.0 & 100.0 & 125.5 & 0 & 0 & 125.6 \\
\hline B-n76-4 & 4 & 792.73 & 94.5 & 12.4 & 23 & 102.1 & 99.3 & 257.9 & 2 & 119,142 & 333.7 \\
\hline B-n76-5 & 5 & 783.93 & 94.0 & 21.3 & 167 & 101.5 & 99.3 & 572.2 & 1 & 144,513 & 610.8 \\
\hline B-n76-6 & 6 & 774.17 & 94.4 & 32.6 & 877 & 102.2 & 99.6 & $2,023.2$ & 2 & 164,489 & $2,075.0$ \\
\hline B-n101-4 & 4 & 939.21 & 97.3 & 42.1 & 10 & 102.0 & 98.9 & 195.7 & 1 & 485,177 & $2,512.5$ \\
\hline B-n101-5 & 5 & 967.82 & 94.8 & 37.3 & 587 & 102.1 & 99.1 & $4,772.3$ & 7 & 331,423 & $7,058.8$ \\
\hline B-n101-6 & 6 & 960.29 & 96.2 & 76.9 & 456 & 103.0 & 99.1 & $1,970.4$ & 4 & 273,755 & $3,772.4$ \\
\hline C-n51-4 & 4 & 689.18 & 95.5 & 6.7 & 26 & 100.3 & 99.4 & 54.2 & 1 & 51,909 & 78.3 \\
\hline C-n51-5 & 5 & 723.12 & 93.6 & 10.3 & 64 & 100.3 & 98.9 & 81.4 & 1 & 125,120 & 431.6 \\
\hline C-n51-6 & 6 & 697.00 & 94.4 & 16.3 & 126 & 101.1 & 99.4 & 145.8 & 1 & 76,426 & 166.0 \\
\hline C-n76-4 & 4 & $1,054.89$ & 94.9 & 15.5 & 66 & 102.9 & 99.1 & 290.7 & 4 & 108,471 & 454.3 \\
\hline C-n76-5 & 5 & $1,115.32$ & 92.5 & 21.6 & 303 & 103.0 & 99.2 & $1,089.2$ & 6 & 136,054 & $1,817.0$ \\
\hline C-n76-6 & 6 & $1,064.72$ & 92.2 & 31.7 & 1,449 & 101.7 & 97.8 & $4,786.7$ & 20 & $\Delta^{\max }$ & $47,840.9$ \\
\hline C-n101-4 & 4 & $1,305.68$ & 95.0 & 49.3 & 116 & 101.7 & 98.4 & $1,392.8$ & 10 & $\Delta^{\max }$ & $29,626.4$ \\
\hline C-n101-5 & 5 & $1,309.42$ & 96.4 & 84.4 & 206 & 101.6 & 98.6 & $1,203.2$ & 2 & $\Delta^{\max }$ & $10,865.1$ \\
\hline C-n101-6 & 6 & $1,284.48$ & 96.2 & 94.6 & 1,373 & 103.3 & 98.7 & $6,804.4$ & 8 & $\Delta^{\max }$ & $27,969.4$ \\
\hline
\end{tabular}

solved to optimality 144 out of the 153 instances from literature and closed 97 of them for the first time. The comparison with the state-of-the-art exact methods from the literature show that new exact method outperforms the other exact methods in terms of size, number of problems solved to optimality, and computing time. 
TABLE 6.8: Comparison with the exact methods PTV and JSR on Set 2 instances

\begin{tabular}{|c|c|c|c|c|c|c|c|c|c|c|c|c|}
\hline \multirow[b]{2}{*}{ Name } & \multirow[b]{2}{*}{$z(F)$} & \multicolumn{2}{|c|}{$P T V$} & \multicolumn{4}{|c|}{$J S R$} & \multicolumn{5}{|c|}{$B M R W$} \\
\hline & & $\% U B$ & $\%$ gap & $\% U B$ & $\% L B$ & $\%$ gap & $t_{t o t}$ & $\% U B$ & $\% L B$ & $t_{L B}$ & $\%$ gap & $t_{\text {tot }}$ \\
\hline E-n22-k4-s6-17 & 417.07 & 100.0 & 0.0 & 100.0 & 96.7 & 0.0 & 0.2 & 100.0 & 100.0 & 0.5 & 0.0 & 0.5 \\
\hline E-n22-k4-s8-14 & 384.96 & 106.0 & 0.0 & 100.0 & 98.0 & 0.0 & 1.0 & 100.0 & 100.0 & 0.7 & 0.0 & 0.7 \\
\hline E-n22-k4-s9-19 & 470.60 & 100.0 & 0.0 & 113.1 & 90.4 & 0.0 & 12.4 & 100.0 & 100.0 & 1.2 & 0.0 & 1.2 \\
\hline E-n22-k4-s10-14 & 371.50 & 117.3 & 0.0 & 100.0 & 96.8 & 0.0 & 1.2 & 100.0 & 100.0 & 0.5 & 0.0 & 0.5 \\
\hline E-n22-k4-s11-12 & 427.22 & 100.0 & 0.0 & 104.1 & 94.7 & 0.0 & 3.2 & 100.5 & 100.0 & 1.3 & 0.0 & 1.3 \\
\hline E-n22-k4-s12-16 & 392.78 & 108.4 & 0.0 & 100.0 & 95.9 & 0.0 & 2.0 & 100.0 & 100.0 & 1.0 & 0.0 & 1.1 \\
\hline E-n33-k4-s1-9 & 730.16 & 100.9 & 0.0 & 100.0 & 87.3 & 0.0 & 49.4 & 100.0 & 100.0 & 37.6 & 0.0 & 37.6 \\
\hline E-n33-k4-s2-13 & 714.63 & 103.0 & 1.5 & 100.0 & 89.4 & 0.0 & 34.2 & 100.0 & 100.0 & 34.9 & 0.0 & 34.9 \\
\hline E-n33-k4-s3-17 & 707.48 & 104.5 & 1.7 & 113.2 & 91.0 & 0.0 & $1,126.8$ & 105.8 & 100.0 & 48.1 & 0.0 & 48.1 \\
\hline E-n33-k4-s4-5 & 778.74 & 104.9 & 1.5 & 100.0 & 87.6 & 0.0 & 54.9 & 100.9 & 100.0 & 72.5 & 0.0 & 72.5 \\
\hline E-n33-k4-s7-25 & 756.85 & 100.0 & 1.6 & 100.0 & 86.0 & 0.0 & 87.5 & 101.0 & 100.0 & 47.1 & 0.0 & 47.1 \\
\hline E-n33-k4-s14-22 & 779.05 & 100.0 & 1.6 & 105.9 & 88.0 & 0.0 & 2.4 & 100.0 & 100.0 & 31.7 & 0.0 & 31.7 \\
\hline E-n51-k5-s3-18 & 597.49 & 100.0 & 2.6 & 100.0 & 92.6 & 4.5 & - & 100.0 & 99.8 & 23.7 & 0.0 & 25.8 \\
\hline E-n51-k5-s5-47 & 530.76 & 102.3 & 1.8 & 102.4 & 97.0 & 0.0 & 13.3 & 101.6 & 99.8 & 25.9 & 0.0 & 27.5 \\
\hline E-n51-k5-s7-13 & 554.81 & 100.0 & 4.1 & 100.0 & 94.4 & 1.6 & - & 100.2 & 98.9 & 37.3 & 0.0 & 55.1 \\
\hline E-n51-k5-s12-20 & 581.64 & 100.4 & 3.7 & 104.2 & 94.2 & 0.0 & 213.6 & 100.5 & 99.3 & 27.1 & 0.0 & 44.3 \\
\hline E-n51-k5-s28-48 & 538.22 & 100.0 & 2.0 & 100.0 & 95.5 & 0.8 & - & 100.0 & 99.7 & 40.1 & 0.0 & 44.0 \\
\hline E-n51-k5-s33-38 & 552.28 & 104.7 & 0.7 & 100.0 & 95.8 & 0.0 & $2,114.0$ & 100.0 & 100.0 & 13.6 & 0.0 & 13.6 \\
\hline E-n51-k5-s3-5-18-47 & 530.76 & 102.2 & 2.8 & 103.3 & 94.4 & 0.0 & 84.0 & 100.0 & 99.9 & 259.2 & 0.0 & 260.8 \\
\hline E-n51-k5-s7-13-33-38 & 531.92 & 107.5 & 3.6 & 102.7 & 94.6 & 0.0 & $3,642.8$ & 100.0 & 99.4 & 263.6 & 0.0 & 266.6 \\
\hline E-n51-k5-s12-20-28-48 & 527.63 & 113.8 & 1.5 & 109.4 & 95.5 & 0.0 & 798.7 & 100.0 & 99.6 & 71.8 & 0.0 & 74.2 \\
\hline Avg./Solved & & 103.6 & 7 & 102.8 & 93.1 & 18 & 457.9 & 100.5 & 99.8 & & 21 & 53.6 \\
\hline
\end{tabular}

TABLE 6.9: Comparison with the exact methods PTV and JSR on Set 3 instances

\begin{tabular}{|c|c|c|c|c|c|c|c|c|c|c|c|c|}
\hline \multirow[b]{2}{*}{ Name } & \multirow[b]{2}{*}{$z(F)$} & \multicolumn{2}{|c|}{$P T V$} & \multicolumn{4}{|c|}{$J S R$} & \multicolumn{5}{|c|}{$B M R W$} \\
\hline & & $\% U B$ & $\% g a p$ & $\% U B$ & $\% L B$ & $\% g a p$ & $t_{t o t}$ & $\% U B$ & $\% L B$ & $t_{L B}$ & $\% g a p$ & $t_{\text {tot }}$ \\
\hline E-n22-k4-s13-14 & 526.15 & 100.1 & 0.0 & 102.2 & 98.2 & 0.0 & 3.2 & 100.0 & 100.0 & 2.1 & 0.0 & 2.1 \\
\hline E-n22-k4-s14-19 & 498.80 & 105.0 & 0.0 & 105.0 & 91.1 & 0.0 & 61.2 & 100.0 & 100.0 & 2.4 & 0.0 & 2.4 \\
\hline E-n22-k4-s13-16 & 521.09 & 100.0 & 0.0 & 101.0 & 98.2 & 0.0 & 2.3 & 100.0 & 100.0 & 2.8 & 0.0 & 2.8 \\
\hline E-n22-k4-s17-19 & 512.80 & 100.0 & 0.0 & 104.8 & 93.8 & 0.0 & 8.0 & 100.0 & 100.0 & 2.6 & 0.0 & 2.6 \\
\hline E-n22-k4-s13-17 & 496.38 & 100.0 & 0.0 & 100.0 & 93.4 & 0.0 & 1.1 & 100.0 & 100.0 & 1.2 & 0.0 & 1.2 \\
\hline E-n22-k4-s19-21 & 520.42 & 101.4 & 0.0 & 101.4 & 95.4 & 0.0 & 5.5 & 100.0 & 100.0 & 3.8 & 0.0 & 3.8 \\
\hline E-n33-k4-s22-26 & 680.37 & 100.0 & 4.2 & 101.5 & 91.0 & 0.0 & 6.3 & 100.1 & 99.8 & 71.8 & 0.0 & 73.3 \\
\hline E-n33-k4-s16-22 & 672.17 & 100.0 & 5.7 & 113.2 & 93.0 & 2.1 & - & 102.0 & 99.4 & 115.4 & 0.0 & 127.5 \\
\hline E-n33-k4-s16-24 & 666.02 & 100.4 & 6.0 & 100.0 & 96.4 & 0.0 & 747.4 & 100.1 & 99.9 & 125.2 & 0.0 & 128.4 \\
\hline E-n33-k4-s24-28 & 670.43 & 103.3 & 5.6 & 100.0 & 94.8 & 0.0 & 17.6 & 100.0 & 100.0 & 73.1 & 0.0 & 78.8 \\
\hline E-n33-k4-s19-26 & 680.37 & 100.0 & 4.7 & 109.2 & 89.4 & 0.0 & 26.4 & 100.1 & 99.6 & 70.8 & 0.0 & 72.8 \\
\hline E-n33-k4-s25-28 & 650.58 & 100.0 & 5.3 & 100.0 & 92.6 & 0.0 & 158.2 & 100.3 & 100.0 & 56.0 & 0.0 & 56.0 \\
\hline E-n51-k5-s13-19 & 560.73 & & & 100.0 & 95.5 & 0.0 & $1,007.9$ & 100.0 & 99.6 & 43.8 & 0.0 & 48.0 \\
\hline E-n51-k5-s13-42 & 564.45 & & & 106.1 & 96.6 & 0.0 & 208.3 & 100.3 & 99.1 & 18.3 & 0.0 & 50.1 \\
\hline E-n51-k5-s13-44 & 564.45 & & & 107.6 & 96.8 & 0.0 & 288.5 & 101.4 & 99.0 & 29.7 & 0.0 & 73.0 \\
\hline E-n51-k5-s40-42 & 746.31 & & & 100.9 & 88.9 & 7.5 & - & 102.6 & 99.0 & 34.8 & 0.0 & 107.2 \\
\hline E-n51-k5-s41-42 & 771.56 & & & 100.5 & 95.1 & 0.6 & - & 100.1 & 98.9 & 36.9 & 0.0 & $2,078.6$ \\
\hline E-n51-k5-s41-44 & 802.91 & & & 100.0 & 89.7 & 7.0 & - & 101.2 & 99.6 & 39.8 & 0.0 & 59.4 \\
\hline Avg./Solved & & 100.8 & 6 & 103.0 & 93.9 & 14 & 181.6 & 100.5 & 99.7 & & 18 & 42.5 \\
\hline
\end{tabular}


TABLE 6.10: Comparison with the exact method JSR on Set 4A instances

\begin{tabular}{|c|c|c|c|c|c|c|c|c|c|c|c|}
\hline \multirow[b]{2}{*}{ Name } & \multirow[b]{2}{*}{$n_{s}$} & \multirow[b]{2}{*}{$z(F)$} & \multicolumn{4}{|c|}{$J S R$} & \multicolumn{5}{|c|}{$B M R W$} \\
\hline & & & $\% U B$ & $\% L B$ & $\%$ gap & $t_{t o t}$ & $\% U B$ & $\% L B$ & $t_{L B}$ & $\%$ gap & $t_{t o t}$ \\
\hline Instance $50-1$ & 2 & $1,569.42$ & 112.9 & 91.2 & 1.7 & - & 100.1 & 99.9 & 72.5 & 0.0 & 75.5 \\
\hline Instance50-2 & 2 & $1,438.33$ & 100.0 & 91.5 & 0.0 & $1,146.7$ & 100.9 & 99.6 & 110.7 & 0.0 & 161.9 \\
\hline Instance50-3 & 2 & $1,570.43$ & 112.7 & 88.7 & 1.6 & - & 102.6 & 99.9 & 67.8 & 0.0 & 70.6 \\
\hline Instance50-4 & 2 & $1,424.04$ & 100.0 & 90.1 & 0.9 & - & 101.7 & 99.4 & 59.6 & 0.0 & 101.8 \\
\hline Instance 50-5 & 2 & $2,193.52$ & 100.3 & 85.6 & 0.4 & - & 100.2 & 99.6 & 286.2 & 0.0 & 663.7 \\
\hline Instance50-6 & 2 & $1,279.87$ & 100.0 & 97.2 & 0.0 & $4,463.4$ & 100.0 & 100.0 & 42.6 & 0.0 & 42.7 \\
\hline Instance50-7 & 2 & $1,458.63$ & 114.2 & 89.9 & 1.5 & - & 104.7 & 99.8 & 92.7 & 0.0 & 100.4 \\
\hline Instance50-8 & 2 & $1,363.74$ & 100.0 & 91.8 & 0.0 & $1,164.5$ & 100.1 & 99.5 & 199.2 & 0.0 & $2,261.9$ \\
\hline Instance50-9 & 2 & $1,450.27$ & 113.9 & 89.8 & 1.3 & - & 104.5 & 99.9 & 82.6 & 0.0 & 84.6 \\
\hline Instance 50-10 & 2 & $1,407.65$ & 101.0 & 98.4 & 0.0 & $3,933.1$ & 100.3 & 99.6 & 71.9 & 0.0 & 112.9 \\
\hline Instance 50-11 & 2 & $2,047.46$ & 100.9 & 89.7 & 0.6 & - & 100.8 & 99.5 & 225.5 & 0.0 & 339.1 \\
\hline Instance $50-12$ & 2 & $1,209.42$ & 100.0 & 95.7 & 0.0 & 22.3 & 100.1 & 100.0 & 69.4 & 0.0 & 69.4 \\
\hline Instance 50-13 & 2 & $1,481.83$ & 111.9 & 91.7 & 1.2 & - & 102.4 & 99.9 & 86.1 & 0.0 & 92.1 \\
\hline Instance50-14 & 2 & $1,393.61$ & 101.6 & 91.2 & 0.1 & - & 100.9 & 99.4 & 126.2 & 0.0 & $1,188.3$ \\
\hline Instance 50-15 & 2 & $1,489.94$ & 111.8 & 91.7 & 1.1 & - & 102.4 & 99.8 & 66.6 & 0.0 & 71.5 \\
\hline Instance50-16 & 2 & $1,389.17$ & 100.0 & 91.0 & 0.0 & $1,045.1$ & 101.1 & 99.8 & 56.0 & 0.0 & 62.6 \\
\hline Instance 50-17 & 2 & $2,088.49$ & 100.4 & 85.7 & 0.1 & - & 100.7 & 99.8 & 253.0 & 0.0 & 305.3 \\
\hline Instance 50-18 & 2 & $1,227.61$ & 100.0 & 96.5 & 0.0 & $8,130.1$ & 100.0 & 99.3 & 60.5 & 0.0 & 117.2 \\
\hline Instance50-19 & 3 & $1,564.66$ & 109.8 & 82.2 & 0.5 & - & 100.0 & 99.3 & 179.3 & 0.0 & 234.3 \\
\hline Instance 50-20 & 3 & $1,272.97$ & 116.6 & 93.2 & 0.6 & - & 101.3 & 99.1 & 59.4 & 0.0 & 140.1 \\
\hline Instance50-21 & 3 & $1,577.82$ & 106.8 & 88.5 & 0.4 & - & 100.1 & 99.2 & 139.8 & 0.0 & 218.9 \\
\hline Instance50-22 & 3 & $1,281.83$ & 105.1 & 86.2 & 0.0 & $8,636.7$ & 101.4 & 100.0 & 76.2 & 0.0 & 79.2 \\
\hline Instance50-23 & 3 & $1,807.35$ & 100.0 & 83.2 & 10.0 & - & 100.0 & 98.7 & 310.9 & 0.0 & $1,510.9$ \\
\hline Instance50-24 & 3 & $1,282.68$ & 101.8 & 93.9 & 0.0 & $6,559.9$ & 100.0 & 100.0 & 79.9 & 0.0 & 80.0 \\
\hline Instance50-25 & 3 & $1,522.42$ & 100.8 & 83.7 & 1.2 & - & 102.0 & 99.2 & 221.7 & 0.0 & 335.9 \\
\hline Instance50-26 & 3 & $1,167.46$ & 113.4 & 95.1 & 0.0 & 66.4 & 100.2 & 99.9 & 51.9 & 0.0 & 54.0 \\
\hline Instance50-27 & 3 & $1,481.57$ & 107.0 & 86.4 & 1.0 & - & 102.0 & 99.3 & 196.0 & 0.0 & 355.9 \\
\hline Instance 50-28 & 3 & $1,210.44$ & 104.1 & 86.7 & 0.0 & $2,046.0$ & 100.0 & 100.0 & 279.5 & 0.0 & 295.6 \\
\hline Instance50-29 & 3 & $1,722.04$ & 100.9 & 80.5 & 0.8 & - & 102.5 & 98.8 & 461.4 & 1.2 & $9,092.9$ \\
\hline Instance50-30 & 3 & $1,211.59$ & 101.8 & 92.1 & 0.0 & 17.4 & 100.5 & 100.0 & 243.1 & 0.0 & 243.1 \\
\hline Instance 50-31 & 3 & $1,490.34$ & 109.7 & 90.3 & 1.5 & - & 102.2 & 98.1 & 325.9 & 1.9 & $11,561.3$ \\
\hline Instance 50-32 & 3 & $1,199.00$ & 104.8 & 86.9 & 0.5 & - & 100.1 & 98.7 & 262.7 & 0.0 & 4,009.4 \\
\hline Instance50-33 & 3 & $1,508.30$ & 105.3 & 82.7 & 1.3 & - & 101.2 & 98.0 & 234.6 & 2.0 & $12,922.3$ \\
\hline Instance50-34 & 3 & 1,233.92 & 102.5 & 85.6 & 0.1 & - & 100.0 & 99.0 & 130.8 & 0.0 & 207.0 \\
\hline Instance50-35 & 3 & $1,718.41$ & 100.3 & 79.3 & 1.2 & - & 100.1 & 98.3 & 619.9 & 1.7 & $20,377.6$ \\
\hline Instance 50-36 & 3 & $1,228.89$ & 100.1 & 85.8 & 0.0 & $2,038.2$ & 100.0 & 99.3 & 121.6 & 0.0 & 154.1 \\
\hline Instance50-37 & 5 & $1,528.73$ & 108.7 & 82.6 & 2.9 & - & 100.7 & 99.5 & 778.3 & 0.0 & 807.8 \\
\hline Instance 50-38 & 5 & $1,169.20$ & 108.2 & 82.6 & 0.2 & - & 100.9 & 99.0 & 429.3 & 0.0 & $1,648.2$ \\
\hline Instance50-39 & 5 & $1,520.92$ & 106.4 & 84.6 & 0.4 & - & 100.6 & 99.8 & 688.0 & 0.0 & 695.0 \\
\hline Instance $50-40$ & 5 & $1,199.42$ & 101.0 & 81.5 & 2.6 & - & 101.6 & 99.6 & 986.8 & 0.0 & 996.4 \\
\hline Instance 50-41 & 5 & $1,667.96$ & 108.1 & 86.6 & 1.4 & - & 100.3 & 99.6 & $1,302.9$ & 0.0 & $1,344.7$ \\
\hline Instance 50-42 & 5 & $1,194.54$ & 112.8 & 83.3 & 1.2 & - & 101.6 & 99.4 & 177.3 & 0.0 & 223.2 \\
\hline Instance 50-43 & 5 & $1,439.67$ & 113.3 & 87.4 & 1.7 & - & 101.3 & 99.5 & $1,032.2$ & 0.0 & $1,095.7$ \\
\hline Instance 50-44 & 5 & $1,045.13$ & 109.5 & 80.4 & 0.0 & 144.0 & 100.2 & 99.8 & 424.1 & 0.0 & 435.8 \\
\hline Instance 50-45 & 5 & $1,450.96$ & 108.5 & 82.1 & 1.0 & - & 101.7 & 99.2 & 577.7 & 0.0 & 774.0 \\
\hline Instance50-46 & 5 & $1,088.77$ & 101.7 & 77.4 & 1.0 & - & 100.2 & 99.3 & $1,150.5$ & 0.0 & $1,345.4$ \\
\hline Instance 50-47 & 5 & $1,587.29$ & 109.7 & 83.5 & 1.0 & - & 102.1 & 99.4 & $1,470.7$ & 0.0 & $1,566.3$ \\
\hline Instance50-48 & 5 & $1,082.20$ & 115.8 & 86.1 & 0.0 & 133.4 & 101.1 & 100.0 & 91.0 & 0.0 & 91.0 \\
\hline Instance 50-49 & 5 & $1,434.88$ & 108.4 & 84.3 & 2.1 & - & 102.3 & 100.0 & 714.7 & 0.0 & 714.8 \\
\hline Instance 50-50 & 5 & $1,083.12$ & 105.4 & 77.9 & 1.7 & - & 100.5 & 99.1 & 869.1 & 0.0 & $1,337.0$ \\
\hline Instance50-51 & 5 & $1,398.05$ & 106.6 & 82.3 & 4.6 & - & 101.0 & 100.0 & 744.0 & 0.0 & 748.4 \\
\hline Instance 50-52 & 5 & $1,125.67$ & 100.2 & 81.0 & 1.1 & - & 101.5 & 99.0 & $1,231.9$ & 0.0 & $1,533.7$ \\
\hline Instance $50-53$ & 5 & $1,567.77$ & 109.4 & 83.8 & 1.3 & - & 100.1 & 98.7 & $1,712.0$ & 0.0 & $4,223.3$ \\
\hline Instance 50-54 & 5 & $1,127.61$ & 110.6 & 88.2 & 0.9 & - & 100.2 & 98.9 & 343.4 & 0.0 & $1,041.6$ \\
\hline Avg./Solved & & & 105.9 & 87.1 & 15 & $2,599.1$ & 101.0 & 99.4 & & 50 & 276.0 \\
\hline
\end{tabular}





\section{Bibliography}

Z. Akca, R. T. Berger, and T. K. Ralphs. A branch-and-price algorithm for combined location and routing problems under capacity restrictions. In J. W. Chinneck, B. Kristjansson, M. J. Saltzman, R. Sharda, S. Voß, and R. Sharda, editors, Operations Research and Cyber-Infrastructure, volume 47 of Operations Research/Computer Science Interfaces Series, pages 309-330. Springer US, 2009.

F. Alonso, M. J. Alvarez, and J. E. Beasley. A tabu search algorithm for the periodic vehicle routing problem with multiple vehicle trips and accessibility restrictions. Journal of the Operational Research Society, 59(7):963-976, 2008.

D. Applegate, R. E. Bixby, V. Chvátal, and W. Cook. Concorde - a code for solving traveling salesman problems. Release, 12 1999. http://www.tsp.gatech.edu/ concorde/downloads/downloads.htm.

D. L. Applegate, R. E. Bixby, V. Chvátal, and W. J. Cook. The traveling salesman problem: a computational study. Princeton Series in Applied Mathematics. Princeton University Press, Princeton, NJ, USA, 2007.

L. Art and H. Mauch. Dynamic Programming A Computational Tool. Studies in Computational Intelligence. Springer, Berlin / Heidelberg, 2007.

N. Ascheuer, M. Fischetti, and M. Grötschel. A polyhedral study of the asymmetric traveling salesman problem with time windows. Networks, 36(2):69-79, 2000.

N. Ascheuer, M. Fischetti, and M. Grötschel. Solving the asymmetric travelling salesman problem with time windows by branch-and-cut. Mathematical Programming, Series A, 90(3):475-506, 2001.

P. Augerat. Approche polyédrale du problème de tournées de véhicules. PhD thesis, Institut National Polytechnique de Grenoble, 1995.

P. J. Augerat, M. Belenguer, E. Benavent, A. Corberán, D. Naddef, and G. Rinaldi. Computational results with a branch and cut code for the capacitated vehicle routing problem. Technical Report 1RR949-M, ARTEMIS-IMAG, Grenoble, France, 1995.

N. Azi, M. Gendreau, and J.-Y. Potvin. An exact algorithm for a vehicle routing problem with time windows and multiple use of vehicles. European Journal of Operational Research, 202(3):756-763, 2010. 
E. Baker. An exact algorithm for the time-constrained traveling salesman problem. Operations Research, 31(5):938-945, 1983.

E. Balas. The asymmetric assignment problem and some new facets of the traveling salesman polytope on a directed graph. SIAM Journal on Discrete Mathematics, 2 (4):425-451, 1989.

E. Balas. Personal communication, 2000.

E. Balas and N. Christofides. A restricted Lagrangean approach to the traveling salesman problem. Mathematical Programming, Series A, 21(1):19-46, 1981.

E. Balas and N. Simonetti. Linear time dynamic-programming algorithms for new classes of restricted TSPs: a computational study. INFORMS Journal on Computing, 13(1):56-75, 2001.

E. Balas and P. Toth. Branch and bound methods. In E. Lawler, J. Lenstra, A. Rinnooy Kan, and D. Shmoys, editors, The Traveling Salesman Problem: A Guided Tour of Combinatorial Optimization, pages 361-401. Wiley, Chichester, 1985.

R. Baldacci and A. Mingozzi. A unified exact method for solving different classes of vehicle routing problems. Mathematical Programming, Series A, 120(2):347-380, 2009.

R. Baldacci, E. Hadjiconstantinou, and A. Mingozzi. An exact algorithm for the capacitated vehicle routing problem based on a two-commodity network flow formulation. Operations Research, 52(5):723-738, 2004.

R. Baldacci, L. D. Bodin, and A. Mingozzi. The multiple disposal facilities and multiple inventory locations rollon-rolloff vehicle routing problem. Computers $\& 3$ Operations Research, 33(9):2667-2702, 2006.

R. Baldacci, N. Christofides, and A. Mingozzi. An exact algorithm for the vehicle routing problem based on the set partitioning formulation with additional cuts. Mathematical Programming, Series A, 115(2):351-385, 2008.

R. Baldacci, E. Bartolini, A. Mingozzi, and R. Roberti. An exact solution framework for a broad class of vehicle routing problems. Computational Management Science, $7(3): 229-268,2010$.

R. Baldacci, A. Mingozzi, and R. Roberti. New route relaxation and pricing strategies for the vehicle routing problem. Operations Research, 59(5):1269-1283, 2011a.

R. Baldacci, A. Mingozzi, and R. Roberti. New state-space relaxation for solving the TSPTW. INFORMS Journal on Computing, 2011b.

R. Baldacci, A. Mingozzi, and R. Wolfler Calvo. An exact method for the capacitated location-routing problem. Operations Research, 59(5):1284-1296, 2011c. 
R. Baldacci, A. Mingozzi, and R. Roberti. Recent exact algorithms for solving the vehicle routing problem under capacity and time window constraints. European Journal of Operational Research, 218(1):1-6, 2012a.

R. Baldacci, A. Mingozzi, R. Roberti, and R. Wolfler Calvo. An exact algorithm for the two-echelon capacitated vehicle routing problem. Submitted for publication, 2012b.

J.-M. Belenguer, E. Benavent, C. Prins, C. Prodhon, and R. Wolfler Calvo. A branchand-cut method for the capacitated location-routing problem. Computers $\&$ Operations Research, 38(6):931-941, 2011.

R. E. Bellman. Dynamic Programming. Princeton University Press, U.S.A., 1957.

M. Bellmore and J. C. Malone. Pathology of traveling-salesman subtour-elimination algorithms. Operations Research, 19(2):278-307, 1971.

D. P. Bertsekas. Dynamic programming and optimal control, volume 1 of Athena Scientific optimization and computation series. Athena Scientific, 1995.

D. P. Bertsekas. Dynamic programming and optimal control, volume 2 of Athena Scientific optimization and computation series. Athena Scientific, 2000.

R. G. Bland and D. F. Shallcross. Large traveling salesman problems arising experiments in x-ray crystallography: A preliminary report on computation. Operations Research Letters, 8(3):125-128, 1989.

M. A. Boschetti, A. Mingozzi, and S. Ricciardelli. A dual ascent procedure for the set partitioning problem. Discrete Optimization, 5(4):735-747, 2008.

J. C. S. Brandão and A. Mercer. A tabu search algorithm for the multi-trip vehicle routing and scheduling problem. European Journal of Operational Research, 100(1): 180-191, 1997.

J. C. S. Brandão and A. Mercer. The multi-trip vehicle routing problem. Journal of the Operational Research Society, 49(8):799-805, 1998.

G. Carpaneto and P. Toth. Some new branching and bounding criteria for the asymmetric travelling salesman problem. Management Science, 26(7):736-743, 1980.

G. Carpaneto and P. Toth. Primal-dual algorithms for the assignment problem. Discrete Applied Mathematics, 18(2):137-153, 1987.

G. Carpaneto, M. Dell'Amico, and P. Toth. Exact solution of large-scale asymmetric traveling salesman problems. ACM Transactions on Mathematical Software, 21(4): 394-409, 1995.

A. Chabrier. Vehicle routing problem with elementary shortest path based column generation. Computers \& Operations Research, 33(10):2972-2990, 2006. 
I.-M. Chao. A tabu search method for the truck and trailer routing problem. Computers E Operations Research, 29(1):33-51, 2002.

N. Christofides. The traveling salesman problem: a guided tour of combinatorial optimization, chapter Vehicle Routing, pages 431-448. Wiley, Chichester, UK, 1985.

N. Christofides, A. Mingozzi, and P. Toth. The vehicle routing problem. In N. Christofides, A. Mingozzi, P. Toth, and C. Sandi, editors, Combinatorial Optimization, pages 315-338. John Wiley \& Sons, Chichester, UK, 1979.

N. Christofides, A. Mingozzi, and P. Toth. An algorithm for the time constrained travelling salesman problem. Technical Report IC OR 8125, Imperial College, 1981a.

N. Christofides, A. Mingozzi, and P. Toth. Exact algorithms for the vehicle routing problem, based on spanning tree and shortest path relaxation. Mathematical Programming, Series A, 20(1):255-280, 1981b.

N. Christofides, A. Mingozzi, and P. Toth. State-space relaxation procedures for the computation of bounds to routing problems. Networks, 11(2):145-164, 1981c.

G. Clarke and J. W. Wright. Scheduling of vehicles from a central depot to a number of delivery points. Operations Research, 12(4):568-581, 1964.

A. Claus. A new formulation for the travelling salesman problem. SIAM Journal on Algebraic and Discrete Methods, 5(1):21-25, 1984.

T. G. Crainic and G. Laporte. Fleet management and logistics. Center for Research on Transportation 25th anniversary series, 1971-1996. Kluwer, 1998.

T. G. Crainic, S. Mancini, G. Perboli, and R. Tadei. Clustering-based heuristics for the two-echelon vehicle routing problem. Technical Report CIRRELT-2008-46, CIRRELT, Montreal, Canada, November 2008.

T. G. Crainic, S. Mancini, G. Perboli, and R. Tadei. Two-echelon vehicle routing problem: a satellite location analysis. Procedia - Social and Behavioral Sciences, 2 (3):5944-5955, 2010.

T. G. Crainic, S. Mancini, G. Perboli, and R. Tadei. Multi-start heuristics for the two-echelon vehicle routing problem. In P. Merz and J.-K. Hao, editors, Evolutionary Computation in Combinatorial Optimization, volume 6622 of Lecture Notes in Computer Science, pages 179-190. Springer Berlin / Heidelberg, 2011.

R. F. da Silva and S. Urrutia. A general VNS heuristic for the traveling salesman problem with time windows. Discrete Optimization, 7(4):203-211, 2010.

C. D'Ambrosio, A. Lodi, and S. Martello. Combinatorial traveling salesman problem algorithms. In Wiley Encyclopedia of Operations Research and Management Science, volume 1, pages 738-747. Wiley, 2010. 
E. Danna and C. Pape. Branch-and-price heuristics: a case study on the vehicle routing problem with time windows. In G. Desaulniers, J. Desrosiers, and M. M. Solomon, editors, Column Generation, pages 99-129. Springer US, springer edition, 2005.

G. B. Dantzig and J. H. Ramser. The truck dispatching problem. Management Science, 6(1):80-91, 1959

G. B. Dantzig, D. R. Fulkerson, and S. M. Johnson. Solutions of a large-scale travelingsalesman problem. Operations Research, 2(4):393-410, 1954.

S. Dash, O. Günlük, A. Lodi, and A. Tramontani. A time bucket formulation for the traveling salesman problem with time windows. INFORMS Journal on Computing, 24(1):132-147, 2012.

M. S. Daskin. Network and Discrete Location: Models Algorithms and Applications. John Wiley \& Sons, New York, 1995.

G. Desaulniers, J. Desrosiers, and M. M. Solomon. Column generation. Business and Economics. Springer US, 2005.

G. Desaulniers, F. Lessard, and A. Hadjar. Tabu search, partial elementarity, and generalized k-path inequalities for the vehicle routing problem with time windows. Transportation Science, 42(3):387-404, 2008.

M. Desrochers and G. Laporte. Improvements and extensions to the Miller-TuckerZemlin subtour elimination constraints. Operations Research Letters, 10(1):27-36, 1990.

M. Desrochers, J. Desrosiers, and M. M. Solomon. A new optimization algorithm for the vehicle-routing problem with time windows. Operations Research, 40(2):342-354, 1992.

Z. Drezner. Facility location: a survey of applications and methods. Springer series in operations research. Springer, 1995.

M. Dror and P. Trudeau. Split delivery routing. Naval Research Logistics, 37:383-402, 1990.

Y. Dumas, J. Desrosiers, E. Gélinas, and M. M. Solomon. An optimal algorithm for the traveling salesman problem with time windows. Operations Research, 43(2):367-371, 1995.

J. Edmonds. Optimum branchings. Journal of Research of the National Bureau of Standards, Sec. B, 71B(4):233-240, 1967.

M. A. Efroymson and T. L. Ray. A branch-bound algorithm for plant location. Operations Research, 14(3):361-368, 1966 .

S. Eilon and N. Christofides. Distribution Management. Hafner Press, 1971. 
H. A. Eiselt and G. Laporte. A combinatorial optimization problem arising in dartboard design. Journal of the Operational Research Society, 42(2):113-118, 1991.

H. A. Eiselt and V. Marianov. Foundations of Location Analysis. International Series in Operations Research \& Management Science. Springer, 2011.

Eurostat. Panorama of Transport. Publications Office of the European Union, 2009.

Eurostat. Energy, transport and environment indicators. Pocketbooks. Publications Office of the European Union, 2011.

R. Z. Farahani and M. Hekmatfar. Facility Location: Concepts, Models, Algorithms and Case Studies. Contributions to Management Science. Physica-Verlag, 2009.

D. Feillet, P. Dejax, M. Gendreau, and C. Gueguen. An exact algorithm for the elementary shortest path problem with resource constraints: application to some vehicle routing problems. Networks, 44(3):216-229, 2004.

G. Finke, A. Claus, and E. Gunn. A two-commodity network flow approach to the traveling salesman problem. Congressus Numerantium, 41:167-178, 1984.

M. L. Fischer. Vehicle routing. In Network Routing, volume 8 of Handbook in Operations Research and Management Science, pages 1-33. North-Holland, Amsterdan, 1995.

M. Fischetti. Facets of the asymmetric traveling salesman polytope. Mathematics of Operations Research, 16(1):42-56, 1991.

M. Fischetti and E. Balas. A lifting procedure for the asymmetric traveling salesman polytope and a large new class of facets. Mathematical Programming, Series A, 58 (1-3):325-352, 1993.

M. Fischetti and P. Toth. An additive bounding procedure for combinatorial optimization problems. Operations Research, 37(2):319-328, 1989.

M. Fischetti and P. Toth. An additive bounding procedure for the asymmetric travelling salesman problem. Mathematical Programming, Series A, 53(1-3):173-197, 1992.

M. Fischetti and P. Toth. An efficient algorithm for the min-sum arborescence problem. ORSA Journal on Computing, 5:426-434, 1993.

M. Fischetti and P. Toth. A polyhedral approach to the asymmetric traveling salesman problem. Management Science, 43(11):1520-1536, 1997.

M. Fischetti, A. Lodi, S. Martello, and P. Toth. A polyhedral approach to simplified crew scheduling and vehicle scheduling problems. Management Science, 47(6):833$850,2001$. 
M. Fischetti, A. Lodi, and P. Toth. Solving real-world ATSP instances by branch-andcut. In M. Jünger, G. Reinelt, and G. Rinaldi, editors, Combinatorial Optimization - Eureka, You Shrink!, volume 2570 of Lecture Notes in Computer Science, pages 64-77. Springer Berlin / Heidelberg, Berlin, 2003.

M. Fischetti, A. Lodi, and P. Toth. Exact methods for the asymmetric traveling salesman problem. In G. Gutin, A. Punnen, D.-Z. Du, and P. M. Pardalos, editors, The Traveling Salesman Problem and Its Variations, volume 12 of Combinatorial Optimization, pages 169-205. Springer US, 2004.

B. Fleischmann. The vehicle routing problem with multiple use of vehicles. Facbereich Wirtschaftswissenschafte Universitat Hamburg, Hamburg, Germany, 1990.

F. Focacci, A. Lodi, and M. Milano. A hybrid exact algorithm for the TSPTW. INFORMS Journal on Computing, 14(4):403-417, 2002.

K. R. Fox, B. Gavish, and S. C. Graves. An n-constraint formulation of the (timedependent) traveling salesman problem. Operations Research, 28(4):1018-1021, 1980.

R. Fukasawa, H. Longo, J. Lysgaard, M. Poggi de Aragão, M. Reis, E. Uchoa, and R. F. Werneck. Robust branch-and-cut-and-price for the capacitated vehicle routing problem. Mathematical Programming, Series A, 106(3):491-511, 2006.

M. R. Garey and D. S. Johnson. Computers and intractability: a guide to the theory of NP completeness. W. H. Freeman \& Co., San Francisco, CA, 1979.

R. S. Garfinkel. On partitioning the feasible set in a branch-and-bound algorithm for the asymmetric traveling-salesman problem. Operations Research, 21(1):340-343, 1973.

R. S. Garfinkel. Minimizing wallpaper waste, part 1: A class of traveling salesman problems. Operations Research, 25(5):741-751, 1977.

B. Gavish and S. C. Graves. The travelling salesman problem and related problems. Working Paper GR-078-78, Operations Research Center, Massachusetts Institute of Technology, 1978.

M. Gendreau, A. Hertz, and G. Laporte. A tabu search heuristic for the vehicle routing problem. Management Science, 40(10):1276-1290, 1994.

M. Gendreau, A. Hertz, G. Laporte, and M. Stan. A generalized insertion heuristic for the traveling salesman problem with time windows. Operations Research, 46(3): 330-335, 1998.

M. T. Godinho, L. Gouveia, and P. Pesneau. On a time-dependent flow-based formulation and an updated classification of formulations for the travelling salesman 
problem. In Progress in Combinatorial Optimization, chapter 7, pages 223-254. Wiley, 2011.

B. Golden, S. Raghavan, and E. Wasil. The Vehicle Routing Problem: Latest Advances and New Challenges. Operations research/computer science interfaces series. Springer US, 2008.

B. L. Golden and A. A. Assad. Vehicle routing: methods and studies. Studies in management science and systems. North-Holland, 1988.

B. L. Golden and E. A. Wasil. Computerized vehicle routing in the soft drink industry. Operations Research, 35(1):6-17, 1987.

J. Gonzales Feliu, G. Perboli, R. Tadei, and D. Vigo. The two-echelon capacitated vehicle routing problem. Technical Report DEIS OR.INGCE 2007/2(R), DEIS, Bologna, Italy, 2007a.

J. Gonzales Feliu, G. Perboli, R. Tadei, and D. Vigo. The two-echelon capacitated vehicle routing problem. In Proceedings of the 22th European Conference on Operational Research, 2007b.

L. Gouveia. A result on projection for the vehicle routing problem. European Journal of Operational Research, 85(3):610-624, 1995.

L. Gouveia and P. Pesneau. On extended formulations for the precedence constrained asymmetric traveling salesman problem. Networks, 48(2):77-89, 2006.

L. Gouveia and J. M. Pires. The asymmetric travelling salesman problem and a reformulation of the Miller-Tucker-Zemlin constraints. European Journal of Operational Research, 112(1):134-146, 1999.

L. Gouveia and J. M. Pires. The asymmetric travelling salesman problem: on generalizations of disaggregated Miller-Tucker-Zemlin constraints. Discrete Applied Mathematics, 112(1-3):129-145, 2001.

L. Gouveia and S. Voß. A classification of formulations for the (time-dependent) travelling salesman problem. European Journal of Operational Research, 83(1):69-82, 1995.

I. Gribkovskaia, B. O. Gullberg, K. J. Hovden, and S. W. Wallace. Optimization model for a livestock collection problem. International Journal of Physical Distribution 8 Logistics Management, 36(2):136-152, 2006.

M. Grötschel and M.W. Padberg. Polyhedral theory. In E. Lawler, J. Lenstra, A. Rinnooy Kan, and D. Shmoys, editors, The Traveling Salesman Problem: A Guided Tour of Combinatorial Optimization, pages 251-305. Wiley, Chichester, 1985. 
G. Gutin and A. P. Punnen. The traveling salesman problem and its variations. Combinatorial Optimization. Kluwer Academic Publishers, 2002.

M. Held and R. M. Karp. The traveling-salesman problem and minimum spanning trees. Operations Research, 18(6):1138-1162, 1970.

M. Held and R. M. Karp. The traveling-salesman problem and minimum spanning trees: Part II. Mathematical Programming, Series A, 1(1):6-25, 1971.

V. C. Hemmelmayr, J.-F. Cordeau, and T. G. Crainic. An adaptive large neighborhood search heuristic for two-echelon vehicle routing problems arising in city logistics. Technical Report CIRRELT-2011-42, CIRRELT, Montreal, Canada, 2011.

Institute of Logistics and Distribution Management. The 1985 survey of distribution costs, 1985 .

S. Irnich and D. Villeneuve. The shortest-path problem with resource constraints and k-cycle elimination for $k \geq 3$. INFORMS Journal on Computing, 18(3):391-406, 2006.

M. Jepsen, B. Petersen, S. Spoorendonk, and D. Pisinger. Subset-row inequalities applied to the vehicle-routing problem with time windows. Operations Research, 56 (2):497-511, 2008 .

M. Jepsen, S. Spoorendonk, and S. Røpke. A branch-and-cut algorithm for the symmetric two-echelon capacitated vehicle routing problem. Transportation Science, 2011. Forthcoming.

R. Jonker and T. Volgenant. Transforming asymmetric into symmetric traveling salesman problems. Operations Research Letters, 2(4):161-163, 1983.

M. Jünger, G. Reinelt, and G. Rinaldi. The traveling salesman problem. In M. Ball, T. L. Magnanti, C. L. Monma, and G. Nemhauser, editors, Network Models, volume 7 of Handbooks in Operations Research and Management Science, pages 255330. North Holland, Amsterdam, 1995.

R. Karp. A patching algorithm for the nonsymmetric traveling-salesman problem. SIAM Journal on Computing, 8(4):561-573, 1979.

R. M. Karp. Reducibility among combinatorial optimization problems. In R.E. Miller and J.W. Thatcher, editors, Complexity of Computer Computations, pages 85-103. Plenum Press, New York, 1972.

N. Kohl and O. B. G. Madsen. An optimization algorithm for the vehicle routing problem with time windows based on Lagrangian relaxation. Operations Research, 45(3):395-406, 1997. 
N. Kohl, J. Desrosiers, O. B. G. Madsen, M. M. Solomon, and F. Soumis. 2-path cuts for the vehicle routing problem with time windows. Transportation Science, 33(1): 101-116, 1999.

T. C. Koopmans and M. Beckmann. Assignment problems and the location of economic activities. Econometrica, 25(1):53-76, 1957.

A. A. Kuehn and M. J. Hamburger. A heuristic program for locating warehouses. Management Science, 9(4):643-666, 1963.

B. J. LaLonde and P. H. Zinszer. Customer service: meaning and measurement. National Council of Physical Distribution Management, Chicago, 1976.

A. Langevin. Planification des tournées de véhicules. PhD thesis, École Polytechnique de Montréal, 1988.

A. Langevin, F. Soumis, and J. Desrosiers. Classification of travelling salesman problem formulations. Operations Research Letters, 9(2):127-132, 1990.

A. Langevin, M. Desrochers, J. Desrosiers, S. Gélinas, and F. Soumis. A twocommodity flow formulation for the traveling salesman and the makespan problems with time windows. Networks, 23(7):631-640, 1993.

G. Laporte, Y. Nobert, and D. Arpin. An exact algorithm for solving a capacitated location-routing problem. Annals of Operations Research, 6(9):291-310, 1986.

E. L. Lawler. Combinatorial Optimization: Networks and Matroids, volume 40. Holt, Rinehart and Winston, 1976.

E. L. Lawler, J. K. Lenstra, A. H. G. Rinnooy Kan, and D. B. Shmoys. The traveling salesman problem: a guided tour of combinatorial optimization. Wiley, Chichester, UK, 1985.

J. K. Lenstra and A. H. G. Rinnooy Kan. Some simple applications of the travelling salesman problem. Operational Research Quarterly, 26(4):717-733, 1975.

J.-Q. Li. A computational study of bi-directional dynamic programming for the traveling salesman problem with time windows. Working paper, 2009.

J. D. C. Little, K. G. Murty, D. W. Sweeney, and C. Karel. An algorithm for the traveling salesman problem. Operations Research, 11(6):972-989, 1963.

L. López Ibáñez and C. Blum. Beam-ACO for the travelling salesman problem with time windows. Computers \& Operations Research, 37(9):1570-1583, 2010.

R. J. Loulou. On commodity flow formulations for the TSP. Working paper, McGill University, Montréal, 1988. 
R. F. Love, J. G. Morris, and G. O. Wesolowsky. Facilities location: models 63 methods. Publications in operations research series. North-Holland, 1988.

J. Lysgaard, A. N. Letchford, and R. W. Eglese. A new branch-and-cut algorithm for the capacitated vehicle routing problem. Mathematical Programming, Series A, 100 (2):423-445, 2004.

A. S. Manne. Plant location under economies-of-scale-decentralization and computation. Management Science, 11(2):213-235, 1964.

K. Menger. Das botenproblem. Ergebnisse Eines Mathematischen Kolloquiums, 2: 11-12, 1932.

D. Mester and O. Bräysy. Active guided evolution strategies for large-scale vehicle routing problems with time windows. Computers $\&$ Operations Research, 32(6): 1593-1614, 2005.

C. E. Miller, A. W. Tucker, and R. A. Zemlin. Integer programming formulation of traveling salesman problems. Journal of the Association for Computing Machinery, $7(4): 326-329,1960$.

D. L. Miller and J. F. Pekny. Results from a parallel branch and bound algorithm for the asymmetric traveling salesman problem. Operations Research Letters, 8(3): 129-135, 1989.

A. Mingozzi, N. Christofides, and E. Hadjiconstantinou. An exact algorithm for the vehicle routing problem based on the set partitioning formulation. Technical report, University of Bologna, Bologna, Italy, 1994.

A. Mingozzi, L. Bianco, and S. Ricciardelli. Dynamic programming strategies for the traveling salesman problem with time window and precedence constraints. Operations Research, 45(3):365-377, 1997.

A. Mingozzi, R. Roberti, and P. Toth. An exact algorithm for the multi-trip vehicle routing problem. INFORMS Journal on Computing, 2012.

P. B. Mirchandani and R. L. Francis. Discrete location theory. Interscience series in discrete mathematics and optimization. Wiley, 1990.

V.-P. Nguyen, C. Prins, and C. Prodhon. A multi-start evolutionary local search for the two-echelon location routing problem. In M. Blesa, C. Blum, G. Raidl, A. Roli, and M. Sampels, editors, Hybrid Metaheuristics, volume 6373 of Lecture Notes in Computer Science, pages 88-102. Springer Berlin / Heidelberg, Berlin, Heidelberg, 2010.

J. W. Ohlmann and B. W. Thomas. A compressed-annealing heuristic for the traveling salesman problem with time windows. INFORMS Journal on Computing, 19(1):8090, 2007 . 
A. Olivera. Memorias adaptativas para el problema de ruteo de vehículos con múltiples viajes. PhD thesis, Instituto de Computación - Facultad de Ingeniería - Universidad de la República - Montevideo, Uruguay, 2005.

A. Olivera and O. Viera. Adaptive memory programming for the vehicle routing problem with multiple trips. Computers \&J Operations Research, 34(1):28-47, 2007.

T. Öncan, I. Kuban Altinel, and G. Laporte. A comparative analysis of several asymmetric traveling salesman problem formulations. Computers $\& 5$ Operations Research, 36(3):637-654, 2009.

M. Padberg and T. Sung. An analytical comparison of different formulations of the travelling salesman problem. Mathematical Programming, Series A, 52(1-3):315-357, 1991.

M. W. Padberg and G. Rinaldi. An efficient algorithm for the minimum capacity cut problem. Mathematical Programming, Series A, 47(1-3):19-36, 1990a.

M. W. Padberg and G. Rinaldi. Facet identification for the symmetric traveling salesman polytope. Mathematical Programming, Series A, 47(1-3):219-257, 1990b.

J. F. Pekny and D. L. Miller. A parallel branch and bound algorithm for solving large asymmetric traveling salesman problems. Mathematical Programming, Series A, 55 (1-3):17-33, 1992.

G. Perboli, R. Tadei, and F. Masoero. New families of valid inequalities for the twoechelon vehicle routing problem. Electronic Notes in Discrete Mathematics, 36(2): 639-646, 2010.

G. Perboli, R. Tadei, and D. Vigo. The two-echelon capacitated vehicle routing problem: models and math-based heuristics. Transportation Science, 45(3):364-380, 2011.

G. Pesant, M. Gendreau, J.-Y. Potvin, and J.-M. Rousseau. An exact constraint logic programming algorithm for the traveling salesman problem with time windows. Transportation Science, 32(1):12-29, 1998.

R. J. Petch and S. Salhi. A multi-phase constructive heuristic for the vehicle routing problem with multiple trips. Discrete Applied Mathematics, 133(1-3):69-92, 2004.

D. Pisinger and S. Ropke. A general heuristic for vehicle routing problems. Computers E Operations Research, 34(8):2403-2435, 2007.

J.-Y. Potvin and S. Bengio. The vehicle routing problem with time windows part II: Genetic search. INFORMS Journal on Computing, 8(2):165-172, 1996.

G. Reinelt. TSPLIB - a traveling salesman problem library. ORSA Journal on Computing, 3(4):376-384, 1991. 
G. Reinelt. The traveling salesman problem: computational solutions for TSP applications. Lecture Notes in Computer Science. Springer - Verlag, Berlin, 1994.

G. Righini and M. Salani. Symmetry helps: Bounded bi-directional dynamic programming for the elementary shortest path problem with resource constraints. Discrete Optimization, 3(3):255-273, 2006.

G. Righini and M. Salani. New dynamic programming algorithms for the resource constrained elementary shortest path problem. Networks, 51(3):155-170, 2008.

R. Roberti and P. Toth. Models and algorithms for the asymmetric traveling salesman problem: an experimental comparison. Submitted for publication, 2012.

J. B. Robinson. On the hamiltonian game (a traveling salesman problem). Technical Report RM-303, RAND Research Memorandum, 1949.

Y. Rochat and É. D. Taillard. Probabilistic intensification and diversification in local search for vehicle routing. Journal of Heuristics, 1(1):147-167, 1995.

S. Ropke. Heuristic and exact algorithms for vehicle routing problems. PhD thesis, Computer Science Department, University of Copenhagen (DIKU), Copenhagen, 2005.

S. Ropke. Personal communication, 2010. January 29.

S. Salhi and R. J. Petch. A GA based heuristic for the vehicle routing problem with multiple trips. Journal of Mathematical Modelling and Algorithms, 6(4):591-613, 2007.

S. C. Sarin, H. D. Sherali, and A. Bhootra. New tighter polynomial length formulations for the asymmetric travelling salesman problem with and without precedence constraints. Operations Research Letters, 33(1):62-70, 2005.

M. W. P. Savelsbergh. Local search in routing problems with time windows. Annals of Operations Research, 4(1):285-305, 1985.

H. D. Sherali and P. J. Driscoll. On tightening the relaxations of Miller-Tucker-Zemlin formulations for asymmetric traveling salesman problems. Operations Research, 50 (4):656-669, 2002

H. D. Sherali, S. C. Sarin, and P.-F. Tsai. A class of lifted path and flow-based formulations for the asymmetric travelling salesman problem with and without precedence constraints. Discrete Optimization, 3(1):20-32, 2006.

D. Simchi-Levi, X. Chen, and J. Bramel. The Logic of Logistics: Theory, Algorithms, and Applications for Logistics and Supply Chain Management. Springer Series in Operations Research and Financial Engineering. Springer New York, 2005. 
T. H. C. Smith, V. Srinivasan, and G. L. Thompson. Computational performance of three subtour elimination algorithms for solving asymmetric traveling salesman problems. In B. H. Korte P. L. Hammer, E. L. Johnson and G. L. Nemhauser, editors, Studies in Integer Programming, volume 1 of Annals of Discrete Mathematics, pages 495-506. Elsevier, 1977.

M. M. Solomon. Algorithms for the vehicle routing and scheduling problem with time window constraints. Operations Research, 35(2):254-265, 1987.

É. D. Taillard. Personal communication, 2009. June 16.

É. D. Taillard, G. Laporte, and M. Gendreau. Vehicle routeing with multiple use of vehicles. Journal of the Operational Research Society, 47(8):1065-1070, 1996.

K. C. Tan, Y. H. Chew, and L. H. Lee. A hybrid multi-objective evolutionary algorithm for solving truck and trailer vehicle routing problems. European Journal of Operational Research, 172(3):855-885, 2006.

E. Taniguchi. City logistics: network modelling and intelligent transport systems. Pergamon, 2001.

E. Taniguchi and R. G. Thompson. Innovations in city logistics. Nova Science Publishers, 2008.

R. E. Tarjan. Finding optimum branchings. Networks, 7(1):25-35, 1977.

P. Toth and D. Vigo. The vehicle routing problem. SIAM monographs on discrete mathematics and applications. Society for Industrial and Applied Mathematics, 2002.

R. Wolfler Calvo. A new heuristic for the traveling salesman problem with time windows. Transportation Science, 34(1):113-124, 2000.

R. T. Wong. Integer programming formulations of the traveling salesman problem. In Proceedings of the IEEE international conference of circuits and computers, 1980. 